\title{
Enhanced separation in ambient mass spectrometry imaging
}

Citation for published version (APA):

Lamont - de Vries, L. (2021). Enhanced separation in ambient mass spectrometry imaging: towards quantification of pharmaceuticals. [Doctoral Thesis, Maastricht University]. Ridderprint BV. https://doi.org/10.26481/dis.20210527II

Document status and date:

Published: 01/01/2021

DOI:

10.26481/dis.20210527II

Document Version:

Publisher's PDF, also known as Version of record

\section{Please check the document version of this publication:}

- A submitted manuscript is the version of the article upon submission and before peer-review. There can be important differences between the submitted version and the official published version of record.

People interested in the research are advised to contact the author for the final version of the publication, or visit the DOI to the publisher's website.

- The final author version and the galley proof are versions of the publication after peer review.

- The final published version features the final layout of the paper including the volume, issue and page numbers.

Link to publication

\footnotetext{
General rights rights.

- You may freely distribute the URL identifying the publication in the public portal. please follow below link for the End User Agreement:

www.umlib.nl/taverne-license

Take down policy

If you believe that this document breaches copyright please contact us at:

repository@maastrichtuniversity.nl

providing details and we will investigate your claim.
}

Copyright and moral rights for the publications made accessible in the public portal are retained by the authors and/or other copyright owners and it is a condition of accessing publications that users recognise and abide by the legal requirements associated with these

- Users may download and print one copy of any publication from the public portal for the purpose of private study or research.

- You may not further distribute the material or use it for any profit-making activity or commercial gain

If the publication is distributed under the terms of Article $25 \mathrm{fa}$ of the Dutch Copyright Act, indicated by the "Taverne" license above, 
2

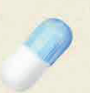

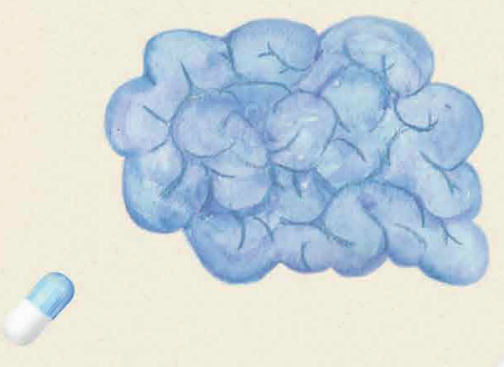

$?$

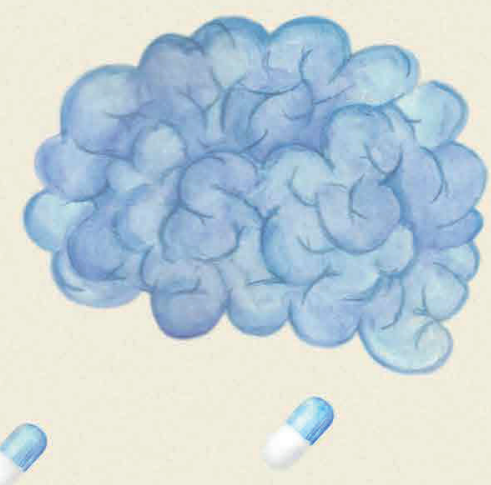

ENHANCED SEPARATION IN AMBIENT

9

\. MASS SPECTROMETRY IMAGING

TOWARDS QUANTIFICATION OF PHARMACEUTICALS

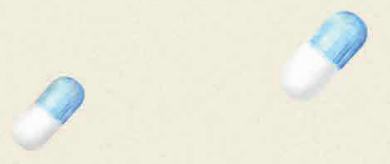

?

?

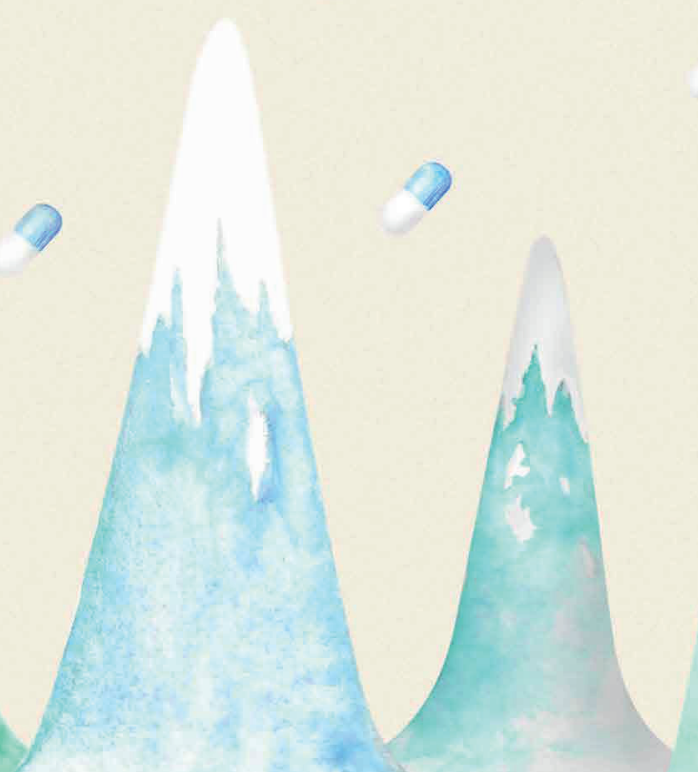

9

9

2 

Enhanced Separation in Ambient Mass Spectrometry Imaging Towards Quantification of Pharmaceuticals 
ISBN: 978-94-6416-266-0

The work described in this thesis was performed at the Maastricht MultiModal Molecular Imaging institute at Maastricht University, Maastricht, The Netherlands.

This work was supported by the Dutch Province of Limburg as part of the LINK program and the Integrated Technology Strategy (ITS) initiative program of Janssen Pharmaceutica NV.

Copyright @ Lieke Lamont-de Vries, Maastricht, 2020.

Cover design: Romina Alvarez

Printed by: Ridderprint, www.ridderprint.nl 


\title{
Enhanced Separation in Ambient Mass Spectrometry Imaging
}

\author{
Towards Quantification of Pharmaceuticals
}

\author{
PROEFSCHRIFT \\ ter verkrijging van de graad van doctor aan de Universiteit Maastricht, \\ op gezag van de Rector Magnificus, Prof.dr. Rianne M. Letschert \\ volgens het besluit van het College van Decanen, \\ in het openbaar te verdedigen \\ op donderdag 28 januari 2021 om 10.00 uur
}

door

Lieke Lamont-de Vries

Geboren op 28 september 1988 te Doetinchem 


\section{Promotor}

Prof. dr. R. M. A. Heeren

\section{Copromotors}

Dr. R. J. Vreeken

Dr. T. Porta Siegel

\section{Beoordelingscommissie}

Prof. dr. T. M. Hackeng (voorzitter)

Dr. F. Cuyckens (Janssen Pharmaceutica)

Prof. dr. P. Habibovic

Prof. dr. T. Hankemeier (Universiteit Leiden)

Prof. dr. F. J. van Schooten

This research was part of the M4I research program and received financial support from the Dutch Province of Limburg under the LINK program and the Integrated Technology Strategy (ITS) initiative program of Janssen Pharmaceutica NV. 




\section{Table of content}

Chapter 1

General Introduction

Chapter 2

High Resolution LESA- $\mu$ LC-IMS-MSE

\section{Chapter 3}

61

High Resolution IMS-MS for Isomeric Prostanoid Separation

Chapter 4

83

Targeted Drug and Metabolite Imaging

Chapter 5

97

MRM based Quantitative Mass Spectrometry Imaging

Chapter 6

119

Valorization

Chapter 7

125

Summary and Outlook

General discussion

Summary

Samenvatting

Chapter 8

Appendices

References

List of abbreviations

List of publications

Acknowledgements

Curriculum vitae 


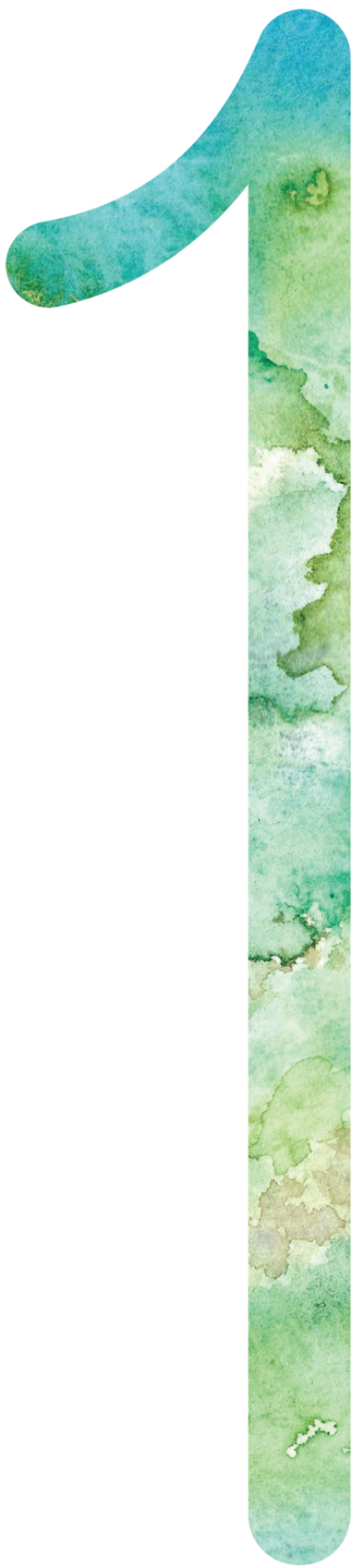


General Introduction 



\subsection{Introduction}

Analytical chemistry allows us to study and understand biological systems and diseases by exploring the chemistry and behavior of endogenous and exogenous molecules in a system. Analytical chemistry is defined by IUPAC as a scientific discipline that develops and applies methods, instruments, and strategies to obtain information on the composition and nature of matter in space and time ${ }^{1}$. In other words, disease-specific molecules can be revealed, identified, and quantified in a complex system by applying the right analytical tools. Clinical validation is required to prove the analytical performance of a quantitative method prior to a routine application of analytical methods. Validation parameters that are evaluated and established are sensitivity (expressed as the concentration measured at limit of detection), precision (expressed as standard deviation), accuracy (expressed as percentage-wise error from the exact value), and linearity (expressed as correlation coefficient)2. An acceptable outcome of a validation study, e.g. meeting predefined validation criteria, can be achieved by sufficient instrumental separation power, which is expressed by peak resolution and resolving power between two identical peaks.

Mass spectrometry (MS) is a technology that is used for chemical identification, localization, and quantification of molecules ${ }^{3}$. MS has been, often in combination with chromatography, revolutionizing the field of analytical chemistry. This chapter introduces the fundamentals of mass spectrometry, mass spectrometry imaging (MSI) and its application of small molecules. This thesis investigates the hurdles in quantitative MSI, as briefly outlined at the end of this introduction.

\subsection{Mass Spectrometry}

MS is a unique weighing scale which can measure a variety of molecules, from large intact biomolecules to single atoms and isotopes ${ }^{4}$. Since the first mass spectrum was recorded by Sir J. J. Thomson in 1912, developments in the field of MS have led to an analytical tool that is currently one of the most sensitive and fastest analytical tools available. A mass spectrometer consists of three main building blocks: an ionization source, one or more mass analyzers, and an ion detector. In the ionization source, molecules are converted into gas-phase ions. A mass analyzer separates the ions based on their mass-to-charge $(\mathrm{m} / \mathrm{z})$ ratio. If multiple mass analyzers are present in the mass spectrometer, a fragmentation cell is present between the mass analyzers to fragment selected ions from the previous mass analyzer and separate the obtained fragments in the next mass analyzer. The ion detector detects ions from the last mass analyzer, measures their abundance, and converts the ions into electrical signals ${ }^{5}$. 
In mass spectrometry, the mass of a molecule is described in dalton (Da) and defined by the sum of the mass of the predominant isotope of all atoms present in the molecule (e.g. the exact mass/theoretical mass). When this value is rounded to its integer value, it is referred to as the nominal mass of the molecule. A mass spectrum shows multiple $\mathrm{m} / \mathrm{z}$ peaks derived from one ion. The highest abundant peak (e.g. the monoisotopic peak) refers to the ion directly related to the molecular weight, either being a radical cation, a (de)pronated molecule, an adduct - or fragment-ion, all with the predominant isotopes and is followed by $\mathrm{m} / \mathrm{z}$ peaks that refer to heavier isotopes of this molecule which differ one Da from each other. Since a mass spectrum shows $\mathrm{m} / \mathrm{z}$ ratios, the difference between the monoisotopic peak and heavier isotopic peaks can reveal the amount of charges present in the ion.

\subsubsection{Ionization}

In the ionization process, a neutral molecule either loses or retrieves an electron which results in a positive or negative charged version of the molecule, called an ion. A variety of ionization techniques are available for the analysis of different classes of molecules contained in different sample types. For the right choice of ionization, three factors play an important role: i) the sample nature, ii) the molecular structure of the analyte, and iii) the transfer of internal energy during the ionization process can cause extensive fragmentation of the molecules. In biomolecular MS, two different ionization techniques are most frequently used: electrospray ionization (ESI) and matrix-assisted laser desorption ionization (MALDI).

\section{Matrix-assisted laser desorption ionization}

Matrix-assisted laser desorption ionization (MALDI) was introduced by Karas and Hillenkamp in 1988 and allows the production of intact gas-phase ions ${ }^{6}$. MALDI produces those ions in a two-step process. First, the analytes are dissolved in a solution that contains small organic molecules, which is called the matrix. This solution is deposited onto a target plate and all solvent is air-dried. This results in analyte-doped matrix crystals in which all analytes are surrounded by matrix crystals and completely isolated from each other. In the second step, the target plate is placed in the source under vacuum conditions. A laser is used to irradiate the matrix crystals which are cocrystalized with the analyte molecules. The excitation of the matrix molecules by the laser causes accumulation of large amounts of energy and results in local sublimation of the matrix crystals, expansion of the matrix into the gas plume and charge transfer of the matrix to the analyte. Exact details of this mechanism are still not fully understood. The most accepted explanation of the physical ionization process are 
excited state proton transfer in the solid phase before desorption and gas-phase proton transfer in the gas plume after photoionization of the matrix molecules. ${ }^{7}$ The basic MALDI principle is visualized in Figure 1.1.

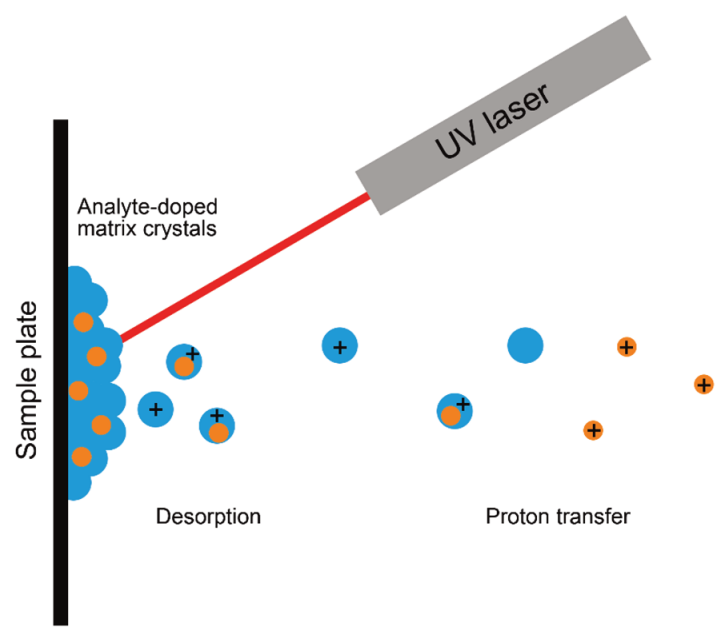

Figure 1.1 Matrix-assisted laser desorption ionization mechanism (adapted from de Hoffmann et al. ${ }^{4}$ )

The MALDI matrix minimizes damage from the laser by absorbing the energy and makes the energy transfer from laser to analyte more efficient. This leads to higher sensitivity compared to other laser ionization techniques. Because the MALDI process is driven by the energy absorption of the matrix, it can ionize molecules with very large molecular weights. MALDI produces predominantly singly charged ions in a pulsed way. Therefore, a MALDI source is often combined with a time-of-flight (TOF) mass analyzer due to its high mass range and its ability to handle bundles of ions. In addition, MALDI matrix can be applied directly on tissue and is a successful technique to study a molecule's distribution within this tissue. MALDI imaging will be discussed in paragraph 4 of this chapter.

\section{Electrospray ionization}

Shortly after the development of MALDI in 1988, Fenn et al. revolutionized the mass spectrometry field in 1989 by the development of electrospray ionization (ESI) ${ }^{8}$. Multiply charged ions were obtained from proteins and peptides, which allows the detection of proteins and peptides with MS instruments that have a limited mass range. Other advantages are the very efficient ionization of molecules, its production at atmospheric conditions, and the easy incorporation with liquid-based separation techniques, such as liquid chromatography and capillary electrophoresis. 
ESI produces ions by the application of a strong electric field to a liquid passing through a capillary tube at a potential difference of 1-5 $\mathrm{kV}$ between the capillary and the inlet cone. At a low flow rate, gas flows coaxially to prevent dispersion of the spray, which then passes through a curtain of heated inert gas (often nitrogen) to help remove the last solvent in the droplets. The electric field causes a charge accumulation at the liquid surface at the end of the capillary leading to highly charged droplets. When the surface tension is broken, the highly charged droplets change into a 'Taylor cone' and ESI is initiated. Depending on the surface tension of the chosen solvent, the optimal spray potential varies. After the formation of the 'Taylor cone', the droplet elongates under the accumulative force of charge. The solvent in the droplets evaporates and Coulombic explosion results in smaller charged droplets. Solvent evaporation will continue until the charge on the droplet surface is large enough. In the final ionization step, two major mechanisms exist: the ion evaporation model (IEM) and the charge residue model (CRM). CRM, where solvent evaporation occurs until one multiply charged ion is formed, is the dominant model for the formation of large multiply charged ions. In IEM, the dominant model for small molecules, ions are desorbed from the small droplet due to the strong electric field in the ion source ${ }^{4,8,9}$. The ESI mechanism is schematically depicted in Figure 1.2.

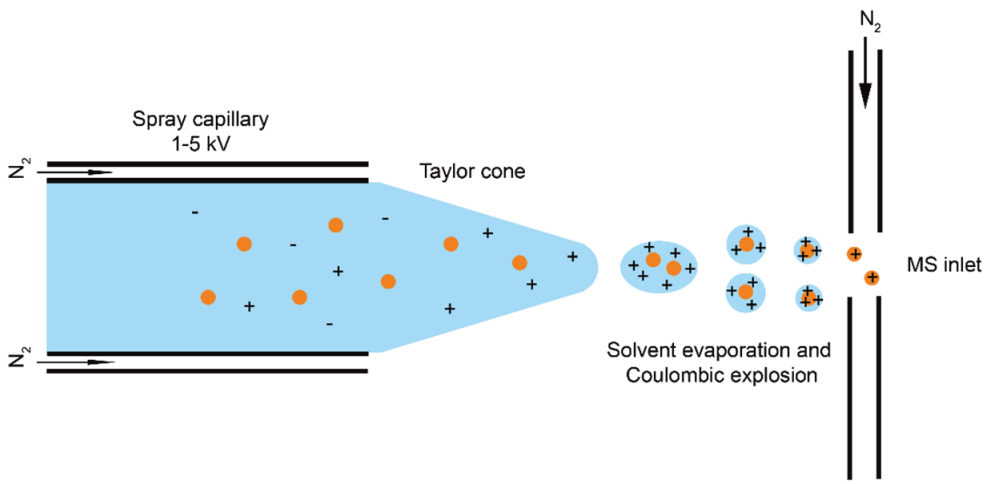

Figure 1.2 Electrospray ionization mechanism (adapted from de Hoffmann et al. ${ }^{4}$ )

Because desorption of ions takes place at the surface of the droplet, higher sensitivity is achieved for more lipophilic molecules. Molecules that are present in the center of the droplet can sometimes be masked by those lipophilic molecules at the surface. Larger molecules, such as proteins, will not desorb at the surface but are freed after evaporation of the solvent. As mentioned earlier, ESI produces multiply charged ions from molecules if they contain multiple ionizable sites in there molecular structure. 
These multiply charged species are recognized in the mass spectrum because their isotopic peaks differ <1 Da.

\subsubsection{Mass analyzers}

The performance of a mass analyzer is often expressed in terms of speed, sensitivity, mass accuracy, mass range, dynamic range, mass resolution, and mass resolving power. The speed of a mass analyzer is the rate at which it measures over a particular mass range. Sensitivity expresses the efficiency of ion transmission and detection. Mass accuracy refers to the error between the theoretical $\mathrm{m} / \mathrm{z}$ value and the measured $\mathrm{m} / \mathrm{z}$ of an ion. Mass range is the $\mathrm{m} / \mathrm{z}$ range limit over which the analyzer can measure ions. Dynamic range expresses the ratio between the largest and smallest detectable signal. Mass resolution is the ability to separate two ions with a small $m / z$ difference. This $m / z$ difference is determined between two ions of the same height, which is rarely the case in a recorded mass spectrum. Therefore, mass resolving power is the preferred term to use with regards to its separation power. Mass resolving power is expressed by the observed $\mathrm{m} / \mathrm{z}$ value divided by the peak width at half height. The greater the mass resolving power, the improved capacity to separate ions with a smaller $\mathrm{m} / \mathrm{z}$ difference. The terms mass resolution and mass resolving power are confusingly used in literature, however, in this thesis, we prefer to use mass resolving power to express the mass analyzer's separation power.

All mass analyzers separate ions under high vacuum conditions to prevent ions to collide and possibly lose their charge. The majority of MS instruments contain one or more of the following mass analyzers: Quadrupole ${ }^{10}$, Linear ion trap ${ }^{11}$, Time-of-flight ${ }^{12}$, Orbitrap $^{13}$, and Fourier Transform Ion Cyclotron Resonance (FT-ICR) ${ }^{14}$. In this introduction, we will focus on the mass analyzers used in this thesis.

\section{Quadrupole mass analyzer}

A quadrupole mass analyzer consists of four parallel circular or hyperbolic rods. Opposite rods have either a positive or a negative direct current (DC) potential applied at which an alternating current $(A C)$ is superimposed. This $A C$ is in the radio frequency (RF) region. In simple words, this means that if a positive ion enters the quadrupole along the $z$ axis, it is drawn towards a negative rod. Collision of the ion with the rod will neutralize the ion and terminates its detection. When the potential changes before the ion hits the rod, the ion will change its trajectory. A quadrupole mass analyzer is depicted in Figure 1.3. 


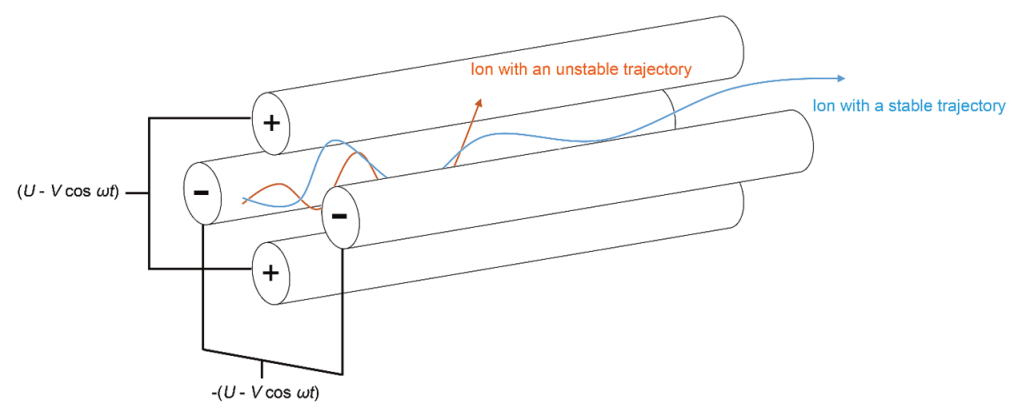

Figure 1.3 Quadrupole mass analyzer (adapted from Niessen et al. ${ }^{3}$ )

The RF/DC ratio determines which ions have a stable trajectory through the quadrupole. The ion trajectory is described by the Mathieu equation (see Equation 1) ${ }^{10}$ :

$$
\frac{\mathrm{d}^{2} u}{\mathrm{~d} \xi^{2}}+\left(a_{u}-2 q_{u} \cos 2 \xi\right) u=0 \quad a_{u}=\frac{8 e U}{m r_{0}{ }^{2} \omega^{2}} \quad q_{u}=\frac{4 e V}{m r_{0}{ }^{2} \omega^{2}}
$$

$u$ stands for the $x$ and $y$ axis coordinates, $\xi$ is $\omega t / 2, t$ is time, $e$ is the electron charge, $U$ is the DC voltage, $V$ is the RF voltage, $m$ is the mass of the ion, $r_{0}$ is the radius between the rods, and $\omega$ is the RF frequency. The parameters $a_{u}$ and $q_{u}$ are proportional to $U$ and $V$, respectively. To simplify this equation, it is important to recognize the relationship between the coordinates of the ion and time. As long as the coordinates of the ion are less than $r_{0}$, the ion will not hit the rod and be able to pass through the quadrupole. Each quadrupole instrument has a constant $r_{0}$ and $\omega$ which means that for an ion with mass $m, x$ and y can be determined as a function of $U$ and $V$ over a certain period of time. This is presented in a Mathieu stability diagram as shown in Figure 1.4. 


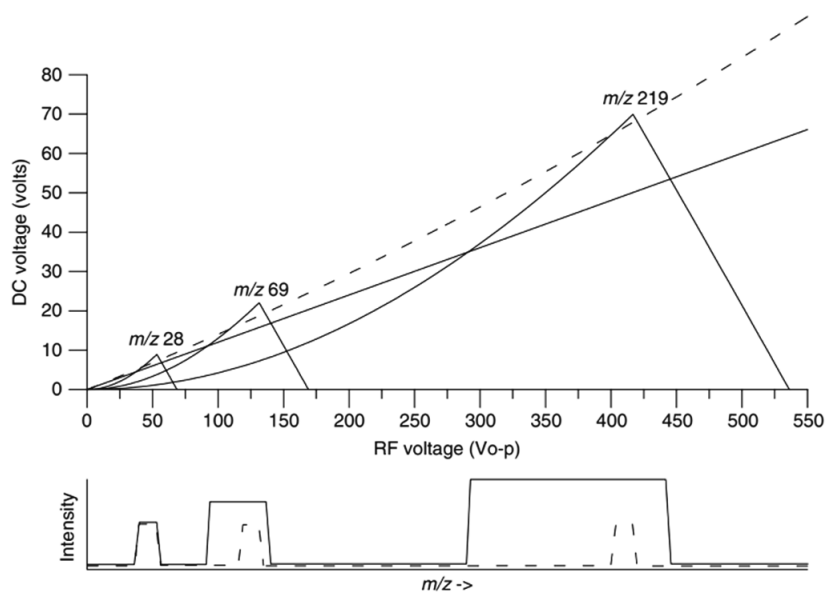

Figure 1.4 Mathieu stability diagram (Figure taken from Kero et al. ${ }^{10}$ )

A quadrupole is often used in three different modes. The first mode is the full scan mode in which the RF/DC ratio is fixed while it scans over a certain mass range. This mode provides a mass spectrum that contains nominal $\mathrm{m} / \mathrm{z}$ values. In the second 'RF only' mode, $U=0$ which allows the transmission of all ions through. This way the quadrupole is used to focus the ion bundle. In the last mode, which is called selected ion monitoring (SIM), only one ion is selected for transmission to the detector by keeping the DC/RF values at a fixed value. Sequential selection of multiple ions is possible with the switching time is often $\sim 5 \mathrm{~ms}$.

\section{Time-of-flight mass analyzer}

In a linear time-of-flight (TOF) mass analyzer, ions are initially accelerated by an electric field in a pulsed manner followed by a separation based on individual velocities as they drift through the field-free region ${ }^{4,15}$. When an ion with mass $m$ and charge $z$ is accelerated by a potential $V_{s}$, this energy is converted into the kinetic energy of the ion. The velocity $v$ is determined by the following equations (Equation 2 and 3):

$$
E_{k}=\frac{m v^{2}}{2}=z V_{s} \quad v=\sqrt{\frac{2 z V_{s}}{m}}
$$

The flight time $t$ and $m / z$ value are then calculated by considering the velocity of the ion and the flight tube distance $L$ :

$$
t^{2}=\frac{m}{z}\left(\frac{L^{2}}{2 V_{s}}\right)
$$


Most TOF instruments contain a reflectron to improve its mass resolving power. The reflectron, which consists of a set of serial grids situated behind the field-free region, acts as an ion mirror by sending the ions back through the flight tube. Faster ions, with more kinetic energy and more velocity, will penetrate the reflectron deeper than slower ions of the same $\mathrm{m} / \mathrm{z}$. Due to the reflectron, the total flight distance is prolonged and, additionally, the kinetic energy dispersion of ions with the same $\mathrm{m} / \mathrm{z}$ is corrected for (in other words 'time-focused'). Both of these features are leading to higher mass resolving power and better mass accuracy of the TOF analyzer. Figure 1.5 shows a reflectron TOF mass analyzer ${ }^{12}$.

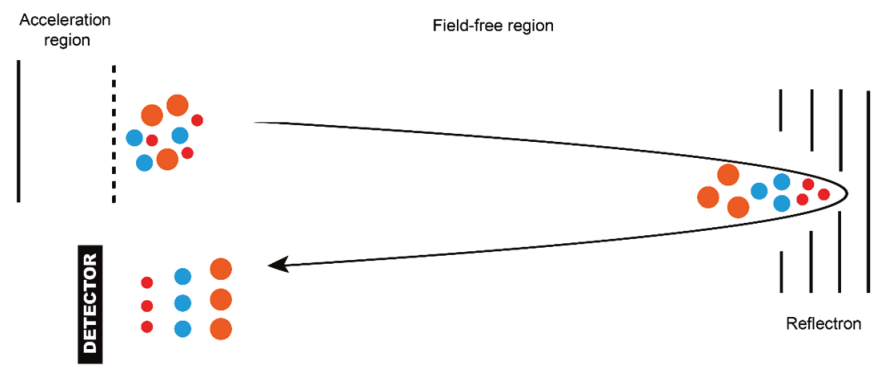

Figure 1.5 Time of flight mass analyzer (adapted from Mamyrin et al. ${ }^{12}$ )

\section{Trapped ion mass analyzers}

This section briefly explains two trapped ion mass analyzers: a linear ion trap (LIT) and an orbitrap mass analyzer. The mechanism of a LIT is similar to that of the quadrupole mass analyzer ${ }^{11}$. In a LIT, the four parallel rods have lenses on both ends to repel ions inside the rods, either at positive (for positive ions) or negative (for negative ions) potential. Ions are cooled by collisions with an inert gas $\left(\mathrm{N}_{2}\right)$ moving along the z-axis along the rods while oscillating in the xy-plane. In addition to the end lenses, the four rods are divided into three segments: two segments at the end have a DC voltage applied to repel ions while the middle segment is kept at RF-only potential. Ions are ejected from the LIT by applying additional AC voltages between the rods and the end electrode (the exit lens).

The orbitrap mass analyzer was introduced by Makarov et al. in 2000 and is an electrostatic ion trap that uses Fourier transform to generate mass spectra ${ }^{13}$. It consists of an internal (spindle-shaped) and external (barrel-shaped that is cut into two equal parts) electrodes. Ions are injected into the trap via this opening in the external 
electrode. The internal electrode has an electrostatic DC voltage of several kilovolts applied to (negative for positive ions and vice versa) and the external electrode is at ground potential. Ions with several kiloelectronvolts kinetic energy are injected and start to rotate around the internal electrode while moving along the z-axis. The frequency of oscillations is directly linked to the $\mathrm{m} / \mathrm{z}$ ratio and is independent of the kinetic energy of the ions. The broadband current induced by the oscillating ions is converted by a Fourier transform to individual frequencies and intensities in the mass spectrum. An extensive explanation of the orbitrap mass analyzer can be found in this reference ${ }^{16}$.

\subsubsection{Ion detection}

After separation of the ions based on their $\mathrm{m} / \mathrm{z}$ value in the mass analyzer the individual ions need to be detected. The travelling ions need to be converted into an electric current which is, ideally, proportional to their abundance. Because the number of ions that leave the mass analyzer is very low, they need to be amplified to obtain a sufficient signal. This is often done by an electron multiplier, which consists of multiple conversion dynodes (electrodes) at a high potential. The first dynode is of opposite potential than the ions of interest. When e.g. positive ions hit the first conversion dynode, secondary particles are generated such as negative ions and electrons. Negative ions result in positive ions after hitting the first conversion dynode, which are then converted into negative ions and electrons. This electron amplification continues until a significant number of electrons are generated. These secondary electrons are collected by an anode at the end of the detector and the current is measured. In some instruments a photomultiplier is used for ion detection, which transfers the secondary electrons into photons by accelerating them towards phosphorescent screen. A photomultiplier detects these photons and converts them into an electric current. The main advantage of a photomultiplier over an electron multiplier is its lifetime and fast response time. In addition, for fast data acquisition in MS, fast electronics such as analog-to-digital converters (ADCs) are necessary. An ADC receives ion arrival times and number of ions (ion intensity) and converts this information into a digital output to generate a mass spectrum. Detection in an FT-ICR or orbitrap is more complicated and will not be discussed in this introduction. More information can be found in the following references ${ }^{13,14,17,18}$.

\subsubsection{Tandem mass spectrometry}

MS not only allows the detection of molecules in a complex mixture but can also be used to identify them. Structural identification of molecules requires fragmentation of their ions which can be done by tandem mass spectrometry (MS/MS $)^{3,4,19}$. In a tandem 
mass spectrometer, two or more mass analyzers are present with a fragmentation cell in between. MS/MS can be distinguished into two types: in space and in time ${ }^{20}$. MS/MS in space occurs in an instrument that contains two mass analyzers. Identification is performed by using the first mass analyzer to select the ion of interest (e.g. precursor ion), fragment this ion into structurally identifiable fragment ions (e.g. product ions), measure the $\mathrm{m} / \mathrm{z}$ values of these fragment ions in the second mass analyzer. MS/MS in time is performed in an instrument that contains one mass analyzer, often in trappedion mass spectrometers. The precursor ions are selected in the mass analyzer (all other ions are eliminated from the trap), collision gas is introduced to generate fragment ions, and afterwards fragment ions are measured in the same mass analyzer. This successive way of analyzing can be repeated for several times to obtain fragments of fragment ions (e.g. MS $)$.

Although the general principle is the same, different fragmentation methods are available which leads to different fragment ions. The fragmentation method used in this thesis is called collision-induced dissociation (CID $)^{21,22}$. In the fragmentation cell, collisions between the precursor ions and an inert collision gas (often $\mathrm{N}_{2}$ ) cause the kinetic energy to be converted into internal energy which brings the ion into an excited state leading to decomposition/fragmentation.

MS/MS in space is often executed on two MS instruments: a triple quadrupole mass spectrometer (QqQ) and a quadrupole time-of-flight mass spectrometer (Q-TOF). The MS/MS capabilities of both instruments are described in more detail below.

\section{Triple quadruple mass spectrometer}

A triple quadrupole $(\mathrm{QqQ})$ instrument consist of two quadrupole mass analyzers (Q1 and Q3) with a fragmentation cell (q) in between. Historically, the instrument was built with three quadrupoles the second of which was used as q. In modern instruments, $q$ is replaced by a higher-order multipole, which makes the term triple quadrupole not accurate. This instrument is, therefore, often called a tandem quadrupole mass spectrometer. However, in this thesis, we refer to this instrument as a triple quadrupole in consistency with common practice within the MS community ${ }^{3}$.

As described previously in this introduction, a quadrupole mass analyzer can be used in three operational modes. When two mass analyzers are placed in one MS instrument, this allows for four different QqQ MS/MS modes presented in figure 1.6: i) product ion scan (PROD), ii) precursor ion scan (PREC), iii) neutral loss scan (NL), and iv) selected reaction monitoring $(S R M)^{23}, 24$. In PROD, a precursor ion is selected in Q1, fragmented in $\mathrm{q}$, and fragment ions are scanned in Q3. In PREC, the precursor ions that contain one 
specific fragment ion (selected in Q3) are scanned in Q1. In NL, precursor ions in Q1 and fragment ions in Q3 are scanned with an offset corresponding to a neutral fragment loss from the precursor ions. In SRM, Q1 and Q3 are both used in SIM mode, which results in a specific precursor ion-fragment ion transition. This way all other ions are removed and focus on this transition can be held for a longer period of time which leads to a significant boost in sensitivity. SRM is often applied on multiple transitions and is, therefore, referred to as multiple reaction monitoring (MRM). In MRM, one should be aware of the cross-talk between multiple MRM transitions with the same fragment ions. Multiple transitions from the same precursor ion can confirm its identification. Fast switching times between MRM transitions allows for sensitive high throughput screening of large amounts of molecules. In addition, a QqQ instrument offers a high dynamic range which makes it very suitable for molecular quantification.

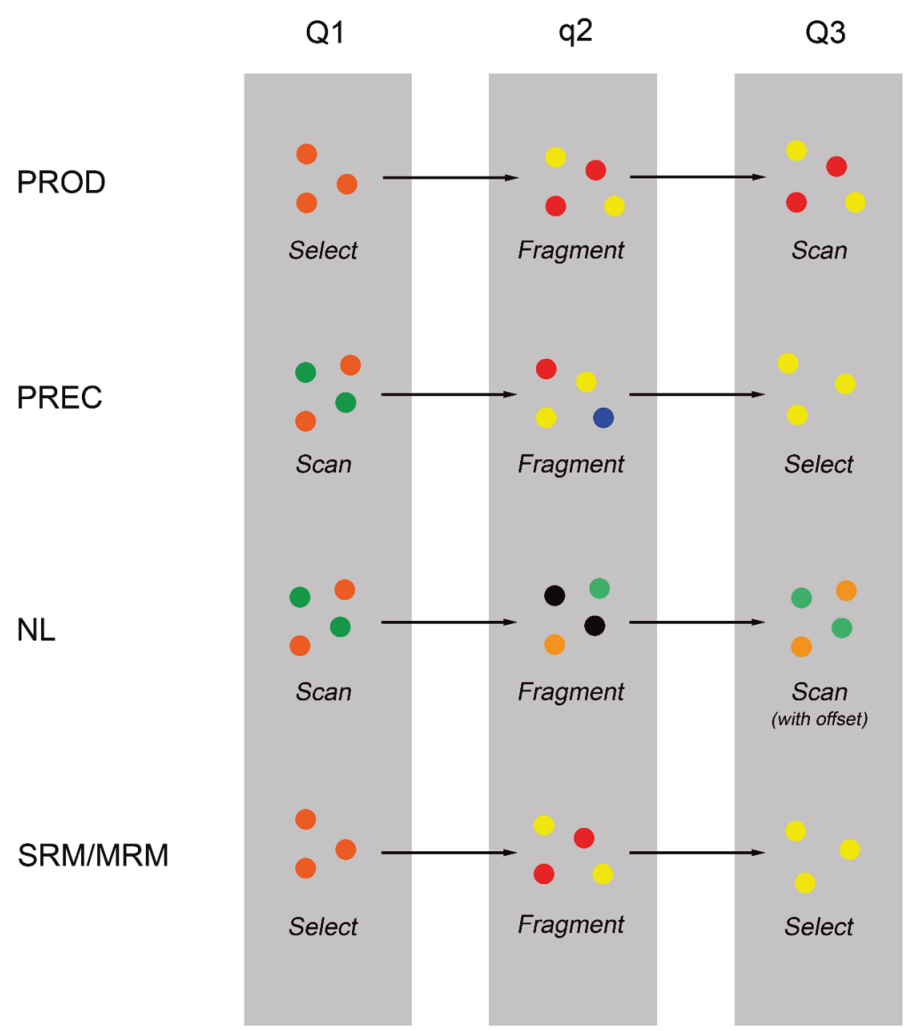

Figure 1.6 QqQ tandem mass spectrometry modes: PROD, PREC, NL and SRM/MRM (adapted from Brügger ${ }^{25}$ ) 


\section{Quadrupole time-of-flight mass spectrometer}

A quadrupole is combined with a reflectron TOF analyzer in a Q-TOF instrument separated by a fragmentation cell to perform MS/MS with high mass resolving power ${ }^{26}$. In full scan mode, both the quadrupole and fragmentation cell are used in RF only mode. In MS/MS mode, the quadrupole is used for precursor ion selection and the TOF is, after fragmentation, used for high mass resolving power scanning of fragment ions. Because a TOF analyzer is a pulsed system that requires ions to be introduced in bundles, the combination with a continuous ion beam from ESI requires orthogonal acceleration in the instrumental setup ${ }^{27}$. Orthogonal acceleration transforms a continuous ion beam into a pulsed process by applying an electric field in the orthogonal direction of the ion beam and accelerates the ions into the TOF analyzer. Because of this high mass revolving power scanning, a Q-TOF instrument is a very suitable instrument for structural identification of unknown molecules.

\subsection{Structural analysis}

MS/MS is necessary to determine the molecular structure of an unknown compound ${ }^{28-}$ 30. Hsu and Turk have shown structural characterization for multiple lipid classes by direct infusion combined with MS/MS ${ }^{31-39}$. Even though the information provided by MS/MS is essential, some lipid isomers are still challenging to identify. In general, isomers are different molecules with the same chemical formula and, therefore, the same exact mass ${ }^{40}$. In some unknown cases, even fragments from isomers are identical and could not be separated by MS. The term isomers should not be confused with isobars, which refers to two molecules with the same nominal mass but not the same molecular formula, thus different exact mass. Isobars could be isolated using MS with high enough mass resolving power while MS alone cannot isolate isomers. In that perspective, isomers can be divided into two groups: structural isomers (same molecular formula, different atom sequence) and stereoisomers (same molecular formula and atom sequence, different 3D orientation of atoms) ${ }^{40}$. Structural isomers can often be separated by MS/MS but stereoisomers are not. These molecules require complementary separation to MS/MS to perform structural analysis, such as nuclear magnetic resonance (NMR) spectroscopy ${ }^{41}$. This is quite challenging because NMR requires a much more concentrated/pure sample and is very time-consuming. In addition to resolving stereoisomers by NMR, biological samples are very complex and their mass spectra show isobaric interference due to this complexity. Therefore, they require cleanup/concentration before NMR/MS identification, which can be achieved by complementary separation prior to NMR/MS analysis. The complementary separation tools that frequently used are gas chromatography $(\mathrm{GC})^{42,43}$. liquid chromatography $(\mathrm{LC})^{44-46}$, capillary zone electrophoresis (CZE $)^{47-49}$, and ion mobility spectrometry $(\mathrm{IMS})^{50-}$ 
54. These techniques simplify a mass spectrum and, as a result, may lead to structural identification. In this thesis, two separation techniques are further explained: IMS and LC.

\subsubsection{Ion mobility spectrometry}

Ion mobility spectrometry (IMS) separates gas-phase ions based on their size-to-charge ratio as they are moving through a buffer gas by an applied electric field ${ }^{55-57}$. The ion's velocity $v$ is expressed by the multiplication of its mobility $K$ and the electric field $E$ (Equation 4):

$$
v=K E
$$

Under low field conditions, the ion mobility constant $K$ is related to the collision crosssection $\Omega$ of the ion by the Mason-Schamp equation (Equation 5$)^{58,59}$.

$$
K=\left(\frac{3 q}{16 N}\right)\left(\frac{2 \pi}{k T}\right)^{1 / 2}\left(\frac{m+M}{m M}\right)^{1 / 2}\left(\frac{1}{\Omega}\right)
$$

in which $q$ is the ion's charge, $N$ is the number density of the buffer gas, $k$ is the Boltzmann's constant, $T$ the absolute temperature, $m$ is the mass of the buffer gas and $M$ is the mass of the ion.

IMS is combined with MS since the 1960s. Four different IMS techniques are distinguished: i) drift tube ion mobility spectrometry (DTIMS ${ }^{60}$, ii) travelling wave ion mobility spectrometry (TWIMS) ${ }^{58,61,62}$, iii) trapped ion mobility spectrometry (TIMS) ${ }^{63,64}$, and iv) field asymmetric ion mobility spectrometry (FAIMS) ${ }^{65-68}$. The basic principles of all four IMS techniques are visualized in Figure 1.7. 
DTIMS
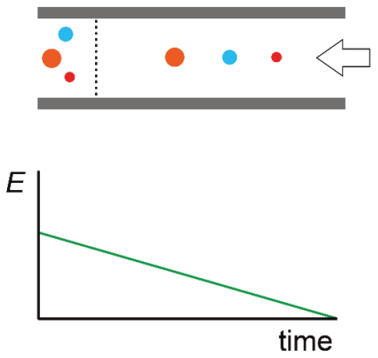

TIMS
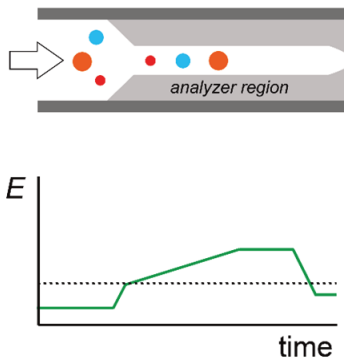

TWIMS
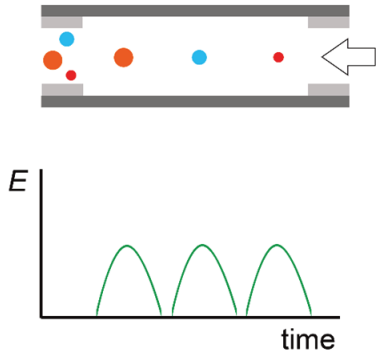

FAIMS
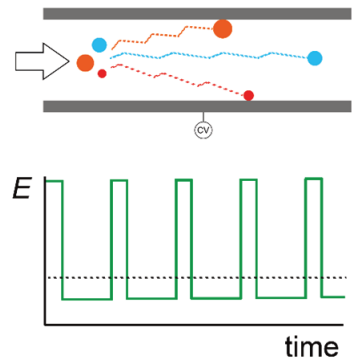

Figure 1.7 Four main types of ion mobility spectrometry: drift tube ion mobility spectrometry (DTIMS), travelling wave ion mobility spectrometry (TWIMS), trapped ion mobility spectrometry (TIMS), and field asymmetric ion mobility spectrometry (FAIMS).

DTIMS measures the time an ion moves through a buffer gas while a low electric field is applied ${ }^{69}$. DTIMS can be used under two conditions: atmospheric pressure and reduced pressure. Reduced pressure DTIMS has a better ion transmission to the mass spectrometer than DTIMS at ambient conditions but loses resolving power versus atmospheric pressure DTIMS ${ }^{55}$. TWIMS is the second IMS type in which ions pass through the buffer gas accelerated by symmetric potential waves. Overall, TWIMS offers better ion transmission efficiency due to the higher vacuum conditions ${ }^{58}$. TIMS separates ions based on a reversed principle: ions are carried into the IMS cell by a carrier gas and trapped by the electric field ${ }^{63,70}$. All three described IMS techniques operate at low-tomoderate electric field conditions, which makes the Mason-Schamp equation applicable and the linear $v$ to $E$ relationship classifies them as linear IMS. FAIMS is considered nonlinear IMS because separation takes place when applying an asymmetric wave field that rapidly applies high and low electric fields. This rapid field switching separates ions 
while transported between two planar electrodes by a carrier gas ${ }^{65,71}$. FAIMS has proven to be far more orthogonal to MS than linear IMS techniques ${ }^{72}$.

\subsubsection{Liquid chromatography}

Chromatography covers a group of analytical methods that allow the separation of isomeric compounds in complex biological mixtures. The technique is established in analytical chemistry as in some cases chromatography is the only applicable tool with sufficient separation power. The basic principle is the same for all chromatographic separations. A sample is dissolved in a mobile phase, either a gas (GC) or a liquid (LC), and passed through a stationary phase ${ }^{73}$. In LC, the stationary phase is a packed column with particles. Because analytes in the sample can only move when they are in the mobile phase; they are more or less retained dependent on the time they spend in the stationary phase. For sufficient separation power, two analytes should be retained for a certain amount of time. However, the more time an analyte spends on a column, the broader its peak will be. Chromatographic separation of similar molecules is affected by chemical and physical parameters (of the column) that improve peak separation while limiting the amount of peak broadening. The performance of a chromatographic column, so-called column efficiency, is expressed by two terms: plate height $H$ and number of theoretical plates $N$. A theoretical plate is a hypothetical stage at which both phases are at equilibrium with each other. The more theoretical plates, the more efficient is the column. The two terms are related to each other by the Equation $6{ }^{73}$ :

$$
N=\frac{L}{H}
$$

in which $L$ is the column length. A low plate height is desirable and should be considered during column tests. The Van Deemter plot and equation (Equation 7) express the plate height by three terms that represent column processes during the chromatographic interaction: eddy diffusion term $A$, longitudinal diffusion term $B / u$ and mass transfer term $C \cdot u^{74-76}$

$$
H=A+\frac{B}{u}+\left(C_{M}+C_{S}\right) \cdot u
$$

$A$ is the eddy diffusion coefficient, $B$ is the longitudinal diffusion coefficient, $C$ the sum of the mass transfer coefficients of both phases and $u$ the linear velocity of the mobile phase $(\mathrm{cm} / \mathrm{s})$. Eddy diffusion reflects the possibility of a molecule to diffuse in the column packing if the mobile phase flow rate is not optimal which leads to peak broadening. Longitudinal diffusion is the migration of the sample to more dilute regions of the 'sample zone' on both sides. At higher flow rates, this term has minimal effect 
on the plate height. The mass transfer term is defined by two mass transfer coefficients for both the mobile $\left(C_{M}\right)$ and stationary phase $\left(C_{S}\right)$, which reflect both the travelling time through the mobile phase and the time required to be adsorbed to/desorbed from the stationary phase. The higher the flow rate, the less migration time for the analyte to interact with the stationary phase. $C_{M}$ is proportional to the square of the particle diameter and column diameter. A narrower column with smaller particles allows for more interaction (and therefore retention) with the stationary phase and can facilitate higher flow rates. Since the late 1960s, LC systems improved so they could perform at higher pressures (from 250 bar up to 1000 bar), referred to as high-performance liquid chromatography (HPLC) systems, which were further upgraded to ultra highperformance liquid chromatography (UHPLC or UPLC) systems in $2004^{77}$. As UHPLC systems became the standard for routine chromatographic analysis, these systems are abbreviated as LC in literature.

\subsection{Mass spectrometry imaging}

MALDI based Mass spectrometry imaging (MSI) was first reported by Caprioli et al. in 1997 in which proteins and peptides were imaged in biological tissue by MALDI-TOF ${ }^{78}$. Since its introduction, MSI has grown into an indispensable field for label-free spatial MS profiling directly from a surface and is recognized as a valuable tool in pharmaceutical ${ }^{79-83}$, biomedica| ${ }^{84-87}$, and clinical research ${ }^{88-90}$. MSI is used to study the distribution of not only endogenous molecules like proteins and lipids but also exogenous compounds such as pharmaceuticals, all on very complex surfaces ${ }^{91-94}$. These molecules can be mapped at the sub-cellular level using an ionization tool that can provide high spatial resolution and even larger surfaces like complete organs and whole biological systems can be imaged ${ }^{95}$. In addition to the MS performance characteristics discussed previously, important features of an MSI technique are its spatial resolution (often expressed in pixel size) and its speed (often expressed in pixels per second). The higher spatial resolution is used, the longer analysis time is required to maintain the same ion detection level. This is a constant consideration for the application in clinical and pharmaceutical research in which fast and high throughput analysis is required. The basic MSI workflow (Figure 1.8) consists of four building blocks: spatial ionization, MS analysis, single pixel mass analysis, and the construction of $\mathrm{m} / \mathrm{z}$ specific intensity heat maps (MS images). 


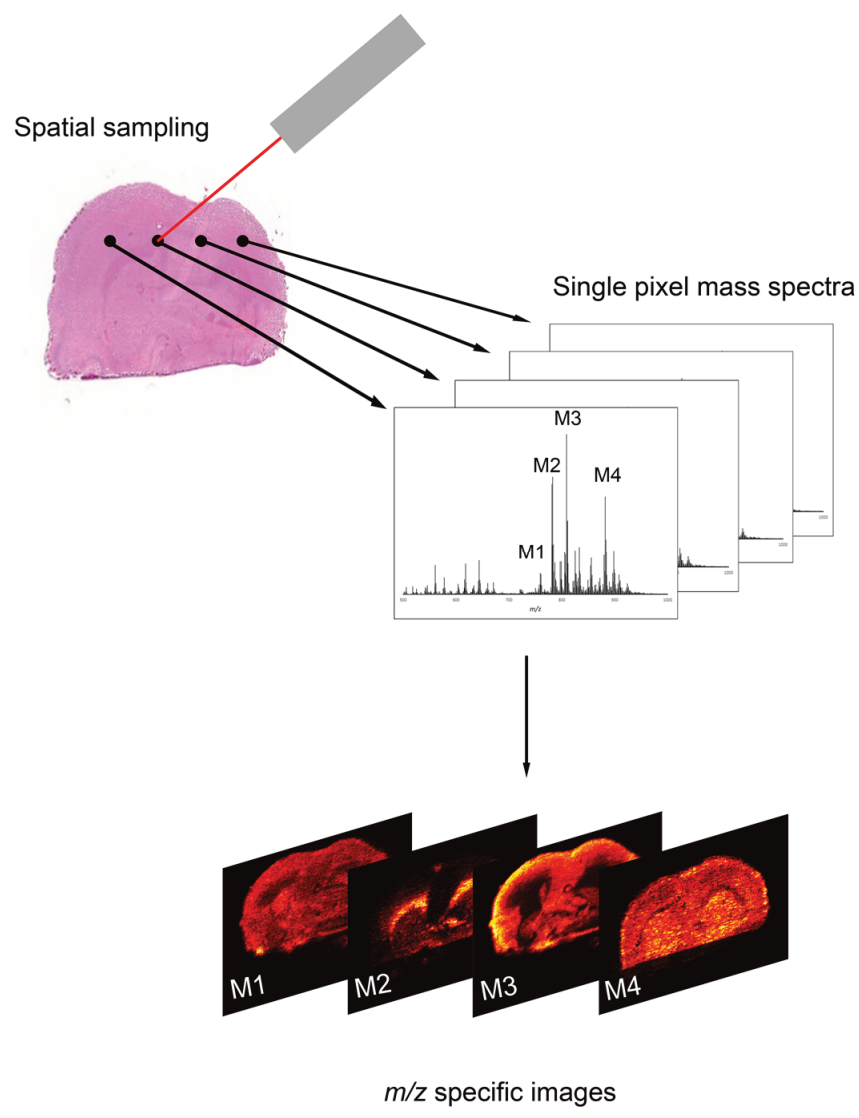

Figure 1.8 Mass spectrometry imaging workflow.

A variety of MSI ionization techniques is available, such as MALDI ${ }^{96}$, secondary ion MS $(\mathrm{SIMS})^{97,98}$, and desorption electrospray ionization $(\mathrm{DESI})^{99}{ }^{100}$. These three MSI ionization technique have been groundbreaking for the MSI field and deliver complementary characteristics in terms of target analytes, spatial resolution and sample preparation. MALDI-MSI is by far the most applied MSI technique since it is applicable to many different compound classes. After the matrix application, which is sprayed or sublimed over the sample surface, a MALDI laser is used to spatially ionize molecules present in the sample. The spectra in the obtained MS images correlate with the X-and $Y$-coordinates of the laser shots ${ }^{101}$. Spatial resolution in MALDI-MSI is typically around 20-30 $\mu \mathrm{m}$, however, a minimal pixel size of $>5 \mu \mathrm{m}$ has been reported ${ }^{102-104}$. MALDI-MSI achieves high sensitivity for a wide range of molecules but its main limitation is the timeconsuming matrix application. The matrix application step can lead to analyte 
delocalization and suppression of ions due to the matrix crystals ${ }^{105}$. Research has extensively focused on the optimization of sample preparation protocols that involve tissue washing ${ }^{106}$, on-tissue digestion ${ }^{107,}{ }^{108}$, on-tissue derivatization ${ }^{109-111}$, and sublimed matrix application in combination with recrystallization ${ }^{112}$. All studies targeted the improvement of molecular discovery or spatial resolution through the improvement of sample preparation methodology.

SIMS uses a primary ion beam to generate secondary ions from a surface ${ }^{113}$. The primary ion beam can be focused to $a<100 \mathrm{~nm}$ spot size and is ideal for sub-cellular imaging. SIMS often results in fragmentation of molecules due to the high amount of energy from the primary ion beam on a very small surface, which makes detection large biomolecules difficult. As a result, SIMS is the preferred MSI tool for imaging small molecules and elements when high spatial resolution is required ${ }^{95}$. Ambient MSI techniques, including DESI, are used in the research presented in this thesis and are further discussed in more detail.

\subsubsection{Ambient mass spectrometry imaging}

Several MSI ionization techniques perform at atmospheric pressure. These ambient ionization techniques do not require matrix application and are compatible with samples that cannot be placed under vacuum conditions ${ }^{114-116}$. Ambient MSI ionization often uses a solvent to extract molecules of interest from the sample prior to ionization or desorb and ionize molecules directly from a substrate. Although lower sensitivity is observed for larger biomolecules, the ability to adapt the solvent composition to the targeted compound (classes) is a major advantage of ambient MSI ${ }^{100}$. Common ambient MSI ionization techniques are: desorption electrospray ionization (DESI) ${ }^{99}$, 117, 118 , laser ablation electrospray ionization (LAESI) ${ }^{119}$, 120, liquid extraction surface analysis (LESA) ${ }^{121}$, 122, which is often referred to as liquid microjunction surface sampling probe (LMJ/SSP; flow probe) 123-125, nanospray desorption electrospray ionization (nano-DESI) ${ }^{126,127}$, and laser ablation liquid capture surface analysis (LA/LCSA) ${ }^{128,}{ }^{129}$. Laskin et al. provided an informative review, which explains multiple ambient MSI techniques ${ }^{130}$.

\section{Desorption electrospray ionization}

DESI is the most widely applied ambient MSI technique and was first reported in $2004^{117}$, 118. Its mechanism combines ESI and desorption ionization. DESI uses a supersonic electrospray jet which is directed to a sample surface. A potential of 1-5 kV is applied to generate primary charged solvent droplets that are focused towards the sample by a high speed nebulizing gas (e.g. $\mathrm{N}_{2}$ ). These primary charged droplets form a small thin liquid layer on the sample in which extracted molecules are desorbed. Secondary 
charged droplets are obtained due to the continuous bombardment of the electrospray into the thin liquid layer. The solvent in these secondary charged droplets evaporates and molecules are ionized. This mechanism is illustrated in Figure 1.9.

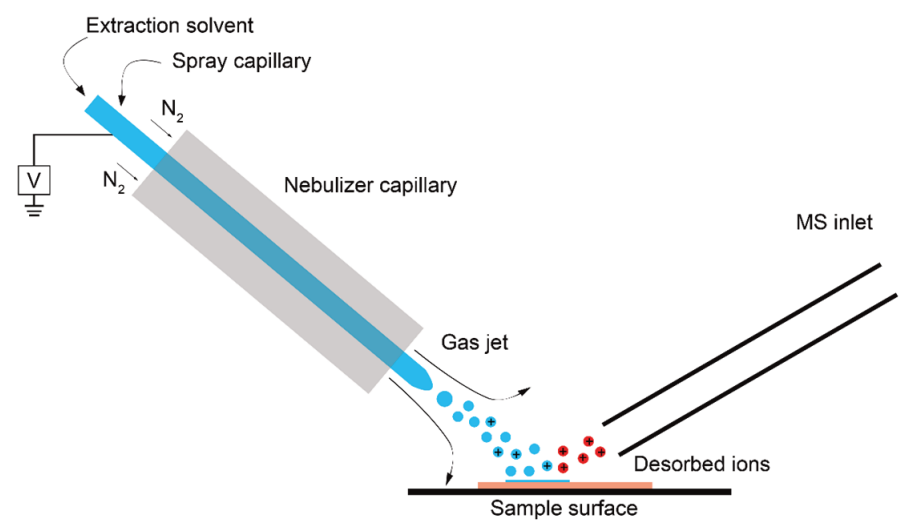

Figure 1.9 A schematic overview of desorption electrospray ionization.

DESI has several advantages compared to MALDI. First, no matrix application is needed which minimizes the sample preparation time. Second, multiply charged ions are generated which extends the mass range (similar to ESI). In addition, the DESI solvent can be targeted to the analytes of interest. DESI is nondestructive to the tissue sample which allows complementary MALDI-MSI analysis afterwards ${ }^{131}$. As with all ambient ionization techniques, DESI does not require vacuum condition. Pixel sizes in DESI are often around 50 um with a minimal $>20$ um reported ${ }^{132}$. The DESI source can move fast and therefore can scan samples with high throughput $(<5 \mathrm{~min} /$ analysis) in a clinical assessment ${ }^{133,134 .}$

\section{Liquid extraction surface analysis}

LESA was first described by Kertesz and coworkers in 2010 and uses a liquid droplet to extract molecules from a surface prior to direct MS analysis via nanoelectrospray ionization ${ }^{135}$. LESA has similar advantages as other ambient MSI techniques as it requires minimal sample preparation and extraction solvent can be modified to the target analytes ${ }^{136}$. However, spatial resolution of LESA is around 1-1.5 mm in which several histological features are lost compared to other MSI techniques. For this reason, LESA is often applied as a spatial profiling tool. The major advantage of LESA is that this spatial extraction unit can be combined with chromatographic separation, such as thin 
layer chromatography or even column based LC, to provide an additional dimension complementary to $\mathrm{MS}^{123,137 .}$

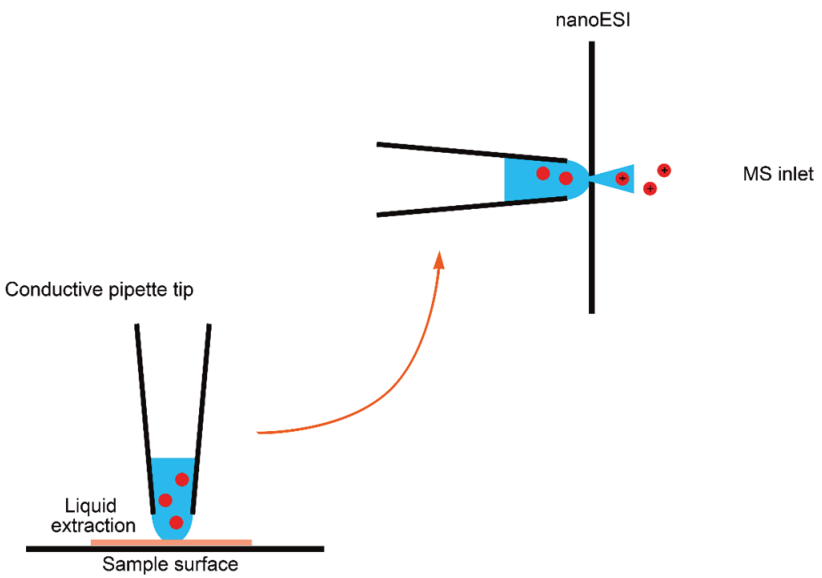

Figure 1.10 The principle of liquid extraction surface analysis.

\subsection{Pharmaceutical development}

In drug discovery and drug development, the main goal is finding suitable drug candidates that can inhibit or activate a pathway or protein to have a positive effect in a disease state ${ }^{82}$. Pharmacology studies various interactions of a drug candidate with a biological system by pharmacokinetics (what the system does to the drug; PK) and pharmacodynamics (what the drug does to the system; PD) ${ }^{138}$. In general, the desired effect is that the potential drug is distributed, and if required metabolized, to the target site and bound to the biological receptors present for a certain amount of time at a high enough concentration ${ }^{139}$, then released and excreted as soon as possible, all without initiating a toxic effect. PD investigates the drug binding to the biological receptors while PK is monitored by absorption, distribution, metabolism, and excretion (ADME) studies. PK and PD are integrated to study the drug dose-drug concentrationresponse relationship. In some cases, a drug candidate or its metabolites can accumulate in tissue which can result in significant toxicity ${ }^{140}$. Toxicology studies the adverse effects of a potential drug by determining the right dosing route (i.e. oral, intravenous (IV), inhalation or dermal) and the dosage (duration/concentration of exposure to drug candidate). Therefore, not only PKPD studies but also toxicology studies of a drug candidate and/or metabolites play a key role in deciding whether a potential drug will get a go or no-go ${ }^{141}$. Throughout the drug discovery and 
development, failing ADME studies are the major reason for a no-go. This occurs not only in early development stages but also in Phase II and Phase III of clinical trials. Compounds that are promising during cell-based assays might not give promising results in vivo due to the complex relationships between human bodies, environmental factors, or even drug characteristics. ADME and toxicology studies are applied at a much earlier stage of the drug discovery and development pipeline to understand and avoid these failing potential drug compounds. What is vital in this process is the ability to measure the distribution and absolute concentration over time of both the drug and corresponding metabolites in tissue samples.

Currently, the measurement of spatial information of pharmaceutical compounds is achieved by combining a whole-body autoradiography (WBA) and an MSI strategy on tissue samples. WBA combines localization information, with spatial resolutions of around $100 \mu \mathrm{m}$, and quantification in one experiment, but it cannot distinguish between drugs and their metabolites. This methodology uses a radiolabel attached to the drug, which is often expensive and time-consuming to prepare, preliminary to dosing. Absolute concentrations are obtained by quantitative WBA and LC-MS/MS on tissue homogenate samples. Despite the fact that LC-MS/MS platforms offer robust and reproducible absolute concentrations, this approach alone is not as it does not provide the spatial information of the drug candidate and its metabolites necessary for pharmacokinetics and toxicology studies. MSI, WBA and LC-MS/MS are often complementarily applied to tissue sections to cover the demand for quantification, spatial distribution of drugs, and identifying corresponding metabolites.

MSI has evolved as a valuable qualitative tissue imaging tool in pharmaceutical research. Multiple reviews have been written about the role of MSI in the pharmaceutical industry $80,83,141,142$. Table 1.1 provides an extensive overview of drugs and metabolites studied qualitatively by MSI which is updated and extended from Swales et al. ${ }^{142}$. The overview provides not only information on the dosing route and concentration but also the tool(s) used for MSI analysis. 


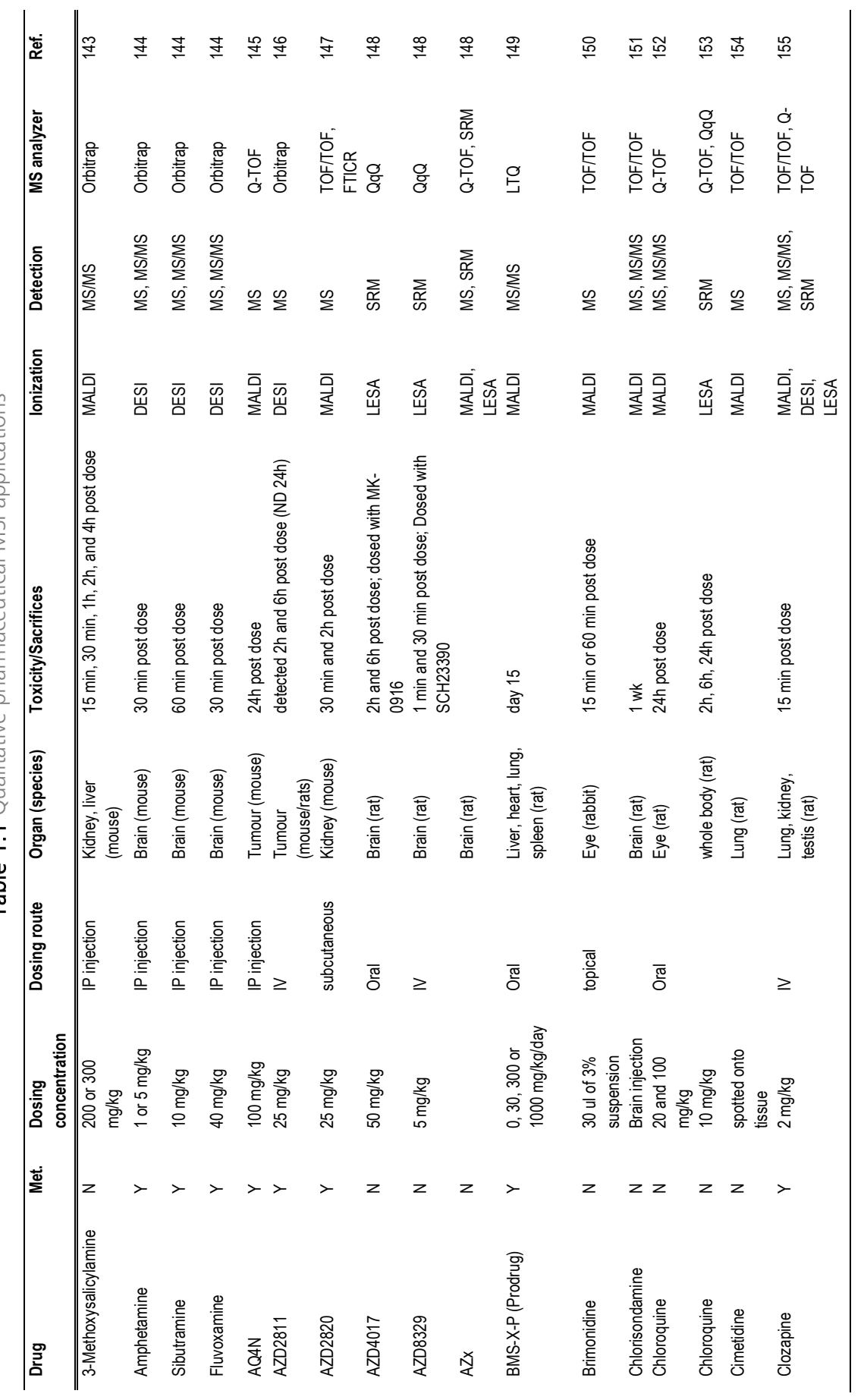




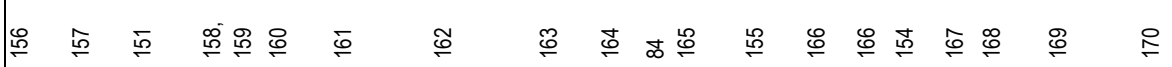

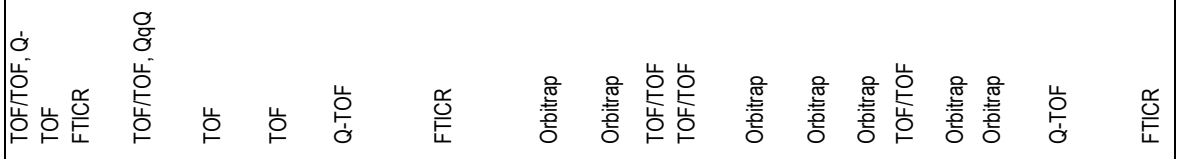

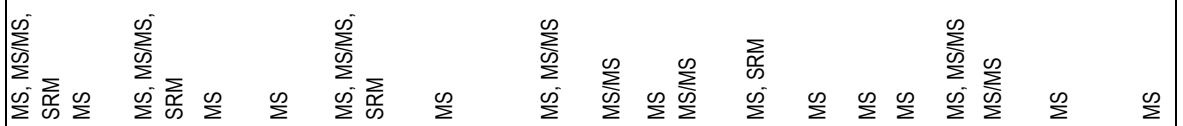

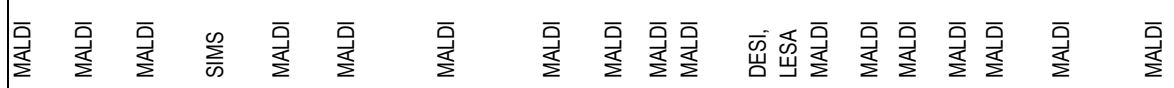

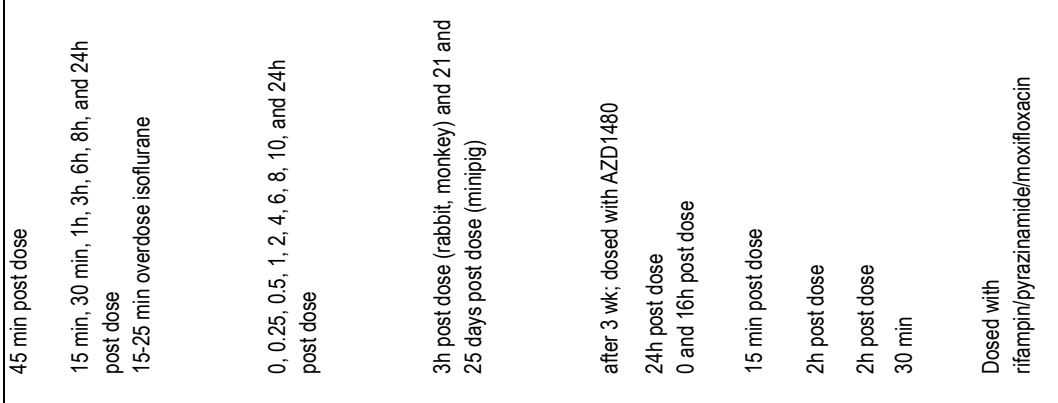

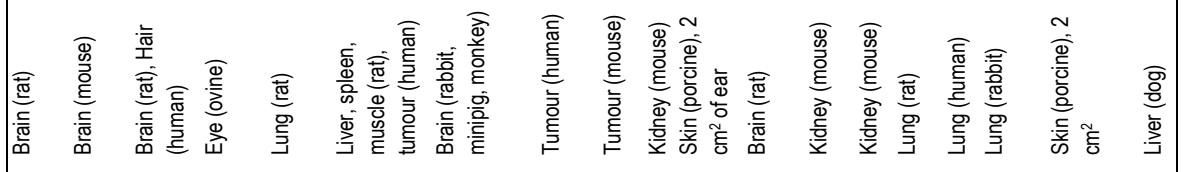

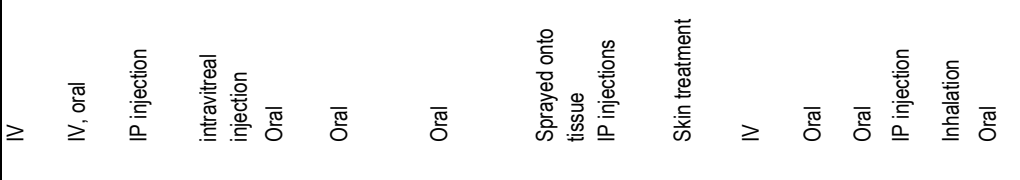

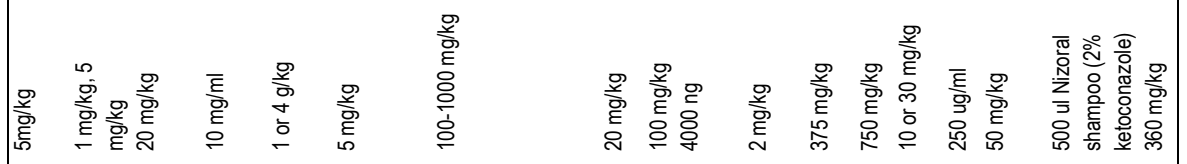
$z z z z z>z z z z z z z z z \quad>>$

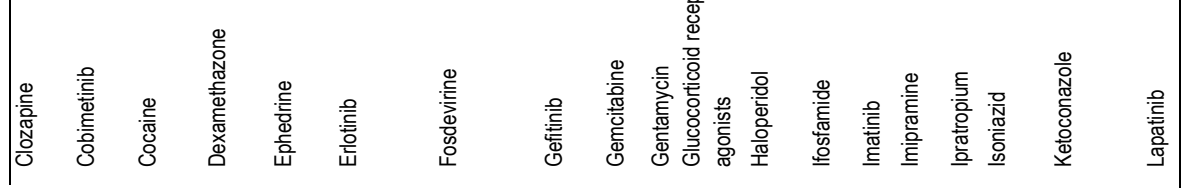




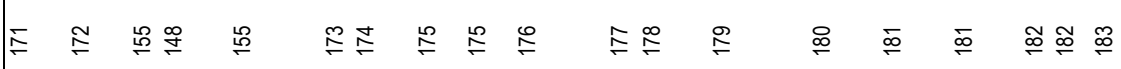

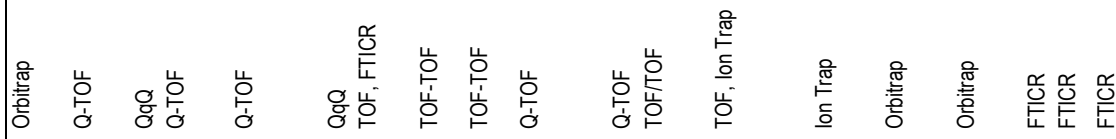

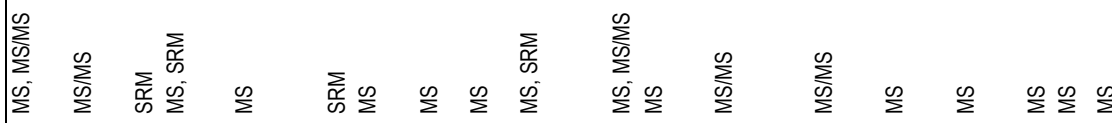

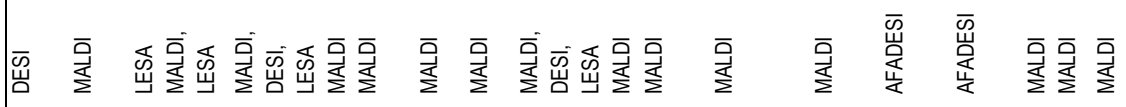

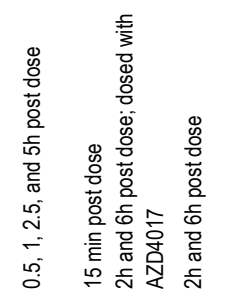

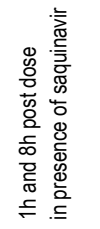

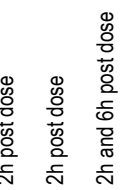

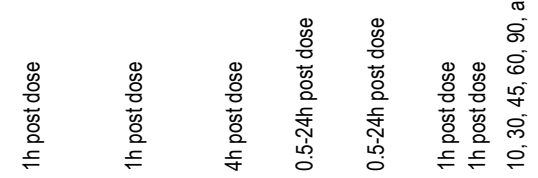

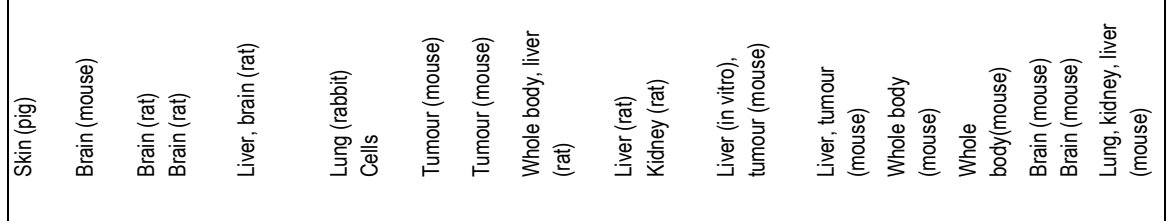
产

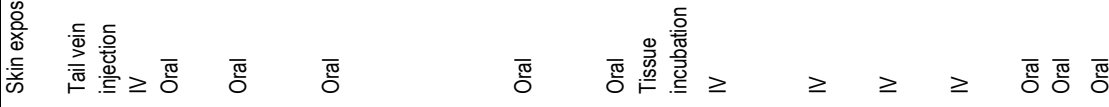

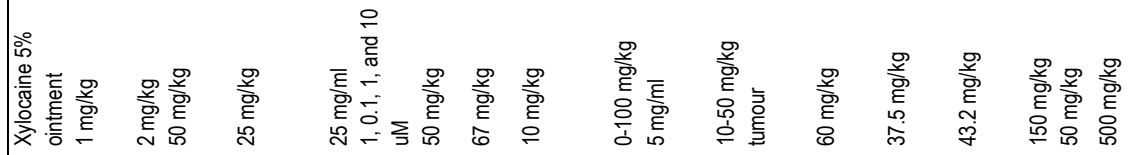

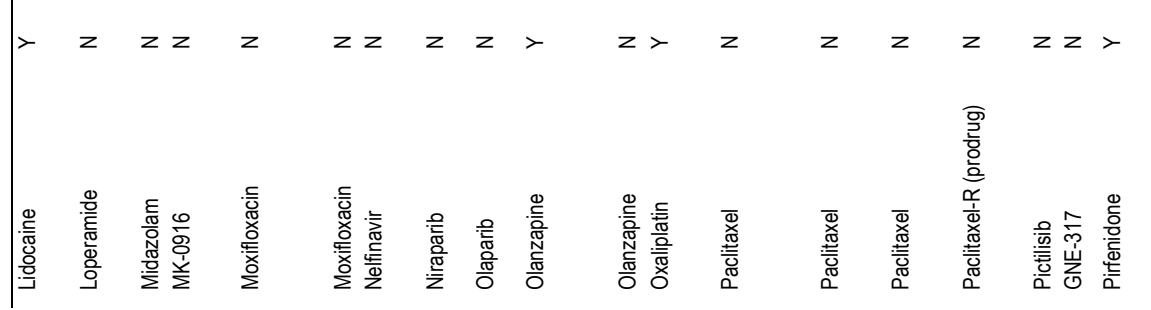


志

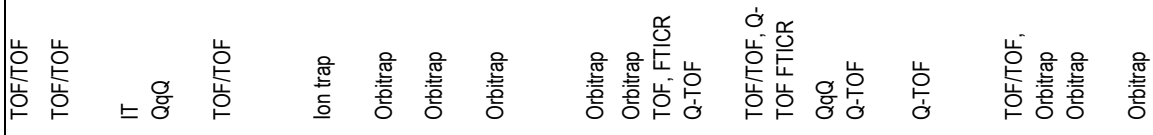

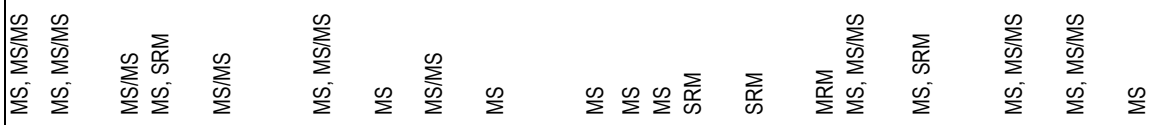

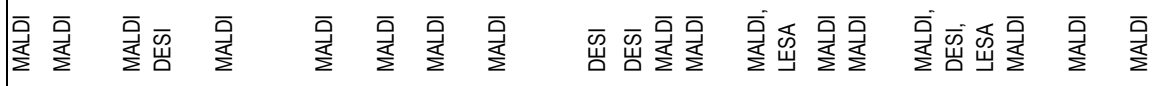

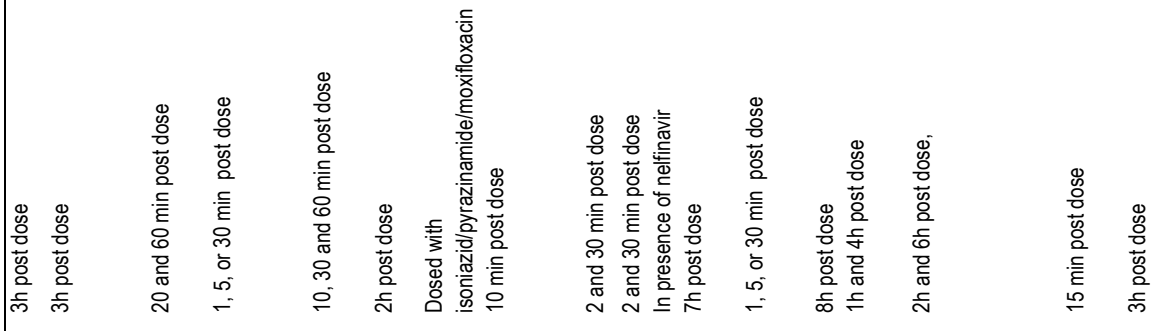

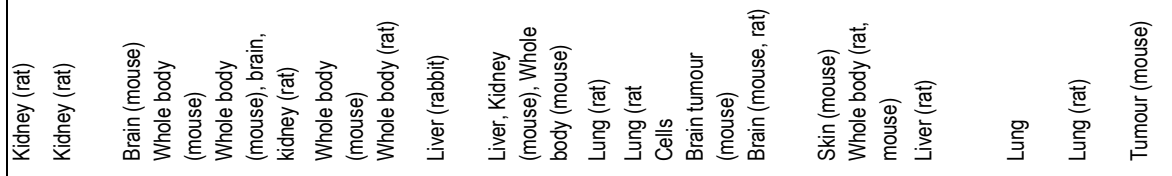

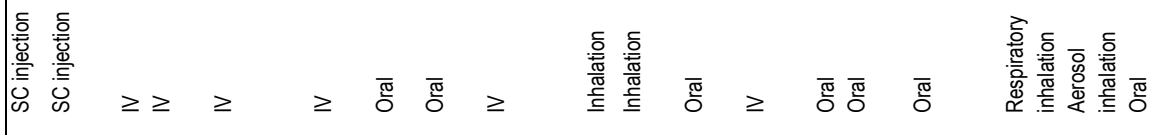

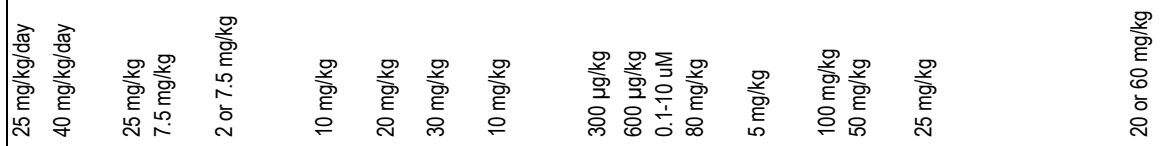

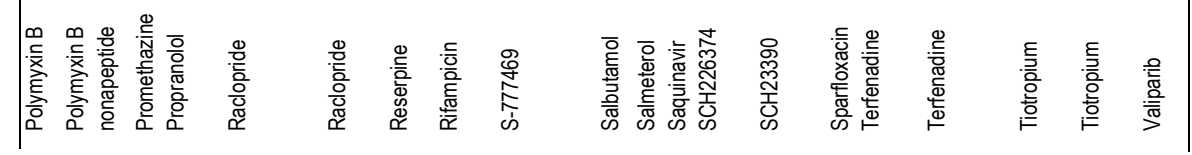


음

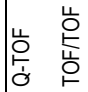

$\sum^{\infty}$

$\sum_{\sum}^{\infty}$

高

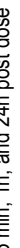

竞

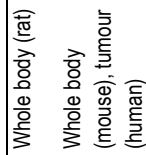

$\geq \geq$

ه

z z

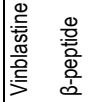


A large number of drug compounds has been studied by MSI, either existing drug compounds or compounds in development. For some of these compounds, metabolites were measured in the tissue section. Dosing concentrations were adapted to the weight of the animal. In most studies, a single drug compound is exclusively investigated. However, a few of the applications explored dosing multiple drug compounds. Swales et al. performed two cassette dosing experiments with different existing drug compounds: i) haloperidol, bufuralol, midazolam and clozapine and ii) moxifloxacin, olanzapine, erlotinib and terfenadine ${ }^{155}$. Two other applications by Manier et al. and Chumbley et al. report MSI analysis of tissues from tuberculosis-infected rabbits which were dosed rifampin, isoniazid, pyrazinamide and moxifloxacin ${ }^{168,} 190$. Tissues analyzed in the applications presented in Table 1.1 ranged from one or several organs to whole body imaging. The majority of the MSI drug applications has been performed by MALDI imaging since it has proven a valuable ionization tool for drug and drug metabolite imaging with high spatial resolution. Since the identification of the drug compounds imaged is obligatory, either MS/MS or SRM/MRM were used to confirm the compounds detected, often in combination with high mass resolving power instrumentation.

\subsection{Quantitative MSI}

The demand for quantitative MSI (Q-MSI) has increased significantly over the years and is currently one of the main challenges for the MSI community. In particular for the pharmaceutical industry, since spatial absolute concentrations while maintaining separation between drug candidate and metabolites is key in the development pipeline and minimizing costly failing drug candidates. The spatial absolute concentrations are used to predict the dose concentration, to perform pharmacokinetic investigations and to measure the toxicity thresholds. In MSI, the lack of sample cleanup causes a relatively large effect of ion suppression on the analyte caused by the surrounding molecular composition. In particular, the heterogeneity in tissue samples increases this ion suppression effect and variability throughout the imaging analysis ${ }^{201,202}$. Quantification in LC-MS/MS often involves the use of internal standards, isotope-labelled analogs of the analyte, to normalize for ion suppression. However, the application of these internal standards and, therefore, gaining reliable quantitative data in MSI still poses practical challenges. Several strategies have been addressed by Ellis et al. and Porta et al. on the implementation of internal standards in MS ${ }^{201}{ }^{203}$. Since the main role of internal standard is to undergo similar ion suppression effects as the analyte of interest, the importance lies in the extraction of the internal standard by the matrix from the tissue sample prior to ionization which is better obtained by applying the internal standard below the tissue sample rather than on top of the tissue sample ${ }^{203}$, 204. Pirman and coworkers used a quantitative MALDI-MSI approach to determine cocaine in brain tissue 
with the use of a deuterated internal standard, as well as the calibration standards, below the tissue sample ${ }^{205}$. The Q-MSI results are compared with the complementary LC-MS/MS and MALDI-MS/MS experiments and show the potential of Q-MSI, however, due to extrapolation to the $x$-axis, the quantitative performance of this approach has still some hurdles to overcome. In addition to spraying the calibration standards below (or on top of) the tissue, tissue homogenates spiked with calibration standards has also proven to provide absolute concentrations which is demonstrated by Groseclose et al. ${ }^{206}$. This time consuming but very reliable approach is called a mimetic tissue model and has been revised to improve the sample preparation ${ }^{207}$.

The use of an isotope-labelled internal standard for absolute quantification is the ideal approach but not always available if a drug candidate is in early stage development. Since ion suppression on the analyte is not only compound specific but also tissue specific, Hamm et al. reported the tissue extinction coefficient (TEC) as a correction factor for different tissue types within a whole body ${ }^{208}$. A known compound is mixed with the MALDI matrix and homogeneously sprayed over the whole body prior to MSI analysis. The TEC is calculated for each tissue organ by dividing the average intensity of the known compound on tissue by the average intensity on the glass slide.

\subsection{Scope and outline}

Challenges in quantitative MSI (Q-MSI) of pharmaceutical compounds are often the result of limited separation prior to MS analysis. Not only does this lead to low analyte coverage due to ion suppression from surrounding sample molecules but also to isobaric interferences in the mass spectrum because of the presence of those surrounding molecules. The aim of this thesis is to develop and apply enhanced separation techniques in the field of ambient Q-MSI. First, novel technological developments are presented to minimize ion suppression and add selectivity and sensitivity. This is executed by the integration of LC, IMS and MRM in an MSI workflow. After the technological development, the right imaging tool is used for the ambient Q-MSI analysis of pharmaceuticals.

In chapter 2, we report an LC-MS setup in combination with high resolution spatial sampling using LESA. This fully automated platform motivates the use of LC and IMS for additional separation to MS. The integration of LC minimizes the inefficient ionization by decreasing the amount of surrounding molecules. IMS separates isobaric background molecules from the analyte and adds selectivity to the high resolution LESA$\mu \mathrm{LC}-\mathrm{IMS}_{\mathrm{M}} \mathrm{MS}^{\mathrm{E}}$ platform. This research is complemented with data-independent analysis for structural identification of peptides. From an application point-of-view, this research 
shows improved neuropeptide coverage in the pituitary gland in an Alzheimer's Disease model.

Chapter 3 presents a Girard's T (GT) derivatization strategy to improve ionization efficiency of poorly ionizable prostanoid metabolites. Separation of these biologically relevant isomers often requires chromatography prior to ionization and MS. For imaging purposes, these GT derivatized prostanoids are used in an IMS comparison for isomeric separation: cyclic IMS, TIMS, and high resolution FAIMS. Does the improved IMS resolution come with a decrease in sensitivity? To answer this question, their biological applicability of these IMS types is shown after an extraction and GT derivatization of endogenous prostanoid metabolites from intestine tissue.

Chapter 4 describes targeted MSI which combines DESI ionization with specific MRM imaging by using a QqQ instrument. All modes of the QqQ instrument are presented for visualization of lipid distributions in brain tissue. High throughput imaging by multiple MRM transitions of the same analyte confirms its spatial localization in a second application, dog liver tissue from a drug distribution study. Dual polarity MRM analysis shows its strength by mapping possible co-localized metabolites and lipids without the need for consecutive tissue sections.

The application of DESI-MRM imaging in Q-MSI is reported in chapter 5. This chapter starts with the presentation of an adapted mimetic tissue model using a 3D printed mold for gelatin embedding. A multiplatform comparison is performed to study the analytical performance of different mass spectrometers (each in different modes) in relationship to MRM imaging. The latter is used for quantitative analysis of different regions of interest in four dog liver tissues from the previous drug distribution study. The Q-MSI data is compared with LC-MS concentration obtained from the same dog livers.

The technological improvements for ambient Q-MSI that are established in this thesis are discussed in Chapter 6: Enhanced analyte coverage, improved isobaric and isomeric separation, higher selectivity and sensitivity, and MRM based quantification. 


$$
2
$$




\section{High Resolution LESA- $\mu L C-I M S-M S^{E}$}

Based on

Integration of Ion Mobility MSE after Fully Automated, Online, High-Resolution Liquid Extraction Surface Analysis Micro-Liquid Chromatography

Lieke Lamont, Mark Baumert, Nina Ogrinc, Mark Allen, Rob Vreeken, Ron M.A. Heeren and Tiffany Porta Analytical Chemistry 201789 (20), 11143-11150 
Direct analysis by mass spectrometry (imaging) has become increasingly deployed in pre-clinical and clinical research due to its rapid an accurate readouts. However, when it comes to biomarker discovery or histopathological diagnostics, more sensitive and indepth profiling from localized areas is required. We developed a comprehensive, fully automated online platform for high-resolution liquid extraction surface analysis (HRLESA) followed by micro-liquid chromatography (LC) separation and a dataindependent acquisition strategy for untargeted and low abundant analyte identification directly from tissue sections. Applied to tissue sections of rat pituitary, the platform demonstrated improved spatial resolution, allowing sample areas as small as $400 \mu \mathrm{m}$ to be studied, a major advantage over conventional LESA. The platform integrates an on-line buffer exchange and washing step for removal of salts and other endogenous contamination that originates from local tissue extraction. Our carry overfree platform showed high reproducibility, with an inter-extraction variability below $30 \%$. Another strength of the platform is the additional selectivity provided by a postsampling gas-phase ion mobility separation. This allowed distinguishing co-eluted isobaric compounds without requiring additional separation time. Furthermore, we identified untargeted and low-abundance analytes, including neuropeptides deriving from the pro-opiomelanocortin (POMC) precursor protein and localized a specific area of the pituitary gland (i.e., adenohypophysis) known to secrete neuropeptides and other small metabolites related to development, growth, and metabolism. This platform can thus be applied for the in-depth study of small samples of complex tissues with histologic features of $\sim 400 \mu \mathrm{m}$ or more, including potential neuropeptide markers involved in many diseases such as neurodegenerative diseases, obesity, bulimia, and anorexia nervosa. 


\subsection{Introduction}

Mass spectrometry imaging (MSI) is being used more often in pre-clinical and clinical research due to its many advantages over conventional imaging techniques ${ }^{209-211}$. MSI offers the possibility to correlate distribution maps of multiple molecular species simultaneously with histological and clinical features without labelling. This methodology enables the discovery of potential diagnostic and prognostic markers of diseases in a single experiment. With these unique features, MSI opens new doors for molecular-driven pathology, in various fields of histopathological diagnostics, such as identification and grading of tumors ${ }^{89,212}$. MSI has also proven to be a powerful tool in drug discovery as it gives insights not only on drug and metabolite distribution and site(s) of action, but also about the biology present at the sites of drug localization relating to treatment efficacy ${ }^{213}$.

Matrix-assisted laser desorption ionization (MALDI) is by far the most popular ionization technique used to map the tissue microenvironment. In this way, molecular classification can be accurately established with different tissue types, including tumors, to improve the accuracy of diagnosis and characterize tumor heterogeneity ${ }^{210}$. MALDIMSI allows in situ tissue characterization at different molecular "omics" levelsmetabolomics, lipidomics, peptidomics and proteomics 214 - and has found numerous clinical applications, including oncology 215 , psychiatric and neurodegenerative disorders $216-218$, cardiovascular diseases ${ }^{219}, 220$, ophthalmology 221 , joint and cartilagerelated disorders 222 . Extensive efforts focused on instrument development to improve spatial resolution, throughput, and sensitivity have placed MALDI-MSI in a competitive position for clinical studies where knowledge about the tumor microenvironment is critical and hundreds of samples require analysis $223-225$. One of the drawbacks of MALDIMSI is the need for the application of homogenous layers of MALDI matrix on the tissue surface and the ion suppression resulting from the extraction process. This is in part due to the lack of separation technology in an imaging experiment and can degrade the sensitivity and quality of data generated.

Recently, ambient ionization techniques have proven their suitability to extract relevant information from complex biological matrices ${ }^{226}$. The main benefit of these techniques is the possibility to analyze directly surfaces with relatively less complex and timeconsuming sample preparation compared to MALDI. This considerably reduces sample handling and speeds up the whole analytical workflow. Desorption electrospray ionization (DESI) ${ }^{117}, 227$ is increasing its application in clinical research as it provides relatively fast, sensitive, and complementary analysis to MALDI-MSI ${ }^{144,228-233}$. DESI allows rapid classification of human tumors based on tissue-specific lipid molecular profiling ${ }^{234}$. One of the most promising applications would be to use the information at the time of surgery on resected specimens to guide surgical resections that could improve management of patients ${ }^{235}$. Liquid extraction surface analysis (LESA) based on a liquid 
microjunction (LMJ) surface sampling was introduced in 2008 and has been employed for profiling biological matrices from localized tissue area in various studies, including

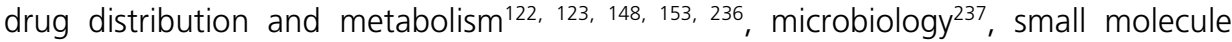
antibody-drug conjugate catabolites ${ }^{238}$, lipidomics ${ }^{236,239}$, proteomics ${ }^{240-242}$, and native mass spectrometry for non-convalent complex studies ${ }^{243}, 244$. Although often criticized for the poor spatial resolution achievable (i.e., $1.2-2.0 \mathrm{~mm}$ with $1 \mu \mathrm{L}$ of solvent deposited on the top of the surface), LESA is an excellent tool for conducting profiling experiments from a selected spot, such as quick metabolite screening or parent drug localization to an organ. Complementary to quantitative whole-body autoradiography that provides distribution information on radiolabeled material, LESA followed by MS detection enables differentiation of a parent drug from its metabolites.

Chromatography-free approaches create ion suppression effects, which enables the identification of analytes of high abundance ${ }^{245}$ but limits protein/peptide identification because of the high degree of cell or tissue complexity. Frequently, compounds are codesorbed and observe interference from structural isomers and/or isobaric compounds (e.g., interfering isotopic clusters). As a result, chromatography-free approaches often suffer from the absence of separation before the mass analysis. Indeed, high resolution is not adequate to distinguish isomers and in some cases tandem mass spectrometry (MS/MS) does not provide enough selectivity. Therefore, additional information and improved selectivity are required to provide adequate identification of biologically relevant analytes. The addition of a post-desorption and post-ionization gas-phase ion mobility spectrometry (IMS) after MALDI, DESI, and LESA has demonstrated to resolve isobaric species and reduce chemical noise ${ }^{122,228,246}$. However, this method does not account for tissue-specific ionization suppression ${ }^{245}$. Because the surface sampling and the ionization processes are resolved both in space and time dimensions, LESA allows for the manipulation of the extracted material in the liquid phase prior to ionization of molecular content ${ }^{247}$. As a consequence, LESA can easily incorporate a liquid-based separation after the surface sampling process, which is not possible with MALDI or DESI ionization techniques. Kertesz et al. developed a LMJ-SS approach followed by HPLCESI-MS for the analysis of drugs and metabolites in whole-body thin tissue sections, which helps to distinguish isomeric phase II metabolites of propanolol ${ }^{123}$. Continuousflow LMJ-SS coupled online with HPLC/MS also enables the extraction, separation, and detection of proteins and low-molecular-weight compounds (e.g., drugs of abuse) from tissue sections and dried blood spots. Usually, the surface of dried blood spots is sufficiently hydrophobic for maintaining a stable liquid junction, even with highaqueous-content solvents ${ }^{135}$. This innovative LMJ-SS-HPLC-MS/MS approach was also used to investigate the distribution of specific markers within normal human pituitary gland and pituitary adenoma tissue sections, to discriminate between tumor and non- 
tumor tissues 248 . However, such targeted approach requires sample cleanup prior to analysis and will not allow broad screening for potential biomarkers.

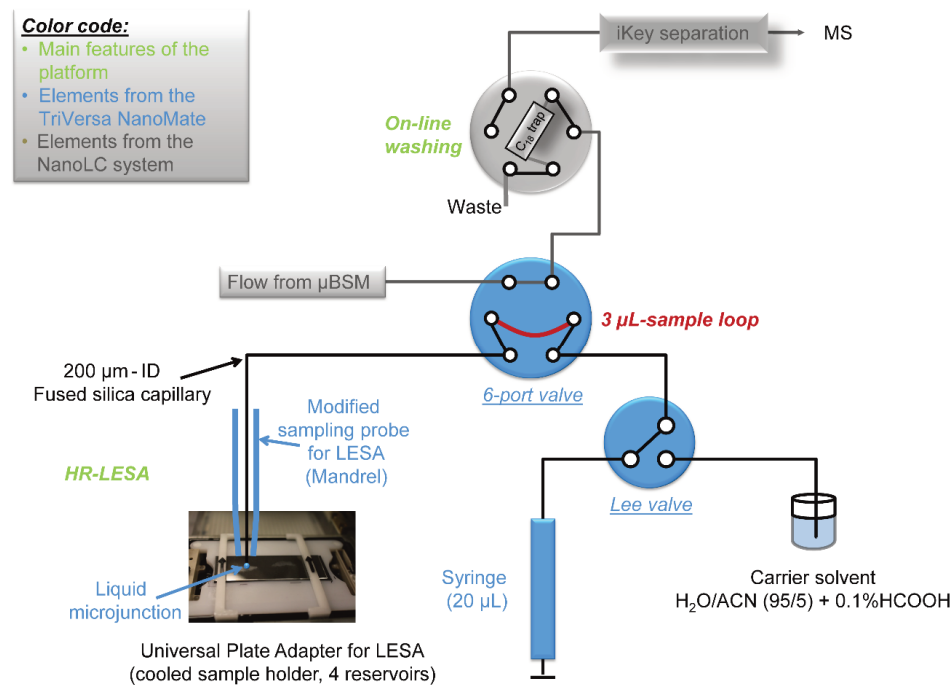

Figure 2.1 Schematic representation of flow connections between the elements of the $\mu \mathrm{LC}$ system (in grey) and the automated sampler device (blue). Main features of the analytical platform are indicated in green. The route of the sample loop is shown in red. The 6-port valve is in the "sample loading" position. HR: high spatial resolution. LESA: liquid extraction surface analysis. BSM: binary solvent manager.

In the present work, we describe a platform developed to improve both poor spatial resolution achieved with "conventional" LESA and ionization suppression effect. The platform was modified for high-resolution (HR)-LESA for direct analysis of endogenous peptides from a $400-\mu m$ area from pre-clinical tissue samples ${ }^{249}$. The HR-LESA was integrated with an online washing step to remove salts and other contaminants, the key source of ion suppression. Micro-LC $(\mu \mathrm{LC})$ was used to separate the analytes of interest from endogenous sample matrix compounds, an additional source of ion suppression. This platform allows isomeric separation due to the implementation of $\mu \mathrm{LC}$ further improved by ion mobility. After this $\mu \mathrm{LC}$ separation, the compounds were analyzed by MS, which combines IMS with MSE, a data-independent acquisition method. Finally, we automated the entire HR-LESA- $\mu$ LC-IMS-MSE platform by implementing new software. This platform is presented here as a complementary addition to the field of mass spectrometry imaging since it opens doors to a more indepth profiling of spatial extractions of biological tissues. 


\subsection{Experimental section}

\section{Chemicals and reagents}

ULC/MS-grade water, ULC/MS-grade acetonitrile (ACN), and 99\% formic acid were purchased from Biosolve (Valkenswaard, NL). Microscopic glass slides were purchased from Thermo Scientific (Braunschweig, DE). Leucine enkephalin standard was provided by a Waters Q-TOF Qualification Standards Kit (Etten-Leur, NL) and prepared at a concentration of $5 \mathrm{ng} / \mathrm{\mu l}$ in ACN/water (50/50; v/v) and used as lock mass.

\section{Murine tissue sectioning}

Healthy Wistar Han rat pituitary gland tissue samples were provided by the Department of General Surgery of the Maastricht University Medical Center (MUMC+). The freshfrozen wild type and transgenic APP ${ }_{\text {KM670/671NL/PS1 }}$ L166P $_{\text {mice }}$ were supplied by the BioImaging Lab, University of Antwerp. Tissues were cryo-sectioned (Microtome cryostat Thermo Scientific, Braunschweig, DE) into 12- $\mu$ m-thick tissue sections and subsequently thaw-mounted on regular microscope glass slides. These tissue sections were stored at $-80{ }^{\circ} \mathrm{C}$ prior to analysis. Right before the analysis, samples were thawed at room temperature and desiccated for $30 \mathrm{~min}$. Hematoxylin and eosin (H\&E) staining was performed on these tissue samples after HR-LESA- $\mu$ LC-IMS-MS analysis.

\section{H\&E staining protocol}

H\&E staining was performed on the same sections used for HR-LESA- $\mu L C-I M S-M S E$ experiments. After MSI analysis, the residual matrix was gently removed by dipping the glass slides in ethanol (EtOH) for 2 minutes. Sections were then washed in successive bathes (96\% EtOH, 70\% EtOH and deionized water - 3 min each). The hematoxylin (Merck, Darmstadt, Germany) staining was then performed for 3 min, slides were then washed in gently running tap water for 3 min, followed by eosin staining for $30 \mathrm{~s}$, washing under running tap water for $1 \mathrm{~min}$ and finally immersed in $100 \% \mathrm{EtOH}$ for 2 min. Slides were finally dehydrated in Xylene (30 s), covered in Etallen and coverslipped. The optical images were acquired using a MIRAX Desk scanner (Zeiss, Gottingen, Germany). Images were acquired with a magnification of 40 .

\section{Conventional LESA extraction}

The LESA extraction was performed using the automated TriVersa NanoMate Advion robot (Advion, Ithaca, NY). A $0.5-\mu \mathrm{L}$ volume of the extraction solvent (ACN/water/formic acid, 70/30/0.1 v/v/v) was deposited with a conductive pipette tip onto the tissue section for $5 \mathrm{~s}$ repeated 1 times. This extract was directly infused into the mass spectrometer (Waters Synapt G2-Si, USA) using chip-based nano-ESI, by applying a nitrogen gas pressure of 0.3 bar and voltage of $1.40 \mathrm{kV}$. 


\section{HR-LESA extraction, online washing, and $\mu \mathrm{LC}$ separation}

High spatial resolution extraction was performed using the capillary extraction arm usually used for coupling with LC-MS fraction collection. Extraction with this setup was performed with $0.5 \mu \mathrm{L}$ extraction solvent (ACN/water/formic acid, 70/30/0.1 v/v/v) after $5 \mathrm{~s}$ in contact with the tissue section. An online and automated buffer exchange was then performed, by diluting 10 times the sample with the carrier solvent (ACN/water/formic acid, 5/95/0.1 v/v/v) - to ensure compatibility with reversed-phase chromatography - and collected in a 3- $\mu \mathrm{L}$ loop (Figure 2.1). Under these conditions, the local extraction was achieved at a spatial resolution of $400 \mu \mathrm{m}$, and the total workflow from extraction solvent aspiration to filling in the sample loop takes $\sim 3 \mathrm{~min}$. Following this extraction, the sample was trapped onto a trap column (ACQUITY UPLC M-class Symmetry $(18,100 \AA, 5 \mu \mathrm{m}, 300 \mu \mathrm{m} \times 50 \mathrm{~mm}$, Waters, USA) and washed with the carrier solvent for $2 \mathrm{~min}$ to remove salts and other possible interferences/contamination. After the 2-min online washing, the trap column was back-flushed onto the $\mu \mathrm{LC}$ lonKey column (iKey BEH C18 Separation Device, $130 \AA$, $1.7 \mu \mathrm{m}, 150 \mu \mathrm{m} \times 50 \mathrm{~mm}$, Waters, Milford, MA) for chromatographic separation as follow: a 13-min gradient from $1 \%$ to $85 \%$ solvent B (ACN/formic acid, 100/0.1) was used for elution of peptides. The column was washed for $3 \mathrm{~min}$ at $85 \%$ solvent B prior to the column equilibration at $1 \%$ solvent B for $5 \mathrm{~min}$. The trap column was equilibrated at $1 \%$ solvent B for $4 \mathrm{~min}$.

\section{Mass spectrometry}

All MS experiments were conducted on a Waters Synapt G2-Si system operated in positive ionization mode (in sensitivity mode). General operating parameters were: capillary voltage $=4 \mathrm{kV}$; source temperature $=80^{\circ} \mathrm{C}$; sampling cone voltage $=40 \mathrm{~V}$; and a desolvation temperature $=150^{\circ} \mathrm{C}$. The default collision energy was set at $4 \mathrm{eV}$ in full MS scan mode. IMS was performed using nitrogen as a drift gas at a flow rate of $90 \mathrm{~mL} / \mathrm{min}$. The TRAP DC entrance was set to $0 \mathrm{~V}$ and the wave height was set to 40 $V$. The velocity of the IMS wave was used to separate the ions over the total $200 \mathrm{~ms}$. The start velocity was set at $1200 \mathrm{~m} / \mathrm{s}$ and the end velocity at $400 \mathrm{~m} / \mathrm{s}$. Dataindependent IMS-MSE acquisition was conducted for the analysis of the pituitary gland and further identification of extracted endogenous peptides. These measurements were performed in the TRANSFER T-wave using a collision energy ramp from 20 to $45 \mathrm{eV}$. The detector voltage was set at $2500 \mathrm{~V}$, and data was acquired within a mass range of $m / z 50-2000$.

\section{Software}

The LESA extraction was controlled by a beta version of the LESA Plus software (Advion, UK), and MassLynx 1.4 (Waters, USA) was used for controlling the online washing step 
and the $\mu \mathrm{LC}$ separation. Data were processed and visualized using Mass Lynx 1.4 (Waters, USA) and DriftScope V2.5 (Waters, USA). The identification of the neuropeptides and proteins was performed using Progenesis QI for proteomics V2.0.5556.29015 (Non Linear Dynamics, USA). For this identification, a species-specific FASTA file was created, and non-specific digest reagent was selected. The amount of missed cleavages was set at 3 and the post-translational modifications (PTMs) allowed in this MSE search were $\mathrm{N}$-acetylation, M-oxidation, and C-carbamidomethylation.

\subsection{Results and discussion}

Here, we describe the HR-LESA- $\mu$ LC-IMS-MS platform and its performance for spatial analysis of neuropeptides. For this purpose, two animal models were used. The first experiments rely in the investigation of neuropeptides in rat pituitary gland tissues, which consists of two different regions with different biological functionalities: the adenohypophysis (anterior lobe and intermediate lobe) and the neurohypophysis (posterior lobe). Therefore, due to the morphology of the tissue $(\sim 2 \times 3 \mathrm{~mm})$, local extraction at high spatial resolution is crucial for molecular characterization of both regions. The second set of experiments is performed on wild type and transgenic mice expressing amyloidosis to assess the potential of the platform to study neuropeptides potentially involved in Alzheimer's disease progression. In this case, the sampling areas include regions with high expression of amyloidosis, such as the cerebral cortex, hippocampus and cerebellum, which also require precise sampling.

\section{Analytical platform}

Spatial resolution. In a conventional LESA set-up, the extraction is achieved using a conductive pipet tip - which, depending on the solvent composition, can lead to a large sampled area up to $2 \mathrm{~mm}^{130}$. This is potentially a limiting factor when analyzing small objects such as the anatomical features of the pituitary gland or any other organs that require precise sampling. In our HR-LESA- $\mu$ LC-IMS-MS platform (Figure 1), we modified the extraction system to improve the spatial resolution and reduce the size of the extracted areas. By using a silica capillary instead, we were able to significantly improve the spatial resolution to $400 \mu \mathrm{m}$ - as assessed under microscopic evaluation of the tissue section after the liquid extraction took place (Figure 2). With an extracted area of 400 $\mu \mathrm{m}$, the molecular content from the anterior and posterior lobe of the pituitary gland can be accurately extracted. We illustrate the ability to unambiguously distinguish between the adenohypophysis and neurohypophysis of the pituitary in Figure 2. Equal spot sizes were observed from the mouse brain tissue section in the H\&E image after HR-LESA- $\mu L C-I M S-M S$. 


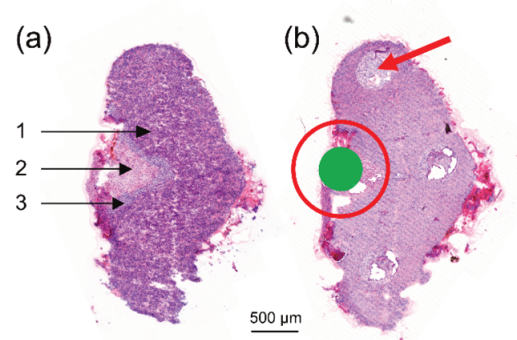

Figure 2.2 H\&E stained images of the pituitary gland before (a) and after (b) HR-LESA extraction show an improvement in spatial resolution with HR-LESA (i.e. $400 \mu \mathrm{m}$ area represented by a green dot in b) compared to conventional LESA (area shows the spot size of a typical LESA extraction (i.e., $1000 \mu \mathrm{m}$-diameter, red circle in b)). In (b), the arrow indicates the trace after an extraction with the capillary. Anatomical features of the pituitary gland containing the adenohypophysis and the neurohypophysis. Anatomical features: 1. Anterior lobe; 2. Posterior lobe; 3. Intermediate lobe.

Reproducibility of the extraction. To investigate the extraction reproducibility and sample carry-over of the HR-LESA setup, extraction of a leucine enkephalin standard was performed followed by flow injection analysis. A 5-ng leucine enkephalin solution (prepared in $\mathrm{ACN} / \mathrm{H}_{2} \mathrm{O} ; 50 / 50 ; \mathrm{v} / \mathrm{v}$ ) was spotted onto a hydrophobic plate and air-dried. The extractions of 5 consecutive spots using $0.9 \mu \mathrm{L}$ of $50 \% \mathrm{ACN}+0.1 \% \mathrm{HCOOH}$ and the modified HR-LESA extraction system resulted in a coefficient of variation of $30 \%$ (based on the surface area from the extracted ion chromatogram at $\mathrm{m} / \mathrm{z} 556.28$ (protonated species [LeuEnk $+\mathrm{H}]^{+}$) for each extracted spots; $n=5$; Figure 2.3).

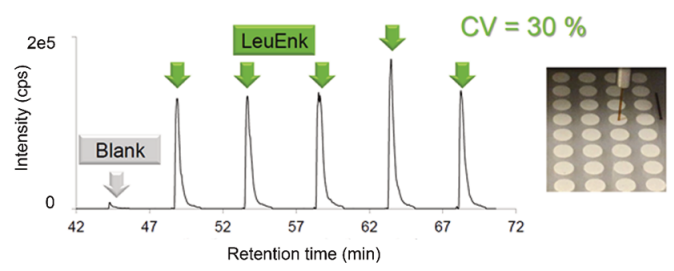

Figure 2.3 Evaluation of the reproducibility of the extraction with the silica capillary probe followed by FIA. Results display XIC at m/z 556.28 (protonated Leucine-Enkephalin ion [LeuEnk $+\mathrm{H}]^{+}$) for one blank (glass slide) followed by 5 extractions of LeuEnk using $0.9 \mu \mathrm{L}$ of $50 \% \mathrm{ACN}+0.1 \% \mathrm{HCOOH}$. Photograph displays the silica capillary probe during the extraction from the plate. CV based on absolute area $(n=5)$.

Integration of online clean up between extraction and chromatographic separation. The tissue sample was placed in the sample plate holder as shown in the photograph in Figure 2.1. This tissue extract was then collected in a 384-well plate and diluted with carrier solvent. The diluted extract was 'injected' by the same fused silica capillary, collected in the 3- $\mu \mathrm{L}$ loop, and, after switching the 6-port valve, trapped onto the $\mathrm{C}_{18}$ trap column for an online washing with water/ACN (99/1; v/v) to remove all water- 
soluble matrix compounds. The trap column was back-flushed to separate the remaining sample on an lonkey separation device using a reversed phase $\mu \mathrm{LC}$ separation.

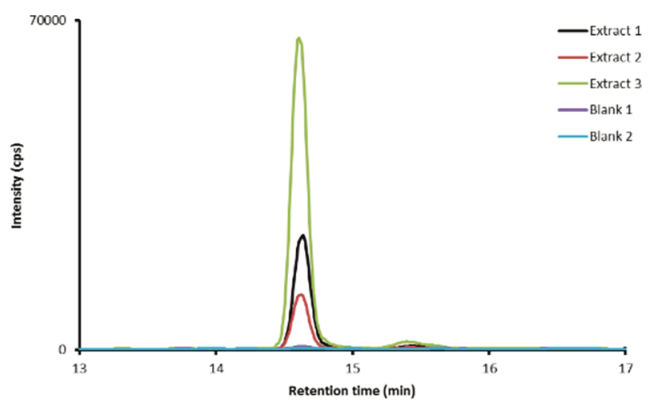

Figure 2.4 Reproducibility of LESA- $\mu$ LC-MS platform using leucine enkephalin standard spotted onto hydrophobic plate. Three extracts were taken, followed by two blank extracts. The relative standard deviation of the retention time of the leucine enkephalin extraction is $<0.12 \%(n=3)$. The carry-over in the first blank extraction is $<1.1 \%$ considering the absolute peak area of the last leucine enkephalin extraction versus the absolute peak area of the first blank extraction.

Reproducibility of the chromatographic separation and sample carry-over. HR-LESA combined with the online washing and $\mu \mathrm{LC}$ separation lonKey system was evaluated. The chromatographic peak of leucine enkephalin was found at a retention time of 16 $\mathrm{min}$. This extraction was performed in triplicate to test the retention time reproducibility followed by two blank extractions to study carry-over. The relative standard deviation of the retention time was $<0.12 \%$ and the carry-over in the first blank extraction was $<1.1 \%$ using the absolute peak area, which demonstrates a good reproducibility of chromatographic separation (Figure 2.4). The minimal occurrence of sample carry-over was due to the online clean-up of the sample loop during the analysis. In addition to the leucine enkephalin standard extraction, reproducibility of the chromatographic separation of neuropeptides from mouse brain and corresponding carry-over has been investigated (Figure 2.5a and 2.5b). Relative standard deviation values vary from $7.1 \%$ to $25.4 \%$ and the sample carry-over is $<0.8 \%$ (Figure $2.5 c-2.5 f$ and 2.6 ). These values were obtained from three tissue extractions followed by a blank extraction in use of the absolute chromatographic peak area. While the implementation of $\mu \mathrm{LC}$ into our platform significantly minimizes the amount of ionization suppression, the reproducibility could be considerably improved by the addition of an isotope-labelled internal standard to the tissue sample. 

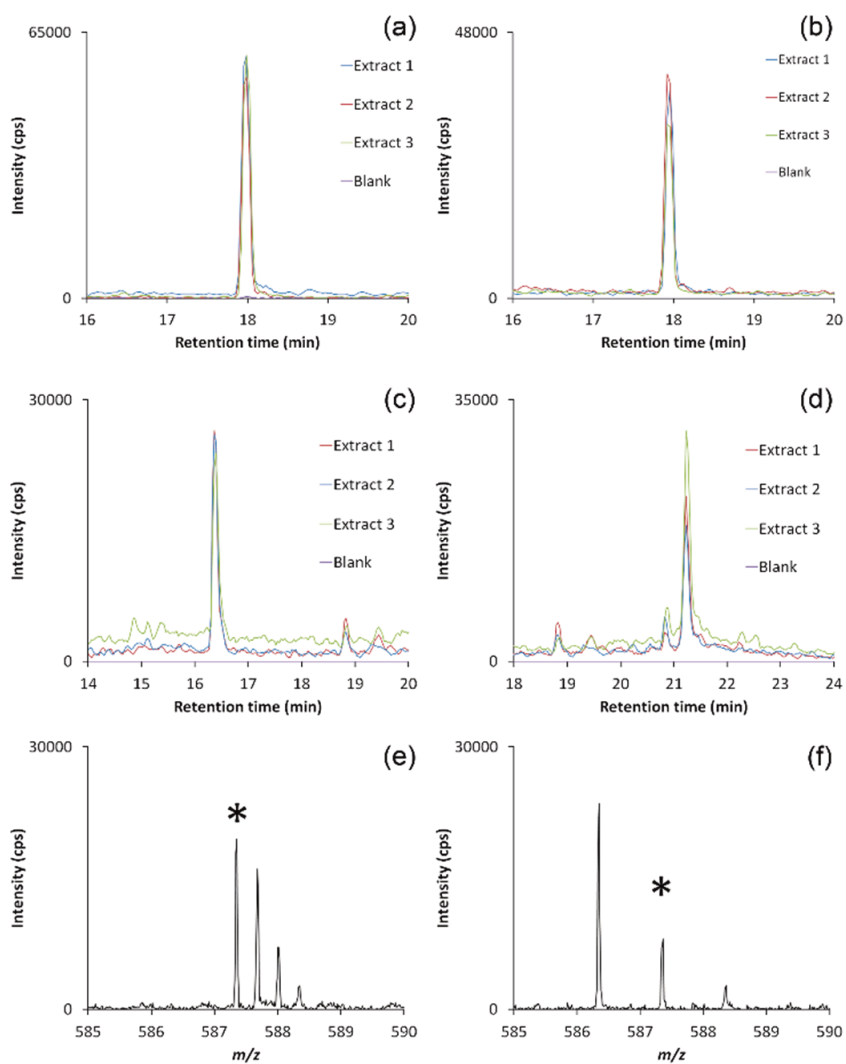

(e)

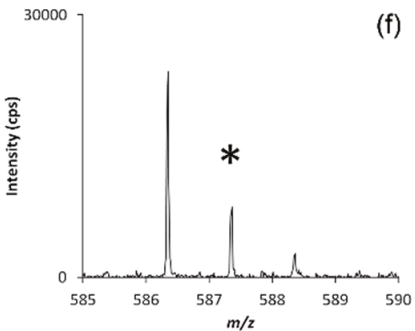

Figure 2.5 Reproducibility and isobaric separation of HR-LESA- HLC-IMS-MSE platform using APP and WT mouse brain tissues. Three extracts from each tissue section were taken from the cerebral cortex region, followed by one blank extract from the glass slide. For the reproducibility test, XIC are shown of $m / z 634.38$ at $6.02 \mathrm{~ms}$ arrival time from APP brain (a) and WT brain (b). The relative standard deviation for the three extractions $(n=3)$ considering the peak areas are $7.1 \%(a)$ and $13.3 \%(b)$. For the isobaric separation, the XIC of two neuropeptides are shown of $\mathrm{m} / \mathrm{z} 587.35$ from APP brain with corresponding mass spectrum at retention time $16.36 \mathrm{~min}$ with $5.33 \mathrm{~ms}$ arrival time (c) and $21.23 \mathrm{~min}$ with $8.17 \mathrm{~ms}$ arrival time (d). The relative standard deviation of the three extractions $(n=3)$ at retention time $16.36 \mathrm{~min}$ and $21.23 \mathrm{~min}$ are $9.3 \%$ (c) and $25.4 \%(d)$, respectively. Carry-over was assessed by dividing the absolute peak area of the last brain extraction (green) by the absolute peak area of the following blank extraction (purple). Carry-over values were: $0.6 \%(\mathrm{a}), 0.7 \%(\mathrm{~b}), 0.8 \%(\mathrm{c})$ and $0.7 \%(\mathrm{~d})$. The asterisk $\left(^{*}\right)$ corresponds to $\mathrm{m} / \mathrm{z}$ used to extract the ion chromatogram (XIC). 


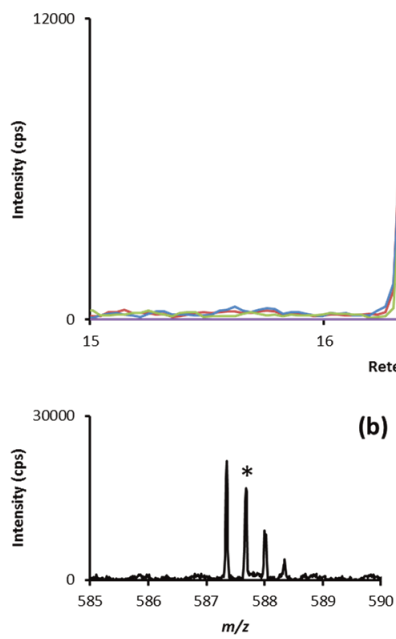

(a)

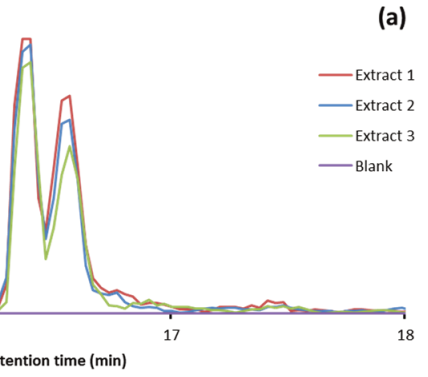

(b)

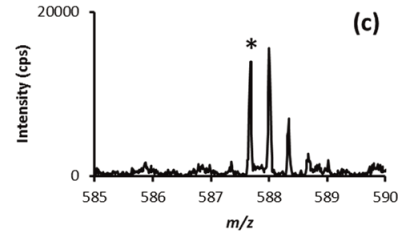

Figure 2.6 Isobaric separation of neuropeptides in the cerebral cortex region of APP mouse brain. Three extractions were performed from the tissue section, followed by one blank extract from the glass slide. XIC are shown of $\mathrm{m} / \mathrm{z} 587.35$ from APP brain (a) with corresponding mass spectrum at retention time $16.36 \mathrm{~min}$ (b) and $16.57 \mathrm{~min}$ (c) both at $5.26 \mathrm{~ms}$ arrival time. The relative standard deviation of the three extractions $(n=3)$ at retention time $16.36 \mathrm{~min}$ and $16.57 \mathrm{~min}$ are $9.0 \%$ and $14.4 \%$, respectively. Carry-over was assessed by dividing the absolute peak area of the last brain extraction (green) by the absolute peak area of the following blank extraction (purple). Carry-over values were: $0.5 \%$ (at $16.36 \mathrm{~min}$ ) and $0.1 \%$ (at $16.57 \mathrm{~min}$ ). The asterisk $\left(^{*}\right)$ corresponds to $\mathrm{m} / \mathrm{z}$ used to extract the ion chromatogram (XIC).

\section{Post-surface sampling chromatographic separation}

Having demonstrated that the HR-LESA provided higher spatial resolution, good reproducibility, and minimal sample carry-over, we implemented a $\mu \mathrm{LC}$ separation to enable isomeric separation and further sample cleanup. Isomers have the same mass and the same molecular formula, in contrast to isobaric compounds, which have the same nominal mass but differ in molecular formula. MS alone is insufficient to separate and accurately identify isomeric compounds (even with high mass resolution instrumentation). Furthermore, ion suppression often occurs and is considered the primary cause of irreproducibility in MS. Because LC is a powerful tool for separation of these isomeric compounds ${ }^{130}$, we integrated an online washing step and $\mu \mathrm{LC}$ separation after the surface sampling and prior to electrospray ionization (Figure 2.1). Compared to conventional LESA, the HR-LESA approach with online washing and $\mu \mathrm{LC}$ separation showed an increase in sensitivity and selectivity for leucine enkephalin in the adenohypophysis region of rat pituitary online (Figure 2.7). When we performed our HR-LESA- $\mu$ LC-IMS-MSE approach for the analysis of pituitary, 67 compounds could be chemically identified while only 5 and 4 compounds could be found in the conventional LESA-IMS-MS and LESA-MS data, respectively ${ }^{137}$. For the mouse brain cerebral cortex 
analysis, 14 compounds could be identified with HR-LESA- $\mu$ LC-IMS-MSE while only 3 and 2 compounds were observed in the conventional LESA-IMS-MS and LESA-MS data, respectively. In view of the even larger spot size of LESA-MS, the minimization of ionization suppression by $\mu \mathrm{LC}$ is crucial for detection of lower abundant compounds. Although the implementation of $\mu \mathrm{LC}$ in our approach requires a longer analysis time (30 min/spot) compared to conventional LESA-MS (1 min/spot), this can be justified due to the enhanced detection limits of the HR-LESA- $\mu$ LC-IMS-MSE platform compared to conventional LESA-IMS-MS and LESA-MS.

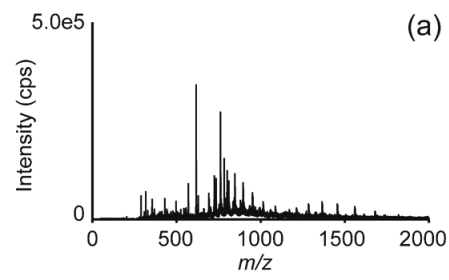

(a)
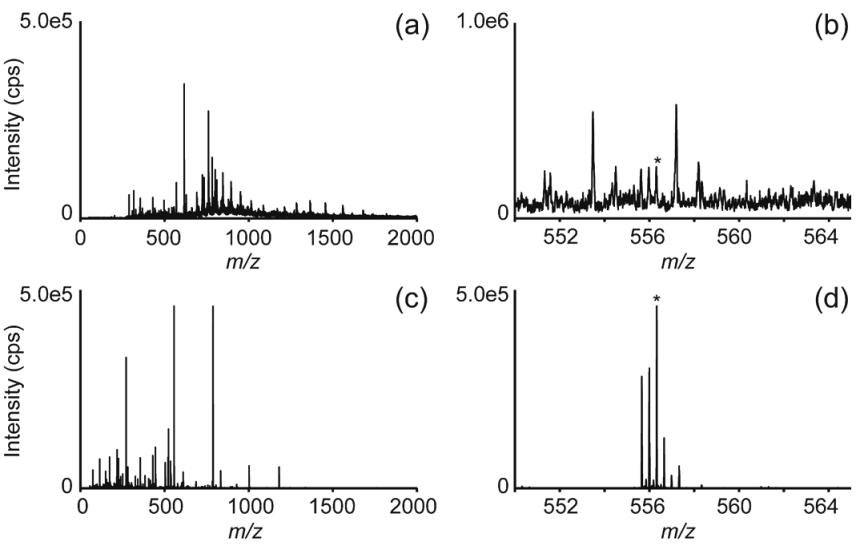

(c)

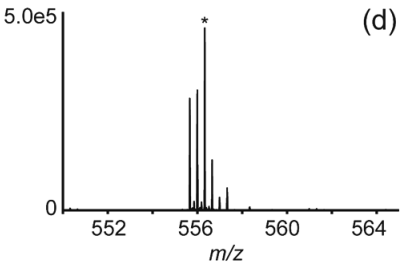

Figure 2.7 Sensitivity of LESA-MS versus HR-LESA- $\mu$ LC-MS: The total mass spectrum (a and c) and a zoomedin mass spectrum ( $b$ and $d$ ) of the adenohypophysis region of rat pituitary gland using LESA-MS ( $a$ and $b$ ) compared to HR-LESA- $\mu$ LC-MS (c and d). The zoomed mass range displays a low-intensity peak corresponding to the protonated molecule of leucine enkephalin (marked with *), which is known to be present in this region. This is extracted from the ion chromatogram at retention time at 16 min. The zoomed mass range from HR-LESA- $\mu L C-M S$ shows the presence of leucine enkephalin co-eluting with a triply charged species, which was not observed with LESA-MS.

Interestingly, in the pituitary data, we observed the presence of a triply charged species (Figure 2.7d) co-eluting with the leucine enkephalin, which was not detected with the conventional LESA extraction (Figure 2.7b). This difference can be explained by the occurrence of ion suppression, which was minimized by HR-LESA-HLC-MS. The extracted ion chromatogram $(\mathrm{XIC})$ based on the protonated molecule signal of leucine enkephalin $(m / z 556.28 \pm 0.05)$ extracted from the adenohypophysis region showed a retention time of $16 \mathrm{~min}$, similar to that of the monoisotopic peak of the triply charged species $(m / z 555.65$, Figure $2.7 a)$.

However, the corresponding mass spectrum shows a different isotope pattern belonging to a triply charged species (Figure 2.8b, green asterisk). This clearly exposes the limitations of LC separation and need for additional separation power to increase 
selectivity and accurate identification. For this reason, an additional gas-phase IMS was added to the analytical workflow to increase the analytical content of the data.
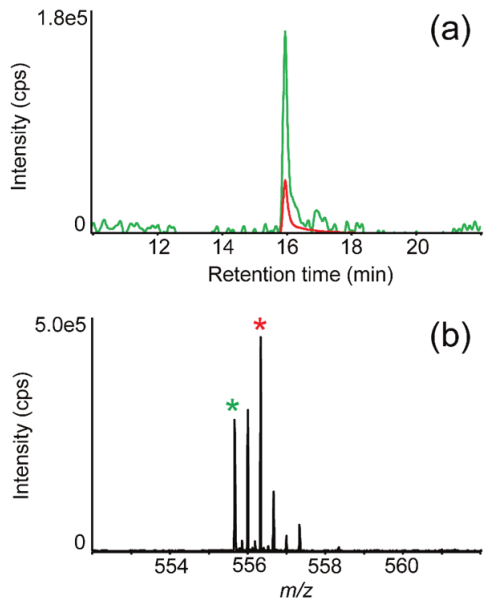

Figure 2.8 The extraction of two co-eluting compounds: the XIC (a) and corresponding mass spectra (b). The green XIC represents the chromatogram extracted from the monoisotopic peak of the triply charged species at $m / z 555$.65. The red XIC represents the chromatogram extracted from leucine enkephalin at $m / z 556.28$.

\section{Ion mobility spectrometry (IMS) of isobaric species}

We employed tri-wave ion mobility to achieve additional separation: the TRAP T-Wave region was used for trapping and accumulating ions, IMS T-Wave region for subsequent separation, and the TRANSFER T-Wave region to focus the ions and as fragmentation cell when performing data-independent IMS-MSE measurements. IMS was performed from the anterior lobe HR-LESA extraction $\left(\mathrm{m} / \mathrm{L} 556.28\right.$ eluting at $t_{R}=16 \mathrm{~min}$; Figure 2.9a). The extracted ion mobility arrival time spectrum (Figure 2.9b) depicts two arrival time peaks, which can be labelled using the corresponding mass spectra as leucine enkephalin (Figure 2.9d) and its co-eluting compound (Figure 2.9c). The mass spectra extracted at this specific retention time displayed clean signals with overall improved sensitivity. The observed isotope pattern of the triply charged species has a mass of 1664 Da after deconvolution. The advantage of the integration of IMS after chromatographic separation is the additional separation power that is gained to separate co-eluting isomeric species, without compromising the overall analysis time. For the identification of this co-eluting species and other extracted proteins, IMS-MSE analysis was performed. IMS-MSE is a data-independent acquisition (DIA) mode that includes both high and low collision energy measurements alternated in one parallel analysis using the retention time to match precursor and fragment ions. Species can be 
identified in one analysis without requiring additional data-dependent MS/MS experiments by performing IMS-MSE analysis.
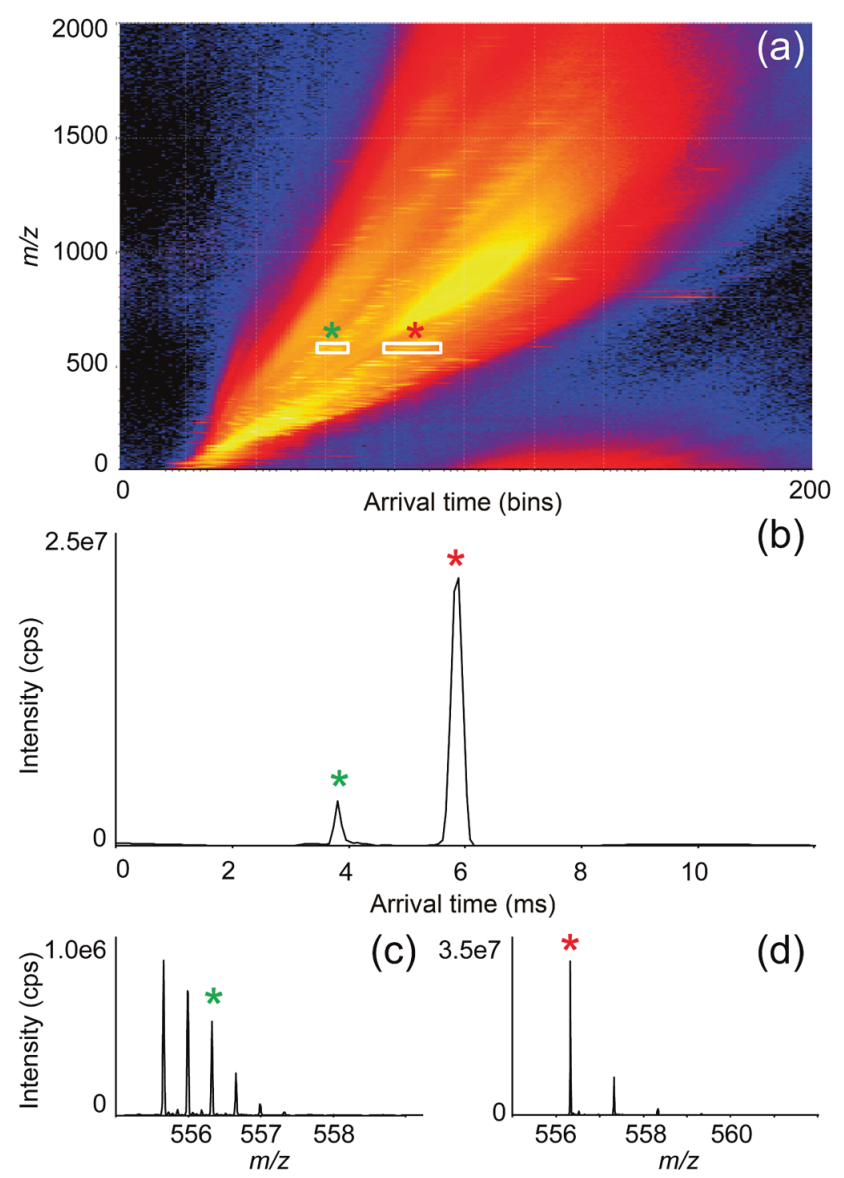

Figure 2.8 The ion mobilogram (a) of the extract from the anterior lobe of pituitary gland is depicted. The extracted ion mobility arrival time spectrum (b) shows the separation of two co-eluting compounds. The extracted mass spectra at arrival time $3.8 \mathrm{~ms}$ (c) and $5.6 \mathrm{~ms}$ (d) show the separation of both compounds, indicated in red (leucine encephalin) and green ( $\mathrm{N}$-acetylated alpha-melanocyte stimulating hormone).

\section{Identification of neuropeptides and proteins present in the pituitary gland}

We sought to identify neuropeptides and proteins in the rat pituitary samples using our platform by integrating data-independent acquisition (DIA). We chose the DIA approach because data-dependent acquisition (DDA) often neglects low-abundance precursor ions, which limits the discovery of untargeted analytes and markers ${ }^{250}$. One DIA approach, MSE, collects TOF mass spectra with and without fragmentation by 
alternating the energy of the collision cell between low and high values ${ }^{250}$. Another approach, sequential window acquisition of all theoretical fragment-ion spectra (SWATH), was developed on hybrid Q-TOF mass analyzers that offer resolving power of $20,000-40,000^{251}$. Both approaches were demonstrated to be particularly powerful in detecting low-abundance analytes for further metabolite and peptide identifications. We integrated IMS-MSE acquisition into our platform.

IMS-MSE detects both precursor ions and fragments of the precursor ions fragmented independently on their abundance ${ }^{252}$. Progenesis QI for proteomics software uses an algorithm that also performs a database search considering retention time, mass accuracy, and PTMs ${ }^{250}$. However, due to the DIA nature of these analyses, this algorithm is based on physicochemical properties of the peptides and proteins. These characteristics are used to calculate the correlation to models with regards to hydrophobicity and gas-phase separation and are, therefore, applicable to reversedphase LC and IMS. In similar strategy to DDA to calculate false-positive identification rates, we applied decoy and species-specific databases. Sensitivity and selectivity are significantly increased because of the repetitive approach of this algorithm. After the identification of the most abundant protein, this data is removed from the database, and another search is executed to identify the second most abundant protein. After removing the data from the second most abundant protein, a third search is performed. This process is continued until all proteins are identified. 


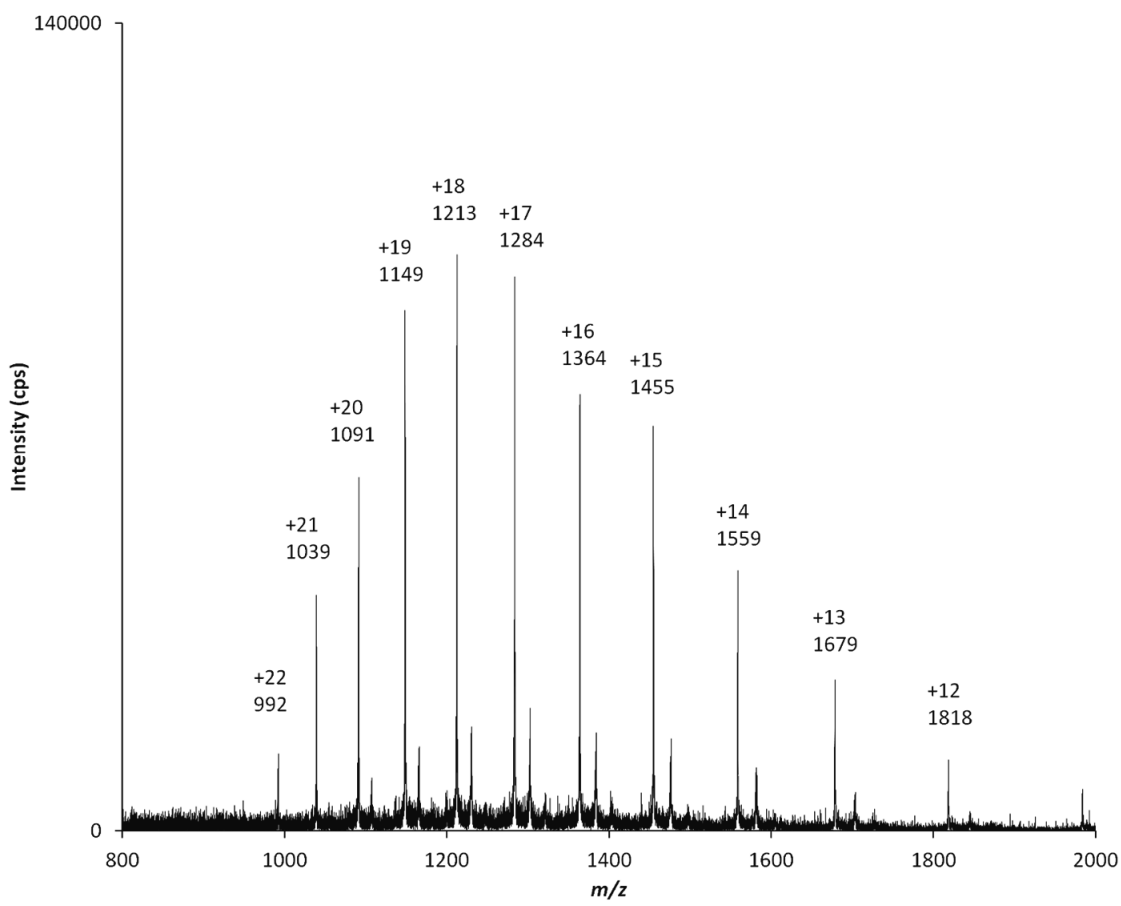

Figure 2.10 Intact protein extracted from the adenohypophysis region of the pituitary gland. Deconvolution of the mass spectrum allowed determining the mass of an intact protein at $21832 \mathrm{Da}$. This protein was identified as POMC.

Table 2.1 Pituitary gland proteins identified from HR-LESA- $\mu$ LC-IMS-MSE analysis

\begin{tabular}{lllll}
\hline $\begin{array}{l}\text { Accession } \\
\text { number }\end{array}$ & $\begin{array}{l}\text { Peptide } \\
\text { count }\end{array}$ & $\begin{array}{l}\text { Unique } \\
\text { peptides }\end{array}$ & $\begin{array}{l}\text { Confidence } \\
\text { score }\end{array}$ & Description \\
\hline \hline Q7TNJ2 & 1 & 1 & 2.7696 & ATP-binding cassette sub-family A member 7 \\
P01186 & 17 & 17 & 155.3358 & Vasopressin-neurophysin 2-copeptin \\
P01194 & 25 & 25 & 183.6023 & Pro-opiomelanocortin \\
\hline
\end{tabular}


Table 2.2 Mouse brain proteins identified from HR-LESA- $\mu$ LC-IMS-MSE analysis

\begin{tabular}{|c|c|c|c|c|}
\hline $\begin{array}{l}\text { Accession } \\
\text { number }\end{array}$ & $\begin{array}{l}\text { Peptide } \\
\text { count }\end{array}$ & $\begin{array}{l}\text { Unique } \\
\text { peptides }\end{array}$ & $\begin{array}{l}\text { Confidence } \\
\text { score }\end{array}$ & Description \\
\hline Q2TA51 & 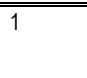 & 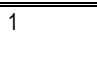 & 3.90 & $\begin{array}{l}\text { Keratin-associated protein } 5-5 \text { OS=Mus musculus } G N=K r t a p 5-5 P E=2 \\
S V=1\end{array}$ \\
\hline P43277 & 3 & 2 & 8.71 & Histone H1.3 OS=Mus musculus GN=Hist1h1d PE=1 SV=2 \\
\hline Q64507 & 1 & 1 & 3.31 & $\begin{array}{l}\text { Keratin-associated protein } 5-1 \text { OS=Mus musculus } G N=K r t a p 5-1 \quad P E=2 \\
S V=1\end{array}$ \\
\hline Q8VI56 & 1 & 1 & 2.08 & $\begin{array}{l}\text { Low-density lipoprotein receptor-related protein } 4 \text { OS=Mus musculus } \\
G N=L r p 4 P E=1 S V=3\end{array}$ \\
\hline P43275 & 1 & 1 & 3.34 & Histone H1.1 OS=Mus musculus GN=Hist1h1a PE=1 SV=2 \\
\hline Q8C0Q3 & 1 & 1 & 2.76 & $\begin{array}{l}\text { Tetratricopeptide repeat protein } 34 \text { OS=Mus musculus } \mathrm{GN}=\mathrm{Ttc} 34 \mathrm{PE}=2 \\
\mathrm{SV}=2\end{array}$ \\
\hline 055111 & 1 & 1 & 4.30 & Desmoglein-2 OS=Mus musculus GN=Dsg2 PE=1 SV=3 \\
\hline P54320 & 2 & 2 & 8.74 & Elastin OS=Mus musculus $\mathrm{GN}=\mathrm{E} \ln \mathrm{PE}=1 \mathrm{SV}=2$ \\
\hline Q9JHT5 & 1 & 1 & 4.47 & $\begin{array}{l}\text { AMME syndrome candidate gene } 1 \text { protein homolog OS=Mus musculus } \\
\mathrm{GN}=A m m e c r 1 \mathrm{PE}=2 \mathrm{SV}=1\end{array}$ \\
\hline P43276 & 1 & 0 & 4.90 & Histone H1.5 OS=Mus musculus GN=Hist1h1b PE=1 SV=2 \\
\hline
\end{tabular}

In the extracts from the rat pituitary gland, we identified vasopressin and POMC (Figure 2.10), based on 17 and 25 peptides, respectively (Table 2.1) ${ }^{250}$. These peptides, which are deriving from the pro-opiomelanocortin (POMC) precursor protein, are important signaling molecules in regard to feeding behavior and, therefore, involved in diseases like obesity, bulimia, and anorexia nervosa ${ }^{253,254}$. In the mouse brain cerebral cortex extracts, we identified 14 peptides (Table 2.2). After applying de novo peptide sequencing to the fragmentation spectrum of the co-eluting species at $\mathrm{m} / \mathrm{z} 1664$ at the retention time of $16 \mathrm{~min}$, this compound was identified as $\mathrm{N}$-acetylated alphamelanocyte stimulating hormone based on nine fragment ions: $y_{1}, b_{1}, y_{2}, y_{7}^{+2}, y_{5}, y_{10^{+2}}$, $y_{12}{ }^{+2}, y_{7}$, and $y_{8}$, deriving from P01194 (124-136). The MS/MS spectra can be found in Figure 2.11 ( $a$ and b). Based on the $\mathrm{MS}^{\mathrm{E}}$ data, the singly charged species was identified as leucine enkephalin (Figure 2.11c). 

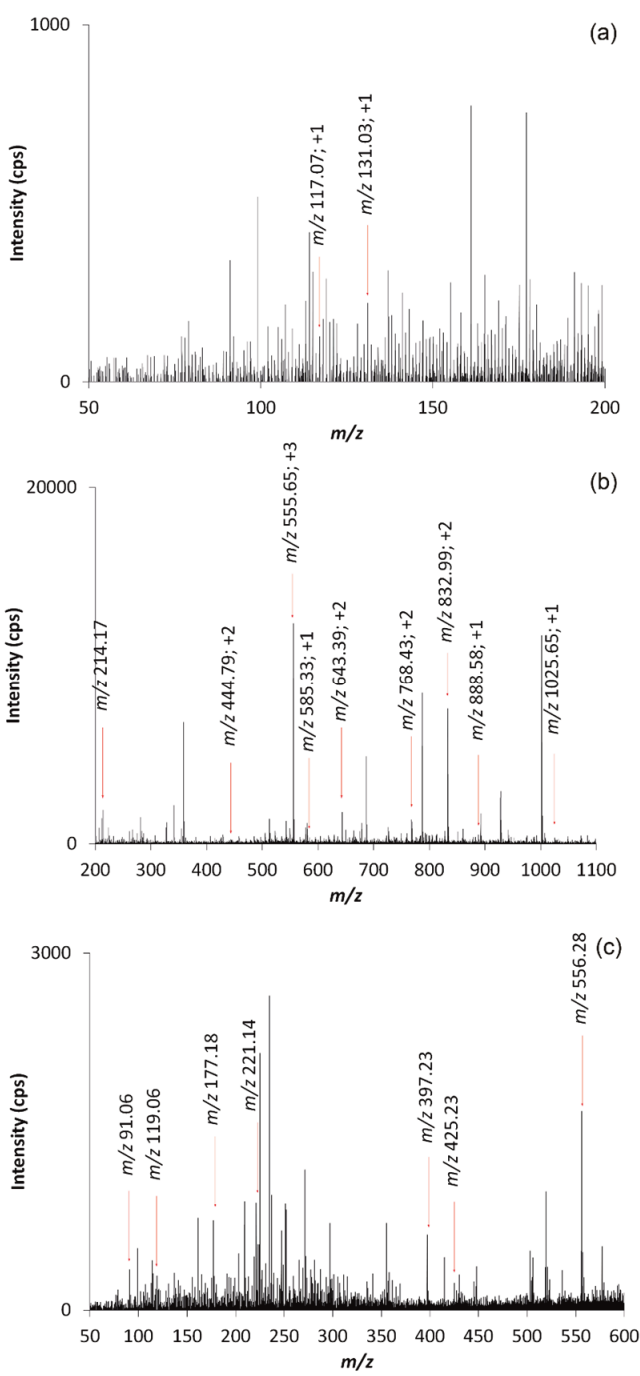

Figure 2.11 MSE data and fragments of alpha-MSH (precursor $\mathrm{m} / 2555.65,+3$ ) extracted from the pituitary gland. Spectra are shown at high (a) and low (b) collision energy after background subtraction. Fragments from the triply charged precursor were identified as follows: $m / z 117.07$ (y1 ion), m/z 131.03 (b1 ion), m/z 214.17 (y2 ion), $m / z 444.79$ (y7 ion; +2 ), $m / z 555.65$ (precursor; +3 ), $m / z 585.33$ (y5 ion), $m / z 643.39$ (y10 ion; +2$), m / z 768.43$ (y12 ion; +2 ), $m / z 832.99$ (precursor; +2$), m / z 888.58$ (y7 ion), and $m / z 1025.65$ (y8 ion). This data correlates with previously published data presented ${ }^{255}$. MS $\mathrm{S}^{\mathrm{E}}$ data and fragments of leucine enkephalin (precursor $m / z 556.28,+1$ ) extracted from the pituitary gland. Spectrum is shown at high collision energy at $6.02 \mathrm{~ms}$ arrival time after background subtraction. Fragments from the triply charged precursor were identified as follows: $\mathrm{m} / \mathrm{z} 91.06, \mathrm{~m} / \mathrm{z} 119.06, \mathrm{~m} / \mathrm{z} 177.18, \mathrm{~m} / \mathrm{z} 221.14$ (b2 ion), $\mathrm{m} / \mathrm{z} 397.23$ (a4 ion) and $\mathrm{m} / 2425.23$ (b4 ion). This data is confirmed with a METLIN database search. 


\subsection{Conclusion}

This work reports the development of an automated and integrated platform combining the advantages of both spatial sampling with LESA and chromatographic separation $(\mu \mathrm{LC})$ for the direct analysis of tissue sections with potential clinical, pre-clinical relevance. In addition, the platform was strengthened by the integration of ion mobility spectrometry (IMS) and data-independent acquisition (DIA) for both the separation and the identification of neuropeptides from a complex tissue extract from a $400 \mu \mathrm{m}$ area. Improvement over conventional LESA of the spatial resolution capabilities of the surface-sampling process was achieved, down to a spatial resolution of $400 \mu \mathrm{m}$, by modifying the sampling probe. The platform demonstrated strong reproducibility, minimal carry-over, increased sensitivity, technical reproducibility, and identification of isobaric compounds.

The advantages of this integrated platform hold strong potential for pre- and clinical applications. Currently, the extraction efficacy is limited to soluble proteins with the number of protein and peptide identifications at fewer than 500. In particular, lowabundance analytes can be identified in complex and small tissue samples, as demonstrated with rat pituitary and mouse brain here. Information about protein isoforms, important in many neurodegenerative diseases, is predicted to be elucidated with the application of our platform with classical in-gel proteomics ${ }^{241}$. This approach paves the way for imaging researchers to increase the total number of proteins and peptides identified and enable regional quantification-based on-tissue analysis. 



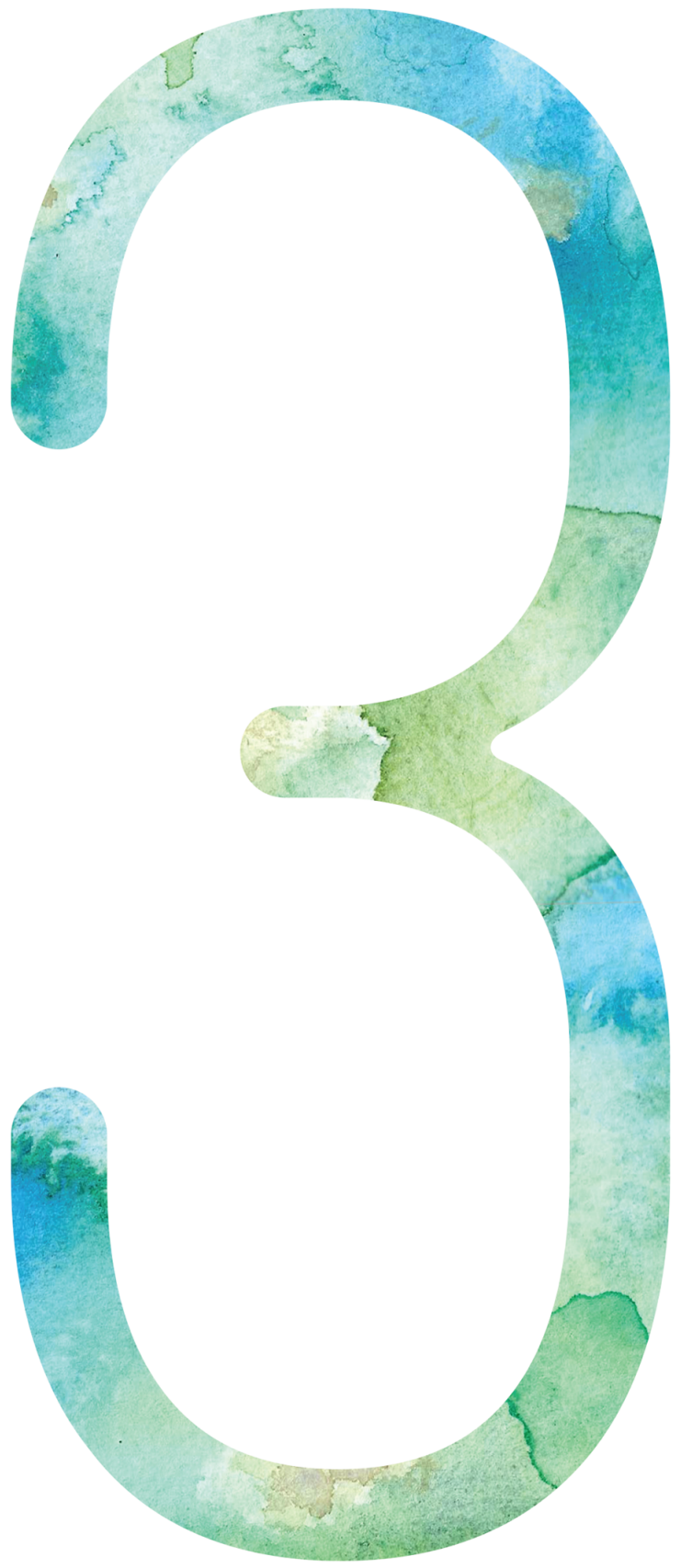




\section{High Resolution IMS-MS for Isomeric Prostanoid Separation}

Based on

High Resolution Ion Mobility Separation Mass Spectrometry for Isomeric Separation of Prostanoids after Derivatization

Lieke Lamont, Andrew P. Bowman, Darya Hadavi, Dale Cooper-Shepherd, Martin Palmer, Bryn Flinders, Jan Jordens, Ynze Mengerink, Maarten Honing, James Langridge, Tiffany Porta Siegel, Rob J. Vreeken and Ron M. A. Heeren

Manuscript in preparation 
High resolution Ion Mobility Spectrometry (IMS) based on linear ion transport in low-tomoderate electric fields and nonlinear ion transport in strong electric fields emerges as a broad approach for rapid separations prior to mass spectrometry (MS). Here we present a comparison of three high resolution IMS techniques: linear cyclic IMS, linear Trapped IMS, and nonlinear high-Field Asymmetric Waveform IMS for the isomeric separation endogenous detection of prostanoids present in intestinal tissue. Inefficiency in negative ionization mode ESI for these compounds (due to low proton affinity of carboxylic group) was overcome by derivatization with Girard's reagent T. Multiple isomers with distinct in vivo function crucial to understand the inflammation process, including the prostaglandins $E_{2}\left(P_{G}\right)$ and $D_{2}\left(P D_{2}\right)$ were included in our IMS comparison. Strengths and weaknesses of both linear and nonlinear IMS were revealed and discussed in which linear IMS provided the best sensitivity and nonlinear IMS the best resolution. This work portends well for high resolution IMS-MS as a powerful platform to disentangle the isomeric complexity in biological systems. 


\subsection{Introduction}

The inflammation process plays a key role during disease development, including arthritis, cardiovascular disease and cancer; and is often at the center of multiple investigations. As a counter reaction to inflammation, an immune response triggers increased amounts of mast cells releasing histamine, tryptases, and eicosanoids, which are implicated in the heat and swelling ${ }^{256,257}$. Eicosanoids, in particular prostanoids, are of interest due to their pro-inflammatory properties and increased presence in inflamed tissue. Prostanoids are products derived from arachidonic acid after its release from the sn-2 position of membrane phospholipids through the action of phospholipase $A_{2}$. Prostaglandin G/H synthases (PGHS), often referred to as cyclooxygenase (COX), further catalyzes the reaction towards the intermediate prostaglandin $\mathrm{H}_{2} 258$, 259. This intermediate prostaglandin is then, depending on the activity of the associated enzyme, converted into one of the active prostanoid end products (Figure 3.1): Prostaglandin $D_{2}$ $\left(\mathrm{PGD}_{2}\right)$, Prostaglandin $\mathrm{E}_{2}\left(\mathrm{PGE}_{2}\right)$, Prostaglandin $\mathrm{F}_{2 \mathrm{a}}\left(\mathrm{PGF}_{2 \mathrm{a}}\right)$, Prostaglandin $\mathrm{I}_{2}\left(\mathrm{PGI}_{2}\right)$, or Thromboxane $\mathrm{A}_{2}\left(\mathrm{TxA}_{2}\right)^{260,261}$.

(a)

(d)<smiles>CCCCCC(O)/C=C/C1C(=O)C[C@@H](O)[C@@H]1C/C=C/CCCC(=O)O</smiles>

(b)<smiles>CCCCCC(O)/C=C/C1[C@H](C/C=C/CCCC(=O)O)C(=O)C[C@H]1O</smiles><smiles>CCCCCC(O)/C=C/C1C2C/C(=C/CCCC(=O)O)OC2C[C@H]1O</smiles>

(c)

(e)<smiles>CCCCCC(O)/C=C/C1[C@H](O)CC(O)[C@@H]1C/C=C/CCCC(=O)O</smiles><smiles>CCCCCC(O)/C=C/C1OC2CC1OC2C/C=C/CCCC(=O)O</smiles>

Figure 3.1 Molecular structures of 5 prostanoid end products of the COX pathway: PGD 2 (a), PGE (b), PGF 2 a (c), $\mathrm{PGI}_{2}(\mathrm{~d})$, and $\mathrm{TXA}_{2}(\mathrm{e})$. 
This COX pathway can be separated into two different pathways, COX-1 and COX-2, due to the existence of different isoforms of the PGHS. The end products of COX-1 and COX-2 have a similar structure but play a significantly different role in the biology. With taking exceptions into account, one could state that in general the COX-1 pathway is responsible for fundamental prostaglandin synthesis while COX-2 is involved in the prostaglandin production due to inflammatory response $\mathrm{e}^{258}$. In bioanalytical studies, this distinction between COX-1 and COX-2 is crucial.

Conventional liquid chromatography tandem mass spectrometry (LC-MS/MS) profiling platforms have been developed for (high-throughput) screening of eicosanoids in biological samples ${ }^{262-264}$. Most of these platforms require time-consuming sample preparation steps and chromatographic separation for isomeric compounds (e.g. PGE 2 and $P \mathrm{PD}_{2}$ ). In addition, the free carboxylic acid groups of the eicosanoids offer very poor ionization efficiency in negative ionization mode ${ }^{265}$. Therefore, derivatization strategies of eicosanoids have been employed successfully to increase ionization efficiency for analysis in positive ionization mode ${ }^{266}$. Some of these derivatization reagents, such as Girard's reagent T, target the carbonyl group which present in prostanoids. The analysis of choice for eicosanoid screening requires efficient ionization, high sensitivity and selectivity in complex biological samples, combined with powerful stereoisomeric separation. The latter is demanded due to the substantial impact of stereoisomerism on the biological activity of eicosanoids. For this reason, chromatography is often chosen for eicosanoid analysis $267,268$.

As an alternative to chromatographic separation approaches, ion mobility spectrometry (IMS) offers a separation technology in which ions are electrically driven through a buffer gas ${ }^{55,269}$. IMS has shown its potential for isomeric separation while removing background from complex biological samples ${ }^{270}$. There are several different IMS technologies available: Drift Tube IMS (DTIMS)271, 272, Traveling Wave IMS (TWIMS) ${ }^{58,273}$, Trapped IMS (TIMS) ${ }^{63,64}$, and high-Field Asymmetric Waveform IMS (FAIMS; also known as differential IMS or DMS) ${ }^{67}$. IMS is already widely used in the field of proteomics ${ }^{52,274}$, lipidomics ${ }^{228}$, and metabolomics ${ }^{275,276}$. Dwivedi and coworkers presented a screening method for a wide range of metabolites (including eicosanoids) using DTIMS ${ }^{275}$. This work proved to separate isobaric eicosanoids but still lacks sufficient ion mobility resolving power for isomeric separation (e.g. $P G E_{2}$ and $\mathrm{PGD}_{2}$ ). Higher ion mobility resolving power is necessary for the separation of structural eicosanoid isomers. From the different types of IMS, three IMS techniques have shown high ion mobility resolving power: cyclic IMS $(R<300)$, TIMS $(R<300)$ and FAIMS $(R<500)^{277}$. Cyclic IMS (cIMS) is based on the TWIMS principle ${ }^{58}$ but prolongs the path of the IMS cell by a loop configuration ${ }^{278}$. Ions can pass this IMS loop multiple times and, therefore, increase their total path length. The amount of passes can be adapted to the separation required. Researchers have recently investigated CIMS for polymer analysis 279 , RNA 
modifications ${ }^{280}$, and carbohydrates ${ }^{281}$. TIMS applies a reversed principle compared to CIMS. TIMS uses a buffer gas to introduce ions to the TIMS cell. These ions are trapped by applying a counteracting electric field while the buffer gas moves in the exit direction ${ }^{282}$. The ion trapping time is $10 \mathrm{~ms}$ up to $1 \mathrm{sec}$ dependent on the separation power necessary ${ }^{64}$. Several TIMS applications have been reported on proteins ${ }^{283}$, peptides $^{284}$, and small molecules ${ }^{285-287}$. FAIMS ${ }^{66,67,288}$ applies an asymmetric waveform (high and low electric fields) across two parallel plates to which gas-phase ions are introduced by a carrier gas ${ }^{277}$. This waveform comprises a short high-field positive and long low-field negative segments that integrate to zero over a period. Since the ion mobility $(K)$ is different at $E_{\text {low }}$ and $E_{\text {high, }}$ the ion will tend towards one of the two plates ${ }^{65}$. A compensation voltage $(\mathrm{CV} ; \mathrm{V})$ is superposed on the waveform to correct the ion's trajectory and to prevent collision with one of the plates (deionization). Alteration of this $\mathrm{CV}$ allows selection of ions, with specific ion mobility differentials, to be transferred to the mass spectrometer. Scanning the $\mathrm{CV}$ is often expressed by a compensation field $\left(E_{c} ; / / c m\right)$ due to different plate distances used between FAIMS devices. FAIMS research has focused on protein analysis ${ }^{289-293}$, though applications can be found for analysis of glycans $^{294}$, lipids ${ }^{246,295,296}$, and metabolites ${ }^{122}$.

Advantages of CIMS, TIMS and FAIMS are not only the isomeric separation power but also its ability to significantly reduce chemical noise from complex samples, which is of particular importance when analyzing low-level endogenous eicosanoids. To our knowledge, only limited research is published using high resolution ion mobility separation for isomeric eicosanoid separation and, if so, it is used in combination with $\mathrm{LC}^{297}$.

Here, we investigated the use of three IMS systems for the analysis of prostanoids in complex biological tissue, namely: linear cIMS, linear TIMS, and nonlinear FAIMS. Girard's reagent T (GT), already used to derivatize both endogenous ${ }^{298-300}$ and exogenous ${ }^{110,} 127$ compounds, was assessed for derivatization of prostanoids which improves ionization efficiency in positive ionization mode and decreases possible ion suppression from the sample matrix. We hypothesize that the GT derivatization can assist in the improvement of ion mobility separation through an increased difference of collisional cross section. In this work, we performed an IMS comparison to test this hypothesis. We subsequently validated our approach by derivatizing mini pig intestine extracts with GT to investigate the endogenous detection of prostanoids.

\subsection{Experimental section}

\section{Materials}

HPLC-grade water and HPLC-grade methanol $(\mathrm{MeOH})$ were purchased from Fischer Chemical (Fair Lawn, NJ, USA). Reagent grade $>95 \%$ formic acid and GT were acquired 
from Sigma-Aldrich (St. Louis, MO, USA). All prostanoid standards were purchased from Cayman Chemical (Ann Arbor, MI, USA) and prepared to a $20 \mu \mathrm{M}$ mixture in $\mathrm{MeOH}$. Identification and FAIMS peak assignment was executed by direct infusion of individual standard at the same concentration (e.g. $20 \mu \mathrm{M}$ in $\mathrm{MeOH}$ ). Mini pig intestine tissue was provided by Janssen Pharmaceutica (Beerse, BE). Titan3, $17 \mathrm{~mm}$ filters with a pore size of $0.45 \mu \mathrm{m}$ were purchased from Thermo Fisher (Rockwood, TN, USA).

\section{Direct infusion-MS}

Prostanoid concentrations used for direct infusion were concentrated to $1 \mu \mathrm{M}$ in a $\mathrm{MeOH} /$ water solution (80/20; $\mathrm{v} / \mathrm{v}$ ). For analysis in positive ionization mode, $0.2 \%$ formic acid and $10 \mathrm{mg} / \mathrm{ml} \mathrm{GT}$ was added to the prostanoid standard mixture. This mixture was incubated at room temperature for 5 minutes. Direct infusion experiments were performed on a high mass resolution (140,000 at $\mathrm{m} / \mathrm{z} 200)$ Q-Exactive ${ }^{\mathrm{TM}}$ orbitrap mass spectrometer equipped with a heated ESI source (HESI-II probe, Thermo Scientific). Negative and positive ions were generated at a $5 \mu \mathrm{l} / \mathrm{min}$ flow rate while applying a capillary voltage of $5.0 \mathrm{kV}$ and $4.0 \mathrm{kV}$, respectively. The mass spectrometer operated in normal MS mode and MS/MS spectra were acquired using collisional induced dissociation (CID) fragmentation. The normalized collision energy applied was in the range of 35-40 arbitrary units depending on the compound fragmented. Data was acquired at a mass range of $m / 250-750$ and recorded using Thermo Xcalibur Roadmap (Thermo Scientific, version 2.8).

\section{Tissue extract preparation}

Frozen mini pig intestine was received from Janssen R\&D (Beerse, BE) and stored at -80 ${ }^{\circ} \mathrm{C}$. Eight, $500 \mu \mathrm{m}$ thick sections were taken from the intestine sample at $-21^{\circ} \mathrm{C}$ using a Microtome cryostat (Thermo Scientific, Braunschweig, DE) and collected in a $1.5 \mathrm{ml}$ Eppendorf tube. For the prostanoid extraction, $500 \mu \mathrm{l} \mathrm{MeOH}$ was added to the tube and 3 repetitive freeze-thaw cycles ( $10 \mathrm{~min}$ on dry-ice followed by 10 min sonication) were executed. After extraction, this sample was centrifuged (Eppendorf, $13000 \mathrm{rcf}, 10$ $\mathrm{min}$ ) to separate the supernatant from the lysate. Supernatant was collected in a clean $1.5 \mathrm{ml}$ Eppendorf tube and stored at $-80^{\circ} \mathrm{C}$. Prior to the analysis, the thawed sample was centrifuged for $5 \mathrm{~min}$ and filtered over a $0.45 \mu \mathrm{m}$ filter to prevent needle clogging during the nanoESI analysis.

\section{High resolution IMS-MS experiments}

Cyclic IMS. Samples were infused at a $3 \mathrm{ul} / \mathrm{min}$ flow rate into a prototype cIMS-MS instrument based on a modified SYNAPT G2-Si instrument (Waters Corporation, UK) after positive Nano-ESI ionization. The cIMS separator consists of a multifunction array of electrodes forming an orthogonal closed loop. The cIMS chamber has a path length 
of $98 \mathrm{~cm}$ with a single pass revolving power of $\sim 65$. The velocity was set to $375 \mathrm{~m} / \mathrm{s}$ and the T-wave height was $25 \mathrm{~V}$. The TOF mass range was set to $\mathrm{m} / \mathrm{z}$ 50-1000. Data was acquired and processed using a custom MassLynx data acquisition with new interactive instrument control software (Waters Corporation, UK).

TIMS. Samples were introduced at a $3 \mathrm{ul} / \mathrm{min}$ flow rate into the TIMS-tof instrument (Bruker Daltonics, USA) and ionized using positive ESI. General ESI parameters were: capillary voltage $3.5 \mathrm{kV}, \mathrm{N}_{2}$ pressure at 0.8 bar (11.6 psi), drying gas flow rate of 4.0 $\mathrm{l} / \mathrm{min}$, and a drying temperature of $200{ }^{\circ} \mathrm{C}$. Voltages were applied at the entrance and exit of the TIMS tunnel, $255 \mathrm{~V}$ and $55 \mathrm{~V}$, respectively. The $t_{\text {ramp }}$ applied was $450 \mathrm{~ms}$. Nitrogen was used as the TIMS drift gas. The TOF mass range was set to $m / z 400-500$. Data acquisition and processing occurred with Compass DataAnalysis v5.0 software (Bruker Daltonics, USA).

FAIMS. NanoESI-FAIMS-MS experiments performed on a custom made FAIMS device ${ }^{65}$ connected to an LTQ $X^{T M}$ linear ion trap mass spectrometer (Thermo Scientific, Waltham, MA, USA) equipped with an ion funnel (Heartland Mobility) ${ }^{301}$. Negative and positive nanoESI occurred by infusion of the sample at a flow rate of $300 \mathrm{nl} / \mathrm{min}$ and a capillary voltage of $3.8 \mathrm{kV}$ and $5 \mathrm{kV}$, respectively. FAIMS separation was carried out by two parallel plates distanced $1.88 \mathrm{~mm}$ apart, with a total path length of $4 \mathrm{~cm}$. A bisinusoidal asymmetric waveform was created by an RF generator (Heartland Mobility) with a peak dispersion voltage of $4 \mathrm{kV}$. Carrier gas flow was produced and maintained by two digital flowmeters (MKS Instruments, Andover, MA, USA) in a $\mathrm{He} / \mathrm{N}_{2}$ mixture (60/40) at a rate of 1.4-2 l/min. The CV was scanned at a rate of 0.33-1 V/min for both positive and negative ionization mode, from 178 to $158 \mathrm{~V}$ and -210 to $-190 \mathrm{~V}$, respectively. Subsequent to the FAIMS device, an ion funnel technology (Heartland Mobility) allowed to transmit and focus the ions generated for maximized sensitivity. Data was acquired at a mass range of $\mathrm{m} / \mathrm{z} 100-600$ and recorded by Thermo Xcalibur Roadmap (Thermo Scientific, version 2.2).

\subsection{Results and discussion}

\section{Girard's reagent T (GT) derivatization of prostanoids}

The GT reagent, used for the prostanoid derivatization, is frequently utilized for the reaction with aldehydes and ketones ${ }^{302}$. GT forms a hydrazone after an acid-catalyzed reaction with a carbonyl group and subsequent loss of water as described previously ${ }^{303-}$ 305. This reaction occurs in a two-step mechanism in which the GT hydrazine first forms a carbinolamine intermediate followed by a dehydration reaction to form a hydrazone. This two-step reaction of GT with $\mathrm{PGE}_{2}$ is displayed in Figure 3.2a and 3.2b, respectively. 
(a)<smiles>CCCCCC(O)/C=C/C1C(O)CC(=O)[C@H]1C/C=C/CCCC(=O)O</smiles>
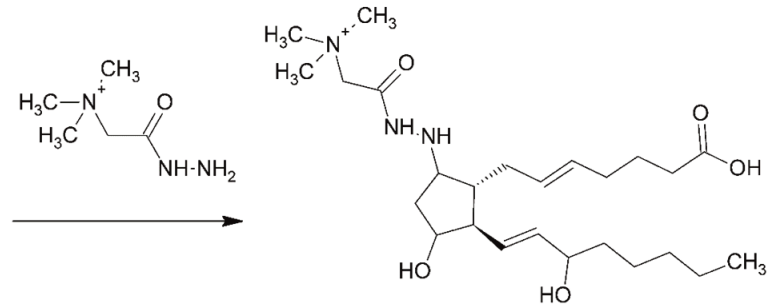

(b)
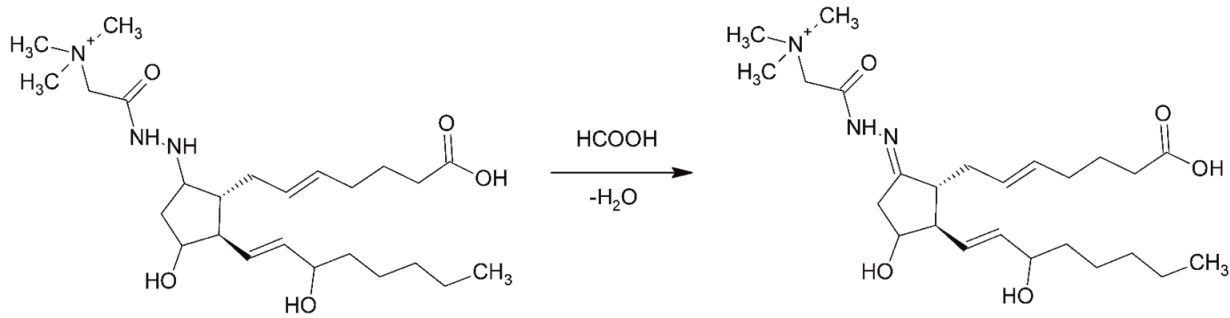

Figure 3.2 The formation of carbinolamine and hydrazine is shown in the two-step mechanism of Girard's Reagent T (GT) derivatization of PGE2. Step 1: the carbonyl group and the GT form a carbinolamine (a). Step 2: under the action of formic acid, a hydrazine is formed after a loss of water (b).

Table 3.1 Prostanoid standard mixture and their exact $m / z$ values.

\begin{tabular}{|c|c|c|}
\hline Compound & {$[\mathrm{M}-\mathrm{H}] \cdot(m / z)$} & {$[M+G T]^{+}(m / z)$} \\
\hline $\mathrm{PGD}_{2}$ & 351.2177 & 466.3184 \\
\hline $\mathrm{PGE}_{2}$ & 351.2177 & 466.3184 \\
\hline 13,14-dihydro-15-keto-PGE2 & 351.2177 & 466.3184 \\
\hline $\mathrm{PGF}_{2 a}$ & 353.2334 & 468.3340 \\
\hline$P D_{1}$ & 353.2334 & 468.3340 \\
\hline $\mathrm{PGE}_{1}$ & 353.2334 & 468.3340 \\
\hline 13,14-dihydro-15-keto-PGF2a & 353.2334 & 468.3340 \\
\hline 6-keto-PGE 1 & 367.2126 & 482.3133 \\
\hline $\mathrm{TXB}_{2}$ & 369.2283 & 484.3289 \\
\hline 6-keto-PGF $1 a$ & 369.2283 & 484.3289 \\
\hline
\end{tabular}

When the carbonyl group in the ring structure of prostanoids reacts with the GT, permanently charged $[\mathrm{M}+\mathrm{GT}]^{+}$ions are formed in the positive ion mode and with a mass difference of $\mathrm{m} / \mathrm{z} 114.1029\left(\mathrm{GT}-\mathrm{H}_{2} \mathrm{O}\right)$ compared to the neutral prostanoid 
molecule. The main advantage of the derivatization with GT is the improved ionization efficiency as the ions are natively charged. Table 3.1 lists the prostanoid standards used in the research, the $\mathrm{m} / \mathrm{z}$ of the $[\mathrm{M}-\mathrm{H}]^{-}$ions (assigned by $\mathbf{m}$ ), and the $\mathrm{m} / \mathrm{z}$ of the $[\mathrm{M}+\mathrm{GT}]^{+}$ ions (assigned by *), with each isomeric prostanoid class assigned by color: red for $\mathrm{m} / \mathrm{z}$ $351 / 466(\square / *)$, green for $m / z 353 / 468(\square / *)$, blue for $m / z 367 / 482(\square / *)$, and purple for $\mathrm{m} / 2369 / 484(-*)$. This will be consistently used throughout the results shown. The mass spectra acquired after direct infusion of derivatized and nonderivatized prostanoid standard mixtures are shown in Figure 3.3. This figure shows the improved ionization efficiency from the GT derivatization when infusing equal concentration of prostanoids. The signal intensity is improved by a factor of $>10$. The $\mathrm{m} / \mathrm{z}$ ratios observed in the mass spectrum (Figure 3.3) show the presence of multiple derivatized prostanoids. The $\left[\mathrm{PGE}_{2}+\mathrm{GT}^{+},\left[\mathrm{PGD}_{2}+\mathrm{GT}\right]^{+}\right.$and $\left[13,14 \text { dihydro-15keto- } \mathrm{PGE}_{2}+\mathrm{GT}\right]^{+}$ions were detected with at $\mathrm{m} / \mathrm{z}$ 466.3; the $\left[\mathrm{PGF}_{2 \mathrm{a}}+\mathrm{GT}\right]^{+},\left[\mathrm{PGE}_{1}+\mathrm{GT}\right]^{+},\left[\mathrm{PGD}_{1}+\mathrm{GT}\right]^{+}$and $[13,14$ dihydro-15keto$\left.\mathrm{PGF}_{2 \alpha}+\mathrm{GT}\right]^{+}$ions were present at $\mathrm{m} / \mathrm{z}$ 468.3. At $\mathrm{m} / \mathrm{z} 482.3$ and $\mathrm{m} / \mathrm{z}$ 484.3, [6keto$\left.\mathrm{PGE}_{1}+\mathrm{GT}\right]^{+}$ions, and $\left[\mathrm{TXB}_{2}+\mathrm{GT}\right]^{+}$and [6keto-PGF $\left.\mathrm{P}_{2 \alpha}+\mathrm{GT}\right]^{+}$ions are shown, respectively. Unbound [GT] $]^{+}$ions were observed at $\mathrm{m} / 2132$.1. 


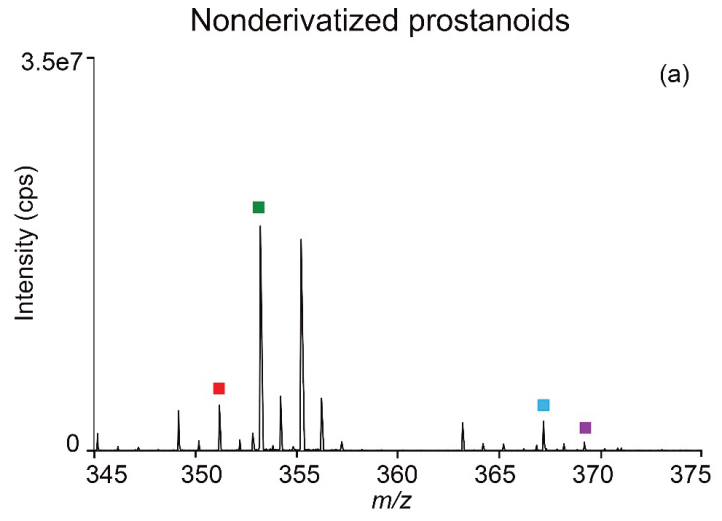

Derivatized prostanoids

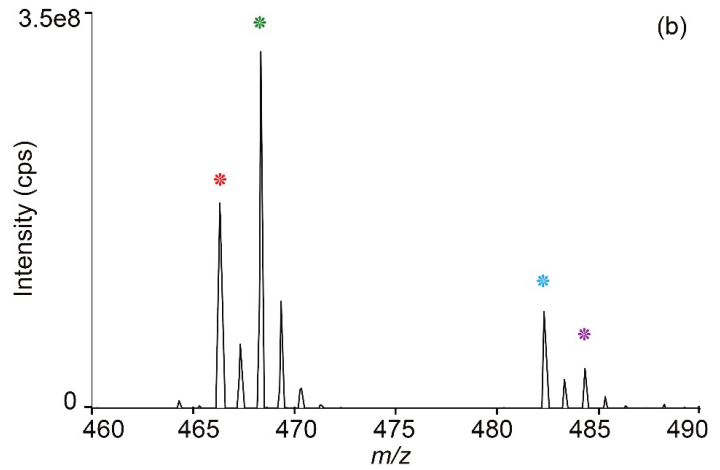

Figure 3.3 Mass spectra of nonderivatized (a) and GT derivatized (b) prostanoid standard mixture. 

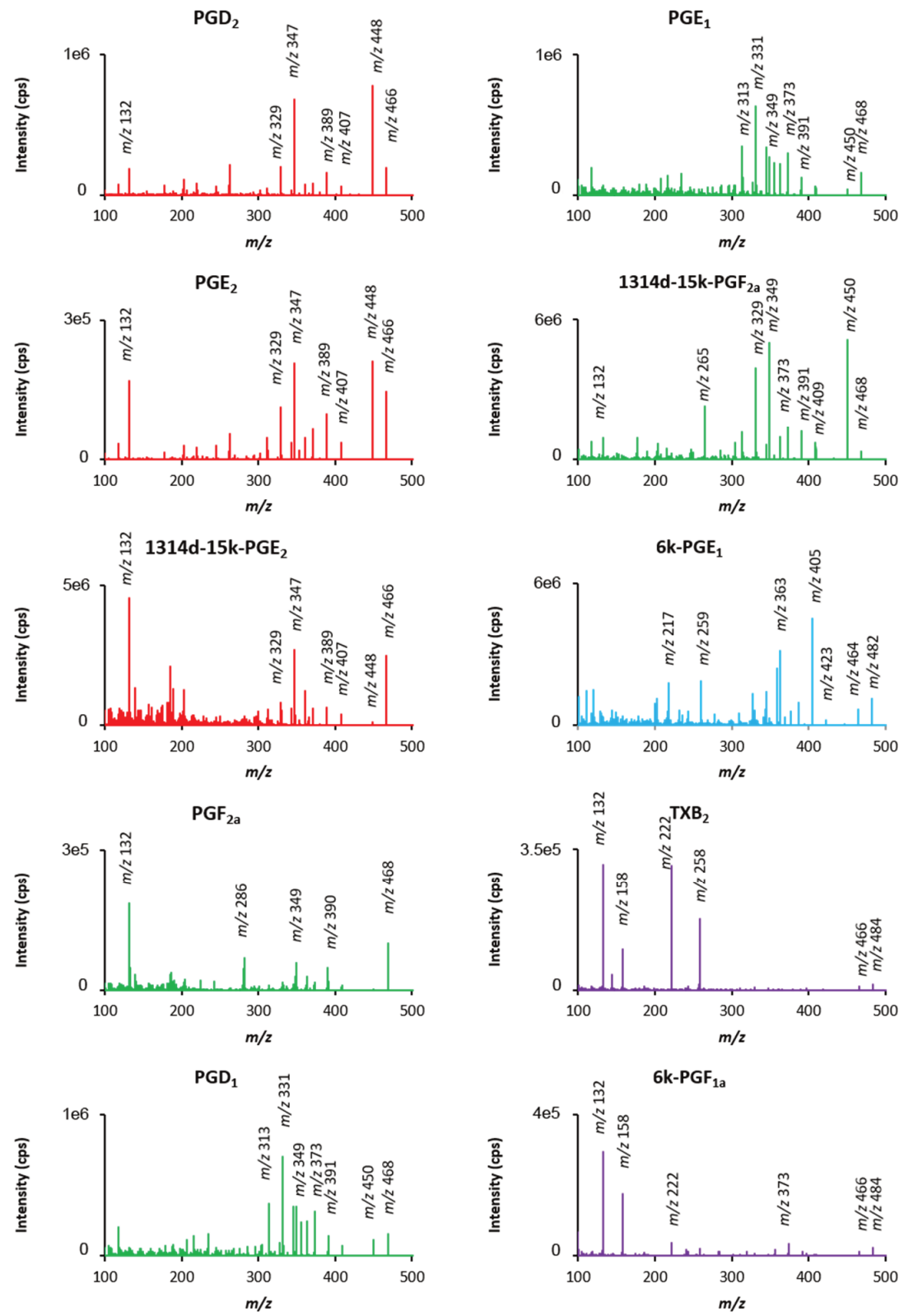

Figure 3.4 High mass resolution MS/MS spectra acquired from individually infused GT derivatized prostanoid standards. The mass spectra are colour assigned consistent with other data shown in this research: $\mathrm{PGD}_{2}$,

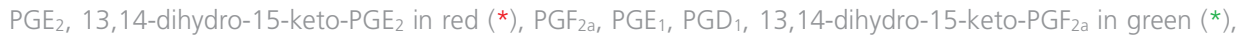
6-keto-PGE in blue $\left({ }^{*}\right)$, and $\mathrm{TXB}_{2}$ and 6-keto-PGF 1 in purple ${ }^{*}$ ). 
Furthermore, our high resolution MS/MS data confirmed the presence of GT derivatized $\mathrm{TXB}_{2}$ and $\mathrm{PGF}_{2 \mathrm{a}}$ in standard mixture (Figure 3.4). This raises the question that the GT derivatization also occurs when compounds do not contain a carbonyl functional group, such as $\mathrm{TXB}_{2}$ and $\mathrm{PGF}_{2 \mathrm{a}}$. Although not described in literature yet, a possible explanation could be that $\mathrm{TXB}_{2}$ and $\mathrm{PGF}_{2 \mathrm{a}}$ are occurring as a keto-enol tautomer, which has been described for the human serum albumin-catalyzed breakdown of 15-keto-PGE 2 at pH 4.4 to the keto-enol tautomer ${ }^{306,307}$. A similar principle could be the case for our acidcatalyzed GT derivatization of $\mathrm{TXB}_{2}$ and $\mathrm{PGF}_{2 \mathrm{a}}$. Even though this keto-enol tautomer seems to be less prone to GT derivatization than a carbonyl group, the possibility cannot be excluded. Brombacher et al. ${ }^{308}$ showed the derivatization of another hydrazine-like reagent with steroids and discussed the preference of the hydrazine nitrogen to perform its nucleophilic attack at the enol form of the carbonyl group rather than at the carbonyl group itself. The fragmentation scheme of Girard's derivatized species has been studied in the past and report the formation of a 6-membered dihydropyridazinone ring and a 5-membered dihydropyrazole ring by a neutral loss of 59 and 87, respectively ${ }^{309,} 310$. Structural identification of GT derivatized TXB 2 is confirmed by the presence of the [M$59]^{+}$and $[\mathrm{M}-87]^{+}$fragment ions in our MS/MS spectra and is shown in Figure 3.5 and 3.6. 

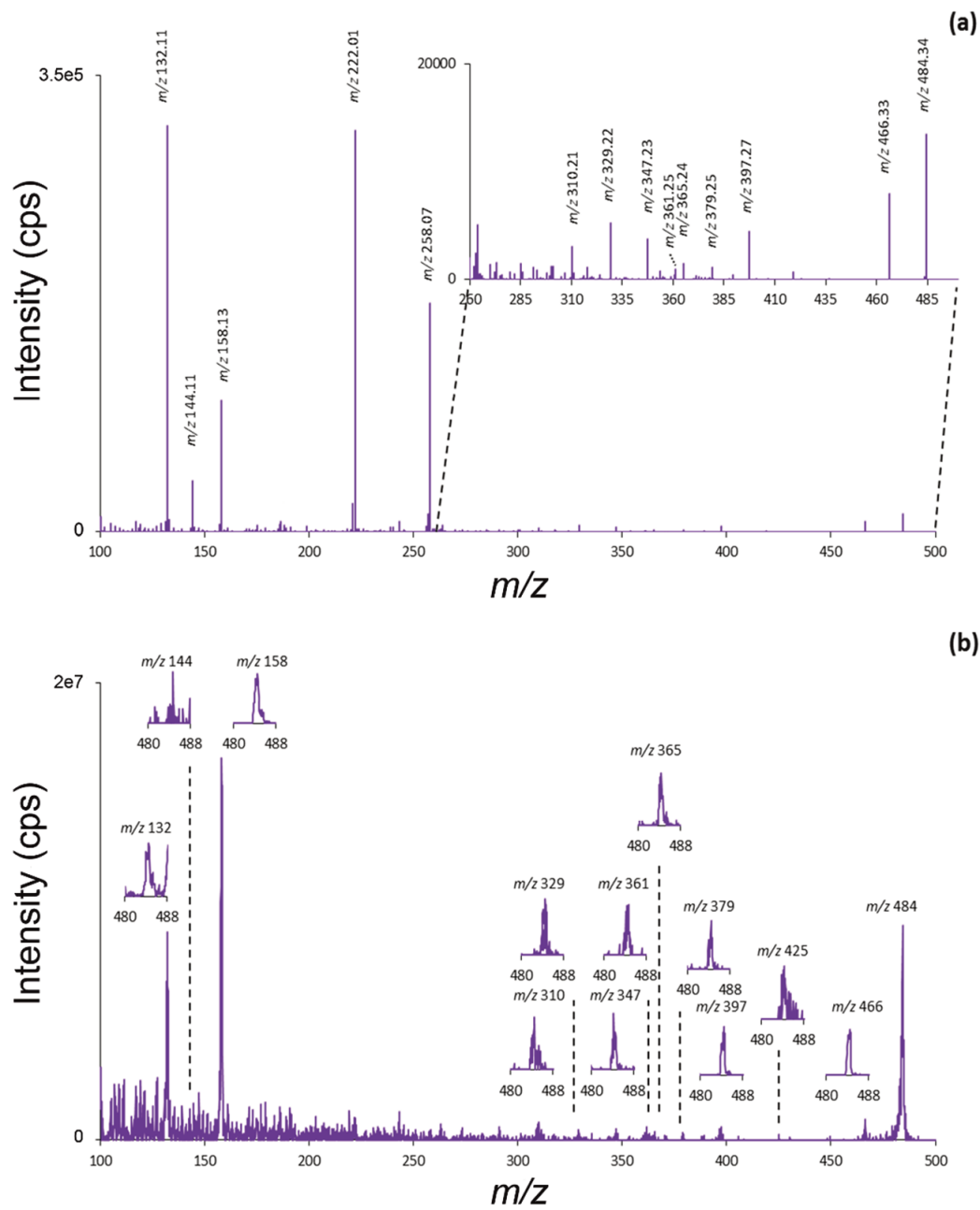

(b)

Figure 3.5 High mass resolution MS/MS (a) and product ion scan (b; labelled with precursor ion scans of each fragment ion) of GT derivatized $\mathrm{TX}_{2}$. The presence of the following fragment ions were observed in both MS/MS spectra: $m / z 132.11, m / z 144.11, m / z 158.13, m / z 310.21, m / z 329.22, m / z 347.23, m / z 361.25, m / z$ 365.24, $\mathrm{m} / \mathrm{z}$ 379.25, $\mathrm{m} / \mathrm{z} 397.27$, and $\mathrm{m} / \mathrm{z}$ 466.33. The $\mathrm{m} / \mathrm{z} 222.01$ and $\mathrm{m} / \mathrm{z} 258.07$ are not considered fragment ions due to their poor mass accuracy (a) and their absence in the product ion scan (b). The $m / z$ 425.30 is not detected in (a) but is present in (b). Structural identification of GT derivatized TXB 2 is confirmed based on the presence of eleven fragment ions and the precursor ion (b). 
(a)<smiles>CCCCCC(=N)CCC1OC(O)CC(O)C1CC=CCCCC(=O)O</smiles>

$[\mathrm{M}+\mathrm{GT}]+$ ion at $\mathrm{m} / \mathrm{z} 484.34$<smiles>CN(C)CC(=O)N=C(CCCCCO)CCC1OC=CC(O)C1CC=CCCCC(=O)O</smiles><smiles>CCCCCC1[NH2+]NC(=O)CC1CC1OC(O)CC(O)C1C/C=C/CCCC(=O)O</smiles>

[M+GT-59]+ ion at $m / 2425.30$ , $\mathrm{CH}_{3} \mathrm{COOH}$<smiles>C=C/C=C/CC1C(O)CC(O)OC1CC1CC(=O)NNC1CCCCCC</smiles>

$[M+G T-119]^{+}$ion at $m / z 365.24$<smiles>C=C/C=C/CC1C(CC2CC(=O)NNC2CCCCC)OC=C[C@@H]1O</smiles>

$[\mathrm{M}+\mathrm{GT}-137]^{*}$ ion at $\mathrm{m} / \mathrm{z} 347.23$<smiles>C=C/C=C/CC1=CC=COC1CC1CC(=O)NN[C@@H]1CCCCCC</smiles>

$[\mathrm{M}+\mathrm{GT}-155]^{+}$ion at $\mathrm{m} / \mathrm{z} 329.22$<smiles>CCCCCC1NN2CC(CC3OC(O)C[C@H](O)C3C/C=C/CCCC(=O)O)C1N2</smiles>

$[\mathrm{M}+\mathrm{GT}-87]^{+}$ion at $m / 2397.27$ $\mid-\mathrm{H}_{2} \mathrm{O}$<smiles>CCCCCC1CC(CC2CNNC2CC=CCCCC(=O)O)OC=C[C@@H]1O</smiles>

$[\mathrm{M}+\mathrm{GT}-105]^{+}$ion at $\mathrm{m} / \mathrm{z} 379.25$<smiles>CCCCCC1NNCC1CC1CNNC1C/C=C/CCCC(=O)O</smiles>

$[\mathrm{M}+\mathrm{GT}-123]^{+}$ion at $\mathrm{m} / \mathrm{z} 361.25$<smiles>CN(C)CC(=O)NN</smiles>

$[\mathrm{GT}]^{+}$ion at $\mathrm{m} / \mathrm{z} 132.11$<smiles>CNC(=O)C[N+](C)(C)C</smiles>

$[\mathrm{GT}+12]^{+}$ion at $\mathrm{m} / \mathrm{z} 144.11$<smiles>CC=NC(=O)CN(C)C</smiles>

[GT+26] $]^{+}$ion at $\mathrm{m} / \mathrm{z} 158.13$ (b)<smiles>CCC/C=N\NC(=O)CC(C)(C)C</smiles>

$[\mathrm{GT}+54]^{+}$ion at $\mathrm{m} / \mathrm{z} 186.16$

Figure 3.6 Structural identification of GT derivatized $\mathrm{TXB}_{2}$ based on high mass resolution MS/MS. The presence of the following fragment ions were observed in the MS/MS spectra (Figure 3.5): $\mathrm{m} / \mathrm{z} 132.11, \mathrm{~m} / \mathrm{z}$ 144.11, $\mathrm{m} / \mathrm{z} 158.13, \mathrm{~m} / \mathrm{z} 310.21, \mathrm{~m} / \mathrm{z} 329.22, \mathrm{~m} / \mathrm{z} 347.23, \mathrm{~m} / \mathrm{z} 361.25, \mathrm{~m} / \mathrm{z} 365.24, \mathrm{~m} / \mathrm{z} 379.25, \mathrm{~m} / \mathrm{z} 397.27$, and $m / z$ 466.33. The lower abundant fragment ions are depicted above (a) and the more stable fragment ions are depicted below (b). Structural identification of GT derivatized $T X_{2}$ is confirmed based on the presence of eleven fragment ions and the precursor ion. 
In summary, the direct infusion experiments confirmed the increased ionization efficiency when comparing derivatized prostanoids with nonderivatized prostanoids. This improvement in ionization is essential for further isomeric prostanoid analysis in the IMS comparison and detection in tissue extracts for sensitivity reasons.

\section{IMS comparison of GT derivatized prostanoid standards}

After improving the ionization efficiency of prostanoids standards by mean of derivatization, we compared different IMS techniques to assess the effect of this derivatization on the ion mobility separation of GT derivatized isomeric prostanoids. Figure 3.7 depicts the IMS spectra assigned by color $(\square / m / m / m)$ for $m / z 466, m / z 468$, $\mathrm{m} / \mathrm{z} 482$ and $\mathrm{m} / \mathrm{z} 484$ values, respectively. A note should be made regarding the different amount of passes in the cIMS experiments. Due to a so-called 'wrap-around' effect of ions, where the fastest ions catch up with the slowest ions in the cIMS loop, a limit number of passes could be performed for the different $\mathrm{m} / \mathrm{z}$ values ${ }^{311}$. For the identification of the derivatized prostanoids standards, MS/MS spectra were acquired using high resolution MS (Figure 3.4) and compared with the fragment ions found in the IMS-MS analyses. Only identified peaks were labeled in the IMS spectra, based on infusion of individual standards followed by MS/MS fragmentation.
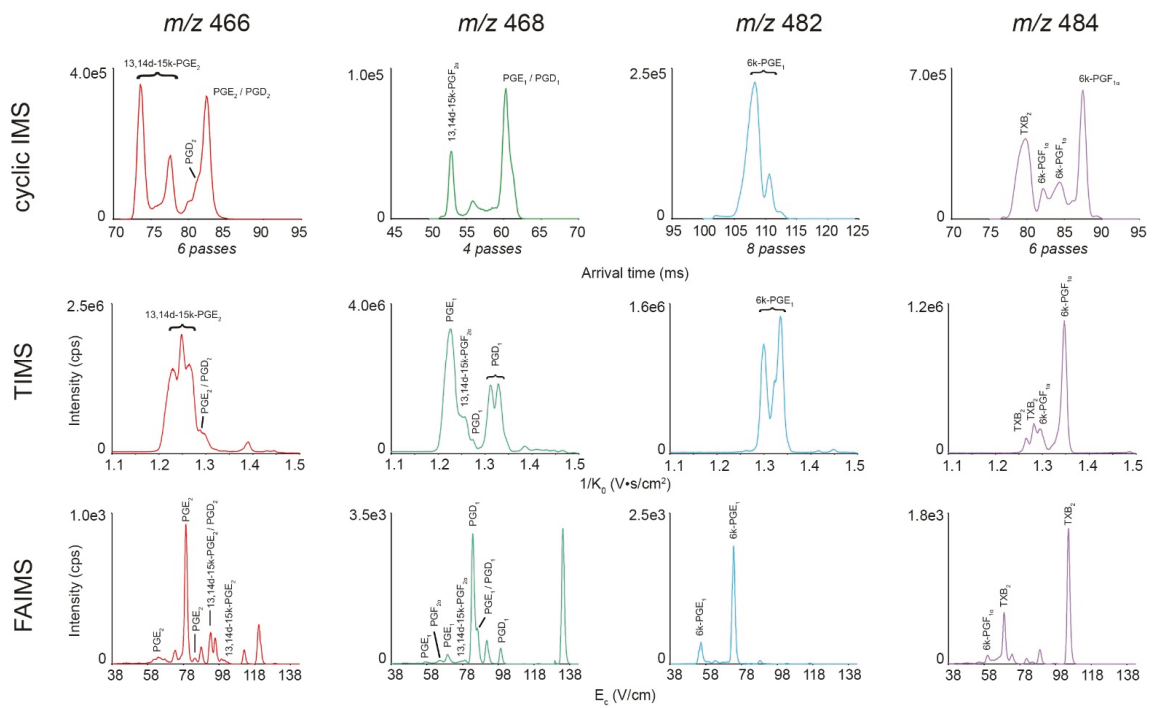

Figure 3.7 High resolution IMS spectra of a GT derivatized prostanoid standard mixture: cIMS, TIMS and FAIMS analyses show peaks at $m / 2466, m / z 468, m / z 482$ and $m / z 484$ for multiple isomers which are labelled in the spectra. Identification is confirmed by MS/MS and infusion of individual standards. 
Significant differences are observed between the three IMS, such as the number of detected isomers. For instance for $m / z 466,4,5$ and 10 peaks are observed in the cIMS, TIMS and FAIMS spectra, respectively. FAIMS spectra consistently show more and better separated peaks throughout the spectra. This could be explained by the fact that the separation in FAIMS is based on nonlinear IMS while TIMS and cIMS are based on linear IMS. The latter two techniques operate at low electric fields leading to a linear relationship between the ion's velocity and the electric field applied. FAIMS rapidly switches between a low and high electric field in an asymmetric manner which results in a nonlinear relationship between the velocity and the electric field. Nonlinear IMS is much more orthogonal to mass spectrometry than linear IMS hence the improved separation ${ }^{72}$. Therefore, our FAIMS spectra show a much larger amount of peaks after infusion of the same isomeric standard mixture. Another significant difference between our FAIMS and TIMS/cIMS spectra is the difference in intensity due to environmental conditions. Both CIMS and TIMS are performed under vacuum conditions while FAIMS operates at atmospheric pressure. Although FAIMS provides the largest resolving power (partly due to atmospheric conditions), this atmospheric environment leads to a significant loss in ion transmission. When comparing TIMS and CIMS, minor variations in isomeric separation could be observed due to the 'reversed' mechanism of both IMS techniques. In cIMS, the electric field transports ions through the buffer gas while TIMS uses the electric field to trap ions as a carrier gas transports them through the IMS cell. This 'reversed' principle between cIMS and TIMS explaines that the ion's path length is generated differently by optimizing gas velocity and time. A longer path in cIMS is established by more passes and, as a result, a physically longer path (50-120 ms arrival time). In TIMS, a longer path is accomplished by a longer trapping time (450 ms trapping time). The IMS separation time scale should be considered when comparing CIMS and TIMS. In FAIMS, this is in the range of minutes (>20 min).

For identification of prostanoids, some of the isomeric prostanoids can be separated based on specific MS/MS fragment ions alone, such as $P G E_{1} / P G D_{1}$ from $P G F_{2 a}$. However, this is not possible for the separation of $\mathrm{PGE}_{1}$ from $\mathrm{PGD}_{1}$ nor of $\mathrm{PGE}_{2}$ from $\mathrm{PGD}_{2}$ using MS/MS alone. From a biological point of view, this isomeric separation is crucial in the study of the different metabolic pathways of prostanoids (e.g. COX-1 and COX-2)258. FAIMS is the most successful in the separation of these crucial isomeric prostanoids. In addition, multiple peaks for one compound are observed in almost all IMS spectra. This could be a result of the ability of IMS to not only separate isomers but also stereoisomers, in particular for FAIMS separation. This could be due to the drastic changes in the $K_{\text {high }} / K_{\text {low }}$ ratio. Literature shows that FAIMS is able to separate the cis/trans stereoisomers ${ }^{289,} 296$ as well as diastereomers ${ }^{312}$. 
$m / z 468$

$m / z 482$

$m / 2484$
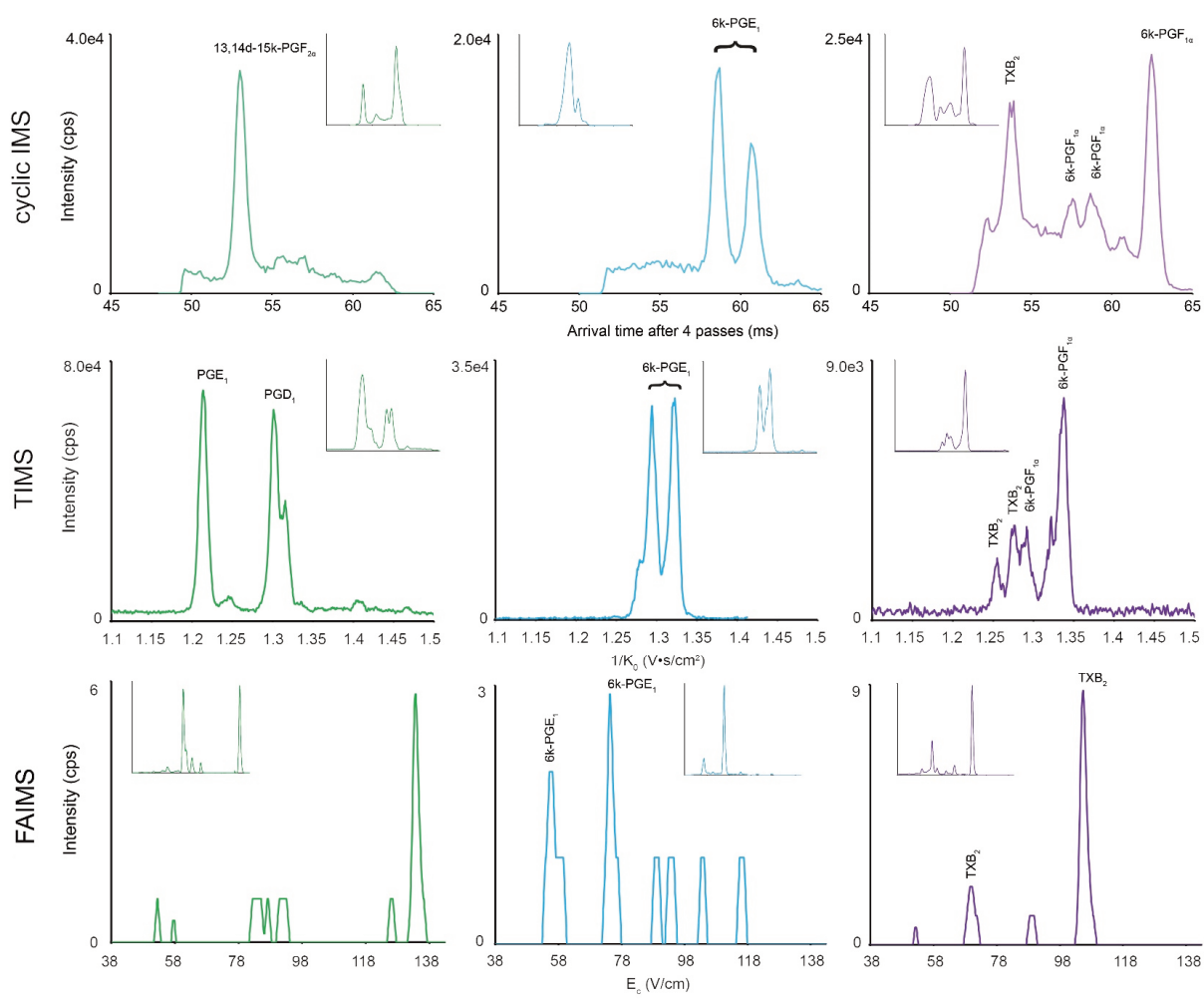

Figure 3.8 High resolution IMS spectra of a GT derivatized mini pig intestinal tissue extract: cIMS, TIMS and FAIMS analyses show peaks at $\mathrm{m} / \mathrm{z} 468, \mathrm{~m} / \mathrm{z} 482$ and $\mathrm{m} / \mathrm{z} 484$ for multiple isomers which are labelled in the spectra. Identification is confirmed by MS/MS and by comparison with prostanoid standards.

\section{Prostanoids in mini pig intestine tissue extracts}

Endogenous prostanoids were investigated here in a mini pig intestine tissue extract using CIMS, TIMS and FAIMS to assess their detectability in biological matrices using the previously optimized method. Figure 3.8 shows the IMS spectra assigned by color ( $\square / \square / \square)$ of $m / z 468, m / z 482$ and $m / z 484$ after direct infusion of GT derivatized mini pig intestine tissue extracts. Peaks were identified based on direct infusion of individual standards and MS/MS spectra. The aspects mentioned in the IMS comparison apply here more extensively. Even with the GT derivatization, one could argue that the occurrence of ion suppression from the sample matrix is still a point of attention. The combination of lower total ion transmission due to atmospheric conditions and the suppression from matrix ions causes a limited number of detected analyte ions after FAIMS analysis. However, the modification of the ion chemistry by the GT derivatization changes the 
ionization competition occurring in the gas phase and, therefore, promotes the ionization of the derivatized prostanoids. This is shown in the FAIMS comparison of GT derivatized and nonderivatized compounds (Figure 3.9). Despite the possibility of ion suppression from tissue matrix and a loss in total ion transmission from FAIMS to MS, low-level prostanoids (5-100 ng/g tissue determined by quantitative LC-MS) were detected in tissue extracts. We see potential in FAIMS-MS as a powerful tool in combination with chromatography for isomeric separation of prostanoids.

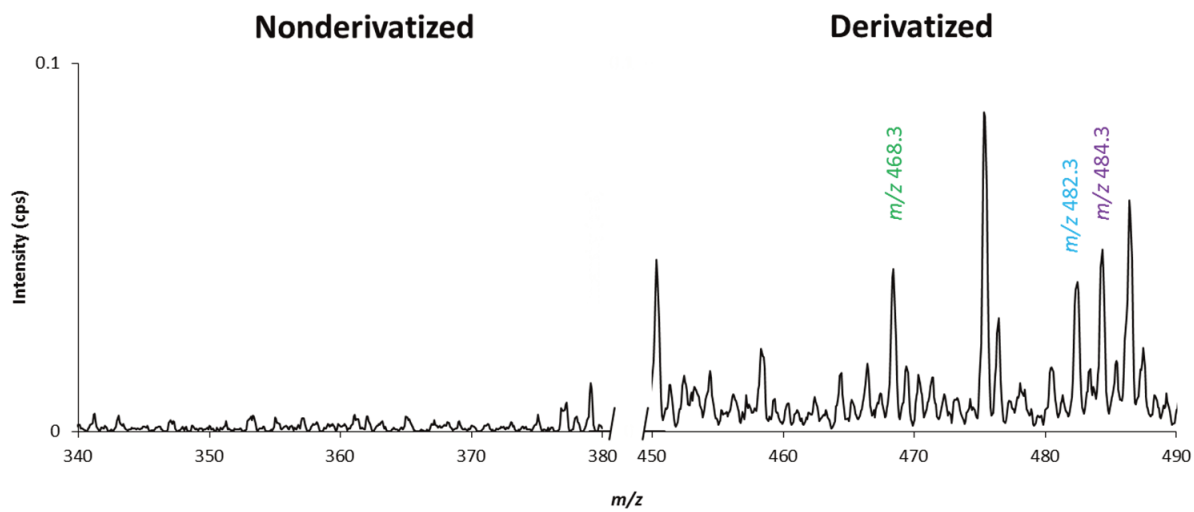

Figure 3.9 Comparison of derivatized and nonderivatized prostanoids in tissue extract. The mass spectrum of the GT derivatized tissue extract shows peaks at $m / z 468.3(*), m / z 482.3(*)$, and $m / z 484.3(*)$. No prostanoid peaks are observed in the mass spectrum of the nonderivatized tissue extract.

In linear IMS, the GT derivatization provides an opportunity for endogenous detection of prostanoids. $6 \mathrm{k}-\mathrm{PGE} \mathrm{E}_{1}$ was detected at $\mathrm{m} / z 482$ and $\mathrm{TX}_{2}$ and $6 \mathrm{k}-\mathrm{PGF}_{2 \mathrm{a}}$ were observed at $m / z$ 484. At $m / z$ 468, different prostanoids are detected in the tissue extract after CIMS and TIMS. This is interesting considering that the ionization technique is the same, the IMS separation is identical to that applied to the prostanoid standards and the mass resolving power is similar. Interfering background ions from the sample matrix could mask the presence of prostanoids. IMS is known to decrease the level of contaminating background ions and could, due to the different mechanisms of both techniques, remove different background ions and, therefore, reveal the presence of the prostanoids.

\subsection{Conclusion}

This work demonstrates that high resolution IMS is a very powerful approach for the separation and identification of prostanoids present in complex matrices like biological tissues. Chemical derivatization with GT reagent significantly contributed to improve 
the ionization efficiency of both standards and endogenous prostanoids, and thus improve sensitivity for their detection in tissue samples. The increase in signal intensity in the mass spectra by GT derivatization demonstrates the significant improvement in ionization despite the possible occurrence of ion suppression. The comparison between CIMS, TIMS and FAIMS revealed the strengths and weaknesses of all three IMS techniques in which a clear distinction is observed between linear and nonlinear IMS. Linear IMS provides the best sensitivity for endogenous analysis while nonlinear IMS delivers the highest IMS resolution. The combination of significantly improved ionization efficiency and isomeric separation power by high resolution IMS after GT derivatization confirms its applicability for low-level prostanoid analysis without the need of expensive and time-consuming sample clean up. Our derivatization strategy offers high sensitivity and excellent stereoisomeric separation for screening of complex biological systems. High resolution IMS, in combination with other orthogonal analytical techniques, could further unravel the role of isomeric prostanoids in biology and disease. 


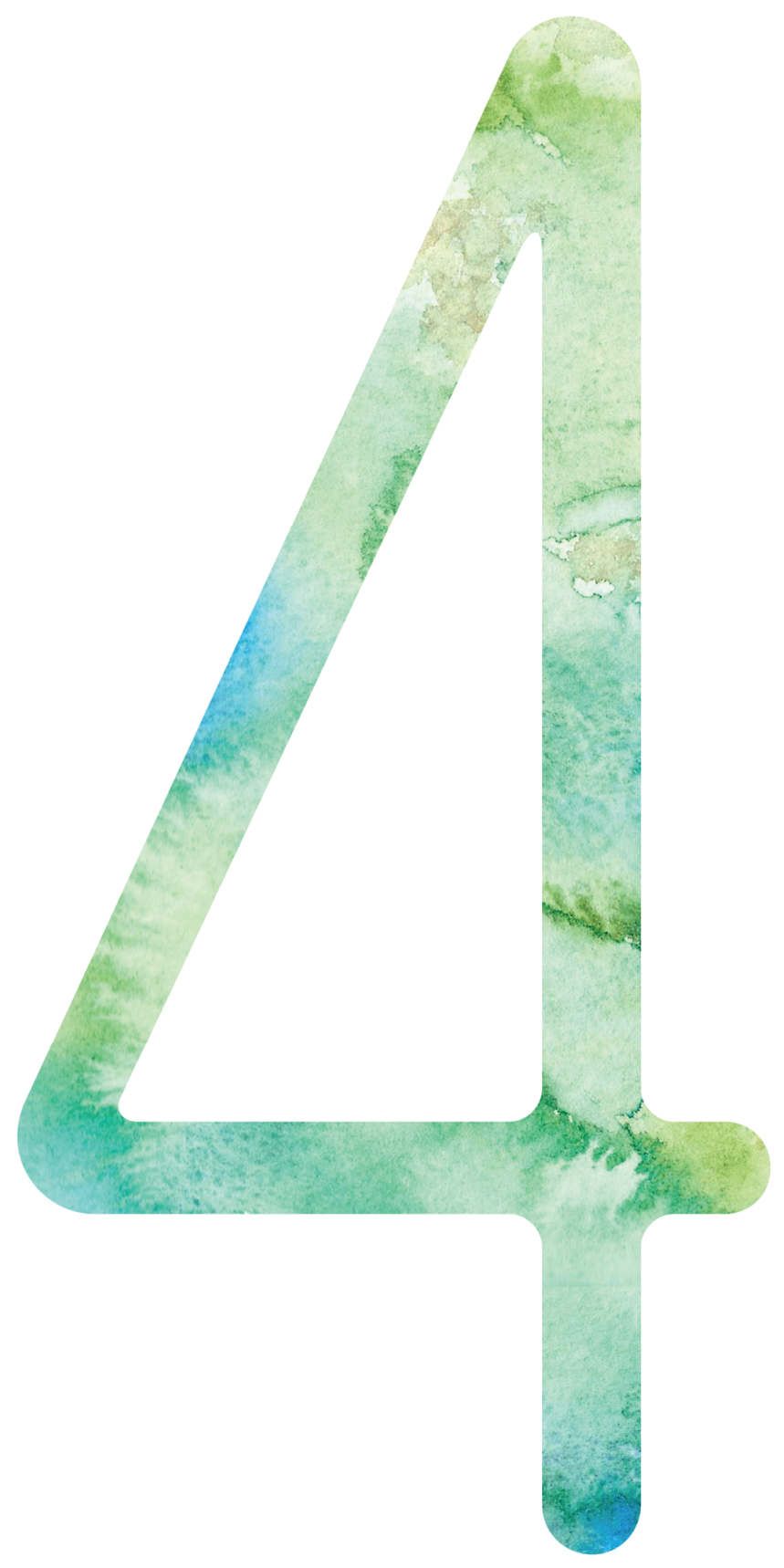




\section{Targeted Drug and Metabolite Imaging}

Based on

Targeted Drug and metabolite imaging: Desorption Electrospray Ionization Combined with Triple Quadrupole Mass Spectrometry

Lieke Lamont, Gert B. Eijkel, Emrys A. Jones, Bryn Flinders, Shane R. Ellis, Tiffany Porta Siegel, Ron M.A. Heeren and Rob J. Vreeken

Analytical Chemistry 201890 (22), 13235-1323. 
Mass spectrometry imaging (MSI) has proven to be a valuable tool for drug and metabolite imaging in pharmaceutical toxicology studies and can reveal for example accumulation of drug candidates in early drug development. However, the lack of sample cleanup and chromatographic separation can hamper the analysis due to isobaric interferences. Multiple reaction monitoring (MRM) uses unique precursor ionfragment ion transitions to add specificity which leads to higher selectivity. Here, we present a targeted imaging platform where desorption electrospray ionization (DESI) is combined with a triple quadrupole (QqQ) system to perform MRM imaging. The platform is applied to visualize i) lipids in mouse brain tissue sections and ii) a drug candidate (B) and metabolite (A) in canine liver tissue. All QqQ modes are investigated to show the increased detection time provided by MRM as well as the possibility to perform dual polarity imaging. This is very beneficial for lipid imaging since some phospholipid classes ionize in opposite polarity (e.g. PC/SM in positive mode and PS/PE in negative mode). Drug and metabolite images are obtained to show its strength in drug distribution studies. Multiple MRM transitions are used to confirm the local presence and selective detection of pharmaceutical compounds. 


\subsection{Introduction}

Drug discovery and development aims to find suitable candidates that can inhibit or activate a pathway to have a positive effect in a disease state ${ }^{82}$. The desired effect is the potential drug being distributed to the target site and bound to the biological receptors present for a certain amount of time at a high enough concentration ${ }^{83}$. However, if a drug candidate, or its metabolite(s), accumulates in tissue it can result in significant toxicological effects. In addition, these effects can also derive from the presence of specific (secondary) drug metabolites. Therefore, toxicology studies are key in deciding whether a potential drug will be further developed ${ }^{141}$. Analytical tools are thus required for species identification and localization. Current imaging technologies, like quantitative whole-body autoradiography (Q-WBA), examine drug distribution but require radiolabeling and therefore cannot distinguish between possible drugs and its metabolites $^{142}$.

Mass spectrometry imaging (MSI) allows label-free drug distribution investigation because of its ability to map drug candidates and metabolites simultaneously ${ }^{80,83,141 .}$ Matrix-assisted laser desorption ionization (MALDI) is often combined with MSI due to its high spatial resolution, sensitivity and throughput. However, difficulties in MALDIMSI can arise due to inhomogeneity in matrix application and the possible interferences arising from matrix-related signals, especially in the absence of high mass resolving power. Lately, ambient liquid ionization techniques have complemented MALDI-MSI, as they do not require external matrices to facilitate analysis. Desorption electrospray ionization (DESI) ${ }^{117}$ was introduced by Cooks and co-workers and has established itself as the main ambient tool for MSI. DESI uses an electrospray emitter to map extracted molecules directly from the cryo-sectioned tissue sample. The main advantages of DESI are the minimal sample preparation required prior to analysis and its compatibility with histological staining ${ }^{131}$. DESI imaging of lipids $^{99,} 115$ is the most popular but the pharmaceutical industry has gained interest in the technology ${ }^{313}$ for applications like drug blood-brain barrier permeability ${ }^{314}$ and cassette dosing ${ }^{155}$.

Independent of the ionization source, constant technical improvements are made to improve MS detection. The integration of ion mobility spectrometry (IMS) $)^{228,315}$ with MSI increases selectivity by reducing the interference from isobaric background compounds. Not only IMS but also using high mass resolution MSI ${ }^{316,317}$ allows the separation of isobaric interference from targeted analytes, which is beneficial for drug analyses where chemical background from biological samples is a real burden. Even though the mass resolving capability of the newest high resolution instruments is significant, examples are found in literature of isobaric compounds that could not be separated $^{130}$. In consequence, a more selective tandem MS (MS/MS) approach is 
required to separate these isobaric compounds and are generally executed using a QqQ instrument. Although referred to as a triple quadrupole instrument $(\mathrm{QqQ})$ by mass spectrometrists, most commercially available QqQ instruments nowadays only use two quadrupole mass analyzers and, therefore, are often called tandem quadrupole (TQ) instruments ${ }^{3}$. In consistency with the MS field, we refer to the system as a QqQ instrument ${ }^{318}$. The QqQ uses the first quadrupole (Q1), followed by a collision cell (q) being activated or de-activated, and second quadrupole (Q3) to either select or scan ions. The system can be used in different modes ${ }^{25,319}$ : MS scan (Q1 operates in RF only mode, no fragmentation, Q3 is scanning), product ion scan (Q1 is selecting, Q3 is scanning; PROD), precursor ion scan (Q1 is scanning, Q3 is selecting; PREC), neutral loss scan (Q1 is scanning and Q3 is scanning with an offset specific for a neutral fragment; $\mathrm{NL})$, and multiple reaction monitoring (Q1 and Q3 are selecting; MRM). Once the precursor and fragment ions are known, a targeted and high-throughput MRM method can analyze multiple compounds simultaneously. In addition, the system enables dual polarity imaging in one experiment. Polarity switching has been explored previously for lipid MS imaging ${ }^{223}$. MALDI-QqQ imaging has also improved sensitivity for drug distributions studies ${ }^{173,320}$. Other MALDI-MRM drug applications have been focused on skin analysis ${ }^{321}$ and forensics ${ }^{322,}$ 323. MRM imaging allows for quantitative MSI approaches due to its wide dynamic range ${ }^{203}$.

Here we report the benefits of using a QqQ mass spectrometer for targeted DESI-MS imaging, which is illustrated with different sets of experiments. First, the improved specificity of MRM is compared with the QqQ scanning modes for multiple lipid classes in rat brain tissue. In addition, DESI-MRM is applied to map two drug candidates (A and B) in canine liver. At last, lipid imaging of rat brain tissue is performed to prove the benefit of dual polarity imaging.

\subsection{Experimental section}

\section{Materials and reagents}

ULC/MS-grade water $\left(\mathrm{H}_{2} \mathrm{O}\right)$, ULC/MS-grade methanol $(\mathrm{MeOH})$, ULC/MS-grade ethanol and $99 \%$ formic acid (FA) were purchased from Biosolve (Valkenswaard, NL). Microscopic glass slides were purchased from Thermo Scientific (Braunschweig, DE). Drug candidate standards (compound A and B) was provided by Janssen R\&D (Beerse, $\mathrm{BE})$.

\section{Tissue collection and preparation}

Rat brain and canine liver were used in this research. MAASTRO clinic (Maastricht, NL) provided Wistar Albino Glaxo Rat brain tissues that were snap frozen in liquid nitrogen. 
Janssen $R \& D$ (Beerse, $B E$ ) provided canine liver tissues dosed with a drug candidate (B) at $65 \mathrm{mg} / \mathrm{kg}$. In vivo, drug B metabolizes into a dealkylated metabolite identical to the structure of compound A. For confidentiality reasons, structural information of the drug candidates cannot be revealed. All tissue samples were stored at $-80^{\circ} \mathrm{C}$ until cryosectioning. Fresh-frozen tissues were cryo-sectioned using a Microtome cryostat (Thermo Scientific, Braunschweig, DE) into 12- $\mu \mathrm{m}$ thick tissue sections and thaw mounted on microscope glass slides, stored at $-80^{\circ} \mathrm{C}$, and air-dried prior to DESI-QqQ analysis.

\section{DESI-QqQ instrumentation}

A Xevo TQ-S micro was equipped with a DESI source for the MSI analysis (Waters). DESI solvent $\left(\mathrm{MeOH} / \mathrm{H}_{2} \mathrm{O}, 98 / 2\right.$, v/v) was supplied by a Waters ACQUITY UPLC M-class binary solvent manager at 2-5 $\mathrm{\mu} / \mathrm{min}$. For positive ionization experiments, $0.1 \% \mathrm{FA}$ was added to the DESI solvent. General parameters were: $N_{2}$ nebulizing gas pressure $=3-5$ bar; spray voltage $= \pm 3-4 \mathrm{kV}$; MS source temperature $=150^{\circ} \mathrm{C}$; sampling cone voltage $=25$ V. A custom-built inlet capillary was heated to $300-500^{\circ} \mathrm{C}$. Pixel sizes differed per MSI analysis from 30-100 $\mu \mathrm{m}$. Mass resolution settings were optimized to unit resolution. NL/PREC/PROD scan times ranged from 0.995-2.496 sec/pixel and MRM dwell times ranged from 0.199-0.999 sec/pixel. All images were acquired with Omni Spray 2-D version 2.0.1 (Prosolia) combined with MassLynx version 4.1 (Waters, Milford, MA, USA).

\section{H\&E staining}

Hematoxylin and eosin (H\&E) staining was executed on the same tissue sections after DESI-QqQ analysis. The tissue sections were washed in successive EtOH baths (100\%, $96 \%, 70 \%$ ) and deionized water for 3 min each. Hematoxylin (Merck, Darmstadt, DE) staining was executed for 3 min followed by a gentle 3 min wash with running tap water. Eosin staining is performed for $30 \mathrm{sec}$, followed by a gentle wash with running tap water for $3 \mathrm{~min}$. The protocol was finalized by a $1 \mathrm{~min} \mathrm{EtOH}$ wash and a $30 \mathrm{sec}$ xylene wash for dehydration. Cover slips were placed on the stained tissues using Entellan. A MIRAX Desk scanner (Zeiss, Gottingen, DE) was used to acquire optical images.

\section{Data processing and visualization}

For all QqQ imaging experiments, individual line scans were acquired. For individual MRM transitions, all raw line scan files were converted into mzML with MSConvertGUI (Vanderbilt University, Nashville, TN, USA) and further processed by an in-house written 
MATLAB script (v. R2015a). To visualize NL/PREC/PROD images, raw line scan files were directly processed by a MATLAB script.

\subsection{Results and discussion}

\section{Scanning versus MRM}

MRM mode was investigated and compared with several scanning modes to target multiple high and low abundant lipid classes $^{324}$. First, a DESI-NL scan of 87, corresponding to deprotonated phosphatidylserine (PS) lipids, was performed ${ }^{325}$. This was followed by a DESI-MRM imaging experiment (consecutive tissue section) of 5 corresponding MRM transitions selected from the NL spectrum (Figure 4.1a). The same strategy was used for the experiments in negative ion mode. In positive ion mode, a DESI-PREC imaging experiment was executed to scan for precursors with the $m / z 184$ fragment ion ${ }^{324}$ corresponding to protonated phosphatidylcholine (PC) and sphingomyelin (SM) lipids. Based on the PREC spectrum (Figure 4.1b) 5 lipid species at variable abundance were selected. The corresponding MRM transitions were used in a DESI-MRM imaging experiment on a consecutive tissue section.

(a)

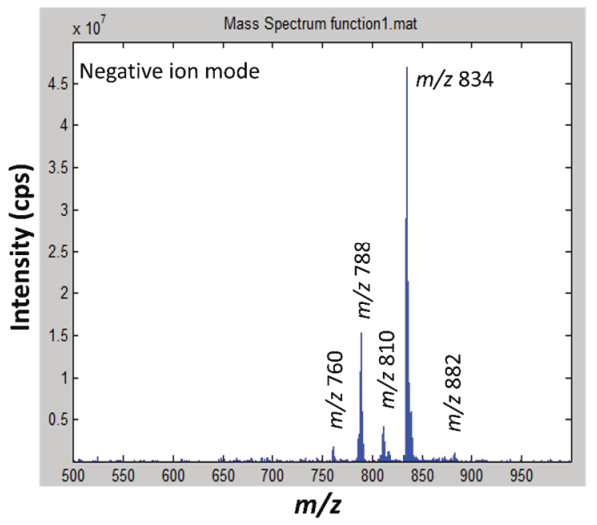

\section{(b) PREC spectrum of $m / z 184$}

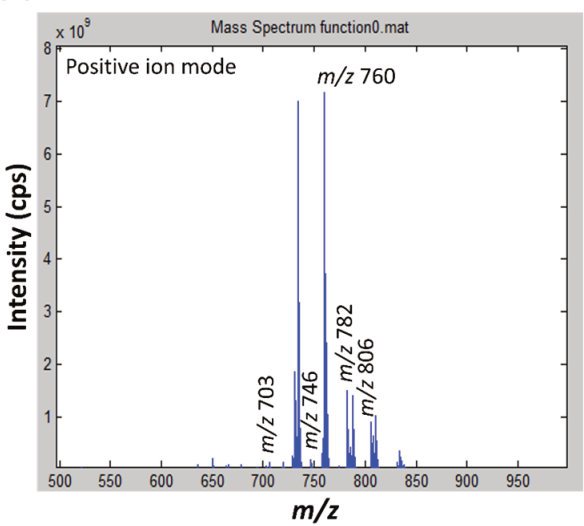

Figure 4.1 NL spectrum (a) of 87 acquired from rat brain in negative ion mode. PREC spectrum (b) of $\mathrm{m} / \mathrm{z}$ 184 acquired from rat brain in positive ion mode. Corresponding images are shown in Figure 4.2.

Figure 4.2 shows the resulting MS/MS images of ten lipid species of which five were obtained in positive ion mode (PC and SM, left 2 columns) and five in negative ion mode (PS, right 2 columns). Using MRM for MS imaging experiments significantly adds specificity to the analysis compared to MS scan. The terms specificity and selectivity are often confusingly used. Selectivity is the ability of a method to distinguish between the 
analyte and its sample matrix. Specificity is the ability to measure one specific analyte in presence of its sample matrix (i.e. near $100 \%$ selectivity for one specific analyte) $)^{326}$. With MRM imaging, the selection of the fragment ion corresponding to the precursor ion adds specificity to the analyte and, therefore, measures this compound at near 100\% selectivity. This allows separation of compounds with higher selectivity than current high mass resolving power MS technologies. Lanekoff et al. have combined nanospray DESI with multiplexed MS/MS imaging ${ }^{126}$

In this work, MRM could separate both isobaric metabolites and isobaric lipids where current high mass resolving power MS failed. An example is the separation of the $[\mathrm{M}+\mathrm{Na}]^{+}$ion of $\mathrm{PE}(\mathrm{P}-16: 0 / 22: 6)$ at $\mathrm{m} / 2770.5095$ from the $[\mathrm{M}+\mathrm{K}]^{+}$ion of $\mathrm{PC} 32: 1$ at $\mathrm{m} / \mathrm{z}$ 770.5097. The use of MRM versus PREC/NL scanning modes increases the total detection time per precursor ion. For instance, in NL scanning mode, [PS(44:10)-H]- is acquired at an acquisition rate of $20,000 \mathrm{Da} / \mathrm{sec}$ over a $\mathrm{m} / \mathrm{z} 500-1000$ mass range at a scan rate of $2.496 \mathrm{sec} /$ pixel. This leads to a total detection time of $62.4 \mathrm{~ms} / \mathrm{pixel}$ for the [PS(44:10)-H] ion. In MRM mode, at the same total analysis time, the image of [PS(44:10)-H] was acquired at $999 \mathrm{~ms} / \mathrm{pixel}$. This results in MRM images from lowabundant lipid species $(<1 \%$ for PS(44:10)) that are poorly detected in NL scanning mode. Not only is DESI-MRM imaging more selective but also significantly reduces analysis costs relative to high mass resolving MS imaging of isobars; which can be an argument for implementation in the pharmaceutical industry. 
Positive ion mode

PREC 184
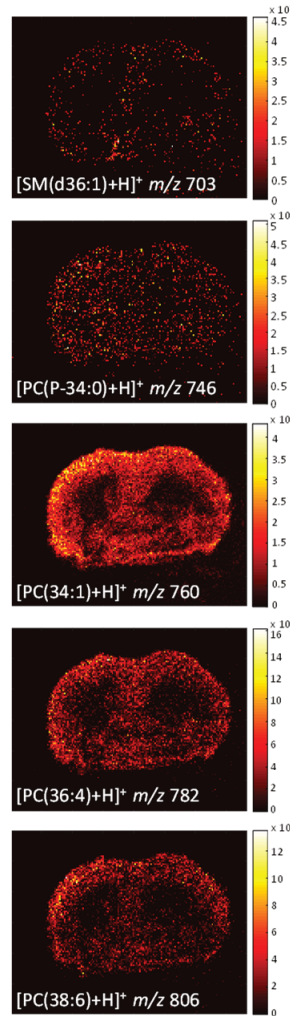

$\underline{2 \mathrm{~mm}}$

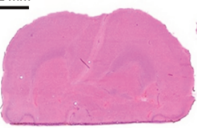

MRM
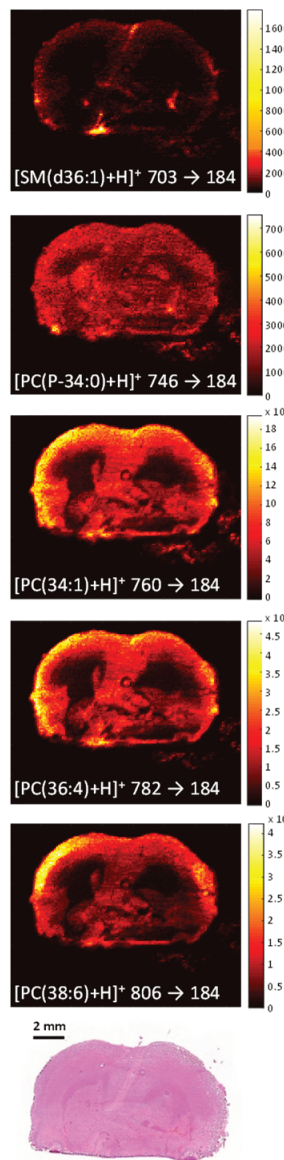

Negative ion mode

NL 87
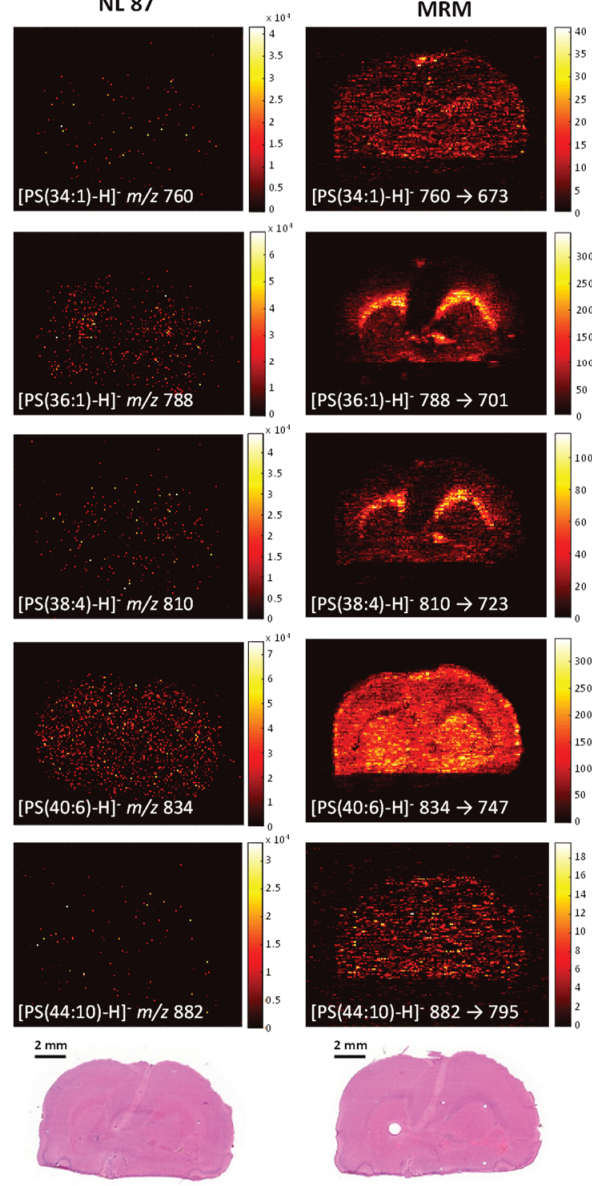

Figure 4.2 TIC images acquired in scanning and MRM modes. Left two columns show images acquired in positive mode, and the right two columns show images acquired in negative mode. In the left two columns, DESI-PREC images (scan time 0.995 s) are compared with DESI-MRM images (dwell time 0.199 s/MRM) of the same species. The right two columns show the DESI-NL images (scan time $2.496 \mathrm{~s}$ ) and their related DESIMRM images (dwell time $0.999 \mathrm{~s} / \mathrm{MRM}$ ). Pixel size is $100 \mu \mathrm{m}$. H\&E images are displayed at the bottom of each column.

\section{Confirmation of spatial localization of drug and metabolites}

Due to the additional specificity of DESI-MRM imaging, this technology is a suitable tool to pharmaceutical studies. The canine liver tissues used for this MRM imaging application are obtained from an investigation of a possible drug candidate for prostate cancer: Compound B with a molecular weight of $515 \mathrm{~g} / \mathrm{mol}\left([\mathrm{B}+\mathrm{H}]^{+}\right.$ion at $\left.\mathrm{m} / \mathrm{z} 516\right)$ which is metabolized into the demethylated metabolite $A$ at $501 \mathrm{~g} / \mathrm{mol}\left([\mathrm{A}+\mathrm{H}]^{+}\right.$ion at 
$m / z 502$ ). Based on preliminary experiments performed in Janssen R\&D, a NL spectrum of 418 was obtained followed by a PROD spectrum of compound A. This information was used in the selection of two specific MRM transitions for each compound $(\mathrm{m} / z 516$ $\rightarrow m / z$ 98, $m / z 516 \rightarrow m / z 488 ; m / z 502 \rightarrow m / z 84, m / z 502 \rightarrow m / z 474$ ). To confirm spatial distribution, all MRM transitions are used to map the drug candidate and its metabolite. Figure 4.3 shows two MRM images of compound A (Figure 4.3a and 4.3b) and two of metabolite A (Figure 4.3c and 4.3d). All MRM images reveal significant accumulation of $A$ and $B$ in tissue lesions, which are visible in the H\&E images and highlighted by arrows in figure 4.3e. To complement the DESI-MRM data, additional MALDI-MSI experiments were executed and display similar distribution of $A$ and $B$ (Figure 4.4).

The accumulation of compound B correlates with histological lesions previously annotated by the pathologist and indicates severe fibrosis which resulted in the exclusion of compound B from further development. It is worth mentioning that the occurrence of ion suppression and/or enhancement due to the presence of bile acids in the bile duct may influence the detected levels of compound $\mathrm{B}^{201}$. However, qualitative MSI provides pharmaceutical researchers with unique understanding on drug distribution ${ }^{142}$. This qualitative information benefits significantly from selective MS detection. Our data shows that MRM imaging can not only be used for this selective detection of drugs and metabolites but also for spatial confirmation (e.g. accumulation at the same location) by monitoring multiple MRM transitions per compound. 

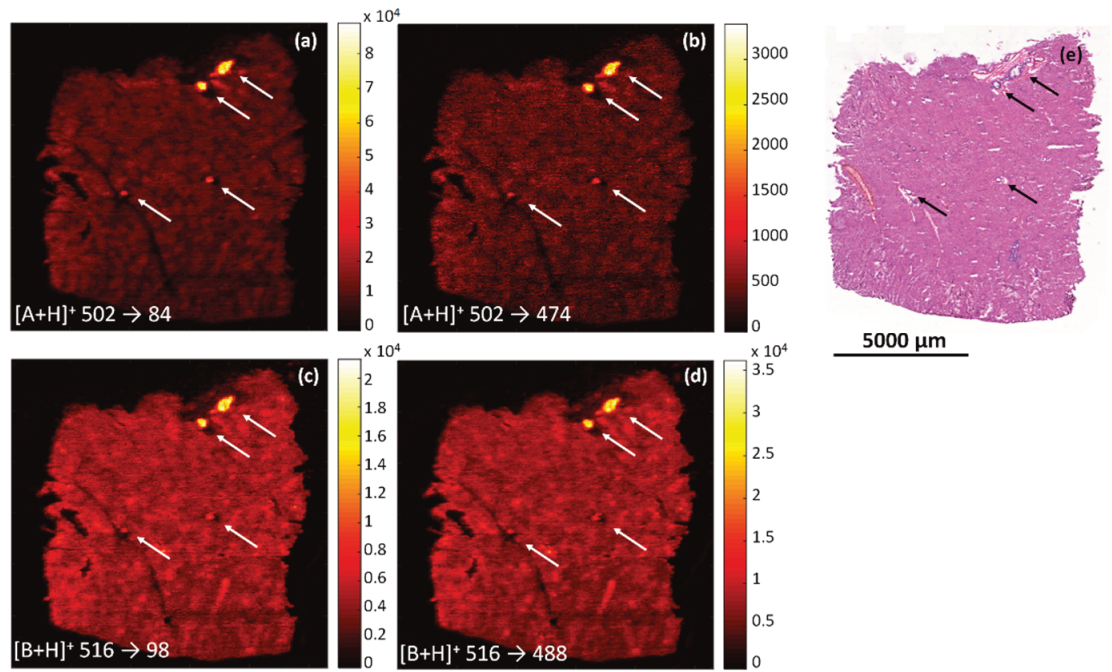

Figure 4.3 Spatial distribution of compounds A ( $a$ and $b$ ) and B ( $c$ and d) by two MRM transitions. H\&E image (e) shows tissue lesions (pointed by arrows) in the canine liver. Spatial resolution is $50 \mu \mathrm{m}$. Dwell time/MRM is $0.299 \mathrm{sec}$.

(a)

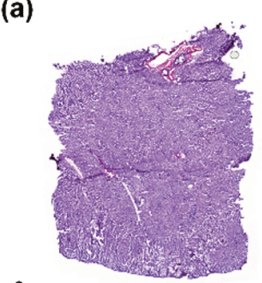

$3 \mathrm{~mm}$ (b)

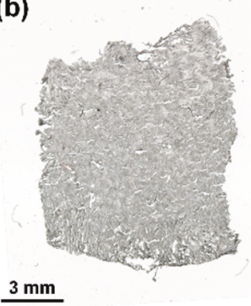

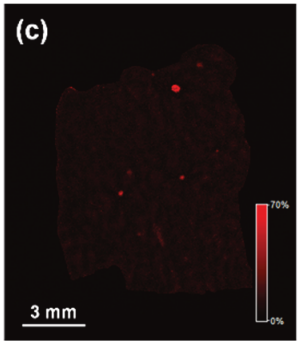

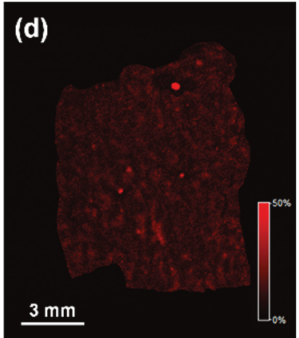

Figure 4.4 H\&E image (a), optical image (b) and MALDI-MSI images of B (c) and A (d) of same canine liver tissue. Tissue section were coated with $15 \mathrm{mg} / \mathrm{ml} \mathrm{DHB}$ in $2: 1 \mathrm{CHCl}_{3}: \mathrm{MeOH}$ with $0.2 \%$ TFA using the SunCollect sprayer (Sunchrom GmbH, Friedrichsdorf, Germany) in a series of 15 layers. The MALDI-MSI images were acquired on a Bruker RapifleX MALDI Tissuetyper instrument equipped with an Nd:YAG laser (355 nm, $10 \mathrm{kHz}$. General MS settings were as follows: mass range = m/z 400-1000; MS mode = reflectron mode; ionization mode $=$ positive ion mode; laser power $=80 \%$ with an accumulation of 200 shots; spatial resolution $=50 \times 50 \mu \mathrm{m}$. 

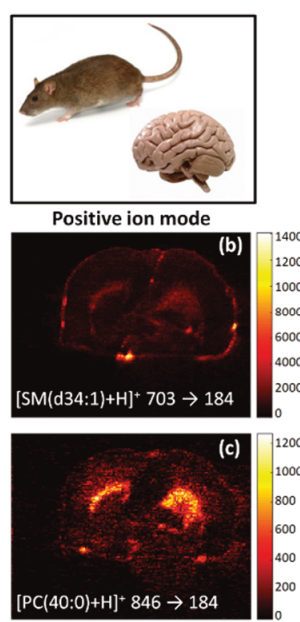

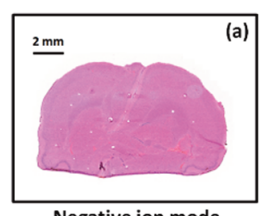

Negative ion mode
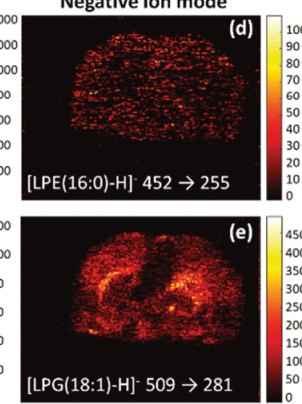

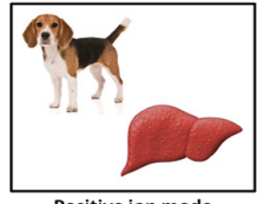

Positive ion mode
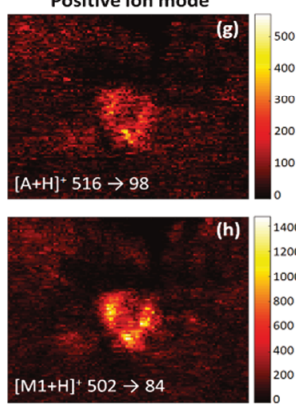

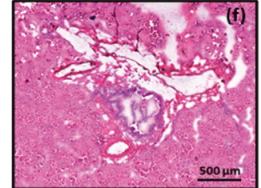

Negative ion mode
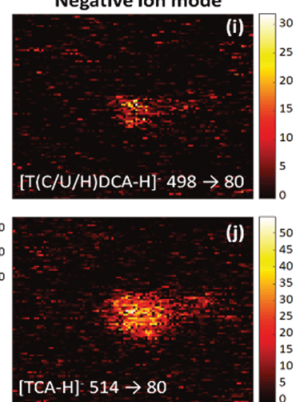

Figure 4.5 Dual polarity DESI-MRM imaging performed in one experiment. In rat brain, four different DESIMRM images of lipids are acquired of which two in +ve mode ( $b$ and $c$; dwell time $0.193 \mathrm{sec} / \mathrm{MRM}$ ) and two in -ve mode ( $d$ and e; dwell time 0.193 sec/MRM). In +ve mode, two MRM transitions target the PC head group while two different acyl chains are targeted in -ve mode. Spatial resolution is $100 \mu \mathrm{m}$. In canine liver, two MRM transitions target compound $A$ and $B$ in +ve mode ( $g$ and $h$; dwell time $0.485 \mathrm{sec} / \mathrm{MRM}$ ) and two MRM transi-tions image bile acids in -ve mode ( $i$ and $j$; dwell time 0.498 sec/MRM) that are co-localized with A and B. Spatial resolution is $30 \mu \mathrm{m}$. H\&E images of rat brain and canine liver are shown in (a and f), respectively.

\section{Dual polarity MS imaging}

In addition to the improved specificity, another benefit of using a QqQ mass spectrometer is the ability to perform fast dual polarity experiments. Figure 4.5 shows two applications of dual polarity MRM imaging: lipid imaging in rat brain and drug/metabolite imaging in canine liver. In the lipid imaging application, the images of four MRM transitions of which two transitions (Figure $4.5 \mathrm{~b}$ and $4.5 \mathrm{c}$ ) are acquired in positive ionization mode and two transitions (Figure 4.5d and 4.5e) are obtained in negative ionization mode in one experiment. Even though shown in MRM mode, the dual polarity capacity also applies to the scanning modes of the QqQ. This means that lipid classes can be evaluated based on both positive and negative charged head groups and/or acyl chains in one single experiment. In the pharmaceutical application, compound $A$ and $B$ are mapped $(4.5 \mathrm{~g}$ and $4.5 \mathrm{~h}$ ) in positive ionization mode and two bile acids are targeted (4.5i and 4.5j) in negative ionization mode. The main advantage of dual polarity MS imaging is that the information acquired originates from the same tissue section and, therefore, significantly reduces analysis time. Previous untargeted dual polarity MSI investigations ${ }^{327}$ using Orbitrap mass spectrometers have shown that polarity switching takes up to multiple seconds to reach the expected mass accuracy. The benefit of saving analysis time got lost because this one experiment takes twice as 
long. Nazari and coworker ${ }^{328}$ improved the polarity switching capability of the Orbitrap MS to a 96 ms switching time. Our QqQ MSI experiments have very limited elongation of analysis time since the polarity switching time is $15 \mathrm{~ms}$. Ellis et al. ${ }^{223}$ showed the benefit of dual polarity lipid imaging in a classification model throughout tumor tissue using MALDI-MSI. The use of dual polarity MALDI imaging has the limitation that a MALDI matrix has to be applied, which can cause potential inferences, risks delocalization, and requires sample preparation time. DESI imaging does not require an external matrix to facilitate ionization and, therefore, does not suffer from these limitations. Taylor and coworkers reported the investigation of ion suppression of olanzapine on mouse brain where DESI ionization suffered from significantly less ion suppression than MALDI ionization ${ }^{202}$. Depending on the chemical structure of the drug candidate, this makes DESI dual polarity imaging of potentially more interest for the pharmaceutical industry over MALDI dual polarity imaging. In pharmaceutical sciences, the possibility of drugs and its metabolites ionizing in different polarities is also very probable. For instance, a drug that ionizes in positive mode will ionize in negative mode after glucuronide or sulfate conjugation ${ }^{329}$. In addition, possible endogenous markers for drug candidates could also ionize best in the opposite ionization mode (e.g. bile acids for bile duct cancer drugs). This is also shown in our drug/metabolite application in which two possible drug candidates and two bile acids are images and show colocalization. The ability to map drug candidates, drug metabolites and endogenous metabolites in one experiment is crucial in further targeted imaging analyses and, therefore, DESI dual polarity imaging can play an important role.

\subsection{Conclusion}

This work demonstrates a novel targeted DESI imaging platform by using a QqQ mass analyzer combined with a targeted MS/MS-based data acquisition approach. The opportunity to perform dual polarity imaging combined with DESI is very advantageous for lipid and metabolite imaging since it does not rely on MALDI matrices. QqQ scanning modes are compared with MRM showing increased ion detection time of MRM. This opens doors for the separation of isobars and certain isomers which cannot be separated with current high mass resolving power MSI technologies. Although this targeted platform requires fragmentation knowledge the MSI field will significantly benefit from this as its applications are increasing. Our platform shows its strength in toxicology studies by selective imaging of drugs and metabolites in tissue. Spatial distribution is confirmed by monitoring the presence of pharmaceutical compounds using multiple MRM transitions of the same precursor ion. DESI-MRM imaging allows for selective imaging without the need for expensive MSI instrumentation.

MSI can suffer from lower sensitivity compared to techniques that combine mass spectrometry with separation step prior to ionization ${ }^{137}$. The use of MRM in the MS 
imaging field is very promising because improved selectivity is needed to visualize more compounds without the interference of surrounding isobars. 


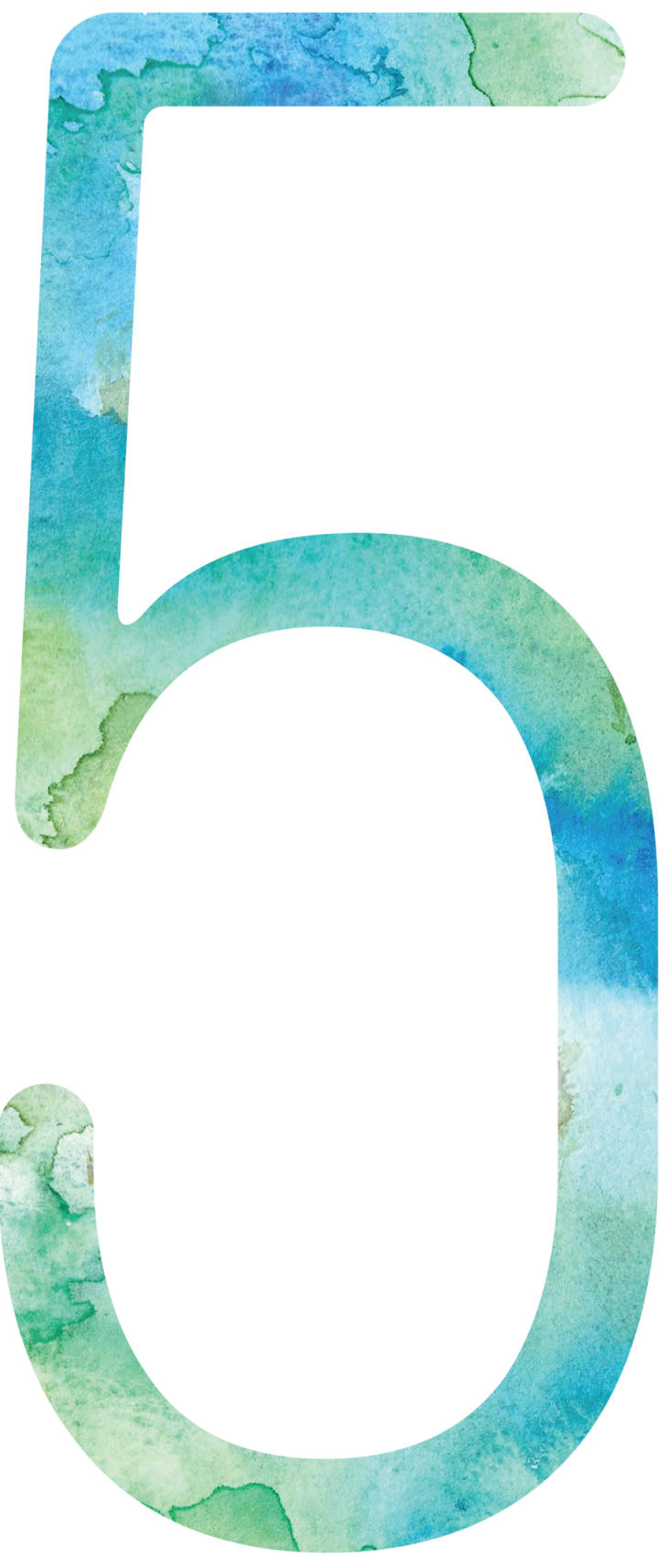




\section{MRM based Quantitative Mass Spectrometry Imaging}

Based on

Quantitative Mass Spectrometry Imaging of Drugs and Metabolites: a Multiplatform Comparison Lieke Lamont, Darya Hadavi, Brent Viehmann, Bryn Flinders, Ron M. A. Heeren, Rob J. Vreeken, and Tiffany Porta Siegel

Manuscript submitted for publication 
Mass spectrometry imaging (MSI) provides insight in the molecular distribution of a broad range of compounds and, therefore, is frequently applied in the pharmaceutical industry. Pharmacokinetic and toxicological studies deploy MSI to localize potential drugs and their metabolites in biological tissues but currently require other analytical tools to quantify these pharmaceutical compounds in the same tissues. Quantitative mass spectrometry imaging (Q-MSI) is a field with challenges due to the high biological variability in samples combined with the limited sample cleanup and separation strategies available prior to MSI. In consequence, more selectivity in MSI instruments is required. This can be provided by multiple reaction monitoring (MRM) which uses specific precursor ion-fragment ion transitions. This targeted approach is in particular suitable for pharmaceutical compounds because their molecular identity is known prior to analysis. In this work, we compared different analytical platforms to assess the performance of MRM detection compared to other MS instruments/MS modes used in a Q-MSI workflow for two drug candidates (A and B). Limit of detection (LOD), linearity, and precision and accuracy of two quality control (QC) samples were compared between MS instruments/modes. MRM mode on a triple quadrupole mass spectrometer (QqQ) provided the best overall performance with the following results for compound A and B: LOD 35.5 and $2.5 \mu \mathrm{g} / \mathrm{g}$ tissue, R2 0.97 and 0.98 linearity, relative standard deviation QC $<13.6 \%$, and $97-112 \%$ accuracy. Other MS modes resulted in LOD 6.7569.4 and 2.6-119.1 $\mu \mathrm{g} / \mathrm{g}$ tissue, R2 0.86-0.98 and 0.86-0.98 linearity, relative standard deviation QC $<19.4$ and $<37.5 \%$, and $70-356 \%$ and $64-398 \%$ accuracy for drug candidate $\mathrm{A}$ and $\mathrm{B}$, respectively. In addition, we propose an optimized 3D printed mimetic tissue model to increase the overall analytical throughput of our approach for large animal studies. The MRM imaging platform was applied as proof-of-principle for quantitative detection of drug candidates A and B in four dog livers and compared to LC-MS. The Q-MSI concentrations differed $<3.5$ times with the concentrations observed by LC-MS. Our presented MRM based Q-MSI approach provides a more robust and high throughput analytical platform due to MRM specificity combined with an optimized 3D printed mimetic tissue model. 


\subsection{Introduction}

Mass spectrometry imaging (MSI) has proven to be an invaluable tool for the pharmaceutical industry to directly localize drugs and related metabolites from tissue specimens ${ }^{83}, 142$. In the field of drug discovery and development, MSI has been applied in (amongst others) pharmacokinetic and toxicological studies to investigate whether a drug and/or metabolite accumulate in tissue. This potentially toxicological accumulation could lead to exclusion of a compound from the drug development pipeline ${ }^{141}$. Therefore, it is of utmost importance to spatially quantify the accumulation of a possible drug candidate and its metabolites. The ability to provide reliable absolute quantitative information is challenged by numerous factors. These include the lack of sample cleanup or chromatography and, as a result, occurrence of tissue ion suppression and interference of isobaric species in complex biological samples ${ }^{137,245}$.

Quantitative MSI (Q-MSI) has been a field of discussion in the past decade ${ }^{142,201,330 .}$ Researchers have investigated several approaches ${ }^{331-333}$ to improve Q-MSI performance 204,208 . Three common strategies have been applied for the addition of the calibration standards: i) the tissue extinction coefficient (TEC) model ${ }^{208}$, ii) the dilution series model ${ }^{196}$, and iii) the mimetic tissue model206. The TEC approach uses the drug standard ('pseudo' internal standard) sprayed onto a control tissue section. The TEC is a regional correction factor calculated by the intensity of the standard on tissue divided by the intensity of the standard on the glass slide. The TEC can be calculated for different regions within the tissue sample. The sample preparation is quick but the approach lacks the ability to correct for tissue ion suppression and extraction efficiency. The dilution series strategy uses calibration standards spotted onto or below a control tissue section. This way the ion suppression effects are better mimicked but the correction for extraction efficiency is still limited. Recently, the mimetic tissue model was introduced by Groseclose and coworkers ${ }^{206}$ and uses tissue homogenates spiked with calibration standards to quantify targeted compounds in the tissue section. Even though it requires more sample preparation, this strategy closely resembles the drug 'in-tissue'. Hansen et al. ${ }^{334}$ and Barry et al. ${ }^{207}$ have both compared the mimetic tissue model and the dilution series model. Much lower absolute intensities and a lower slope value suggest that the mimetic tissue model experiences more tissue ion suppression. As a result, the mimetic tissue model appropriately corrects tissue specific ion suppression effects and extraction efficiencies better than in the dilution series approach. Barry et al. recently reported a revised mimetic tissue model ${ }^{207}$ to overcome the time-consuming sample preparation. A quantitative assessment of its performance demonstrated the benefit of their approach ${ }^{335}, 336$. Nevertheless, this approach risks cross-contamination amongst calibration levels when the frozen tissue homogenate are removed from the mold. Majority of reported research uses an isotope-labelled standard for correction as the occurrence of tissue ion suppression is not only tissue/organ- 
specific but also analyte-specific. However, isotope-labelled analogues are rarely available for drug candidates under development or are extremely expensive to obtain. Several strategies and instruments are available for MSI to overcome the challenge of ion suppression due to the lack of sample cleanup. Derivatization strategies ${ }^{110}$ and washing steps, to remove competing sample matrix molecules, ${ }^{337}$ can be applied complementary to the use of an internal standard (if available). When limited sample preparation strategies are applicable, isobaric interferences can mask the detection of the analyte. High selectivity can separate these isobaric interferences in complex biological samples. This could be achieved through the use of ion mobility separation ${ }^{100}$ or high resolving power mass spectrometry combined with imaging ${ }^{317}$. Targeted MSI has also shown advantages for isobaric separation from biological tissue samples ${ }^{203,}, 320$. For example, multiple reaction monitoring (MRM) combined with MSI showed promise in several applications in drug distribution studies ${ }^{323,338}$ and endogenous metabolites ${ }^{126}$. Although the use of MRM is already standard practice in the pharmaceutical industry for decades, this is not the case for pharmaceutical MSI. The main advantages of MRM imaging are the high throughput and specific screening of multiple known analytes and a high dynamic range of the instrument ${ }^{339}$. The improved specificity (i.e. near 100\% selectivity for one specific analyte) of MRM imaging decreases the interference of background ions and leads to an improved signal-to-noise. This feature of MRM imaging could lead to enhanced performance of Q-MSI. We recently introduced a targeted imaging approach through a combination of MRM with desorption electrospray ionization (DESI) imaging ${ }^{340}$. Although the Q-MSI discussion mainly focuses on MALDI imaging, DESI has established itself as the main ambient ionization source for MSI130. DESI uses an electrospray jet to extract and map molecules directly from tissue sections ${ }^{117}$. Unlike MALDI imaging, DESI does not suffer from the signal variation caused by the application of a MALDI matrix prior to MSI analysis. The pharmaceutical industry showed increased interest in DESI imaging ${ }^{313}$ for multiple applications ${ }^{144,}, 176,186$, 314. A targeted MRM imaging platform could be of great interest for the pharmaceutical industry since the analyte of interest is known prior to analysis and reduces the analysis costs compared to high mass resolving power MSI. We investigate the potential of MRM imaging for quantitative drug imaging in the context of the pharmaceutical demand for Q-MSI technology.

Here, we evaluate different platforms to improve selectivity and sensitivity of Q-MSI. We established a multiplatform comparison among different MS detection modes and instruments, followed by a Q-MSI application of two drug candidates in dog liver. First, a mimetic tissue model was adapted to investigate the sensitivity of different platforms using two drug candidates. These samples were analyzed on three different Waters mass spectrometers compatible with the same DESI source and using the same control software: two quadrupole time-of-flight (Q-TOF) instruments (Synapt G2-Si and Xevo 
G2-XS) and a tandem quadrupole or triple quadrupole (QqQ) instrument (Xevo TQ-S micro), consisting of two quadrupoles and a nonquadrupolar collision cell ${ }^{3}$. We hypothesize that the QqQ has the best analytical performance in MRM mode due to the additional specificity. Limits of detection (LOD), linearity, accuracy and precision from low and high concentrations of quality control (QC) samples were calculated for two drug candidates, to compare the performances of the different platforms and analysis modes. As a proof-of-principle, the mass spectrometer with the best analytical performance was used to map and quantify both drug candidates in dog liver tissues. Quantitative DESI-MSI results were compared to drug levels measured by LC-MS analysis of the same samples.

\subsection{Experimental}

\section{Materials and Reagents}

ULC/MS-grade water $\left(\mathrm{H}_{2} \mathrm{O}\right)$, ULC/MS-grade methanol $(\mathrm{MeOH})$, LC/MS-grade ethanol (EtOH), LC/MS-grade xylene, and 99\% formic acid (FA) were obtained from Biosolve (Valkenswaard, NL). Gelatin was purchased from Sigma-Aldrich. Microscope glass slides were obtained from Thermo Scientific (Braunschweig, DE).

\section{Mimetic tissue model preparation and multiplatform comparison setup}

A mold was printed from VeroWhitePlus RGD835 (Stratasys, DE) using a 3D Objet30 Prime printer (Stratasys, DE). The inside of the mold was covered with a thin layer of clear nail polish (Hema, NL) to prevent adhesion by gelatin. A warm $15 \%$ gelatin solution was pipetted into the mold and cooled on ice for $30 \mathrm{~min}$ to set. In the multiplatform comparison, chicken liver (Plus supermarket, Maastricht, NL) homogenates were prepared by a mini-bead beater and $1.0 \mathrm{~mm}$ glass beads from BioSpec Products (Bartlesville, OK, USA). Chicken liver tissue was used due to the large amount of tissue necessary in the multiplatform comparison. Two drug candidates $A$ and $\mathrm{B}$ (Janssen $\mathrm{R} \& \mathrm{D}$, Beerse, $\mathrm{BE}$ ) were dissolved in $\mathrm{H}_{2} \mathrm{O}$ and $5 \mu \mathrm{l}$ were added to $100 \mathrm{mg}$ of the chicken liver prior to homogenization. Final concentrations ranged from 12.5 to $2500 \mu \mathrm{g} / \mathrm{g}$ (C1-C7 calibration levels) tissue. The gelatin block was filled with spiked tissue homogenates in a randomized order to prevent leverage, frozen at $-20^{\circ} \mathrm{C}$ and cryosectioned onto glass slides. Glass slides were stored at $-80{ }^{\circ} \mathrm{C}$ until DESI-MSI analysis. Calibration lines and quality control (QC) samples at 25 and $1250 \mu \mathrm{g} / \mathrm{g}$ level were prepared in triplicate ( $\mathrm{n}=3$ tissue homogenate preparations). 


\section{Absolute quantitation in dog liver}

Animal studies were conducted in accordance with all institutional and national guidelines for the care and use of laboratory animals. Control and dosed dog liver tissues (Beagle) were obtained from Janssen R\&D (Beerse, BE). Two drug candidates (A and B) were individually dosed at $65 \mathrm{mg} / \mathrm{kg}$ and drug candidate $A$ was also dosed at 15 $\mathrm{mg} / \mathrm{kg}$ via single oral administration. In total, four dogs were included in this proof-ofconcept experiment: two animals were dosed $65 \mathrm{mg} / \mathrm{kg}$ drug candidate $B$ (dog 1 and 2; $n=2)$, one animal was dosed $65 \mathrm{mg} / \mathrm{kg}$ drug candidate $A(n=1)$, and one animal was dosed $15 \mathrm{mg} / \mathrm{kg}$ drug candidate $A(n=1)$. All animals were sacrificed 14 days post dose. In vivo, drug B metabolizes into a demethylated metabolite identical to the structure of compound A. Partial molecular structures of both drug candidates as well as their mostlikely fragment ion, formed upon collisional activation of the protonated precursor ion, used in MRM is shown in Figure 5.1. This research presents an optimized MRM based Q-MSI approach compared to other MS modes and complemented with an MRM based proof-of-principle application. Since this research is not a biological investigation of the drug candidates but a technological comparison for Q-MSI, we believe full structural information is not required and, for confidentiality reasons, only the relevant structure is included. A calibration line and quality control $(Q C)$ samples $(n=3)$ with drug candidate $A$ and $B$ were prepared in control dog liver using the mimetic tissue model previously described. Fresh-frozen tissues and gelatin block were stored at $-80^{\circ} \mathrm{C}$ until cryosectioning. A Microtome cryostat (Thermo Scientific, Braunschweig, DE) was used to cryosection liver tissue and the gelatin block into $12 \mu \mathrm{m}$-thick slices and subsequently thaw mounted the sections onto microscope glass slides. All glass slides were stored at $-80^{\circ} \mathrm{C}$ until DESI-MSI analysis. 
<smiles>[R]c1ccc(OC2CCNCC2)cc1</smiles>

Drug A $(\mathrm{MW}=501 \mathrm{~g} / \mathrm{mol})$<smiles>[R]c1ccc(OC2CCN(C)CC2)cc1</smiles>
Drug B $(\mathrm{MW}=515 \mathrm{~g} / \mathrm{mol})$<smiles>C1=C[NH2+]CCC1</smiles>

\section{Fragment $\mathrm{A}$}

$(m / z 84)$<smiles>CN1C=CCCC1</smiles>

Fragment B

$(\mathrm{m} / \mathrm{z}$ 98)

Figure 5.1. Partial molecular structures of drug candidate A and B at, respectively, $501 \mathrm{~g} / \mathrm{mol}$ and $515 \mathrm{~g} / \mathrm{mol}$. Two most-likely fragment ions that are used in the MRM transitions are shown at $\mathrm{m} / \mathrm{z} 98$ and $\mathrm{m} / \mathrm{z} 84$.

\section{Hematoxylin and Eosin (H\&E) staining protocol}

Tissue slices used for DESI-MSI analysis were stained after completion of the MSI acquisition using a standard $\mathrm{H} \& \mathrm{E}$ staining protocol. Sections were washed in successive EtOH baths $(100 \%, 96 \%, 96 \%, 70 \%, 70 \%)$ and deionized $\mathrm{H}_{2} \mathrm{O}$ for 3 min each. Hematoxylin (Merck, Darmstadt, DE) staining was performed for 3 min followed by a gentle 3 min wash with running tap water. Eosin (Avantor Performance Materials B.V., Arnhem, NL) staining was executed for $30 \mathrm{~s}$ and washed gently with running tap water for $3 \mathrm{~min}$. The staining was finalized by an EtOH wash for $1 \mathrm{~min}$ and a xylene wash for $30 \mathrm{~s}$. The slides were covered by placing coverslips on the stained tissues using Entellan (Merck, Darmstadt, DE). Optical images were acquired using a VENTANA iScan HT scanner (Roche Diagnostics, Indianapolis, IN, USA).

\section{DESI-MSI instrumentation}

DESI-MSI analysis was performed using a DESI source (Waters, Wilmslow, UK) mounted onto three different Waters mass spectrometers: Xevo G2-XS Q-TOF, Synapt G2-Si QTOF, and Xevo TQ-S micro QqQ. DESI solvent ( $\left.\mathrm{MeOH} / \mathrm{H}_{2} \mathrm{O} / \mathrm{FA}, 98 / 2 / 0.1\right)$ was supplied to the DESI source by a Waters ACQUITY UPLC M-class binary solvent manager at 2 $\mu \mathrm{L} / \mathrm{min}$. General DESI parameters were: $\mathrm{N}_{2}$ nebulizing gas pressure $=4$ bar; spray voltage $=3-4 \mathrm{kV}$; Source temperature $=150^{\circ} \mathrm{C}$; sampling cone voltage $=70 \mathrm{~V}$; Heated custombuilt inlet capillary $=500^{\circ} \mathrm{C}$. All experiments were executed in positive ionization mode. The multiplatform comparison was performed at a pixel size of $100 \mu \mathrm{m}$ and the quantitative drug application was executed at $50 \mu \mathrm{m}$. Different MS modes were used 
in the sensitivity comparison: MS scan (all mass spectrometers), ion mobility spectrometry (IMS)-MS and IMS-MSE (Synapt Q-TOF without and with data independent acquisition, respectively), and MRM (Xevo QqQ). All Q-TOF experiments were performed in sensitivity mode. Total scan times were kept constant at $0.986 \mathrm{~s} /$ pixel. MRM dwell times were set to $0.247 \mathrm{~s} /$ pixel with in total 3 MRM transitions per acquisition: $m / z 502 \rightarrow m / z 84$ (compound $A$; CE 40V), $m / z 516 \rightarrow m / z 98$ (compound B; CE 40V), $m / z 782 \rightarrow m / z 184$ (endogenous lipid; CE 30V). All MS images were acquired using HDI Imaging (Waters, Milford, MA, USA), MassLynx version 4.1 (Waters, Milford, MA, USA), and Omni Spray 2-D version 2.0.1 (Prosolia, Indianapolis, IN, USA).

\section{Data processing and visualization}

HDI Imaging software (Waters, Wilmslow, UK) was used for visualization of MS images acquired on Xevo Q-TOF and Synapt Q-TOF and select regions of interest (ROI). We used an in-house written Matlab script (Matlab v. R2015a, MathWorks, Natick, MA, US) for data visualization and ROI selection of the MRM and MS scan data images acquired on the Xevo QqQ. Due to the absence of an isotope-labelled analog of the drug candidates, a homogeneously distributed endogenous lipid $(\mathrm{m} / \mathrm{z} 782.6)$ was used to correct for tissue matrix effects. From each ROI, the extracted MS spectra were summed and different peak ratios (analyte-to-lipid) were calculated to build the calibration lines. QuPath software (v0.1.2, The Queen's University of Belfast, Northern Ireland) was used to select the ROIs from the H\&E images and calculate the amount of cells present in the ROI. The H\&E images were overlaid with the MSI images by an in-house developed Matlab script and overlapping pixels were extracted. The drug candidate/lipid ratio was calculated after correction for the number of cells detected in the ROI. This workflow can be found in the Figure $5.2^{90}$. All calibration lines are constructed according to Eurachem guidelines ${ }^{341}$. When a calibration standard concentration was $\angle L O D$, this point was not included in the calibration line. 


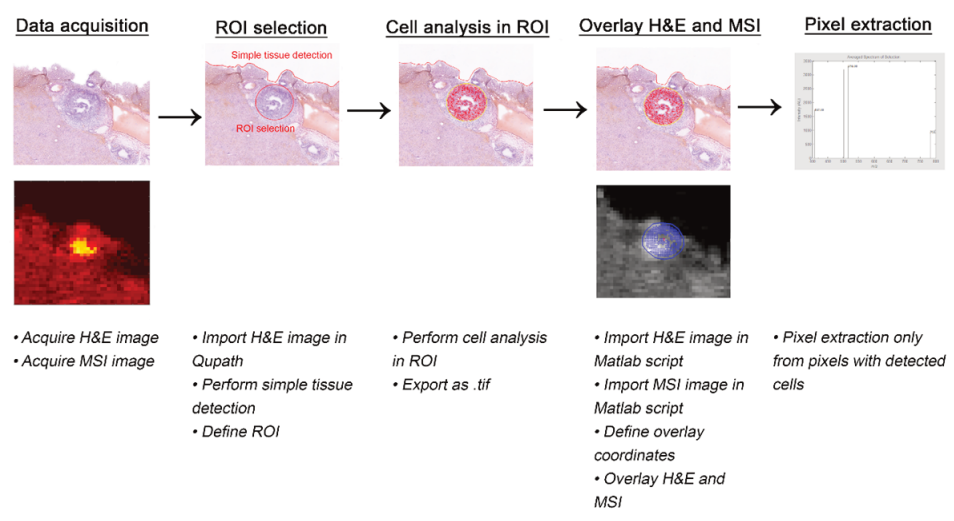

Figure 5.2 Data analysis workflow consists of a 5-step procedure. Step 1: MSI and H\&E images are acquired. Step 2: ROI selection in QuPath. Step 3: Cell analysis in QuPath to detect cells. Step 4: Overlay H\&E image (with detected cells marked) and MSI image in an in-house written Matlab script. Step 5: MSI pixel selection and extraction in in-house written Matlab script. From these MS spectra, intensity ratios between the drug candidates and endogenous lipid are used.

\subsection{Results and discussion}

\section{Optimization of mimetic tissue model}

The 'in-tissue' approach requires a very laborious process due to the homogenization step $^{342}$. Here, we optimized the sample preparation protocol of a mimetic tissue model for high throughput Q-MSI purposes by designing a 3D printed mold (Figure 5.3), used to prepare a gelatin block with a tissue mimetic array. 

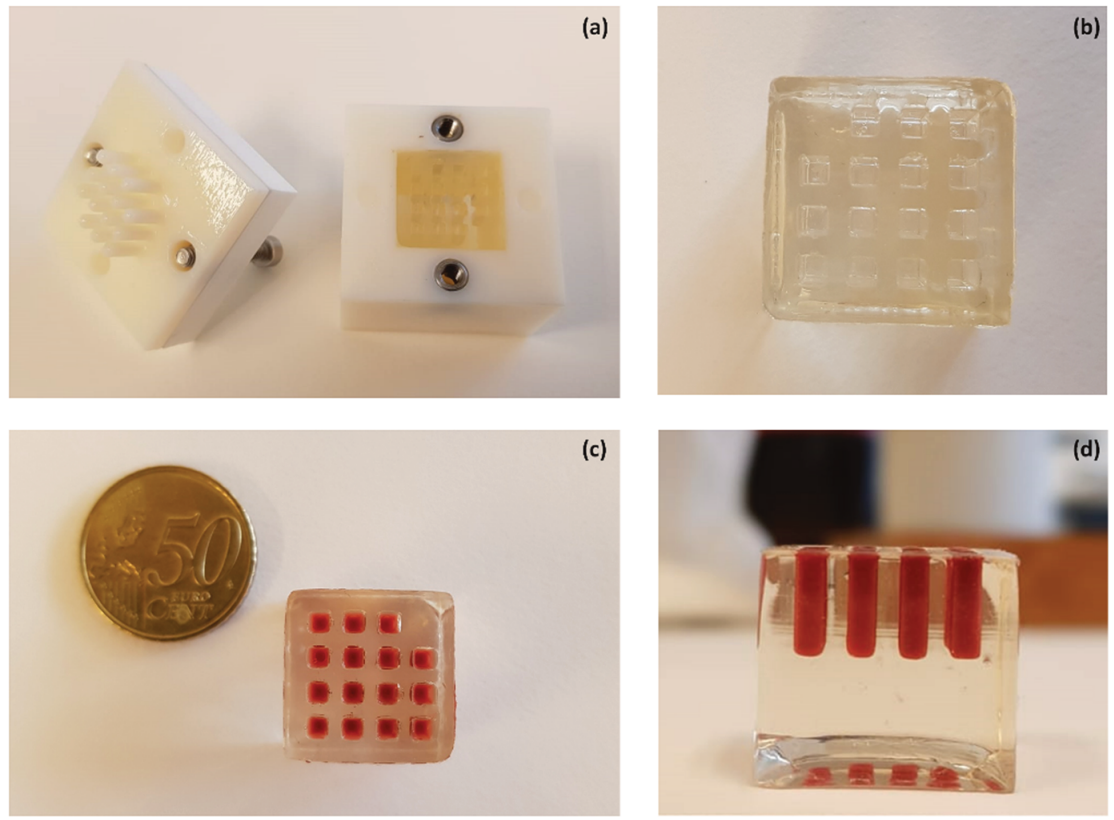

Figure $\mathbf{5 . 3}$ Overview of the mimetic tissue model. Presentation of the 3D printed mold with two sets of screws and 15 pillars in the lid (a). Prepared gelatin block after hardening on ice (b). The gelatin block filled with spiked tissue homogenate ( $\mathrm{c}$ and $\mathrm{d}$ ) before storage at -80C.

This 3D printed mold (Figure 5.3a) was designed with a lid containing 15 squared pillars with $3 \times 3 \times 8 \mathrm{~mm}$ dimensions $(\mathrm{L} \times \mathrm{W} \times \mathrm{H})$ screwed onto the outer walls of the mold. A warm $15 \%$ gelatin solution was pipetted into the mold. Gelatin is often used for tissue embedding to assist in cryosectioning fragile or small tissues and is demonstrated to not interfere in the mass spectrum (unlike OCT embedding) ${ }^{343,344}$. After the gelatin was hardened on ice, the two other screws were used to gently remove the lid from the gelatin block. The distance between the pillars of the mold was set at $2 \mathrm{~mm}$ to prevent cross-contamination between pillars due to diffusion of molecules from the tissue homogenate into the gelatin. This diffusion was investigated for both drug candidates and the endogenous lipid used for signal normalization (Figure 5.4). 
Compound A

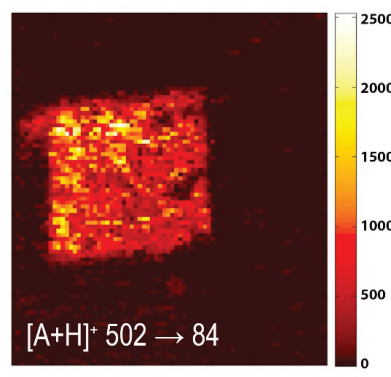

Compound B

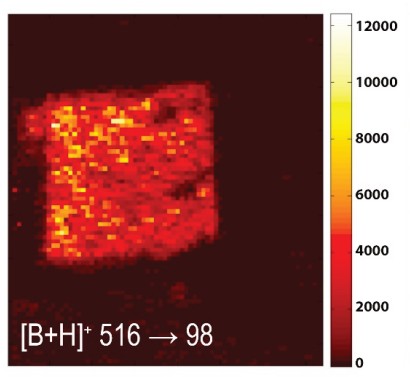

Lipid

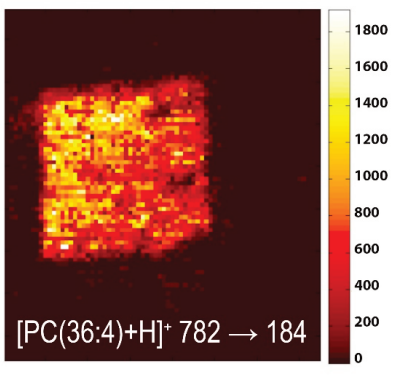

Figure 5.4 Diffusion of drug candidates into gelatin is shown by DESI-MRM images of a tissue homogenate pillar for drug candidate A, drug candidate B, and the endogenous lipid (used as a correction factor). The MRM images were acquired in positive ion mode at a spatial resolution of 50 $\mu \mathrm{m}$. Dwell times were $0.247 \mathrm{~s} / \mathrm{pixel}$.

We noticed minimal diffusion and no cross-contamination of the drug candidates and endogenous lipid. The total dimension of the gelatin block was of $20 \times 20 \mathrm{~mm}^{2}(\mathrm{~L} \times \mathrm{W}$; Figure $5.3 \mathrm{~b}$ and $5.3 \mathrm{c}$ ) to fit on a microscope glass slide and to use the additional space for the sample tissue section. The 15 pillars were arranged with one empty corner to keep the orientation clear, which is important after addition of different tissue concentration levels. The volume of the spiked calibration standard was kept 5\% (w/w) of the total tissue weight to limit the change in tissue density. The highly viscous tissue homogenate was aspirated using a disposable syringe to avoid air bubbles in the homogenates and dispensed into the empty pillar using both the syringe and a needle, which resulted in a filled gelatin block (Figure 5.3d). The design of our mimetic tissue model allows for high throughput application due to the large amount of sections that can be obtained from one gelatin block. This is of particular interest for the pharmaceutical industry when performing large scale drug studies. Figure 5.5 shows the H\&E images of liver tissue before (Figure 5.5a) and after (Figure 5.5b) homogenization. 


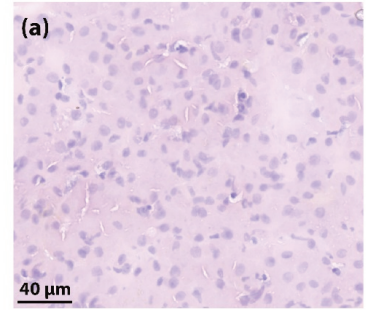

(b)

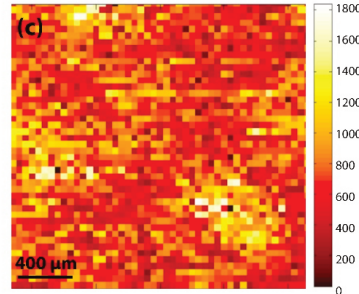

$40 \mu \mathrm{m}$
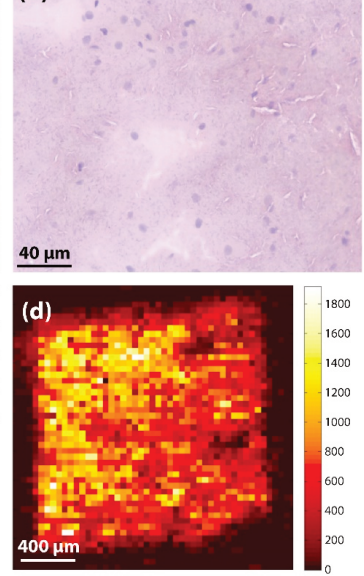

Figure 5.5 H\&E stained images from liver tissue (a) and liver tissue homogenates (b). MRM images of $m / z 782$ are shown from liver tissue (c) and liver tissue homogenates (d). Pixel-to-pixel variation of $\mathrm{m} / 2782$ in liver tissue (c) and liver tissue homogenates (d) is $<21 \%$ RSD and $<24 \%$ RSD for $n=30$ pixels, respectively.

The morphology of the tissue was lost due to the homogenization step. The addition of $5 \%$ of solution containing the spiked standard and the homogenization process itself disrupted the cells, which leaded to a lower density of intact cells and nuclei. These tissue homogenates were used to mimic the sample tissue and, therefore, simulate an average tissue ion suppression (derived from endogenous cellular compounds) as occurs in actual liver tissue sections ${ }^{334,345}$. In addition, we compared the distribution of the endogenous lipid ( $\mathrm{m} / \mathrm{z} 782$ ) before (Figure 5.5c) and after (Figure 5.5d) homogenization and we confirm that in both cases the lipid intensity and distribution are similar despite cellular disruption caused by the mechanical homogenization process. A mimetic tissue model is challenging to cryosection due to the fragility of frozen tissue homogenate. Inhomogeneity in the cryosectioned calibration line is corrected for by the use of the endogenous lipid unlike other approaches that use a sprayed isotope-labelled standard on top of the mimetic tissue model.

\section{Multiplatform comparison}

The comparison of different mass spectrometers for DESI-MSI of both drug candidates in tissue is shown in Table 5.1. For each of the MS modes, the limit of detection (LOD), linearity ( $\left.\mathrm{R}^{2}\right)$, QC precision (RSD), and QC accuracy (\%) are reported for drug candidates $A$ and $B$. In this paragraph, the results obtained in the multiplatform comparison are compared to the guideline for bioanalytical method validation as defined by the European Medicine Agency $(E M A)^{346}$. While these guidelines are set for 
chromatographic analytical tools applied in toxicological studies, most validation parameter guidelines are equally applicable to Q-MSI data. The EMA guidelines are used as a point of reference for Q-MSI data and not as a full validation protocol in the multiplatform comparison.

Table 5.1 Multiplatform comparison of drug candidate A and B

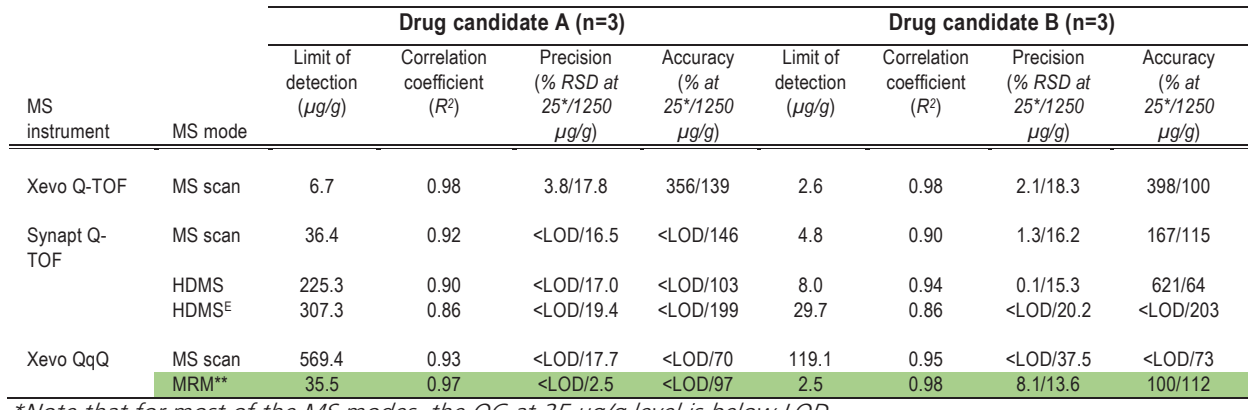

*Note that for most of the MS modes, the QC at $25 \mu \mathrm{g} / \mathrm{g}$ level is below $L O D$.

** The best instrument/mode is highlighted in green.

Sensitivity. After MSI acquisition of the calibration lines in triplicate, LOD values ${ }^{347}$ were calculated by multiplying the standard deviation (SD) of the blank intensity ratios obtained by 3 . This intensity ratio was calculated into LOD concentration levels in $\mu \mathrm{g} / \mathrm{g}$ tissue by using the slope of the calibration line:

$$
L O D=\frac{S D_{\text {blank }\left(I_{\text {drug candidate }} / I_{\text {lipid }}\right)} * 3}{\text { slope }}
$$

The Xevo Q-TOF is a very fast and sensitive instrument and frequently used in combination with DESI in MS scan mode. When comparing the LOD values with those obtained in the three scanning modes on the Synapt Q-TOF, the MS scan on the Xevo Q-TOF is the most sensitive scanning mode for both drug candidates. We investigated all three scanning modes available on the Synapt Q-TOF and all modes showed similar trend for both compounds with MS scan mode being the most sensitive. The application of ion mobility separation is known to improve selectivity due to collisions between the ions and gas molecules and, therefore, eliminates more background ions than analyte ions and enhance $\mathrm{S} / \mathrm{N}$ ratios ${ }^{55}$. However, due to these ion-neutral collisions, total ion transmission decreases significantly ${ }^{348}$. For our two drug candidates, we conclude that IMS-MSE is the least sensitive mode on the Synapt Q-TOF. MS scan mode on the Xevo QqQ gave high LOD value for compound $A$ and $B$. The LOD values reported a strong sensitivity gain in MRM mode: 50-fold for compound $B$ and a mere 20-fold for compound A. Compound A was imaged most sensitive in MS scan mode on the Xevo 
Q-TOF. In summary, distinct specificity enhancement was obtained for compound B in MRM mode versus MS scanning modes. This sensitivity gain by using MRM mode seems to be much larger for compound $B$ than for compound $A$. The explanation for a poorer sensitivity for compound $A$ in MRM mode versus compound $B$ in MRM mode could be a result of different fragmentation efficiencies. During optimization of the MRM transition, one is always searching for the most abundant fragment ion. However, this is not always a guarantee for the best $\mathrm{S} / \mathrm{N}$ ratio for this transition. Therefore, the most abundant fragment ion may not provide the same sensitivity for both compounds. In other words, if the selectivity gain (a higher $\mathrm{S} / \mathrm{N}$ ratio due to the observed background) of the MRM transition for compound $\mathrm{B}(\mathrm{m} / \mathrm{z} 98)$ is significantly higher than the $\mathrm{S} / \mathrm{N}$ of the MRM transition for compound $A(m / z 84)$, then this will result in different detection limits.

Linearity. For both compounds A and B, the best linearity was observed on the Xevo QTOF in MS scan mode $\left(R^{2}>0.98\right.$, table 5.1$)$. The significantly poorer correlation was obtained on the Synapt Q-TOF in all modes ( $R^{2}$ 0.86-0.94). On the Xevo QqQ, the correlation coefficient was poor in MS scan mode ( $\left.R^{2} 0.93\right)$ and improved in MRM mode $\left(R^{2}\right.$ 0.97) for compound $A$. For compound $B$, linearity values acquired on the Xevo QqQ $\left(R^{2}>0.95\right)$ report improvement compared to the Synapt Q-TOF and is comparable to the Xevo Q-TOF. Variability in calibration lines could be limited by including more levels per calibration line, for example $\geq 10$ calibration levels (instead of $\geq 7$ levels for chromatographic approaches).

Precision and accuracy. Spiked QC samples were included in triplicate in the experimental setup to investigate the performance of our Q-MSI workflow. QC samples were prepared at two concentration levels, i.e. 25 and $1250 \mu \mathrm{g} / \mathrm{g}$ tissue. Precision was calculated by relative standard deviations (RSD) based on $n=3$ replicates. For some MS modes, the QC samples at $25 \mu \mathrm{g} / \mathrm{g}$ were below the observed LOD values and not reported in Table 5.1. Precision should be $\pm 15 \%$ ( $\pm 20 \%$ below lower limit of quantification (LLOQ); defined as $\mathrm{SD}_{\text {blank }}$ * 5 / slope) according to the $\mathrm{EMA}^{346}$. When we compare the precision levels obtained from the different MS modes, the MRM mode is the only MS mode that meets the EMA requirements for both drug candidates. With regards to accuracy, EMA guidelines at analyte concentrations $\geq 0.1 \mu \mathrm{g} / \mathrm{g}$ is $-20 \%$ to $+10 \%{ }^{346}$. In our multiplatform comparison, no MS mode meets the EMA guidelines for both drug candidates, however, MRM mode is the closest in analytical performance to the EMA standard. The applicability of EMA guidelines to Q-MSI data is debatable due to the higher variability in Q-MSI approaches than in chromatographic strategies, which include the use of internal standards. More QC replicates in the Q-MSI experimental design could be considered to meet EMA guidelines, for example $n \geq 5$, instead of $n=3$ as used in our experiments. 
Overall, one could argue that compound B showed a significantly lower LOD value than compound A in MRM mode. The sensitivity might be better in MS scan mode (Xevo QTOF), however, the linearity and the precision and accuracy of high and low QC samples show the best analytical performance in MRM imaging mode compared to all MS scanning modes for both drug candidates. Therefore, we selected the MRM imaging mode for quantitative analysis of the drugs in dog liver samples.

\section{Absolute quantitation of drug candidate compounds in dog livers}

Q-MSI based on our optimized mimetic tissue model allows for high throughput screening in drug studies that comprise multiple animals at multiple time points. We demonstrate this Q-MSI drug application as a proof-of-concept based on four different dog liver samples. These samples were obtained from a drug development study of two possible drug candidates (compound $A$ and $B$ ) and were analyzed with DESI-MRM. Dogs 1 and 2 are dosed $65 \mathrm{mg} / \mathrm{kg} \mathrm{B}$, dog 3 is dosed $65 \mathrm{mg} / \mathrm{kg} \mathrm{A}$, and dog 4 is dosed 15 $\mathrm{mg} / \mathrm{kg}$ A for this experiment.
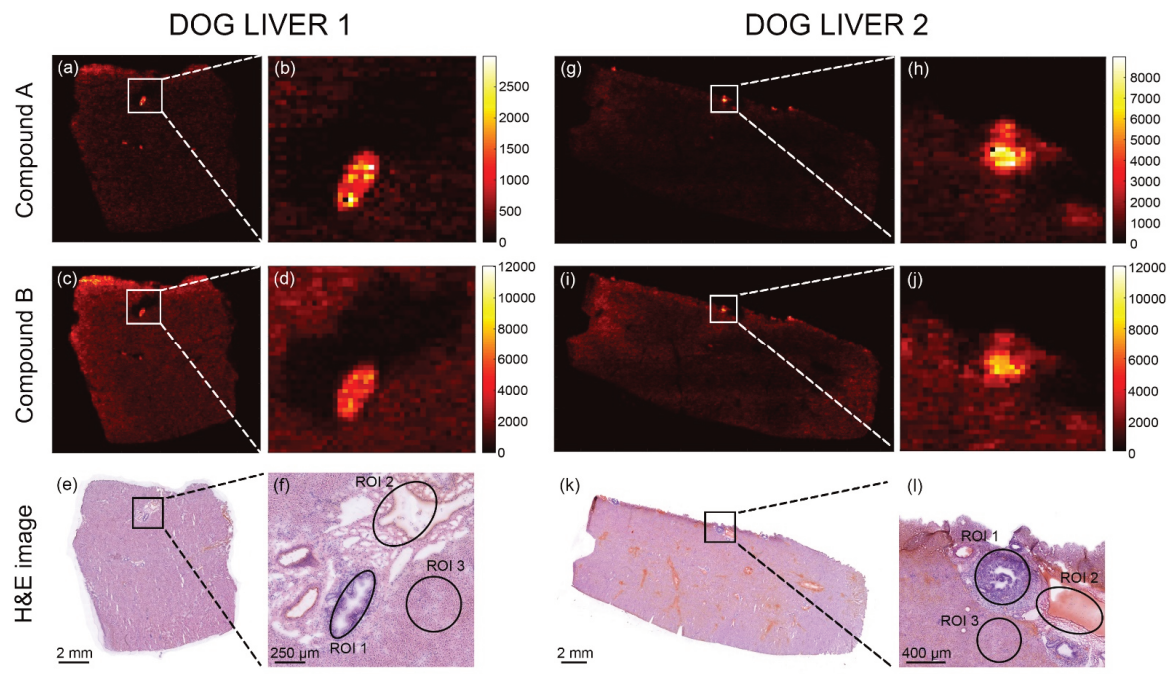

Figure 5.6 DESI-MRM images obtained from livers of dog 1 and 2 (dosed with drug candidate B at $65 \mathrm{mg} / \mathrm{kg}$ ). For dog liver 1, dealkylated metabolite image (a and b) and drug candidate B (c and d). Corresponding H\&E images (e and f) show the tissue lesion and define the three selected ROIs. In addition for dog liver 2, dealkylated metabolite image ( $g$ and $h$ ) and drug candidate B ( $\mathrm{i}$ and $\mathrm{j}$ ). Corresponding H\&E images ( $k$ and $I)$ show the tissue lesion and define the three selected ROIs.

DESI-MSI imaging of dog liver samples. Figure 5.6 displays the DESI-MRM image data obtained from sections of dog liver 1 and 2 for compound B (Figure 5.6c and 5.6i). In both dog liver sections, an accumulation of compound B in the bile duct (zoom in Figure 
5.6d and 5.6j) was noticed. The dealkylated metabolite (compound A), also detected in dog liver 1 and 2 (Figure 5.6a and 5.6g), showed the same accumulation location (zoom in Figure 5.6b and 5.6h) as drug candidate B. A detailed examination by an experienced pathologist of the H\&E stained tissue sections showed that both drug candidates caused histological lesions in the dog livers (Figure 5.6e and $5.6 f$ for dog liver 1; and Figure $5.6 \mathrm{k}$ and $5.6 \mathrm{l}$ for dog liver 2). Figure 5.7 presents the MRM compound images obtained from dog liver 3 (Figure 5.7a and 5.7b) and dog liver 4 (Figure 5.7e and 5.7f) for drug candidate A. The MRM images of the distribution of compound A in dog liver 3 and 4 , where the actual compound $A$ is directly dosed to the dogs, did not show the same accumulative pattern in "hot-spots" as occurred in dog liver 1 and 2. Q-MSI data is necessary to explain this observational difference between the two sets of dog livers.

DOG LIVER 3
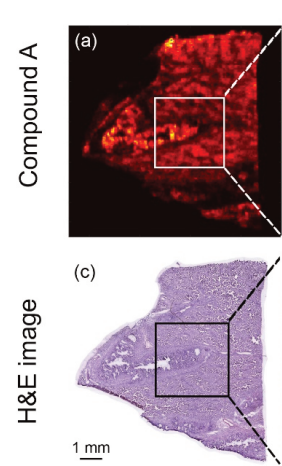
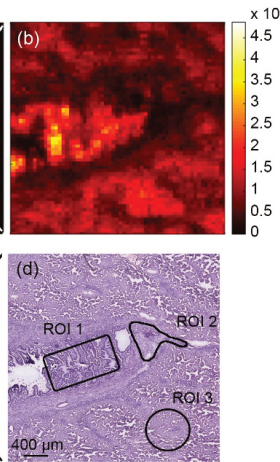

DOG LIVER 4
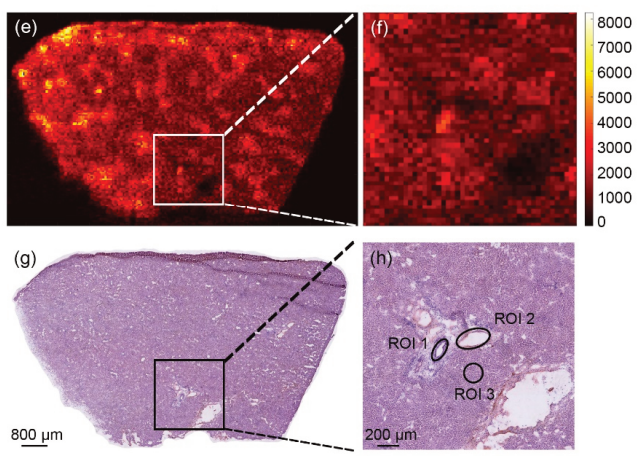

Figure 5.7 DESI-MRM images obtained from dog livers 3 and 4 of which the animals were dosed drug candidate A at $65 \mathrm{mg} / \mathrm{kg}$ and $15 \mathrm{mg} / \mathrm{kg}$, respectively. For dog liver 3, drug candidate images (a and b) are shown. Corresponding H\&E images ( $c$ and d) show the tissue lesion and define the three selected ROls. For dog liver 4, drug candidate images (e and f) are shown. Corresponding H\&E images ( $g$ and $\mathrm{h}$ ) show the tissue lesion and define the three selected ROls

From pixels to Q-MSI. For the quantitative calculations in the different dog livers, three different regions were highlighted (Figure 5.6f, 5.6l, 5.7d and 5.7h) and corresponding spectra extracted from the MSI data: $R O / 1$ ) tissue lesions in the bile duct and bile duct wall; $R O / 2)$ the connective tissue with part of the blood vessel; $R O / 3$ ) the liver parenchyma ${ }^{349}$. In each dog liver tissue, ROI 1, ROI 2 and ROI 3 were extracted in triplicate $(n=3)$. Their H\&E based regions can be found in Figure 5.8. 
Dog liver 1

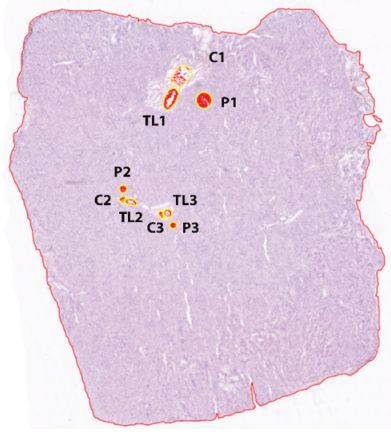

Dog liver 3

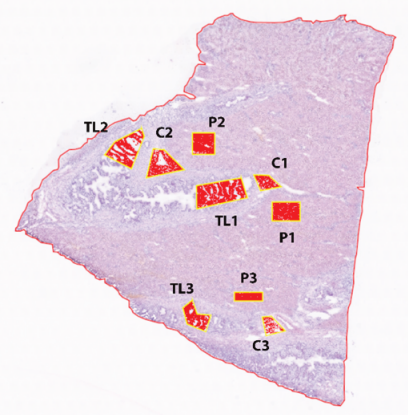

Dog liver 2

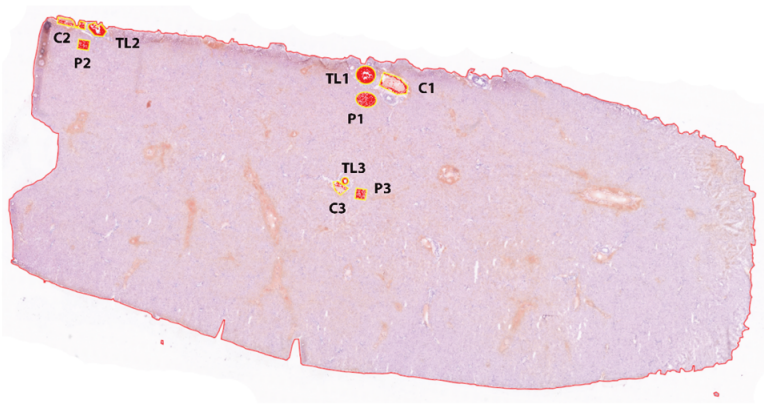

Dog liver 4

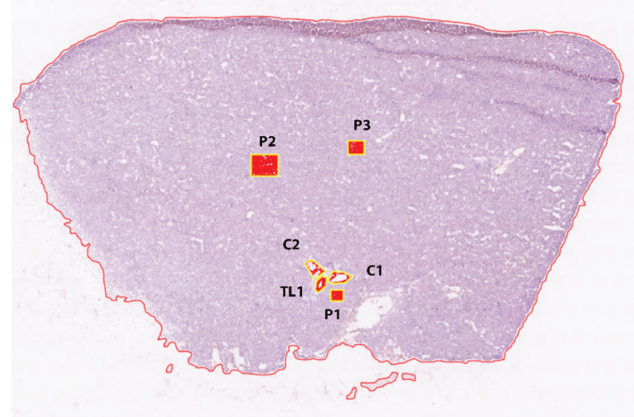

Figure 5.8 ROI selection in four dog liver tissues in their H\&E images (Qupath software). For each dog liver tissue, the tissue is annotated by a pathologist and classified as tissue lesion (TL), connective tissue (C), and parenchyma (P). Where possible for each dog liver, ROls of all tissue types are extracted in triplicate (f.e. TL1, TL2, and TL3). These H\&E images were overlaid with the MSI images as described in Figure 5.1.

Some of these extracted ROIs were marked in the H\&E images in Figures 5.6f, 5.6I, 5.7d and $5.7 \mathrm{~h}$ for dog livers $1,2,3$, and 4, respectively. Tissue ion suppression correction is necessary for more accurate quantitative results ${ }^{202}$. Due to the absence of an isotopelabelled analog of the drug candidates, an endogenous lipid $(\mathrm{m} / \mathrm{z} 782$ ) was used as correction factor. The endogenous lipid used for correction is homogeneously distributed throughout the tissue homogenates but not throughout the dog liver and, therefore, is not an ideal correction factor. The extraction of pixels that exclusively contain this endogenous lipid was guided by the regional cell count in the dog liver ${ }^{90}$. This workflow is shown in Figure 5.2. 


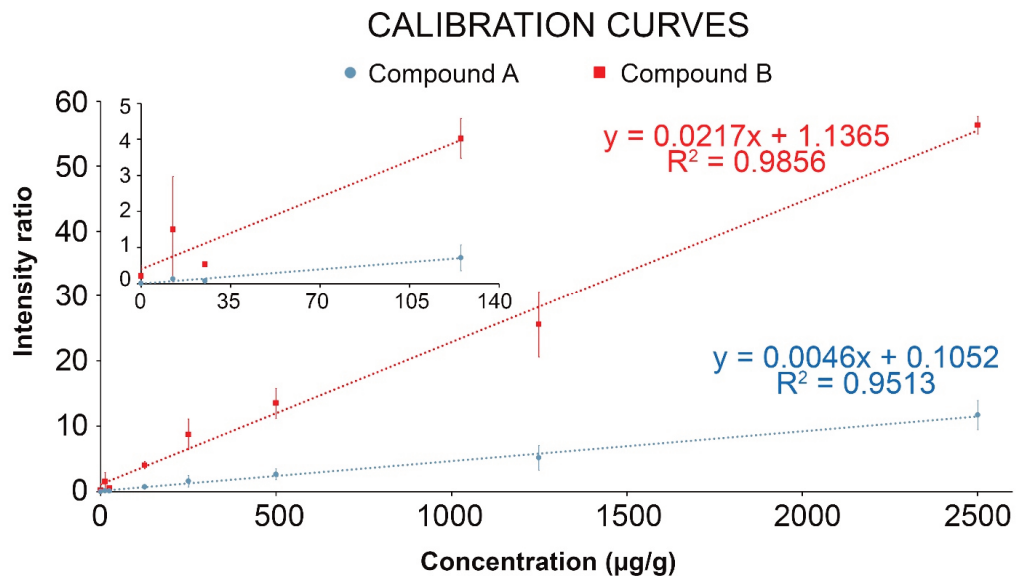

Figure 5.9 Calibration curves ( $n=2$ ) of drug candidate A (blue) and B (red) in control dog liver that were used to quantify the three ROIs in the four dog liver tissues. A separate plot depicts the lower range of the calibration curves. Error bars shows the standard deviation of the intensity ratios for each calibration level.

Figure 5.9 depicts the calibration line for both drug candidates to obtain the quantitative results in the three ROIs. These calibration lines were constructed in control dog liver with the same linear range as used in the multiplatform comparison (12.5$2500 \mu \mathrm{g} / \mathrm{g}$ ) which include the targeted concentration range. With correlation coefficients $R^{2}>0.95$, linear calibration lines were constructed and used for quantification of the three ROls. For drug candidate A, precision levels of QC samples $(\mathrm{n}=3)$ were $11 \%$ at $25 \mu \mathrm{g} / \mathrm{g}$ and $14 \%$ at $1250 \mu \mathrm{g} / \mathrm{g}$ and the accuracy was determined at $91 \%$ at $25 \mu \mathrm{g} / \mathrm{g}$ and $94 \%$ at $1250 \mu \mathrm{g} / \mathrm{g}$. For drug candidate $\mathrm{B}$, the calculated precision was $12 \%$ at $25 \mu \mathrm{g} / \mathrm{g}$ and $12 \%$ at $1250 \mu \mathrm{g} / \mathrm{g}$. Accuracy was established $94 \%$ at $25 \mu \mathrm{g} / \mathrm{g}$ and $93 \%$ at $1250 \mu \mathrm{g} / \mathrm{g}$. The final absolute concentrations quantified in the ROls are listed in Table 5.2 . 
Table 5.2 Quantification of drug candidates A and B in three ROIs in four dog liver tissues

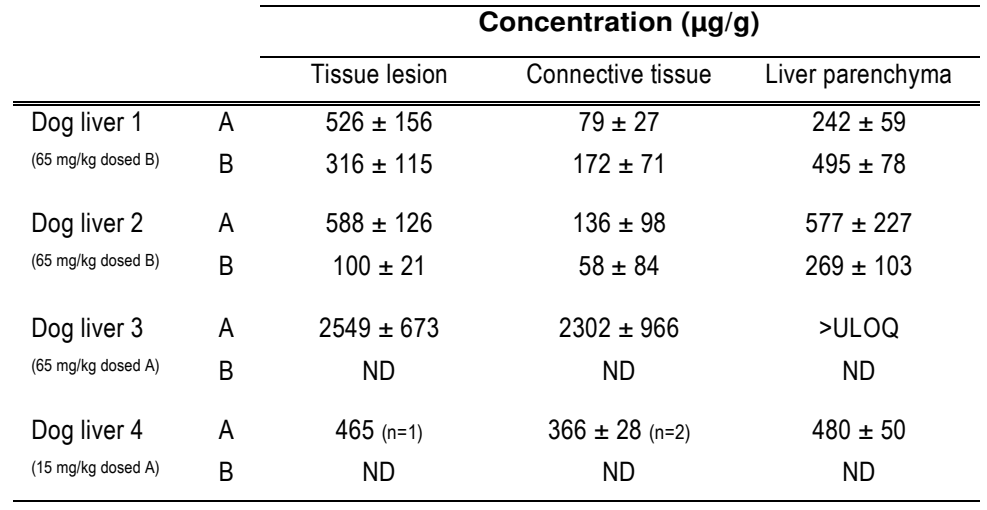

In line with the observations from the MRM images, the 'hot spot' (e.g. tissue lesion) showed a significantly higher concentration for compound A (metabolite) than in the connective tissue/blood vessel (6.6 times higher) and liver parenchyma (2.2 times higher) for dog liver 1. However, this was not the case for dog liver 2 where tissue lesions and liver parenchyma resulted in similar concentrations (lesion 1.02 times higher than parenchyma). Considering that compound $A$ is metabolized from drug candidate $B$, the concentrations observed in the tissue lesions are lower than in liver parenchyma for dog liver 1 (1.6 times lower) and dog liver 2 (2.7 times lower). It is clear from the data that for both dog liver 1 and 2, connective tissue/blood vessel resulted in significantly lower concentrations. Considering that dog liver 1 and 2 are biological replicates, a similar trend was observed amongst the three ROls. A clear difference was observed in the metabolism of the dogs, in which dog 2 metabolized more of the drug candidate (B) into its metabolite (A) than dog 1. The connective tissue/blood vessel and liver parenchyma particularly showed a higher metabolic rate, which confirms the need for multiple animals in large-scale drug studies. The absolute concentration levels of dog liver 3 and 4 showed a factor difference of $>5$ for tissue lesion (2549 and 465, respectively; factor 5.5) and connective tissue/blood vessel (2302 and 366, respectively; 6.3) which can potentially be explained by the dosage difference between dog liver 3 and 4 (factor 4.3). The observation that dog liver 3 and 4 showed minimal 'hot spots' of compound A in the MRM images is confirmed by the quantitative MSI data. All three ROls yielded similar concentrations. In addition, the differences in absolute concentrations detected in the different tissue regions stresses the need for spatial quantitative information that can explain the distribution of drugs and their metabolites in toxicological studies. Regional laser capture microdissection of tissue followed by LCMS can provide absolute quantification in tissue but is an extremely laborious approach 
when performed on multiple animals at different time points ${ }^{350,351}$. Using our mimetic tissue model allows spatial quantitative screening of drugs and metabolites in largescale toxicology studies without being too time consuming.

LC-MS comparison. The use of four dog livers from this pharmaceutical study allowed us to compare quantitative DESI-MRM data with earlier obtained LC-MS data. The comparison of Q-MSI data with LC-MS data of the same tissue is challenging because of lacking spatial information from the tissue homogenates analyzed by LC-MS. Barry and coworker discussed the legitimacy of LC-MS validation of Q-MSI based on three case studies ${ }^{336}$. Only if the MSI image shows a homogenous distribution, LC-MS data is comparable to Q-MSI data. Because our LC-MS data was obtained from assays that homogenize the whole liver tissue, as a consequence, we only compare the quantified MRM data of the liver parenchyma with the LC-MS data since the contribution of the parenchyma to homogenized tissue is by far the largest. Three different regions of interest were extracted from the liver parenchyma $(n=3)$. We only report concentration ranges of the LC-MS data from the dog livers due to confidentiality of the drug candidates. LC-MS concentrations for compound A and B obtained in dog liver 1 were $\sim 150-220 \mu \mathrm{g} / \mathrm{g}$ and $\sim 120-250 \mu \mathrm{g} / \mathrm{g}$ in dog liver 2. Drug concentrations of compound A found in dog liver 3 were above the upper limit of quantification (<ULOQ) and in dog liver 4 were $\sim 1550-1650 \mu \mathrm{g} / \mathrm{g}$. A comparison between quantitative LC-MS and quantitative DESI-MRM imaging demonstrated a difference in absolute concentrations of $<3.5$ times. Tissue heterogeneity, local information which is lost in LC-MS of tissue homogenates, influences the regional signal by the occurrence of tissue ion suppression. The ideal strategy to correct for this tissue ion suppression, is the use of an isotope-labelled analogue of the drug candidate. In addition, the contribution of the liver parenchyma to a tissue homogenate of the same liver sample is the largest but is not $100 \%$. Therefore, the comparison of LC-MS of homogenates and Q-MSI of liver parenchyma could be inadequate. LC-MS and regional sampling, such as LESA ${ }^{137}$ or laser capture microdissection ${ }^{350}$, could provide more agreement between Q-MSI and LC-MS as the distribution in tissue is the starting point.

\subsection{Conclusions}

This work reports an optimized design for mimetic tissue model followed by an analytical assessment of its performance and a proof-of-concept drug application in QMSI. Our optimized protocol fits into an 'in-tissue' approach and allows Q-MSI analysis of the sample tissue on the same glass slide. The large number of sections that can be obtained from one gelatin block can be used for large-scale drug studies. In addition to this mimetic tissue model, MRM imaging has been investigated in comparison to different MS modes. After evaluation of multiple MS modes, MRM imaging has 
enhanced the analytical performance of Q-MSI. This is a consequence of the improved specificity obtained from MRM imaging and, therefore, can visualize more targeted analytes without the isobaric interference of background compounds. As a proof-ofconcept, Q-MSI drug levels were obtained from four different dog livers and compared with quantitative LC-MS. Although more animals are needed to confirm the obtained concentration levels, significant concentration differences were observed between tissue lesions, connective tissue and blood vessels, and liver parenchyma of the two animals (dog 1 and 2) that were dosed $65 \mathrm{mg} / \mathrm{kg}$ drug candidate B. The two animals (dog 3 and 4) that were dosed 65 and $15 \mathrm{mg} / \mathrm{kg}$ drug candidate A, respectively, did not show this accumulative behaviour of the drug candidate in the tissue lesion. The comparison with LC-MS data revealed a concentration difference of $<3.5$ times between quantitative LC-MS and quantitative MRM imaging for liver parenchyma. The analytical validation of Q-MSI is still challenging and needs to be compared with LCMS. Our developments contribute to a more robust Q-MSI workflow for drug imaging: an adapted mimetic tissue model for high throughput toxicological studies and a more sensitive and selective MRM detection to improve biological variability. 


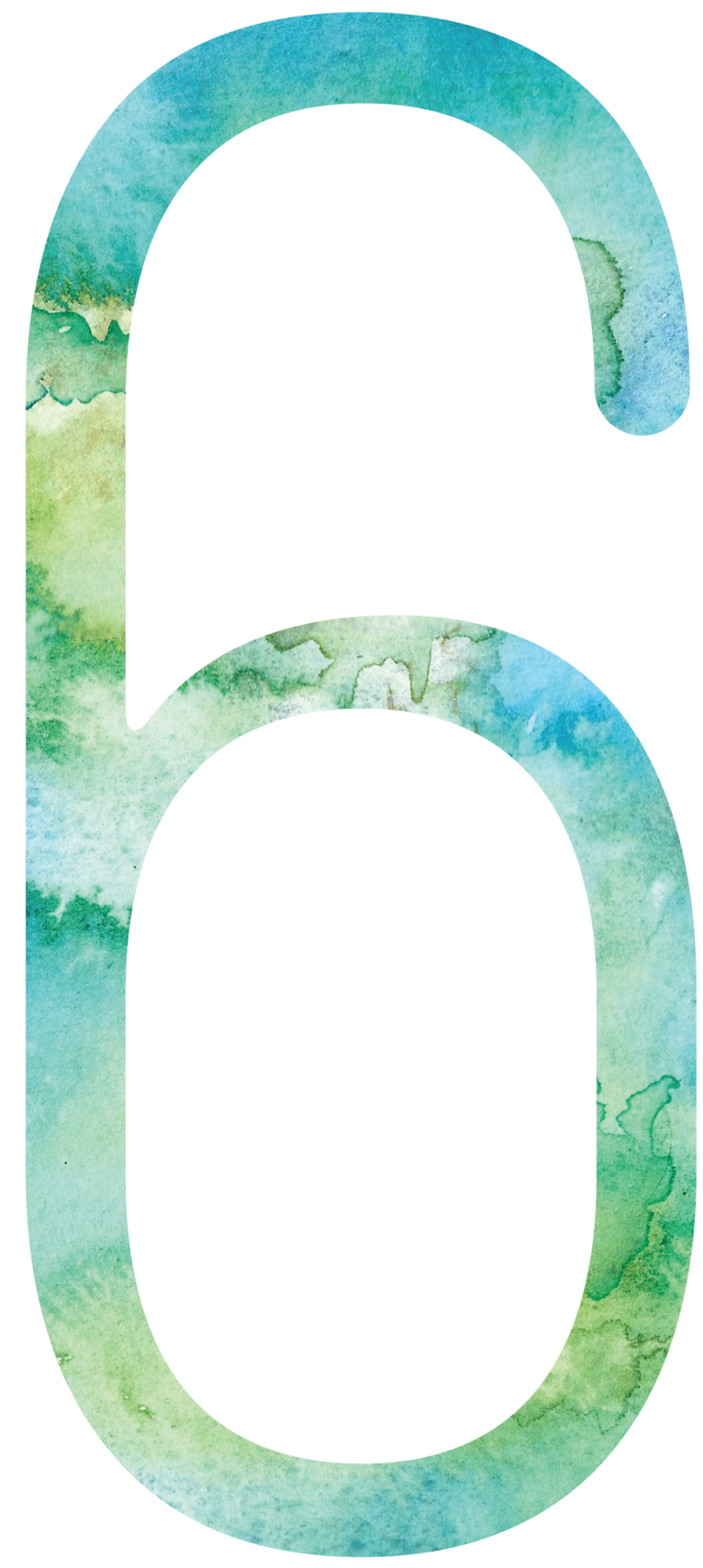


Valorization 

The research presented in this thesis focusses on innovative technological developments in quantitative ambient mass spectrometry imaging (MSI) for the pharmaceutical industry. There is a high demand for robust and validated imaging tools in the pharmaceutical pipeline. Over the last decade, MSI has established itself as one of those imaging tools that can distinguish between drug candidate and its metabolites. Early implementation of MSI in pre-clinical drug discovery and development (pharmacology) can prevent costly investments in potential drugs that proved not effective in the clinical phases. The impact of MSI on the pharmaceutical pipeline is indispensable which explains the broad interest from major pharmaceutical companies. Janssen Pharmaceutica (Janssen R\&D in Beerse, Belgium) has expressed interest in applying MSI workflows in their laboratories alongside the existing LC-MS analyses. The collaborative research program between Janssen R\&D and the Maastricht MultiModal Molecular Imaging (M4I) Institute was established in 2015. Janssen R\&D not only provided funding but even more important supported this research program with drug candidates, tissue samples, and valuable expertise. Together with Janssen R\&D, new research ideas were initiated, developed, and applied to their samples. Not surprisingly, the research presented in this thesis has led to the application of these new techniques and products that are directly translatable to their drug development process. The close collaboration did not only lead to sharing new techniques/products but also to knowledge transfer. In 2015, MSI was hardly known and pursued within Janssen R\&D. Now five years later it has become an established technique, which is being used more and more in various parts of the drug discovery and development pipeline, and in a variety of therapeutic areas, like neuroscience, infectious diseases, oncology, etc. The research value of these techniques and products have been addressed in the individual thesis chapters. Here, we will briefly describe the commercial and economic impact of this research for Janssen $\mathrm{R} \& \mathrm{D}$ and other commercial collaborators.

\section{Spatial LC-MS analysis}

The collaboration between Janssen R\&D and M4I started with a project to bridge LCMS with MSI and resulted in the development of the HR-LESA- $\mu$ LC-IMS-MSE platform (Chapter 2). This platform was established together with our commercial partners from Advion (Triversa NanoMate) and Waters Corporation (Acquity UPLC ${ }^{\circledR}$ and Synapt G2Si). The use of the TriVersa NanoMate as a spatial sampler and an LC autosampler in one is since our research publication sold by Advion as an additional Triversa NanoMate module known as LESA ${ }^{\text {plus. }}$. This allows already existing LC-MS methods to be upgraded with a spatial sampler to add invaluable spatial information to the analysis. 


\section{Q-MSI in pharmaceutical development}

The 3D printed mold for the preparation of a mimetic tissue model is a perfect example of how a collaborative environment leads to innovative ideas. After discussing a revised mimetic tissue model (Barry et al.), Darya Hadavi (a fellow M4I colleague) suggested to use a 3D printed mold for the preparation of a gelatin block. A brainstorm session resulted in a new design for a first prototype which was immediately tested in the M4I laboratory. After the prototype was optimized to the final version, Janssen requested a few extra copies to be used in their laboratory. This is the version that was used in the research reported in Chapter 5 and depicted in Figure 7.1.

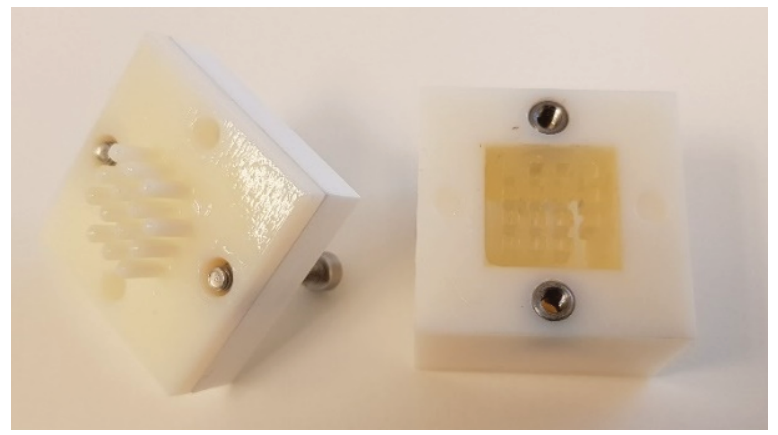

Figure 7.1 An image of the 3D printed mold that is implemented in an efficient Q-MSI workflow

\section{MRM based imaging}

At the M4l institute, the newest state-of-the-art mass spectrometers are available. This has been expressed by the introduction of M4I in the Waters Center of Innovation. Waters Corporation has been a key player on the market with innovative new MS instruments, amongst other fast high throughput triple quadrupole (QqQ) instruments. The purchase of the DESI source from Prosolia proved that their interest is becoming a major provider of ambient imaging tools. The close collaboration between Waters Corporation, M4I, and Janssen R\&D resulted in the investigation of DESI-MRM imaging and the analytical comparison of this technique with other DESI-MSI instruments/modes as presented in Chapter 4 and 5 . The setup of DESI-QqQ in the M4I laboratory is represented in Figure 7.2. The ability of performing dual polarity experiments on a QqQ instrument saves an enormous amount of time and money when two experiments can be merged into one. Shortening analysis time for known compounds in large scale drug studies with reliable instruments is in the advantage of every academic and industrial scientist. 


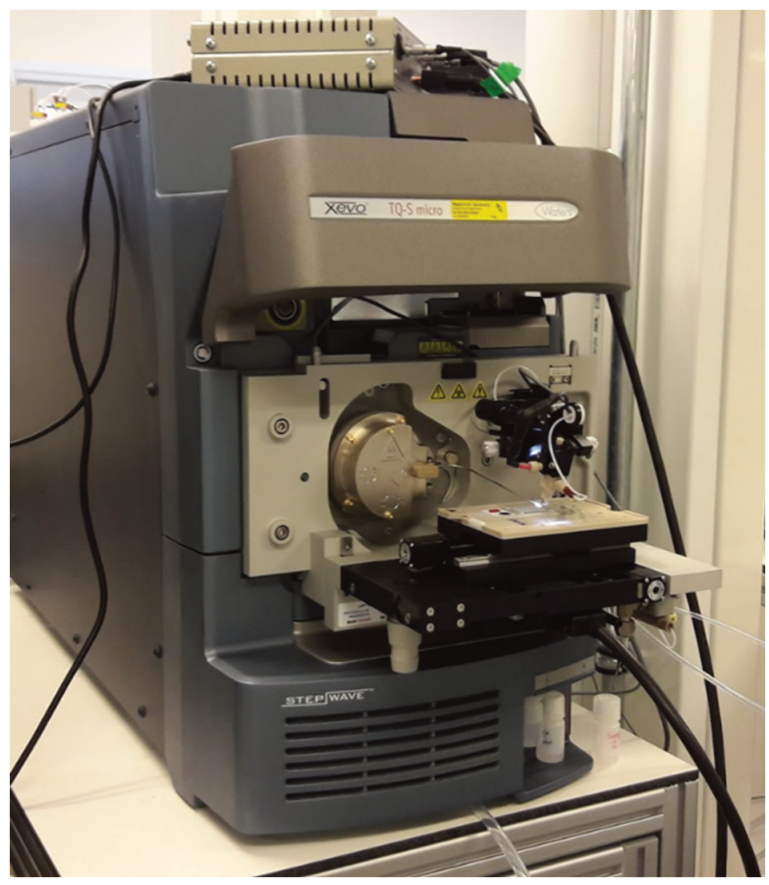

Figure 7.2 Desorption electrospray ionization source mounted onto a triple quadrupole mass spectrometer

After the multiplatform presented in Chapter 5, we believe that MRM based imaging for the pharmaceutical industry should become the standard mode of operation due to its improved analytical performance. In line with this statement, we should refer to the MALDI-QqQ instrument, Flashquant ${ }^{\mathrm{TM}}$, manufactured by Applied Biosystems in 2007 which has been taken off the market shortly after its release. One could conclude that the academic and industrial interest in MRM imaging is not significant. In 2007, the MSI field was 'only' 10 years old and very much in a developmental stage in where new imaging ion sources and MS instruments were commercialized. The investigation of applied MSI became much later. We assume that in 2007 insignificant fundamental research showed the benefit of MRM imaging leading to not enough interest in an instrument suitable for standardized routine analysis. Nowadays, MSI is much more focused on its application and is in our opinion ready for sensitive and reliable pharmaceutical MRM imaging applications that will continue to add value from fundamental and methodological research. 


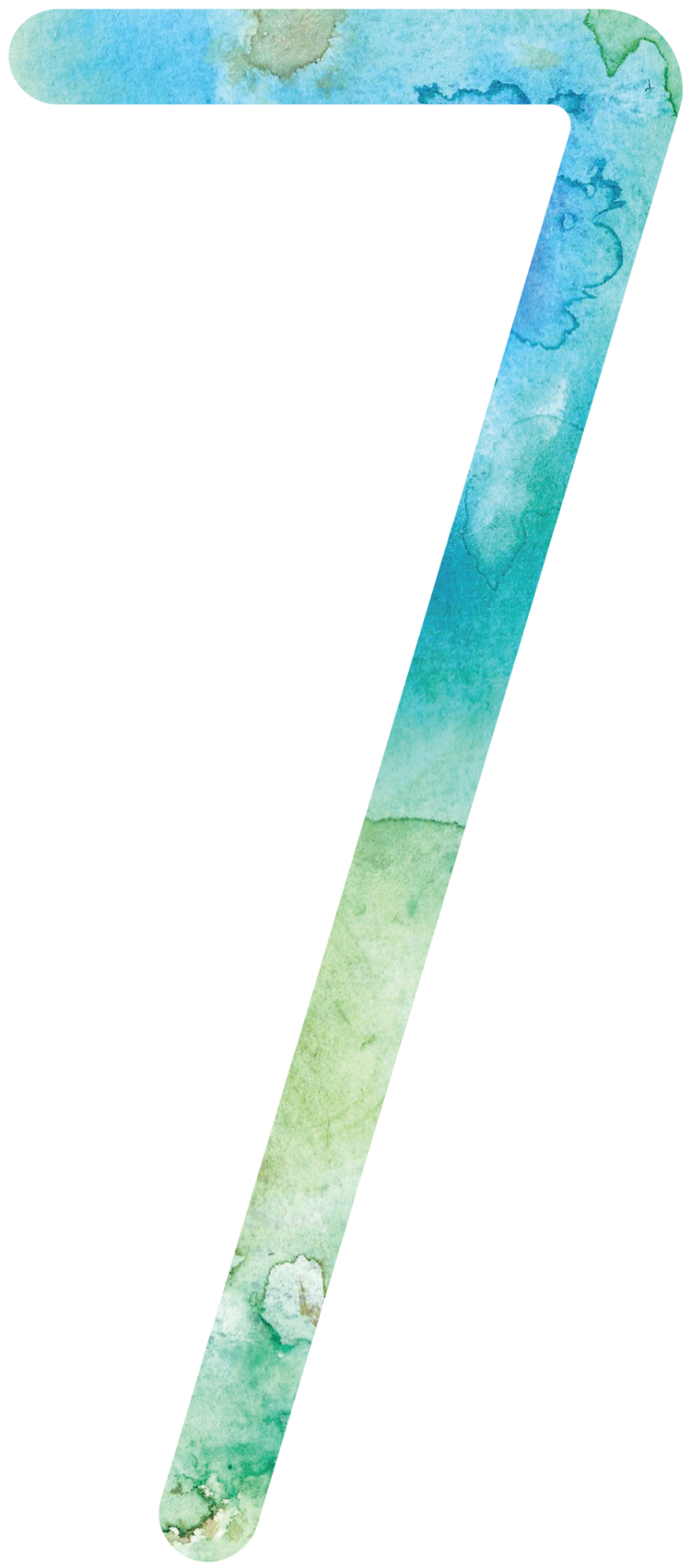


Summary and Outlook 



\subsection{General discussion}

Technological and instrumental developments in MSI have resulted in an analytical tool that provides indispensable qualitative data in the last two decades. Currently, MSI is finding its way into clinical, biomedical and pharmaceutical applications. Nevertheless, the MSI community still faces several challenges. Analysis speed needs to be increased to make MSI more suitable for high throughput routine analysis in a clinical and industrial setting. In line with the demand for routine analysis, the reproducibility and robustness of MSI workflows should be improved and standardized to meet validation criteria. Higher spatial resolution, mass resolving power and isomeric separation power could disclose a better understanding in biological processes. Identification of these new unknown imaged biomarkers is crucial as they could be markers of disease or a possible drug target. Due to limited sample cleanup abilities in MSI, only a fraction of the analytes observed in chromatographic techniques is detectable with MSI. Therefore, a more sensitive MSI approach could achieve higher compound coverage. Robust quantitative MSI (Q-MSI) workflows would reveal spatial absolute concentrations, which is on high demand in the pharmaceutical industry. All these MSI hurdles are not standalone and rely on each other.

This thesis proposes technological improvements to overcome some of these challenges and starts to address the lack of sample cleanup and ion suppression in MSI. Enhanced analyte coverage is presented by the integration of LC with an ambient spatial sampling tool, LESA. Isomeric separation has been addressed by the integration of both LC and IMS. To improve selectivity and, as a result, sensitivity in MSI, MRM based ambient imaging is presented using DESI on a QqQ instrument. In addition, this novel targeted imaging approach is very applicable in a high throughput setting ( $<30 \mathrm{~min} / \mathrm{image}$; due to the increased total detection time per precursor ion) and provides identification of known analytes. DESI-MRM imaging was subsequently further investigated for quantitative analysis. A mimetic tissue model is optimized by using a 3D-printed mold and applied in an MSI comparison which resulted in an MRM based Q-MSI workflow for pharmaceuticals.

\subsubsection{Analyte coverage}

Chapter 2 presented a novel fully automated platform that combines high resolution LESA with LC, IMS, and data independent acquisition $\left(M^{5}\right)$ to identify detected species. The occurrence of ion suppression has been a real burden for the MSI community as the majority of the MSI tools only detects the 'tip of the iceberg'. Our HR-LESA- $\mathrm{LLC}$ IMS-MSE platform removes interfering compounds from the sample prior to ionization. 
This led to an enormous increase in detected compounds directly extracted from the tissue sample. In two different tissue brain samples, a substantial number of neuropeptides, 67 and 14, have been detected and identified, while the same sampling approach without LC separation only detected 5 and 4 of those neuropeptides. This work proved that our novel platform would be an ideal tool to investigate complex biological questions that require significantly higher analyte coverage. The proposed strategy uses fast MSI analysis that can guide towards the location of interest for our HR-LESA-HLC-IMS-MSE platform.

Derivatization of analytes is a strategy to improve the ionization efficiency of poorly ionizing compounds. Chapter 3 presents the Girard's reagent T (GT) derivatization of prostanoids to overcome poor ionization in negative ionization mode. GT provides a positive charge to the carbonyl group of the prostanoid. Demonstration of endogenous prostanoid analysis proved that the efficiency of derivatization is a way to 'show more of the iceberg' and improve analyte coverage of low-abundant molecules.

\subsubsection{Isobaric and isomeric separation}

The platform presented in Chapter 2 not only combines spatial sampling with chromatography but also uses traveling wave IMS (TWIMS) to separate isobars and removes interfering background ions from the MS spectra. The isobars in Chapter 2 are from two different compounds that, due to different charge states, are overlapping in the mass spectrum and co-elute on the LC column. TWIMS is able to separate them due to different size-to-charge ratios of the isobars. Although not as significant as with LC, the removal of interfering background ions from the MS spectrum also slightly increases the analyte coverage of the platform. TWIMS and LC both have proven to separate both isobars and isomers. In theory, the HR-LESA- $\mu$ LC-IMS-MSE platform could be applied in situations where isomeric separation is required. Our platform is a multidimensional platform that can sample tissues in the xyz-direction and integrates this with four more dimensions: chromatographic retention time, ion mobility arrival time, $m / z$ ratio, compound identification by MSE.

Isomeric separation by high resolution IMS is demonstrated in Chapter 3. A comparison between CIMS, TIMS and FAIMS showed that all high resolution IMS allows for isomeric separation of GT derivatized prostanoids. The comparison revealed advantages and limitations of linear and nonlinear IMS. Due the reduced pressure conditions, linear IMS provided the best sensitivity. Although all high resolution IMS allowed isomeric separation, not all isomers in our standard mixture were separated by all three IMS techniques. Only nonlinear FAIMS was able to separate all isomeric standards. That 
there is a need for high resolution IMS combined with MSI, is shown by the increasing number of publications that integrate both techniques ${ }^{121,122,246,352 .}$

This thesis presents several technological developments that can improve analyte coverage and isomeric separation necessary for the MSI field by the use of LC (minimize ion suppression) and IMS (removal of background ions). However, it should be mentioned that the integration of $\mathrm{LC}$ in an imaging experiment has practical limitations. The time necessary to analyze one liquid extraction is in the range of 10-30 minutes. This makes analysis of tissue surfaces a very time-consuming process. Since high throughput MSI analysis requires the experimental time for mapping tissue samples to be $<30 \mathrm{~min}$, research focusses on the integration of IMS instead of LC to improve isomeric separation power to MSI. There are several MS instruments manufactured with integrated IMS and their use with MSI ionization sources is easily established. Despite the practical limitation of LC and MSI integration, chromatographic separation prior to ionization remains the way to improve analyte coverage and reduce ion suppression.

\subsubsection{Selectivity and sensitivity}

A third option to improve separation and analyte coverage in MSI is to increase selectivity of the MS analysis. Chapter 4 presents a targeted imaging approach in which DESI is coupled to a QqQ instrument. The QqQ instrument operated in several MS/MS modes as introduced in paragraph 1.2.4. MRM imaging is compared to scanning modes and resulted in increased ion detection for selected precursor ions for lipids in rat brain tissue. Interfering background ions are eliminated from the MS analysis due to added specificity and selectivity for the targeted precursor-fragment ion transitions. Two drug candidates were analyzed in dog liver tissue with multiple MRM transitions from the same precursor and confirmed the spatial distribution of both compounds. A QqQ instrument is very suitable for dual polarity experiment because of rapidly switching ion optics. Dual polarity imaging experiments were executed detecting drug candidates/metabolites in positive ionization mode and bile acids in negative ionization mode. In addition, the QqQ instrument is a compact instrument and (relatively) not expensive to purchase which makes it very suitable in pharmaceutical industry.

The potential of MRM imaging is further investigated in Chapter 5 where the performance of MRM is compared to Q-TOF instruments in MS scan, TWIMS and MSE operational modes. This multiplatform comparison investigates selectivity of MRM, IMS and $M S^{E}$ by DESI imaging of spiked tissue homogenate calibration lines in triplicate. Limits of detection extracted from these calibration lines prove that MRM is significantly more effective in removing background ions than TWIMS and, as a result, more 
sensitive. As mentioned in this discussion, technological improvements could play are role to overcome multiple MSI hurdles. We believe that is the case for MRM imaging. The sensitivity gain is only one important aspect but could have a significantly positive effect on analysis speed, spatial resolution, reproducibility, and quantification. For instance, an imaging technique with higher sensitivity, like MRM imaging, can be used to analyze the same tissue section faster or at a smaller pixel size. In the latter case, the higher sensitivity is necessary because of the decreased number of molecules/ions per pixel. Higher sensitivity will allow detection of lower abundant species with better precision due to higher signal-to-noise ratios. This translates to more accurate quantification of targeted analytes in tissues.

\subsubsection{Quantification}

A large number of research and reviews have been published about quantification in MSI as this is one of the major challenges. Due to tissue variability, the application of calibration standards is an ongoing topic of discussion. Calibration standards are spotted on top of tissues and below tissue sections. In an ideal situation, these standards would be mixed with the tissue to best mimic the tissue environment and correct for extraction and ionization efficiencies. Therefore, we chose for the application of a mimetic tissue model. Chapter 5 demonstrates MRM imaging in an adapted Q-MSI workflow. First, the practical application of a mimetic tissue model is improved by using a 3D printed mold for the preparation of a gelatin block. This gelatin block is designed with 15 squared holes for the application of the tissue homogenate samples. With total dimension of $2 \times 2 \mathrm{~cm}$, the gelatin block is easily positioned beside the tissue sample on the glass slide. The depth of the holes is $8 \mathrm{~mm}$ which makes the block suitable for large scale Q-MSI analysis. Our adapted mimetic tissue model allows not only for an 'in-tissue' Q-MSI approach with the sample tissue and mimetic tissue model on the same glass slide but is also applicable to large drug studies.

In our multiplatform comparison, the analytical performance of all instruments is determined for two drug candidates. Sensitivity, linearity, precision, and accuracy are calculated for all instruments in all modes. The combination of these validation parameters proved in significant favor of MRM imaging for two drug candidates. Now that the MSI community works towards standardization in its workflows, we believe that MRM imaging can lead to an accurate and reproducible quantitative analytical imaging tool. LC-MRM is currently the preferred tool for routine quantitative analysis in the pharmaceutical industry. Our quantitative MRM imaging work is presented as a first step towards a standardized industrial workflow. Bioanalytical methods in the pharmaceutical industry perform under standardization guidelines from authorities (i.e. 
FDA or EMA). These guidelines are constructed for analytical methods that involve chromatography, such as LC-MRM, and MRM based Q-MSI strategies still have some steps to make. However, our work proved to significantly contribute to a better performance of the Q-MSI analysis.

The use of an internal standard (IS), a stable isotope-labelled analogue of the drug candidate, is the ideal strategy to correct for ion suppression and variation throughout the analysis. However, the application of the IS with a mimetic tissue model has practical challenges, such as homogenously spraying the IS over an imperfectly cryosectioned tissue sample. In Chapter 5, we offer an alternative for spraying an internal standard on top or below the tissue sample. In drug discovery and development, an IS of the drug candidate is often not available. We present a workflow in which an endogenous cell membrane lipid is used for correction. Public software (Qupath) is used to perform a cell count analysis on the H\&E image of the tissue sample and color these cells. This H\&E image is overlaid with the MS images and overlapping pixels were extracted and quantified with the spiked tissue homogenates from the mimetic tissue model. Where legitimate, the absolute concentrations were compared to absolute concentrations obtained by LC-MRM. Our approach proved to be comparable to LC-MRM with concentration differences by a factor of $<3.5$. The measured concentration levels by QMSI are in the range of $\mu \mathrm{g} / \mathrm{g}$ tissue at $50 \mu \mathrm{m}$ pixel size while LC-MRM often reaches limits of detection in the range of $\mathrm{ng} / \mathrm{g}$ or even $\mathrm{pg} / \mathrm{g}$ tissue. Laser capture microdissection (LCM) combined with LC-MRM is a strategy to achieve regional absolute quantification in tissue samples. This approach was investigated for quantitative drug imaging, however, we came across a few weaknesses. Dehydration of the cryosectioned tissue section is needed to prevent disruption in the LCM process. Dehydration is often performed by washing the tissue section in an organic solvent and, as a result, would delocalize and/or remove the drug compound. The second observation was that LCM-LC-MRM is a time-consuming approach for one tissue section. From a practical point of view, we believe that the feasibility of LCM-LC-MRM is low for large scale drug imaging studies. However, as suggested for HR-LESA- $\mu L C$ IMS-MSE, an MSI guided application of quantitative LCM-LC-MRM can give insight in the pharmacological process.

In summary, this thesis highlights the importance of enhanced separation in MSI using ambient tissue sampling tools and offers new technologies to address the main challenges associated with Q-MSI: i) Integration of spatial sampling with LC and IMS, ii) High resolution IMS, and iii) MRM imaging. These new technologies lead to increased number of analytes detected, better isomeric separation power, higher selectivity and 
sensitivity, and ultimately results in a robust and reproducible MRM based quantitative imaging workflow for pharmaceuticals. 


\subsection{Summary}

In the pharmaceutical industry the development and application of good separation methods is important to study the effect of a potential drug. These techniques allow us to expand our knowledge of the disease and the systemic reaction of a drug and corresponding metabolites. The effect of a potential drug is studied by executing pharmacodynamics and pharmacokinetic studies (pharmacology). Pharmacology determines what a drug in development does to the human body (pharmacodynamics) and what the human body does to the drug (pharmacokinetics). After administration, the aim of the drug is to metabolize and being transported to the site of disease. The desired effect is that the drug binds to the biological receptors following by the inhibition of the processes that cause the disease. The binding to these biological receptors has to occur for a certain amount of time at a high enough drug concentration. To monitor this process, analytical techniques have to meet certain criteria: i) being able to measure absolute concentrations, ii) the location of the potential drug has to be measurable, and iii) both the potential drug and related metabolites have to be separately measured in those pharmacological studies. This is currently done by whole body autoradiography (WBA), liquid chromatography mass spectrometry (LC$\mathrm{MS})$, and mass spectrometry imaging (MSI).

In the past two decades, MSI has developed to a technique with enormous additional value to the pharmaceutical industry because it is able to separate drug from metabolites and besides this able to measure both their location in tissue samples. MSI meets two of the three technical requirements for the pharmaceutical industry. The measurement of absolute concentrations is a challenge for the MSI community. A huge burden is the phenomenon ion suppression. This is caused by the presence (besides the drug and metabolites) of other surrounding sample molecules that interfere in the ionization process of the drug and metabolites, which leads to a less optimal detection of the drug and metabolites. This thesis presents a few novel technological developments in MSI that can contribute to improved quantitative MSI analyses of drugs and metabolites.

Chapter 2 presents a platform that combines LC-MS with MSI, called high resolution liquid extraction surface analysis micro liquid chromatography ion mobility separation data independent acquisition (HR-LESA- $\mu L C-I M S-M S$ E). This platform shows the strength of complementary separation. First, HR-LESA sampling is performed with a spatial resolution of $400 \mu \mathrm{m}$. Then, after a short online washing step to remove salts, a chromatographic separation $(\mu \mathrm{LC})$ is executed followed by an ion mobility spectrometry (IMS) analysis and data independent acquisition (MSE). Two different neuropeptide applications are exhibited: the analysis of rat pituitary gland and the analysis of 
knockout and control mice brain for an Alzheimer's disease model. The analytical platform is not only highly reproducible but more importantly also allows isobaric separation by $\mu \mathrm{LC}$ and IMS and a significant increased number of detected neuropeptides due to the $\mu \mathrm{LC}$ sample separation prior to ionization.

The use of high resolution IMS is further investigated in Chapter 3. Linear cyclic IMS (CIMS), linear trapped IMS (TIMS), and nonlinear field asymmetric waveform IMS (FAIMS) are compared for the isomeric separation of ten prostanoids, including the biologically relevant prostaglandin $\mathrm{D}_{2}\left(\mathrm{PGD}_{2}\right)$ and prostaglandin $\mathrm{E}_{2}\left(\mathrm{PGE}_{2}\right)$. These prostanoids are derivatized with Girard's reagent $T$ to improve their ionization efficiency. A significant difference is observed between linear and nonlinear IMS in the IMS comparison. Linear IMS shows the highest intensity and nonlinear IMS the best resolution due to its orthogonality to MS. Another huge difference is that CIMS and TIMS occur in vacuum while FAIMS is executed at atmospheric pressure. This causes lower ion transmission in FAIMS due to collisions with surrounding molecules. That a high total transmission of ions is crucial for a technique that separates after ionization, is shown by the analysis of endogenous prostanoids in mini pig intestine tissue. The limited ion transmission in FAIMS causes, in combination with ion suppression, for a low number of detected ions. Linear IMS shows a higher intensity, however, no separation of the biologically relevant $\mathrm{PGD}_{2}$ and $\mathrm{PGE}_{2}$ is observed. Our research shows the potential of high resolution IMS as a tool for isomeric separation of prostanoids.

Chapter 4 introduces a targeted imaging strategy, named desorption electrospray ionization (DESI) multiple reaction monitoring (MRM) imaging. This work reports a lipid application and a drug and metabolite application which shows the benefit of MRM imaging. MRM imaging has the following advantages: i) increased specificity and selectivity due to the different MRM transitions of isobaric precursor ions, ii) by multiple MRM transitions from the same precursor ion, local distribution from the analyte can be confirmed, and iii) fast optical switching possibility in a triple quadrupole mass spectrometer (QqQ) allows positive and negative ions to be detected in one experiment. The measurement in tandem mass spectrometry (MS/MS) scan imaging mode is compared with by MRM imaging of the same precursor ions. Because the first and third quadrupole operate in selection mode rather than scanning mode in the same time period, sensitivity has significantly increased for these precursor ions.

The increased sensitivity of DESI-MRM is further investigated as technique for quantitative MSI (Q-MSI) in Chapter 5. Quantification is executed by use of an optimized mimetic tissue model. A gelatin block is prepared from a new designed 3D printed mold with 15 pilars to which calibration lines from tissue homogenates are added. MSI 
experiments are performed with different mass spectrometers in different modes: i) Xevo G2 Q-TOF in MS scan mode, ii) Synapt G2-Si Q-TOF in MS scan, IMS-MS scan, and IMS-MSE scan modes, and iii) Xevo TQS micro QqQ in MS scan and MRM modes. The limit of detection, linearity, precision, and accuracy are determined for each of the MS modes and compared with each other. Overall, the analytical performance of MRM imaging proved the best and, therefore, DESI-MRM is used for the quantitative analyses of four dog livers. The comparison of absolute concentrations from Q-MSI with LCMRM is debatable because regional differences get lost in the homogenization of tissue samples in the LC-MRM analysis. For this reason we only compare regional liver parenchyma (due to its substantial contribution to liver homogenates) Q-MSI concentrations with the LC-MRM data: the absolute concentrations differ by a factor of $<3.5$.

The technological developments presented in this thesis contribute to an increased number of detected analytes, better isobaric and isomeric separation, higher selectivity and sensitivity, and ultimately in improved quantitative performance of ambient MSI. 



\subsection{Samenvatting}

In de farmaceutische industrie is het van belang dat goede scheidingsmethode ontwikkeld en toegepast worden om het effect van een potentieel medicijn in kaart te brengen. Deze technieken staan ons toe om onze kennis van het ziektebeeld en de systemische reactie van een medicijn en bijbehorende metabolieten te vergroten. Het effect van een potentieel medicijn wordt gevolgd door het uitvoeren van farmacodynamische en farmacokinetische studies (farmacologie). In farmacologie wordt onderzocht wat een medicijn in ontwikkeling doet met het menselijk lichaam (farmacodynamiek) en wat het menselijk lichaam doet met dit potentiele medicijn (farmacokinetiek). Het doel van het medicijn is om na toediening gemetaboliseerd te worden en verplaatst naar de plaats van de ziekte. Hier is het gewenste effect dat het medicijn dan bindt aan biologische receptoren die vervolgens de processen die betrokken zijn bij de ziekte tegen werken. De binding aan de biologische receptoren moet voor een bepaalde tijd met een medicijnconcentratie die hoog genoeg is. Om dit goed te bestuderen moet de analytische techniek(en) aan een aantal criteria voldoen: i) de absolute concentratie moet gemeten kunnen worden, ii) de locatie van het potentiele medicijn moet gemeten kunnen worden, en iii) zowel het potentiele medicijn als gerelateerde metabolieten moeten in deze farmacologische studies gescheiden kunnen worden. Dit wordt momenteel gemeten door autoradiografie van het hele lichaam (WBA), vloeistofchromatografie-massaspectrometrie (LC-MS) en massaspectrometrie imaging (MSI).

MSI heeft zich in de afgelopen twee decennia ontwikkeld tot een techniek met een enorme toegevoegde waarde voor de farmaceutische industrie omdat het in staat is om medicijnen van metabolieten te scheiden en daarnaast in staat is om de locatie van deze moleculen in weefsels te meten. Hiermee is MSI in staat om aan twee van de drie vereisten voor de farmaceutische industrie te voldoen. Het meten van absolute concentraties met MSI is een uitdaging voor de MSI-gemeenschap. De grootste drempel hierin is het fenomeen ion suppressie. Dit wordt veroorzaakt doordat (naast het aanwezige medicijn en metabolieten) het monster ook bestaat uit andere moleculen die het ionisatieproces van het medicijn en zijn metabolieten kunnen verstoren waardoor deze minder goed ioniseren en daardoor deze minder goed te detecteren zijn. Om het effect van ion suppressie te minimaliseren presenteert dit proefschrift een aantal technologische ontwikkelingen in MSI die kunnen bijdragen aan een verbeterde kwantitatieve MSI-analyse van medicijnen en metabolieten.

Hoofdstuk 2 presenteert een platform dat LC-MS combineert met MSI, genaamd hoge resolutie vloeistof extractie oppervlakte analyse micro vloeistofchromatografie ionmobiliteitsspectrometrie data onafhankelijke acquisitie (HR-LESA- $\mu$ LC-IMS-MSE). Dit 
platform laat de kracht van complementaire scheiding zien. Allereerst wordt aangetoond dat de monsterneming met HR-LESA uitgevoerd wordt met een lokale resolutie van $400 \mu \mathrm{m}$. Vervolgens wordt, na een korte online wasstap om zouten te verwijderen, een chromatografische scheiding $(\mu \mathrm{LC})$ uitgevoerd welke opgevolgd wordt met een ionmobitiliteitsspectrometrie (IMS) analyse en een data onafhankelijke acquisitie (MSE). Twee verschillende neuropeptide applicaties worden getoond: de analyse van de hypofyse van ratten en de analyse van zieke en controle muishersenen uit een model waarin de ziekte van Alzheimer wordt onderzocht. Het analytische platform is niet alleen hoge reproduceerbaarheid maar vooral ook isobare scheiding door middel van $\mathrm{LLC}$ en IMS en een significante toename van het aantal gedetecteerde neuropeptiden door monsterscheiding voor de ionisatiestap door $\mu \mathrm{LC}$.

Het gebruik van hoge resolutie IMS wordt verder bestudeerd in Hoofdstuk 3. Hierin worden lineaire cyclische IMS (CIMS), lineaire gevangen IMS (TIMS) en niet-lineaire veld asymmetrische golfvorm IMS (FAIMS) met elkaar vergeleken voor de isomere scheiding van een tiental prostanoiden, waaronder biologische relevante prostaglandine $D_{2}$ $\left(P D_{2}\right)$ en prostaglandine $E_{2}\left(P_{G} E_{2}\right)$. Deze prostanoiden worden eerst gederivatiseerd met Girard's reagens T om de ionisatie efficiëntie te vergroten. In de IMS-vergelijking wordt een significant verschil tussen lineaire en niet-lineaire IMS waargenomen. Lineaire IMS laat de hoogste intensiteit zien en niet-lineaire IMS de beste resolutie door zijn orthogonaliteit ten opzichte van MS. Een ander groot verschil is dat cIMS en TIMS in vacuüm plaatsvindt terwijl FAIMS bij atmosferisch druk uitgevoerd wordt. Dit zorgt ervoor dat de ion transmissie lager is in FAIMS door botsing met omgevingsmoleculen. Dat de totale transmissie van ionen cruciaal is in een techniek die na de ionisatiestap scheidt, blijkt uit de analyse van endogene prostanoiden in minivarken darmweefsel. De beperkte ion transmissie in FAIMS zorgt er, in combinatie met ion suppressie, voor dat een minimaal aantal ionen gedetecteerd wordt. Lineaire IMS laat een hogere intensiteit zijn maar er is daarentegen geen scheiding geobserveerd voor de biologische relevante isomeren $\mathrm{PGD}_{2}$ en $\mathrm{PGE}_{2}$. Ons onderzoek laat zien dat hoge resolutie IMS een techniek kan zijn voor isomere scheiding van prostanoiden.

Hoofdstuk 4 introduceert een doelgerichte imaging analyse strategie, genaamd desorptie electrospray isonisatie (DESI) meerdere reactie monitoring (MRM) imaging. Dit werk rapporteert een lipiden en medicijn en zijn metaboliet applicatie waarin het voordeel van MRM imaging wordt gepresenteerd. MRM imaging heeft de volgende voordelen: i) vergrootte specificiteit en selectiviteit door de verschillende MRM overgangen van isobare moederionen, ii) met meerdere MRM overgangen van hetzelfde moederion kan de lokale distributie van dit analiet bevestigd worden, en iii) door de snelle optische omschakkelingsmogelijkheid van een drievoudige quadrupool 
massaspectrometer (QqQ) kunnen positieve en negatieve ionen in een experiment gedetecteerd worden. Het meten in een tandem massaspectrometrie (MS/MS) scan imaging modes wordt vergeleken met MRM imaging voor dezelfde moederionen. Doordat de eerste en derde quadrupool nu in selectie modes opereert in plaats van scanning modes in hetzelfde tijdsbereik, is de gevoeligheid significant toegenomen voor deze moederionen.

Deze gevoeligheidstoename van DESI-MRM wordt verder onderzocht als techniek voor kwantitatieve MSI (Q-MSI) in Hoofdstuk 5. Kwantificatie wordt uitgevoerd door middel van een geoptimaliseerd nabootsend weefselmodel. Hierin wordt een gelatineblok gemaakt uit een nieuw ontworpen 3D geprinte gietvorm met 15 pilaren waarin vervolgens kalibratielijnen van weefselhomogenaten wordt toegevoegd. MSIexperimenten worden uitgevoerd met verschillende massaspectrometers in verschillende modes: i) Xevo G2 Q-TOF in MS scan mode, ii) Synapt G2-Si Q-TOF in MS scan, IMS-MS scan en IMS-MSE scan modes, en iii) Xevo TQS micro QqQ in MS scan en MRM modes. Het detectielimiet, lineairiteit, precisie en accuraatheid wordt bepaald voor elk van de MS modes en vergeleken met elkaar. Over het algemeen is de analytische prestatie van MRM imaging het best en daarom is er voor de kwantitatieve analyse van vier hondenlevers gekozen voor DESI-MRM. De vergelijking van absolute concentraties uit Q-MSI met kwantitatieve LC-MRM experimenten is discutabel aangezien de LC-MRM analyse altijd op een gehomogeniseerde versie van het weefsel is waarin regionale verschillen verloren gaan. Om deze reden vergelijken we alleen regionale lever parenchym (door de substantiële bijdrage van lever parenchym aan de lever homogenaten) Q-MSI concentraties met de LC-MRM data: de absolute concentraties wijken een factor $<3,5$ af.

De technologische ontwikkelingen die dit proefschrift presenteert dragen bij aan een toename in het aantal te detecteren analieten, betere isobare en isomere scheiding, hogere selectiviteit en gevoeligheid en uiteindelijk in een betere kwantitatieve presentatie van MSI in omgevingsomstandigheden. 


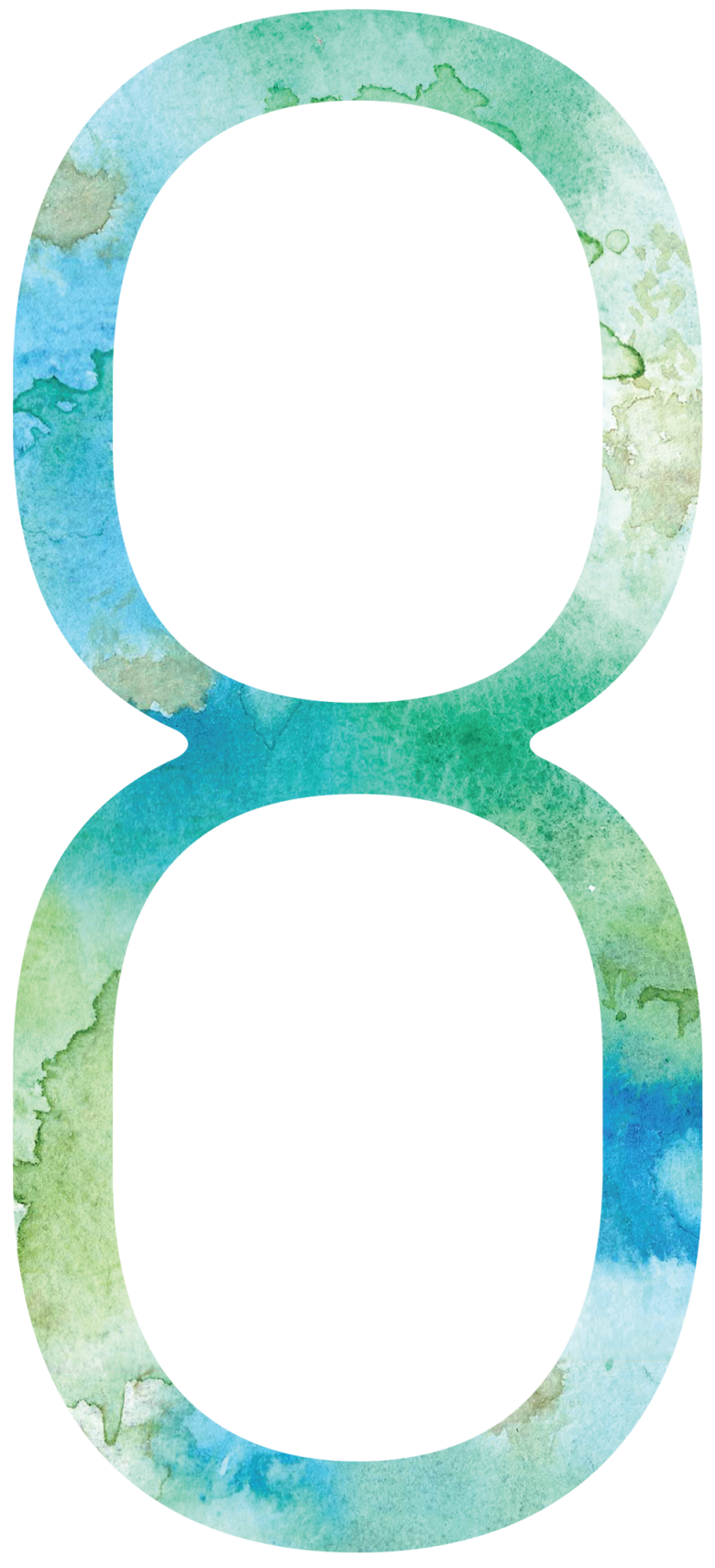


Appendices 



\subsection{References}

1. F. Adams \& M. Adriaens, The metamorphosis of analytical chemistry, Analytical and Bioanalytical Chemistry, 2020, 412, 3525-3537.

2. J. M. Green, A Practical Guide to Analytical Method Validation, Analytical Chemistry News \& Features, 1996, A, 5.

3. W. M. Niessen \& D. Falck, Introduction to Mass Spectrometry, a Tutorial, Wiley, 2015.

4. E. de Hoffmann \& V. Stroobant, Mass Spectrometry: Principles and Applications, Wiley, 2013.

5. G. L. Glish \& R. W. Vachet, The basics of mass spectrometry in the twenty-first century, Nature Reviews Drug Discovery, 2003, 2, 140-150.

6. M. Karas \& F. Hillenkamp, Laser desorption ionization of proteins with molecular masses exceeding 10,000 daltons, Analytical Chemistry, 1988, 60, 2299-2301.

7. R. Knochenmuss, Ion formation mechanisms in UV-MALDI, The Analyst, 2006, 131, 966-986.

8. J. B. Fenn, M. Mann, C. K. Meng, S. F. Wong \& C. M. Whitehouse, Electrospray ionization for mass spectrometry of large biomolecules, Science (New York, N.Y.), 1989, 246, 64.

9. C. S. Ho, C. W. K. Lam, M. H. M. Chan, R. C. K. Cheung, L. K. Law, L. C. W. Lit, K. F. Ng, M. W. M. Suen \& H. L. Tai, Electrospray ionisation mass spectrometry: principles and clinical applications, Clinical Biochemist Reviews, 2003, 24, 3-12.

10. F. A. Kero, R. A. Yost \& R. E. Pedder, Quadrupole mass analyzers: theoretical and practical considerations, 2005.

11. J. W. Hager, A new linear ion trap mass spectrometer, Rapid Communications in Mass Spectrometry, 2002, 16, 512-526.

12. B. A. Mamyrin, V. I. Karataev, D. V. Shmikk \& V. A. Zagulin, Mass reflection: a new nonmagnetic time-of-flight high resolution mass- spectrometer, Journal of Experimental and Theoretical Physics, $1973,64,82-89$.

13. A. Makarov, Electrostatic Axially Harmonic Orbital Trapping: A High-Performance Technique of Mass Analysis, Analytical Chemistry, 2000, 72, 1156-1162.

14. M. B. Comisarow \& A. G. Marshall, Fourier transform ion cyclotron resonance spectroscopy, Chemical Physics Letters, 1974, 25, 282-283.

15. R. S. Brown \& J. J. Lennon, Mass Resolution Improvement by Incorporation of Pulsed lon Extraction in a Matrix-Assisted Laser Desorption/lonization Linear Time-of-Flight Mass Spectrometer, Analytical Chemistry, 1995, 67, 1998-2003.

16. Q. Hu, R. J. Noll, H. Li, A. Makarov, M. Hardman \& R. Graham Cooks, The Orbitrap: a new mass spectrometer, Journal of Mass Spectrometry, 2005, 40, 430-443.

17. I. J. Amster, Fourier Transform Mass Spectrometry, Journal of Mass Spectrometry, 1996, 31, 13251337.

18. M. Scigelova, M. Hornshaw, A. Giannakopulos \& A. Makarov, Fourier transform mass spectrometry, Molecular \& Cellular Proteomics, 2011, 10, 1-19.

19. C. Dass, Tandem Mass Spectrometry, 2006.

20. J. V. Johnson, R. A. Yost, P. E. Kelley \& D. C. Bradford, Tandem-in-space and tandem-in-time mass spectrometry: triple quadrupoles and quadrupole ion traps, Analytical Chemistry, 1990, 62, 21622172.

21. J. Mitchell Wells \& S. A. McLuckey, in Methods in enzymology, Academic Press, 2005, vol. 402, pp. 148-185.

22. L. Sleno \& D. A. Volmer, Ion activation methods for tandem mass spectrometry, Journal of Mass Spectrometry, 2004, 39, 1091-1112.

23. E. de Hoffmann, Tandem mass spectrometry: A primer, Journal of Mass Spectrometry, 1996, 31, 129-137.

24. K. M. Kermit, K. B. Robert, N. E. Marcos, G. J. Langley, L. Liang \& N. Yasuhide, Definitions of terms relating to mass spectrometry (IUPAC Recommendations 2013), Pure and Applied Chemistry, 2013, 85, 1515-1609.

25. B. Brügger, Lipidomics: Analysis of the Lipid Composition of Cells and Subcellular Organelles by Electrospray lonization Mass Spectrometry, Annual Review of Biochemistry, 2014, 83, 79-98.

26. I. V. Chernushevich, A. V. Loboda \& B. A. Thomson, An introduction to quadrupole-time-of-flight mass spectrometry, Journal of Mass Spectrometry, 2001, 36, 849-865.

27. J. H. J. Dawson \& M. Guilhaus, Orthogonal-acceleration time-of-flight mass spectrometer, Rapid Communications in Mass Spectrometry, 1989, 3, 155-159.

28. T. Kind \& O. Fiehn, Advances in structure elucidation of small molecules using mass spectrometry, Bioanal Rev, 2010, 2, 23-60. 
29. J. L. Little, C. D. Cleven \& S. D. Brown, Identification of "Known Unknowns" Utilizing Accurate Mass Data and Chemical Abstracts Service Databases, Journal of the American Society for Mass Spectrometry, 2011, 22, 348-359.

30. K. Lorenzen \& E. v. Duijn, Native Mass Spectrometry as a Tool in Structural Biology, Current Protocols in Protein Science, 2010, 62, 17.12.11-17.12.17.

31. F. F. Hsu, F. M. Kuhlmann, J. Turk \& S. M. Beverley, Multiple-stage linear ion-trap with high resolution mass spectrometry towards complete structural characterization of phosphatidylethanolamines containing cyclopropane fatty acyl chain in Leishmania infantum, Journal of Mass Spectrometry, 2014, 49, 201-209.

32. F. F. Hsu, S. Lobasso, J. Turk \& A. Corcelli, Structural studies on archaeal phytanyl-ether lipids isolated from membranes of extreme halophiles by linear ion-trap multiple-stage tandem mass spectrometry with electrospray ionization, Analytica chimica acta, 2013, 771, 73-85.

33. F. F. Hsu, I. J. Lodhi, J. Turk \& C. F. Semenkovich, Structural distinction of diacyl-, alkylacyl, and alk1-enylacyl glycerophosphocholines as [M - 15](-) ions by multiple-stage linear ion-trap mass spectrometry with electrospray ionization, Journal of the American Society for Mass Spectrometry, 2014, 25, 1412-1420.

34. F. F. Hsu, S. Pacheco, J. Turk \& G. Purdy, Structural determination of glycopeptidolipids of Mycobacterium smegmatis by high-resolution multiple-stage linear ion-trap mass spectrometry with electrospray ionization, Journal of Mass Spectrometry, 2012, 47, 1269-1281.

35. F. F. Hsu \& J. Turk, Elucidation of the double-bond position of long-chain unsaturated fatty acids by multiple-stage linear ion-trap mass spectrometry with electrospray ionization, Journal of the American Society for Mass Spectrometry, 2008, 19, 1673-1680.

36. F. F. Hsu \& J. Turk, Structural characterization of unsaturated glycerophospholipids by multiplestage linear ion-trap mass spectrometry with electrospray ionization, Journal of the American Society for Mass Spectrometry, 2008, 19, 1681-1691.

37. F. F. Hsu \& J. Turk, Electrospray ionization multiple-stage linear ion-trap mass spectrometry for structural elucidation of triacylglycerols: assignment of fatty acyl groups on the glycerol backbone and location of double bonds, Journal of the American Society for Mass Spectrometry, 2010, 21, 657-669.

38. F. F. Hsu \& J. Turk, Toward total structural analysis of cardiolipins: multiple-stage linear ion-trap mass spectrometry on the $[\mathrm{M}-2 \mathrm{H}+3 \mathrm{Li}]+$ ions, Journal of the American Society for Mass Spectrometry, 2010, 21, 1863-1869.

39. F. F. Hsu, J. Turk, E. R. Rhoades, D. G. Russell, Y. Shi \& E. A. Groisman, Structural characterization of cardiolipin by tandem quadrupole and multiple-stage quadrupole ion-trap mass spectrometry with electrospray ionization, Journal of the American Society for Mass Spectrometry, 2005, 16, 491-504.

40. J. Clayden, N. Greeves \& S. G. Warren, Organic chemistry, Oxford University Press, Oxford; New York, 2012.

41. J. K. M. Sanders \& B. K. Hunter, Modern NMR spectroscopy: a guide for chemists, Oxford University Press, United States, 1988.

42. L. Zhang, C. Tang, D. Cao, Y. Zeng, B. Tan, M. Zeng, W. Fan, H. Xiao \& Y. Liang, Strategies for structure elucidation of small molecules using gas chromatography-mass spectrometric data, TrAC Trends in Analytical Chemistry, 2013, 47, 37-46.

43. O. Fiehn, Metabolomics by Gas Chromatography-Mass Spectrometry: Combined Targeted and Untargeted Profiling, Current Protocols in Molecular Biology, 2016, 114, 30.34.31-30.34.32.

44. R. S. Plumb, K. A. Johnson, P. Rainville, B. W. Smith, I. D. Wilson, J. M. Castro-Perez \& J. K. Nicholson, UPLC/MS(E); a new approach for generating molecular fragment information for biomarker structure elucidation, Rapid Communications in Mass Spectrom, 2006, 20, 1989-1994.

45. G. Groeneveld, M. N. Dunkle, M. Rinken, A. F. G. Gargano, A. de Niet, M. Pursch, E. P. C. Mes \& P. J. Schoenmakers, Characterization of complex polyether polyols using comprehensive twodimensional liquid chromatography hyphenated to high-resolution mass spectrometry, Journal of Chromatography A, 2018, 1569, 128-138.

46. I. Garcia-Perez, J. M. Posma, J. I. Serrano-Contreras, C. L. Boulangé, Q. Chan, G. Frost, J. Stamler, P. Elliott, J. C. Lindon, E. Holmes \& J. K. Nicholson, Identifying unknown metabolites using NMRbased metabolic profiling techniques, Nature Protocols, 2020, 15, 2538-2567.

47. C. M. Snyder, X. Zhou, J. A. Karty, B. R. Fonslow, M. V. Novotny \& S. C. Jacobson, Capillary electrophoresis-mass spectrometry for direct structural identification of serum N-glycans, Journal of Chromatography $A, 2017,1523,127-139$. 
F. P. Gomes \& J. R. Yates III, Recent trends of capillary electrophoresis-mass spectrometry in proteomics research, Mass spectrometry reviews, 2019, 38, 445-460.

49. R. Ramautar, R. Berger, J. van der Greef \& T. Hankemeier, Human metabolomics: strategies to understand biology, Current opinion in chemical biology, 2013, 17, 841-846.

50. C. S. Creaser, J. R. Griffiths, C. J. Bramwell, S. Noreen, C. A. Hill \& C. L. P. Thomas, Ion mobility spectrometry: a review. Part 1. Structural analysis by mobility measurement, The Analyst, 2004, 129, 984-994.

51. C. Lapthorn, F. Pullen \& B. Z. Chowdhry, lon mobility spectrometry-mass spectrometry (IMS-MS) of small molecules: separating and assigning structures to ions, Mass spectrometry reviews, 2013, 32, 43-71.

52. Y. Zhong, S. J. Hyung \& B. T. Ruotolo, lon mobility-mass spectrometry for structural proteomics, Expert review of proteomics, 2012, 9, 47-58.

53. J. R. Enders \& J. A. Mclean, Chiral and structural analysis of biomolecules using mass spectrometry and ion mobility-mass spectrometry, Chirality, 2009, 21, E253-E264.

54. F. Lanucara, S. W. Holman, C. J. Gray \& C. E. Eyers, The power of ion mobility-mass spectrometry for structural characterization and the study of conformational dynamics, Nature Chemistry, 2014, 6, 281-294.

55. A. B. Kanu, P. Dwivedi, M. Tam, L. Matz \& H. H. Hill, Ion mobility-mass spectrometry, Journal of Mass Spectrometry, 2008, 43, 1-22.

56. H. Borsdorf \& G. A. Eiceman, Ion Mobility Spectrometry: Principles and Applications, Applied Spectroscopy Reviews, 2006, 41, 323-375.

57. B. C. Bohrer, S. I. Merenbloom, S. L. Koeniger, A. E. Hilderbrand \& D. E. Clemmer, Biomolecule analysis by ion mobility spectrometry, Annual Review of Analytical Chemistry, 2008, 1, $293-327$.

58. A. A. Shvartsburg \& R. D. Smith, Fundamentals of Traveling Wave Ion Mobility Spectrometry, Analytical Chemistry, 2008, 80, 9689-9699.

59. D. B. Graves, Transport properties of ions in gases by Edward A. Mason and Earl W. McDaniel, John Wiley and Sons, New York, 1988, 560 + xvi pp, A/ChE Journal, 1989, 35, 701-701.

60. R. Cumeras, E. Figueras, C. E. Davis, J. I. Baumbach \& I. Gràcia, Review on ion mobility spectrometry. Part 1: current instrumentation, The Analyst, 2015, 140, 1376-1390.

61. K. Giles, S. D. Pringle, K. R. Worthington, D. Little, J. L. Wildgoose \& R. H. Bateman, Applications of a travelling wave-based radio-frequency-only stacked ring ion guide, Rapid Communications in Mass Spectrometry, 2004, 18, 2401-2414.

62. S. D. Pringle, K. Giles, J. L. Wildgoose, J. P. Williams, S. E. Slade, K. Thalassinos, R. H. Bateman, M. T. Bowers \& J. H. Scrivens, An investigation of the mobility separation of some peptide and protein ions using a new hybrid quadrupole/travelling wave IMS/oa-ToF instrument, International journal of mass spectrometry, 2007, 261, 1-12.

63. F. Fernandez-Lima, D. A. Kaplan, J. Suetering \& M. A. Park, Gas-phase separation using a trapped ion mobility spectrometer, International Journal of Ion Mobility Spectrometry, 2011, 14.

64. K. Michelmann, J. A. Silveira, M. E. Ridgeway \& M. A. Park, Fundamentals of trapped ion mobility spectrometry, Journal of the American Society for Mass Spectrometry, 2015, 26, 14-24.

65. R. Guevremont, High-field asymmetric waveform ion mobility spectrometry: a new tool for mass spectrometry, Journal of Chromatography A, 2004, 1058, 3-19.

66. R. Guevremont \& R. W. Purves, Atmospheric pressure ion focusing in a high-field asymmetric waveform ion mobility spectrometer, Review of Scientific Instruments, 1999, 70, 1370-1383.

67. R. W. Purves \& R. Guevremont, Electrospray Ionization High-Field Asymmetric Waveform Ion Mobility Spectrometry-Mass Spectrometry, Analytical Chemistry, 1999, 71, 2346-2357.

68. A. A. Shvartsburg, T. Bryskiewicz, R. W. Purves, K. Tang, R. Guevremont \& R. D. Smith, Field Asymmetric Waveform Ion Mobility Spectrometry Studies of Proteins: Dipole Alignment in Ion Mobility Spectrometry?, Journal of Physical Chemistry B, 2006, 110, 21966-21980.

69. H. H. Hill, W. F. Siems \& R. H. St. Louis, Ion mobility spectrometry, Analytical Chemistry, 1990, 62 , 1201A-1209A.

70. M. E. Ridgeway, C. Bleiholder, M. Mann \& M. A. Park, Trends in trapped ion mobility - Mass spectrometry instrumentation, TrAC Trends in Analytical Chemistry, 2019, 116, 324-331.

71. Y. Wang, X. Wang, L. Li, C. Chen, T. Xu, T. Wang \& J. Luo, High pressure effects in high-field asymmetric waveform ion mobility spectrometry, Rapid Communications in Mass Spectrometry, 2016, 30, 1914-1922.

72. A. Garabedian, M. A. Baird, J. Porter, K. Jeanne Dit Fouque, P. V. Shliaha, O. N. Jensen, T. D. Williams, F. Fernandez-Lima \& A. A. Shvartsburg, Linear and Differential Ion Mobility Separations of Middle-Down Proteoforms, Analytical Chemistry, 2018, 90, 2918-2925. 
73. D. A. Skoog, S. R. Crouch \& F. J. Holler, Principles of instrumental analysis, Thomson Brooks/Cole, Belmont, CA, 2007.

74. S. Fekete, I. Kohler, S. Rudaz \& D. Guillarme, Importance of instrumentation for fast liquid chromatography in pharmaceutical analysis, Journal of pharmaceutical and biomedical analysis, 2014, 87, 105-119.

75. D. Cabooter, A. Fanigliulo, G. Bellazzi, B. Allieri, A. Rottigni \& G. Desmet, Relationship between the particle size distribution of commercial fully porous and superficially porous high-performance liquid chromatography column packings and their chromatographic performance, Journal of Chromatography A, 2010, 1217, 7074-7081.

76. J. J. van Deemter, F. J. Zuiderweg \& A. Klinkenberg, Longitudinal diffusion and resistance to mass transfer as causes of nonideality in chromatography, Chemical Engineering Science, 1995, 50, 3869-3882.

77. M. E. Swartz, UPLC ${ }^{\mathrm{TM}}$ : An Introduction and Review, Journal of Liquid Chromatography \& Related Technologies, 2005, 28, $1253-1263$.

78. R. M. Caprioli, T. B. Farmer \& J. Gile, Molecular imaging of biological samples: localization of peptides and proteins using MALDI-TOF MS, Analytical Chemistry, 1997, 69, 4751-4760.

79. D. Bonnel, R. Legouffe, N. Willand, A. Baulard, G. Hamm, B. Deprez \& J. Stauber, MALDI imaging techniques dedicated to drug-distribution studies, Bioanalysis, 2011, 3, 1399-1406.

80. T. Greer, R. Sturm \& L. Li, Mass spectrometry imaging for drugs and metabolites, Journal of Proteomics, 2011, 74, 2617-2631.

81. H. Loden, M. Shariatgorji, A. Nilsson \& P. E. Andren, An introduction to MS imaging in drug discovery and development, Bioanalysis, 2015, 7, 2621-2627.

82. A. Nilsson, R. J. Goodwin, M. Shariatgorji, T. Vallianatou, P. J. Webborn \& P. E. Andren, Mass spectrometry imaging in drug development, Analytical Chemistry, 2015, 87, 1437-1455.

83. B. Prideaux \& M. Stoeckli, Mass spectrometry imaging for drug distribution studies, Journal of Proteomics, 2012, 75, 4999-5013.

84. H. Meistermann, J. L. Norris, H. R. Aerni, D. S. Cornett, A. Friedlein, A. R. Erskine, A. Augustin, M. C. De Vera Mudry, S. Ruepp, L. Suter, H. Langen, R. M. Caprioli \& A. Ducret, Biomarker discovery by imaging mass spectrometry: transthyretin is a biomarker for gentamicin-induced nephrotoxicity in rat, Molecular \& Cellular Proteomics, 2006, 5, 1876-1886.

85. K. Schwamborn, Imaging mass spectrometry in biomarker discovery and validation, Journal of Proteomics, 2012, 75, 4990-4998.

86. K. Chughtai \& R. M. A. Heeren, Mass spectrometric imaging for biomedical tissue analysis, Chemical reviews, 2010, 110, 3237-3277.

87. B. M. Prentice, R. M. Caprioli \& V. Vuiblet, Label-free molecular imaging of the kidney, Kidney international, 2017, 92, 580-598.

88. P.-M. Vaysse, R. M. A. Heeren, T. Porta \& B. Balluff, Mass spectrometry imaging for clinical research - latest developments, applications, and current limitations, The Analyst, 2017, 142, 2690-2712.

89. J. Kriegsmann, M. Kriegsmann \& R. Casadonte, MALDI TOF imaging mass spectrometry in clinical pathology: A valuable tool for cancer diagnostics (Review), International journal of oncology, 2015, 46, 893-906.

90. K. Scupakova, B. Balluff, C. Tressler, T. Adelaja, R. M. A. Heeren, K. Glunde \& G. Ertaylan, Cellular resolution in clinical MALDI mass spectrometry imaging: the latest advancements and current challenges, Clinical chemistry and laboratory medicine, 2019, 58, 914-929.

91. L. A. McDonnell \& R. M. Heeren, Imaging mass spectrometry, Mass spectrometry reviews, 2007, 26, 606-643.

92. R. M. A. Heeren, D. F. Smith, J. Stauber, B. Kükrer-Kaletas \& L. MacAleese, Imaging Mass Spectrometry: Hype or Hope?, Journal of the American Society for Mass Spectrometry, 2009, 20, 1006-1014.

93. R. M. Caprioli, Imaging Mass Spectrometry - Molecular Microscopy for Biological and Clinical Research, 2017, 115-132.

94. A. R. Buchberger, K. DeLaney, J. Johnson \& L. Li, Mass Spectrometry Imaging: A Review of Emerging Advancements and Future Insights, Analytical Chemistry, 2018, 90, 240-265.

95. A. L. Bruinen, G. L. Fisher, R. Balez, A. M. van der Sar, L. Ooi \& R. M. A. Heeren, Identification and High-Resolution Imaging of $\alpha$-Tocopherol from Human Cells to Whole Animals by TOF-SIMS Tandem Mass Spectrometry, Journal of the American Society for Mass Spectrometry, 2018, 29, 1571-1581.

96. M. L. Reyzer \& R. M. Caprioli, MALDI-MS-based imaging of small molecules and proteins in tissues, Current opinion in chemical biology, 2007, 11, 29-35. 
S. J. Pachuta \& R. G. Cooks, Mechanisms in molecular SIMS, Chemical reviews, 1987, 87, 647669.

98. H. W. Werner, The use of secondary ion mass spectrometry in surface analysis, Surface Science, 1975, 47, 301-323.

99. L. S. Eberlin, C. R. Ferreira, A. L. Dill, D. R. Ifa \& R. G. Cooks, Desorption electrospray ionization mass spectrometry for lipid characterization and biological tissue imaging, Biochim Biophys Acta, 2011, 1811, 946-960.

100. M. W. Towers, T. Karancsi, E. A. Jones, S. D. Pringle \& E. Claude, Optimised Desorption Electrospray Ionisation Mass Spectrometry Imaging (DESI-MSI) for the Analysis of Proteins/Peptides Directly from Tissue Sections on a Travelling Wave Ion Mobility Q-ToF, Journal of the American Society for Mass Spectrometry, 2018, 29, 2456-2466.

101. F. Dewez, M. Martin-Lorenzo, M. Herfs, D. Baiwir, G. Mazzucchelli, E. De Pauw, R. M. A. Heeren \& B. Balluff, Precise co-registration of mass spectrometry imaging, histology, and laser microdissection-based omics, Analytical and Bioanalytical Chemistry, 2019, 411, 5647-5653.

102. A. P. Bowman, J. F. J. Bogie, J. J. A. Hendriks, M. Haidar, M. Belov, R. M. A. Heeren \& S. R. Ellis, Evaluation of lipid coverage and high spatial resolution MALDI-imaging capabilities of oversampling combined with laser post-ionisation, Analytical and Bioanalytical Chemistry, 2020, 412, 22772289.

103. A. D. Feenstra, M. E. Dueñas \& Y. J. Lee, Five Micron High Resolution MALDI Mass Spectrometry Imaging with Simple, Interchangeable, Multi-Resolution Optical System, Journal of the American Society for Mass Spectrometry, 2017, 28, 434-442.

104. M. Kompauer, S. Heiles \& B. Spengler, Atmospheric pressure MALDI mass spectrometry imaging of tissues and cells at 1.4- $\mu$ m lateral resolution, Nature Methods, 2017, 14, 90-96.

105. J. Franck, K. Arafah, A. Barnes, M. Wisztorski, M. Salzet \& I. Fournier, Improving tissue preparation for matrix-assisted laser desorption ionization mass spectrometry imaging. Part 1: using microspotting, Analytical Chemistry, 2009, 81, 8193-8202.

106. E. R. Amstalden van Hove, D. F. Smith, L. Fornai, K. Glunde \& R. M. A. Heeren, An Alternative Paper Based Tissue Washing Method for Mass Spectrometry Imaging: Localized Washing and Fragile Tissue Analysis, Journal of the American Society for Mass Spectrometry, 2011, 22.

107. B. Heijs, E. A. Tolner, J. V. Bovée, A. M. van den Maagdenberg \& L. A. McDonnell, Brain RegionSpecific Dynamics of On-Tissue Protein Digestion Using MALDI Mass Spectrometry Imaging, Journal of Proteome Research, 2015, 14, 5348-5354.

108. B. Cillero-Pastor \& R. M. Heeren, Matrix-assisted laser desorption ionization mass spectrometry imaging for peptide and protein analyses: a critical review of on-tissue digestion, Journal of Proteome Research, 2014, 13, 325-335.

109. Q. Wu, T. J. Comi, B. Li, S. S. Rubakhin \& J. V. Sweedler, On-Tissue Derivatization via Electrospray Deposition for Matrix-Assisted Laser Desorption/Ionization Mass Spectrometry Imaging of Endogenous Fatty Acids in Rat Brain Tissues, Analytical Chemistry, 2016, 88, 5988-5995.

110. F. P. Y. Barré, B. Flinders, J. P. Garcia, I. Jansen, L. R. S. Huizing, T. Porta, L. B. Creemers, R. M. A. Heeren \& B. Cillero-Pastor, Derivatization Strategies for the Detection of Triamcinolone Acetonide in Cartilage by Using Matrix-Assisted Laser Desorption/lonization Mass Spectrometry Imaging, Analytical Chemistry, 2016, 88, 12051-12059.

111. R. Shariatgorji, A. Nilsson, N. Strittmatter, T. Vallianatou, X. Zhang, P. Svenningsson, R. J. A. Goodwin \& P. E. Andrén, Bromopyrylium Derivatization Facilitates Identification by Mass Spectrometry Imaging of Monoamine Neurotransmitters and Small Molecule Neuroactive Compounds, Journal of the American Society for Mass Spectrometry, 2020, DOI: 10.1021/jasms.0c00166.

112. L. R. S. Huizing, S. R. Ellis, B. W. A. M. M. Beulen, F. P. Y. Barré, P. B. Kwant, R. J. Vreeken \& R. M. A. Heeren, Development and evaluation of matrix application techniques for high throughput mass spectrometry imaging of tissues in the clinic, Clinical Mass Spectrometry, 2019, 12, 7-15.

113. J. C. Vickerman, Impact of mass spectrometry in surface analysis, The Analyst, 1994, 119, 513523.

114. C.-C. Hsu \& P. C. Dorrestein, Visualizing Life With Ambient Mass Spectrometry, Current opinion in biotechnology, 2015, 0, 24-34.

115. C. Wu, A. L. Dill, L. S. Eberlin, R. G. Cooks \& D. R. Ifa, Mass spectrometry imaging under ambient conditions, Mass spectrometry reviews, 2013, 32, 218-243.

116. J. Xue, Y. Bai \& H. Liu, Recent advances in ambient mass spectrometry imaging, TrAC Trends in Analytical Chemistry, 2019, 120, 115659. 
117. Z. Takats, J. M. Wiseman \& R. G. Cooks, Ambient mass spectrometry using desorption electrospray ionization (DESI): instrumentation, mechanisms and applications in forensics, chemistry, and biology, Journal of Mass Spectrometry, 2005, 40, 1261-1275.

118. Z. Takáts, J. M. Wiseman, B. Gologan \& R. G. Cooks, Mass Spectrometry Sampling Under Ambient Conditions with Desorption Electrospray Ionization, Science (New York, N. Y.), 2004, 306, 471.

119. D. W. Etalo, C. Díez-Simón, R. C. H. de Vos \& R. D. Hall, Laser Ablation Electrospray lonizationMass Spectrometry Imaging (LAESI-MS) for Spatially Resolved Plant Metabolomics, Methods in Molecular Biology, 2018, 1778, 253-267.

120. P. Nemes \& A. Vertes, Laser Ablation Electrospray lonization for Atmospheric Pressure, in Vivo, and Imaging Mass Spectrometry, Analytical Chemistry, 2007, 79, 8098-8106.

121. R. L. Griffiths, A. J. Creese, A. M. Race, J. Bunch \& H. J. Cooper, LESA FAIMS Mass Spectrometry for the Spatial Profiling of Proteins from Tissue, Analytical Chemistry, 2016, 88, 6758-6766.

122. T. Porta, E. Varesio \& G. Hopfgartner, Gas-Phase Separation of Drugs and Metabolites Using Modifier-Assisted Differential Ion Mobility Spectrometry Hyphenated to Liquid Extraction Surface Analysis and Mass Spectrometry, Analytical Chemistry, 2013, 85, 11771-11779.

123. V. Kertesz \& G. J. Van Berkel, Liquid microjunction surface sampling coupled with high-pressure liquid chromatography-electrospray ionization-mass spectrometry for analysis of drugs and metabolites in whole-body thin tissue sections, Analytical Chemistry, 2010, 82, 5917-5921.

124. V. Kertesz \& G. J. Van Berkel, Sampling reliability, spatial resolution, spatial precision, and extraction efficiency in droplet-based liquid microjunction surface sampling, Rapid Communications in Mass Spectrometry, 2014, 28, 1553-1560.

125. G. J. Van Berkel, V. Kertesz, K. A. Koeplinger, M. Vavrek \& A. N. Kong, Liquid microjunction surface sampling probe electrospray mass spectrometry for detection of drugs and metabolites in thin tissue sections, Journal of Mass Spectrometry, 2008, 43, 500-508.

126. I. Lanekoff, K. Burnum-Johnson, M. Thomas, J. Short, J. P. Carson, J. Cha, S. K. Dey, P. Yang, M. C. Prieto Conaway \& J. Laskin, High-Speed Tandem Mass Spectrometric in Situ Imaging by Nanospray Desorption Electrospray lonization Mass Spectrometry, Analytical Chemistry, 2013, 85, 9596-9603.

127. J. Laskin, P. A. Eckert, P. J. Roach, B. S. Heath, S. A. Nizkorodov \& A. Laskin, Chemical Analysis of Complex Organic Mixtures Using Reactive Nanospray Desorption Electrospray lonization Mass Spectrometry, Analytical Chemistry, 2012, 84, 7179-7187.

128. M. Lorenz, O. S. Ovchinnikova \& G. J. Van Berkel, Fully automated laser ablation liquid capture surface analysis using nanoelectrospray ionization mass spectrometry, Rapid Communications in Mass Spectrometry, 2014, 28, 1312-1320.

129. J. I. Brauer, I. B. Beech \& J. Sunner, Mass Spectrometric Imaging Using Laser Ablation and Solvent Capture by Aspiration (LASCA), Journal of the American Society for Mass Spectrometry, 2015, 26, 1538-1547.

130. J. Laskin \& I. Lanekoff, Ambient Mass Spectrometry Imaging Using Direct Liquid Extraction Techniques, Analytical Chemistry, 2016, 88, 52-73.

131. L. S. Eberlin, X. Liu, C. R. Ferreira, S. Santagata, N. Y. R. Agar \& R. G. Cooks, Desorption Electrospray Ionization then MALDI Mass Spectrometry Imaging of Lipid and Protein Distributions in Single Tissue Sections, Analytical Chemistry, 2011, 83, 8366-8371.

132. J. Tillner, V. Wu, E. A. Jones, S. D. Pringle, T. Karancsi, A. Dannhorn, K. Veselkov, J. S. McKenzie \& Z. Takats, Faster, More Reproducible DESI-MS for Biological Tissue Imaging, Journal of The American Society for Mass Spectrometry, 2017, 28, 2090-2098.

133. C. M. Alfaro, V. Pirro, M. F. Keating, E. M. Hattab, R. G. Cooks \& A. A. Cohen-Gadol, Intraoperative assessment of isocitrate dehydrogenase mutation status in human gliomas using desorption electrospray ionization-mass spectrometry, Journal of neurosurgery, 2019, 132, 1-8.

134. V. Pirro, C. M. Alfaro, A. K. Jarmusch, E. M. Hattab, A. A. Cohen-Gadol \& R. G. Cooks, Intraoperative assessment of tumor margins during glioma resection by desorption electrospray ionization-mass spectrometry, Proceedings of the National Academy of Sciences, 2017, 114, 6700.

135. V. Kertesz \& G. J. Van Berkel, Fully automated liquid extraction-based surface sampling and ionization using a chip-based robotic nanoelectrospray platform, Journal of Mass Spectrometry, 2010, 45, 252-260

136. D. Eikel, M. Vavrek, S. Smith, C. Bason, S. Yeh, W. A. Korfmacher \& J. D. Henion, Liquid extraction surface analysis mass spectrometry (LESA-MS) as a novel profiling tool for drug distribution and metabolism analysis: the terfenadine example, Rapid Communications in Mass Spectrometry, 2011, 25, 3587-3596. 

Integration of Ion Mobility MS(E) after Fully Automated, Online, High-Resolution Liquid Extraction Surface Analysis Micro-Liquid Chromatography, Analytical Chemistry, 2017, 89, 11143-11150.

138. P. Vallance \& T. G. Smart, The future of pharmacology, British Journal of Pharmacology, 2006, 147, S304-S307.

139. M. Rudin \& R. Weissleder, Molecular imaging in drug discovery and development, Nature Reviews Drug Discovery, 2003, 2, 123-131.

140. L. Zhang, Y. Wang, D. Li, C. T. Ho, J. Li \& X. Wan, The absorption, distribution, metabolism and excretion of procyanidins, Food \& function, 2016, 7, 1273-1281.

141. Y. Sugihara, K. Watanabe \& A. Vegvari, Novel insights in drug metabolism by MS imaging, Bioanalysis, 2016, 8, 575-588.

142. J. G. Swales, G. Hamm, M. R. Clench \& R. J. A. Goodwin, Mass spectrometry imaging and its application in pharmaceutical research and development: A concise review, International journal of mass spectrometry, 2018, 437, 99-112.

143. A. Chacon, I. Zagol-Ikapitte, V. Amarnath, M. L. Reyzer, J. A. Oates, R. M. Caprioli \& O. Boutaud, On-tissue chemical derivatization of 3-methoxysalicylamine for MALDI-imaging mass spectrometry, Journal of Mass Spectrometry, 2011, 46, 840-846.

144. M. Shariatgorji, N. Strittmatter, A. Nilsson, P. Kallback, A. Alvarsson, X. Zhang, T. Vallianatou, P. Svenningsson, R. J. Goodwin \& P. E. Andren, Simultaneous imaging of multiple neurotransmitters and neuroactive substances in the brain by desorption electrospray ionization mass spectrometry, Neurolmage, 2016, 136, 129-138.

145. S. J. Atkinson, P. M. Loadman, C. Sutton, L. H. Patterson \& M. R. Clench, Examination of the distribution of the bioreductive drug AQ4N and its active metabolite AQ4 in solid tumours by imaging matrix-assisted laser desorption/ionisation mass spectrometry, Rapid Communications in Mass Spectrometry, 2007, 21, 1271-1276.

146. S. Ashton, Y. H. Song, J. Nolan, E. Cadogan, J. Murray, R. Odedra, J. Foster, P. A. Hall, S. Low, P. Taylor, R. Ellston, U. M. Polanska, J. Wilson, C. Howes, A. Smith, R. J. Goodwin, J. G. Swales, N. Strittmatter, Z. Takats, A. Nilsson, P. Andren, D. Trueman, M. Walker, C. L. Reimer, G. Troiano, D. Parsons, D. De Witt, M. Ashford, J. Hrkach, S. Zale, P. J. Jewsbury \& S. T. Barry, Aurora kinase inhibitor nanoparticles target tumors with favorable therapeutic index in vivo, Science translational medicine, 2016, 8, 325ra317.

147. R. J. A. Goodwin, A. Nilsson, C. L. Mackay, J. G. Swales, M. K. Johansson, M. Billger, P. E. Andrén \& S. L. Iverson, Exemplifying the Screening Power of Mass Spectrometry Imaging over Label-Based Technologies for Simultaneous Monitoring of Drug and Metabolite Distributions in Tissue Sections, Journal of Biomolecular Screening, 2015, 21, 187-193.

148. J. G. Swales, J. W. Tucker, M. J. Spreadborough, S. L. Iverson, M. R. Clench, P. J. Webborn \& R. J. Goodwin, Mapping drug distribution in brain tissue using liquid extraction surface analysis mass spectrometry imaging, Analytical Chemistry, 2015, 87, 10146-10152.

149. D. M. Drexler, T. J. Garrett, J. L. Cantone, R. W. Diters, J. G. Mitroka, M. C. Prieto Conaway, S. P. Adams, R. A. Yost \& M. Sanders, Utility of imaging mass spectrometry (IMS) by matrix-assisted laser desorption ionization (MALDI) on an ion trap mass spectrometer in the analysis of drugs and metabolites in biological tissues, Journal of pharmacological and toxicological methods, 2007, 55, 279-288.

150. K. J. Grove, V. Kansara, M. Prentiss, D. Long, M. Mogi, S. Kim \& P. J. Rudewicz, Application of Imaging Mass Spectrometry to Assess Ocular Drug Transit, SLAS Discovery, 2017, 22, 1239-1245.

151. H. Y. Wang, S. N. Jackson, J. McEuen \& A. S. Woods, Localization and analyses of small drug molecules in rat brain tissue sections, Analytical Chemistry, 2005, 77, 6682-6686.

152. Y. Yamada, K. Hidefumi, H. Shion, M. Oshikata \& Y. Haramaki, Distribution of chloroquine in ocular tissue of pigmented rat using matrix-assisted laser desorption/ionization imaging quadrupole timeof-flight tandem mass spectrometry, Rapid Communications in Mass Spectrometry, 2011, 25, 1600-1608.

153. W. B. Parson, S. L. Koeniger, R. W. Johnson, J. Erickson, Y. Tian, C. Stedman, A. Schwartz, E. Tarcsa, R. Cole \& G. J. Van Berkel, Analysis of chloroquine and metabolites directly from wholebody animal tissue sections by liquid extraction surface analysis (LESA) and tandem mass spectrometry, Journal of Mass Spectrometry, 2012, 47, 1420-1428.

154. M. Shariatgorji, P. Kallback, L. Gustavsson, N. Schintu, P. Svenningsson, R. J. Goodwin \& P. E. Andren, Controlled-pH tissue cleanup protocol for signal enhancement of small molecule drugs analyzed by MALDI-MS imaging, Analytical Chemistry, 2012, 84, 4603-4607. 
155. J. G. Swales, J. W. Tucker, N. Strittmatter, A. Nilsson, D. Cobice, M. R. Clench, C. L. Mackay, P. E. Andren, Z. Takats, P. J. Webborn \& R. J. Goodwin, Mass spectrometry imaging of cassette-dosed drugs for higher throughput pharmacokinetic and biodistribution analysis, Analytical Chemistry, 2014, 86, 8473-8480.

156. Y. Hsieh, R. Casale, E. Fukuda, J. Chen, I. Knemeyer, J. Wingate, R. Morrison \& W. Korfmacher, Matrix-assisted laser desorption/ionization imaging mass spectrometry for direct measurement of clozapine in rat brain tissue, Rapid Communications in Mass Spectrometry, 2006, 20, 965-972.

157. E. F. Choo, J. Ly, J. Chan, S. K. Shahidi-Latham, K. Messick, E. Plise, C. M. Quiason \& L. Yang, Role of P-glycoprotein on the brain penetration and brain pharmacodynamic activity of the MEK inhibitor cobimetinib, Molecular pharmaceutics, 2014, 11, 4199-4207.

158. J. Mains, C. Wilson \& A. Urquhart, ToF-SIMS analysis of ocular tissues reveals biochemical differentiation and drug distribution, European Journal of Pharmaceutics and Biopharmaceutics, 2011, 79, 328-333.

159. J. Mains, C. G. Wilson \& A. Urquhart, ToF-SIMS Analysis of Dexamethasone Distribution in the Isolated Perfused Eye, Investigative Ophthalmology \& Visual Science, 2011, 52, 8413-8419.

160. T. Matsumoto, H. Kushida, S. Matsushita, Y. Oyama, T. Suda, J. Watanabe, Y. Kase \& M. Setou, Distribution Analysis via Mass Spectrometry Imaging of Ephedrine in the Lungs of Rats Orally Administered the Japanese Kampo Medicine Maoto, Scientific reports, 2017, 7, 44098.

161. L. Signor, E. Varesio, R. F. Staack, V. Starke, W. F. Richter \& G. Hopfgartner, Analysis of erlotinib and its metabolites in rat tissue sections by MALDI quadrupole time-of-flight mass spectrometry, Journal of Mass Spectrometry, 2007, 42, 900-909.

162. S. Castellino, M. R. Groseclose, J. Sigafoos, D. Wagner, M. de Serres, J. W. Polli, E. Romach, J. Myer \& B. Hamilton, Central nervous system disposition and metabolism of Fosdevirine (GSK2248761), a non-nucleoside reverse transcriptase inhibitor: an LC-MS and Matrix-assisted laser desorption/ionization imaging MS investigation into central nervous system toxicity, Chemical research in toxicology, 2013, 26, 241-251.

163. G. Marko-Varga, T. E. Fehniger, M. Rezeli, B. Dome, T. Laurell \& A. Vegvari, Drug localization in different lung cancer phenotypes by MALDI mass spectrometry imaging, Journal of Proteomics, 2011, 74, 982-992.

164. N. S. Nagathihalli, J. A. Castellanos, C. Shi, Y. Beesetty, M. L. Reyzer, R. Caprioli, X. Chen, A. J. Walsh, M. C. Skala, H. L. Moses \& N. B. Merchant, Signal Transducer and Activator of Transcription 3, Mediated Remodeling of the Tumor Microenvironment Results in Enhanced Tumor Drug Delivery in a Mouse Model of Pancreatic Cancer, Gastroenterology, 2015, 149, 1932-1943.

165. P. Marshall, V. Toteu-Djomte, P. Bareille, H. Perry, G. Brown, M. Baumert \& K. Biggadike, Correlation of skin blanching and percutaneous absorption for glucocorticoid receptor agonists by matrix-assisted laser desorption ionization mass spectrometry imaging and liquid extraction surface analysis with nanoelectrospray ionization mass spectrometry, Analytical Chemistry, 2010, 82, 7787-7794.

166. A. Rompp, S. Guenther, Z. Takats \& B. Spengler, Mass spectrometry imaging with high resolution in mass and space (HR(2) MSI) for reliable investigation of drug compound distributions on the cellular level, Analytical and Bioanalytical Chemistry, 2011, 401, 65-73.

167. T. E. Fehniger, Á. Végvári, M. Rezeli, K. Prikk, P. Ross, M. Dahlbäck, G. Edula, R. Sepper \& G. Marko-Varga, Direct Demonstration of Tissue Uptake of an Inhaled Drug: Proof-of-Principle Study Using Matrix-Assisted Laser Desorption Ionization Mass Spectrometry Imaging, Analytical Chemistry, 2011, 83, 8329-8336.

168. M. L. Manier, M. L. Reyzer, A. Goh, V. Dartois, L. E. Via, C. E. Barry, 3rd \& R. M. Caprioli, Reagent precoated targets for rapid in-tissue derivatization of the anti-tuberculosis drug isoniazid followed by MALDI imaging mass spectrometry, Journal of the American Society for Mass Spectrometry, 2011, 22, 1409-1419.

169. J. Bunch, M. R. Clench \& D. S. Richards, Determination of pharmaceutical compounds in skin by imaging matrix-assisted laser desorption/ionisation mass spectrometry, Rapid Communications in Mass Spectrometry, 2004, 18, 3051-3060.

170. S. Castellino, M. R. Groseclose \& D. Wagner, MALDI imaging mass spectrometry: bridging biology and chemistry in drug development, Bioanalysis, 2011, 3, 2427-2441.

171. J. D'Alvise, R. Mortensen, S. H. Hansen \& C. Janfelt, Detection of follicular transport of lidocaine and metabolism in adipose tissue in pig ear skin by DESI mass spectrometry imaging, Analytical and Bioanalytical Chemistry, 2014, 406, 3735-3742.

172. Y. G. Shin, T. Dong, B. Chou \& K. Menghrajani, Determination of loperamide in Mdr1a/1b knockout mouse brain tissue using matrix-assisted laser desorption/ionization mass spectrometry and 
comparison with quantitative electrospray-triple quadrupole mass spectrometry analysis, Archives of Pharmacal Research, 2011, 34, 1983-1988.

173. B. Prideaux, V. Dartois, D. Staab, D. M. Weiner, A. Goh, L. E. Via, C. E. Barry \& M. Stoeckli, HighSensitivity MALDI-MRM-MS Imaging of Moxifloxacin Distribution in Tuberculosis-Infected Rabbit Lungs and Granulomatous Lesions, Analytical Chemistry, 2011, 83, 2112-2118.

174. L. J. Dekker, J. J. van Kampen, M. L. Reedijk, P. C. Burgers, R. A. Gruters, A. D. Osterhaus \& T. M. Luider, A mass spectrometry based imaging method developed for the intracellular detection of HIV protease inhibitors, Rapid Communications in Mass Spectrometry, 2009, 23, 1183-1188.

175. L. Morosi, C. Matteo, T. Ceruti, S. Giordano, M. Ponzo, R. Frapolli, M. Zucchetti, E. Davoli, M. D'Incalci \& P. Ubezio, Quantitative determination of niraparib and olaparib tumor distribution by mass spectrometry imaging, International Journal of Biological Sciences, 2020, 16, 1363-1375.

176. J. G. Swales, N. Strittmatter, J. W. Tucker, M. R. Clench, P. J. H. Webborn \& R. J. A. Goodwin, Spatial Quantitation of Drugs in tissues using Liquid Extraction Surface Analysis Mass Spectrometry Imaging, Scientific reports, 2016, 6, 37648.

177. S. L. Koeniger, N. Talaty, Y. Luo, D. Ready, M. Voorbach, T. Seifert, S. Cepa, J. A. Fagerland, J. Bouska, W. Buck, R. W. Johnson \& S. Spanton, A quantitation method for mass spectrometry imaging, Rapid Communications in Mass Spectrometry, 2011, 25, 503-510.

178. A. Bouslimani, N. Bec, M. Glueckmann, C. Hirtz \& C. Larroque, Matrix-assisted laser desorption/ionization imaging mass spectrometry of oxaliplatin derivatives in heated intraoperative chemotherapy (HIPEC)-like treated rat kidney, Rapid Communications in Mass Spectrometry, 2010, 24, 415-421.

179. F. J. Troendle, C. D. Reddick \& R. A. Yost, Detection of pharmaceutical compounds in tissue by matrix-assisted laser desorption/ionization and laser desorption/chemical ionization tandem mass spectrometry with a quadrupole ion trap, Journal of the American Society for Mass Spectrometry, 1999, 10, 1315-1321.

180. S. Giordano, L. Morosi, P. Veglianese, S. A. Licandro, R. Frapolli, M. Zucchetti, G. Cappelletti, L. Falciola, V. Pifferi, S. Visentin, M. D'Incalci \& E. Davoli, 3D Mass Spectrometry Imaging Reveals a Very Heterogeneous Drug Distribution in Tumors, Scientific reports, 2016, 6, 37027-37027.

181. J. Zhang, Q. Du, X. Song, S. Gao, X. Pang, Y. Li, R. Zhang, Z. Abliz \& J. He, Evaluation of the tumortargeting efficiency and intratumor heterogeneity of anticancer drugs using quantitative mass spectrometry imaging, Theranostics, 2020, 10, 2621-2630.

182. L. Salphati, S. Shahidi-Latham, C. Quiason, K. Barck, M. Nishimura, B. Alicke, J. Pang, R. A. Carano, A. G. Olivero \& H. S. Phillips, Distribution of the phosphatidylinositol 3-kinase inhibitors Pictilisib (GDC-0941) and GNE-317 in U87 and GS2 intracranial glioblastoma models-assessment by matrixassisted laser desorption ionization imaging, Drug Metabolism and Disposition, 2014, 42, 11101116.

183. N. Sun, I. E. Fernandez, M. Wei, Y. Wu, M. Aichler, O. Eickelberg \& A. Walch, Pharmacokinetic and pharmacometabolomic study of pirfenidone in normal mouse tissues using high mass resolution MALDI-FTICR-mass spectrometry imaging, Histochemistry and Cell Biology, 2016, 145, 201-211.

184. A. Nilsson, R. J. Goodwin, J. G. Swales, R. Gallagher, H. Shankaran, A. Sathe, S. Pradeepan, A. Xue, N. Keirstead, J. C. Sasaki, P. E. Andren \& A. Gupta, Investigating nephrotoxicity of polymyxin derivatives by mapping renal distribution using mass spectrometry imaging, Chemical research in toxicology, 2015, 28, 1823-1830.

185. R. A. McClure, C. W. Chumbley, M. L. Reyzer, K. Wilson, R. M. Caprioli, J. C. Gore \& W. Pham, Identification of promethazine as an amyloid-binding molecule using a fluorescence highthroughput assay and MALDI imaging mass spectrometry, Neuroimage: Clinical, 2013, 2, 620-629.

186. V. Kertesz, G. J. Van Berkel, M. Vavrek, K. A. Koeplinger, B. B. Schneider \& T. R. Covey, Comparison of drug distribution images from whole-body thin tissue sections obtained using desorption electrospray ionization tandem mass spectrometry and autoradiography, Analytical Chemistry, 2008, 80, 5168-5177.

187. R. J. A. Goodwin, C. L. Mackay, A. Nilsson, D. J. Harrison, L. Farde, P. E. Andren \& S. L. Iverson, Qualitative and Quantitative MALDI Imaging of the Positron Emission Tomography Ligands Raclopride (a D2 Dopamine Antagonist) and SCH 23390 (a D1 Dopamine Antagonist) in Rat Brain Tissue Sections Using a Solvent-Free Dry Matrix Application Method, Analytical Chemistry, 2011, 83, 9694-9701.

188. N. Takai, Y. Tanaka, K. Inazawa \& H. Saji, Quantitative analysis of pharmaceutical drug distribution in multiple organs by imaging mass spectrometry, Rapid Communications in Mass Spectrometry, 2012, 26, 1549-1556. 
189. S. K. Shahidi-Latham, S. M. Dutta, M. C. Prieto Conaway \& P. J. Rudewicz, Evaluation of an Accurate Mass Approach for the Simultaneous Detection of Drug and Metabolite Distributions via Whole-Body Mass Spectrometric Imaging, Analytical Chemistry, 2012, 84, 7158-7165.

190. C. W. Chumbley, M. L. Reyzer, J. L. Allen, G. A. Marriner, L. E. Via, C. E. Barry, 3rd \& R. M. Caprioli, Absolute Quantitative MALDI Imaging Mass Spectrometry: A Case of Rifampicin in Liver Tissues, Analytical Chemistry, 2016, 88, 2392-2398.

191. N. Takai, Y. Tanaka \& H. Saji, Quantification of small molecule drugs in biological tissue sections by imaging mass spectrometry using surrogate tissue-based calibration standards, Mass Spectrometry (Tokyo), 2014, 3, A0025.

192. G. R. Hamm, E. Bäckström, M. Brülls, A. Nilsson, N. Strittmatter, P. E. Andrén, K. Grime, M. Fridén \& R. J. A. Goodwin, Revealing the Regional Localization and Differential Lung Retention of Inhaled Compounds by Mass Spectrometry Imaging, Journal of aerosol medicine and pulmonary drug delivery, 2020, 33, 43-53.

193. M. L. Reyzer, Y. Hsieh, K. Ng, W. A. Korfmacher \& R. M. Caprioli, Direct analysis of drug candidates in tissue by matrix-assisted laser desorption/ionization mass spectrometry, Journal of Mass Spectrometry, 2003, 38, 1081-1092.

194. S. M. Boudon, G. Morandi, B. Prideaux, D. Staab, U. Junker, A. Odermatt, M. Stoeckli \& D. Bauer Evaluation of sparfloxacin distribution by mass spectrometry imaging in a phototoxicity model, Journal of the American Society for Mass Spectrometry, 2014, 25, 1803-1809.

195. J. Chen, Y. Hsieh, I. Knemeyer, L. Crossman \& W. A. Korfmacher, Visualization of first-pass drug metabolism of terfenadine by MALDI-imaging mass spectrometry, Drug metabolism letters, 2008, 2, 1-4.

196. A. Nilsson, T. E. Fehniger, L. Gustavsson, M. Andersson, K. Kenne, G. Marko-Varga \& P. E. Andren, Fine mapping the spatial distribution and concentration of unlabeled drugs within tissue microcompartments using imaging mass spectrometry, PloS one, 2010, 5, e11411.

197. A. Vegvari, T. E. Fehniger, L. Gustavsson, A. Nilsson, P. E. Andren, K. Kenne, J. Nilsson, T. Laurell \& G. Marko-Varga, Essential tactics of tissue preparation and matrix nano-spotting for successful compound imaging mass spectrometry, Journal of Proteomics, 2010, 73, 1270-1278.

198. I. H. Bartelink, B. Prideaux, G. Krings, L. Wilmes, P. R. E. Lee, P. Bo, B. Hann, J. P. Coppe, D. Heditsian, L. Swigart-Brown, E. F. Jones, S. Magnitsky, R. J. Keizer, N. de Vries, H. Rosing, N. Pawlowska, S. Thomas, M. Dhawan, R. Aggarwal, P. N. Munster, L. J. Esserman, W. Ruan, A. H. B. Wu, D. Yee, V. Dartois, R. M. Savic, D. M. Wolf \& L. van 't Veer, Heterogeneous drug penetrance of veliparib and carboplatin measured in triple negative breast tumors, Breast Cancer Research, 2017, 19, 107.

199. P. J. Trim, C. M. Henson, J. L. Avery, A. McEwen, M. F. Snel, E. Claude, P. S. Marshall, A. West, A. P. Princivalle \& M. R. Clench, Matrix-assisted laser desorption/ionization-ion mobility separationmass spectrometry imaging of vinblastine in whole body tissue sections, Analytical Chemistry, 2008, 80, 8628-8634.

200. M. Stoeckli, D. Staab, A. Schweitzer, J. Gardiner \& D. Seebach, Imaging of a beta-peptide distribution in whole-body mice sections by MALDI mass spectrometry, Journal of the American Society for Mass Spectrometry, 2007, 18, 1921-1924.

201. S. R. Ellis, A. L. Bruinen \& R. M. A. Heeren, A critical evaluation of the current state-of-the-art in quantitative imaging mass spectrometry, Analytical and Bioanalytical Chemistry, 2013, 406, 12751289.

202. A. Taylor, A. Dexter \& J. Bunch, Exploring ion suppression in mass spectrometry imaging of a heterogeneous tissue, Analytical Chemistry, 2018, 90, 5637-5645.

203. T. Porta, A. Lesur, E. Varesio \& G. Hopfgartner, Quantification in MALDI-MS imaging: what can we learn from MALDI-selected reaction monitoring and what can we expect for imaging?, Analytical and Bioanalytical Chemistry, 2015, 407, 2177-2187.

204. D. A. Pirman, A. Kiss, R. M. Heeren \& R. A. Yost, Identifying tissue-specific signal variation in MALDI mass spectrometric imaging by use of an internal standard, Analytical Chemistry, 2013, 85, 1090 1096.

205. D. A. Pirman, R. F. Reich, A. Kiss, R. M. Heeren \& R. A. Yost, Quantitative MALDI tandem mass spectrometric imaging of cocaine from brain tissue with a deuterated internal standard, Analytical Chemistry, 2013, 85, 1081-1089.

206. M. R. Groseclose \& S. Castellino, A Mimetic Tissue Model for the Quantification of Drug Distributions by MALDI Imaging Mass Spectrometry, Analytical Chemistry, 2013, 85, 1009910106. 

Model for Quantitative Imaging Mass Spectrometry, Protocol Exchange, 2018.

208. G. Hamm, D. Bonnel, R. Legouffe, F. Pamelard, J. M. Delbos, F. Bouzom \& J. Stauber, Quantitative mass spectrometry imaging of propranolol and olanzapine using tissue extinction calculation as normalization factor, Journal of Proteomics, 2012, 75, 4952-4961.

209. R. D. Addie, B. Balluff, J. V. M. G. Bovée, H. Morreau \& L. A. McDonnell, Current State and Future Challenges of Mass Spectrometry Imaging for Clinical Research, Analytical Chemistry, 2015, 87, 6426-6433.

210. B. Balluff, C. K. Frese, S. K. Maier, C. Schöne, B. Kuster, M. Schmitt, M. Aubele, H. Höfler, A. M. Deelder, A. J. Heck, P. C. Hogendoorn, J. Morreau, A. Maarten Altelaar, A. Walch \& L. A. McDonnell, De novo discovery of phenotypic intratumour heterogeneity using imaging mass spectrometry, Journal of Pathology, 2015, 235, 3-13.

211. H. Ye, E. Gemperline \& L. Li, A vision for better health: mass spectrometry imaging for clinical diagnostics, Clinica Chimica Acta, 2013, 420, 11-22.

212. R. Longuespée, R. Casadonte, M. Kriegsmann, C. Pottier, G. Picard de Muller, P. Delvenne, J. Kriegsmann \& E. De Pauw, MALDI mass spectrometry imaging: A cutting-edge tool for fundamental and clinical histopathology, Proteomics Clinical Applications, 2016, 10, 701-719.

213. H. J. Kwon, Y. Kim, Y. Sugihara, B. Baldetorp, C. Welinder, K.-i. Watanabe, T. Nishimura, J. Malm, S. Török, B. Döme, Á. Végvári, L. Gustavsson, T. E. Fehniger \& G. Marko-Varga, Drug compound characterization by mass spectrometry imaging in cancer tissue, Archives of pharmacal research, 2015, 38, 1718-1727.

214. A. C. Crecelius, U. S. Schubert \& F. von Eggeling, MALDI mass spectrometric imaging meets "omics": recent advances in the fruitful marriage, The Analyst, 2015, 140, 5806-5820.

215. L. M. Cole \& M. R. Clench, Mass spectrometry imaging tools in oncology, Biomark Med, 2015, 9, 863-868.

216. J. Hanrieder, P. Malmberg \& A. G. Ewing, Spatial neuroproteomics using imaging mass spectrometry, Biochim Biophys Acta, 2015, 1854, 718-731.

217. C. Mériaux, J. Franck, D. B. Park, J. Quanico, Y. H. Kim, C. K. Chung, Y. M. Park, H. Steinbusch, M. Salzet \& I. Fournier, Human temporal lobe epilepsy analyses by tissue proteomics, Hippocampus, 2014, 24, 628-642.

218. K. O. Schubert, F. Weiland, B. T. Baune \& P. Hoffmann, The use of MALDI-MSI in the investigation of psychiatric and neurodegenerative disorders: A review, Proteomics, 2016, 16, 1747-1758.

219. A. C. Dona, S. Coffey \& G. Figtree, Translational and emerging clinical applications of metabolomics in cardiovascular disease diagnosis and treatment, European Journal of Preventive Cardiology, 2016, 23, 1578-1589.

220. M. Martin-Lorenzo, G. Alvarez-Llamas, L. A. McDonnell \& F. Vivanco, Molecular histology of arteries: mass spectrometry imaging as a novel ex vivo tool to investigate atherosclerosis, Expert Reviews in Proteomics, 2016, 13, 69-81.

221. H. E. Bowrey, D. M. Anderson, P. Pallitto, D. B. Gutierrez, J. Fan, R. K. Crouch, K. L. Schey \& Z. Ablonczy, Imaging mass spectrometry of the visual system: Advancing the molecular understanding of retina degenerations, Proteomics. Clinical applications, 2016, 10, 391-402.

222. B. Cillero-Pastor, G. B. Eijkel, F. J. Blanco \& R. M. A. Heeren, Protein classification and distribution in osteoarthritic human synovial tissue by matrix-assisted laser desorption ionization mass spectrometry imaging, Analytical and Bioanalytical Chemistry, 2015, 407, 2213-2222.

223. S. R. Ellis, J. Cappell, N. O. Potocnik, B. Balluff, J. Hamaide, A. Van der Linden \& R. M. A. Heeren, More from less: high-throughput dual polarity lipid imaging of biological tissues, The Analyst, 2016, 141, 3832-3841.

224. N. Ogrinc Potocnik, T. Porta, M. Becker, R. M. Heeren \& S. R. Ellis, Use of advantageous, volatile matrices enabled by next-generation high-speed matrix-assisted laser desorption/ionization timeof-flight imaging employing a scanning laser beam, Rapid Communications in Mass Spectrometry, 2015, 29, 2195-2203.

225. J. M. Spraggins, D. G. Rizzo, J. L. Moore, M. J. Noto, E. P. Skaar \& R. M. Caprioli, Next-generation technologies for spatial proteomics: Integrating ultra-high speed MALDI-TOF and high mass resolution MALDI FTICR imaging mass spectrometry for protein analysis, Proteomics, 2016, 16, 1678-1689.

226. D. R. Ifa \& L. S. Eberlin, Ambient Ionization Mass Spectrometry for Cancer Diagnosis and Surgical Margin Evaluation, Clinical chemistry, 2016, 62, 111-123.

227. E. C. Cabral \& D. R. Ifa, DESI imaging of small molecules in biological tissues, Methods in molecular biology (Clifton, N.J.), 2015, 1203, 63-77. 
228. K. Skraskova, E. Claude, E. A. Jones, M. Towers, S. R. Ellis \& R. M. Heeren, Enhanced capabilities for imaging gangliosides in murine brain with matrix-assisted laser desorption/ionization and desorption electrospray ionization mass spectrometry coupled to ion mobility separation, Methods, 2016, 104, 69-78.

229. N. Abbassi-Ghadi, E. A. Jones, M. Gomez-Romero, O. Golf, S. Kumar, J. Huang, H. Kudo, R. D. Goldin, G. B. Hanna \& Z. Takats, A Comparison of DESI-MS and LC-MS for the Lipidomic Profiling of Human Cancer Tissue, Journal of the American Society for Mass Spectrometry, 2016, 27, 255264.

230. C. M. Alfaro, A. K. Jarmusch, V. Pirro, K. S. Kerian, T. A. Masterson, L. Cheng \& R. G. Cooks, Ambient ionization mass spectrometric analysis of human surgical specimens to distinguish renal cell carcinoma from healthy renal tissue, Analytical and Bioanalytical Chemistry, 2016, 408, 5407 5414.

231. L. S. Eberlin, I. Norton, D. Orringer, I. F. Dunn, X. Liu, J. L. Ide, A. K. Jarmusch, K. L. Ligon, F. A. Jolesz, A. J. Golby, S. Santagata, N. Y. R. Agar \& R. G. Cooks, Ambient mass spectrometry for the intraoperative molecular diagnosis of human brain tumors, Proceedings of the National Academy of Sciences of the United States of America, 2013, 110, 1611-1616.

232. S. Gerbig, O. Golf, J. Balog, J. Denes, Z. Baranyai, A. Zarand, E. Raso, J. Timar \& Z. Takats, Analysis of colorectal adenocarcinoma tissue by desorption electrospray ionization mass spectrometric imaging, Analytical and Bioanalytical Chemistry, 2012, 403, 2315-2325.

233. A. K. Jarmusch, V. Pirro, Z. Baird, E. M. Hattab, A. A. Cohen-Gadol \& R. G. Cooks, Lipid and metabolite profiles of human brain tumors by desorption electrospray ionization-MS, Proceedings of the National Academy of Sciences of the United States of America, 2016, 113, 1486-1491.

234. J. Zhang, C. L. Feider, C. Nagi, W. Yu, S. A. Carter, J. Suliburk, H. S. T. Cao \& L. S. Eberlin, Detection of Metastatic Breast and Thyroid Cancer in Lymph Nodes by Desorption Electrospray Ionization Mass Spectrometry Imaging, Journal of the American Society for Mass Spectrometry, 2017, 28, 1166-1174.

235. L. S. Eberlin, I. Norton, A. L. Dill, A. J. Golby, K. L. Ligon, S. Santagata, R. G. Cooks \& N. Y. Agar, Classifying human brain tumors by lipid imaging with mass spectrometry, Cancer research, 2012, 72, 645-654.

236. S. Schadt, S. Kallbach, R. Almeida \& J. Sandel, Investigation of Figopitant and Its Metabolites in Rat Tissue by Combining Whole-Body Autoradiography with Liquid Extraction Surface Analysis Mass Spectrometry, Drug Metabolism and Disposition, 2012, 40, 419-425.

237. E. C. Randall, J. Bunch \& H. J. Cooper, Direct Analysis of Intact Proteins from Escherichia coli Colonies by Liquid Extraction Surface Analysis Mass Spectrometry, Analytical Chemistry, 2014, 86, 10504-10510.

238. C. Lanshoeft, G. Stutz, W. Elbast, T. Wolf, M. Walles, M. Stoeckli, F. Picard \& O. Kretz, Analysis of small molecule antibody-drug conjugate catabolites in rat liver and tumor tissue by liquid extraction surface analysis micro-capillary liquid chromatography/tandem mass spectrometry, Rapid Communications in Mass Spectrometry, 2016, 30, 823-832.

239. R. Almeida, Z. Berzina, E. C. Arnspang, J. Baumgart, J. Vogt, R. Nitsch \& C. S. Ejsing, Quantitative spatial analysis of the mouse brain lipidome by pressurized liquid extraction surface analysis, Analytical Chemistry, 2015, 87, 1749-1756.

240. J. Quanico, J. Franck, C. Dauly, K. Strupat, J. Dupuy, R. Day, M. Salzet, I. Fournier \& M. Wisztorski, Development of liquid microjunction extraction strategy for improving protein identification from tissue sections, Journal of Proteomics, 2013, 79, 200-218.

241. M. Wisztorski, A. Desmons, J. Quanico, B. Fatou, J. P. Gimeno, J. Franck, M. Salzet \& I. Fournier, Spatially-resolved protein surface microsampling from tissue sections using liquid extraction surface analysis, Proteomics, 2016, 16, 1622-1632.

242. M. Wisztorski, B. Fatou, J. Franck, A. Desmons, I. Farré, E. Leblanc, I. Fournier \& M. Salzet, Microproteomics by liquid extraction surface analysis: application to FFPE tissue to study the fimbria region of tubo-ovarian cancer, Proteomics - Clinical Applications, 2013, 7, 234-240.

243. N. J. Martin, R. L. Griffiths, R. L. Edwards \& H. J. Cooper, Native Liquid Extraction Surface Analysis Mass Spectrometry: Analysis of Noncovalent Protein Complexes Directly from Dried Substrates, Journal of the American Society for Mass Spectrometry, 2015, 26, 1320-1327.

244. J. Sarsby, R. L. Griffiths, A. M. Race, J. Bunch, E. C. Randall, A. J. Creese \& H. J. Cooper, Liquid Extraction Surface Analysis Mass Spectrometry Coupled with Field Asymmetric Waveform Ion Mobility Spectrometry for Analysis of Intact Proteins from Biological Substrates, Analytical Chemistry, 2015, 87, 6794-6800. 
L. Tomlinson, J. Fuchser, A. Futterer, M. Baumert, D. G. Hassall, A. West \& P. S. Marshall, Using a single, high mass resolution mass spectrometry platform to investigate ion suppression effects observed during tissue imaging, Rapid Communications in Mass Spectrometry, 2014, 28, 9951003.

246. C. L. Feider, N. Elizondo \& L. S. Eberlin, Ambient Ionization and FAIMS Mass Spectrometry for Enhanced Imaging of Multiply Charged Molecular Ions in Biological Tissues, Analytical Chemistry, 2016, 88, 11533-11541.

247. V. Kertesz, T. M. Weiskittel \& G. J. Van Berkel, An enhanced droplet-based liquid microjunction surface sampling system coupled with HPLC-ESI-MS/MS for spatially resolved analysis, Analytical and Bioanalytical Chemistry, 2015, 407, 2117-2125.

248. V. Kertesz, D. Calligaris, D. R. Feldman, A. Changelian, E. R. Laws, S. Santagata, N. Y. Agar \& G. J. Van Berkel, Profiling of adrenocorticotropic hormone and arginine vasopressin in human pituitary gland and tumor thin tissue sections using droplet-based liquid-microjunction surface-samplingHPLC-ESI-MS-MS, Analytical and Bioanalytical Chemistry, 2015, 407, 5989-5998.

249. R. Almeida, J. Vogt, K. H. Hannibal-Bach, J. Baumgart, R. Nitsch \& C. Ejsing, Denver, 2011.

250. G. Z. Li, J. P. Vissers, J. C. Silva, D. Golick, M. V. Gorenstein \& S. J. Geromanos, Database searching and accounting of multiplexed precursor and product ion spectra from the data independent analysis of simple and complex peptide mixtures, Proteomics, 2009, 9, 1696-1719.

251. Y. Wu, E. G. Williams \& R. Aebersold, Application of SWATH Proteomics to Mouse Biology, Curr Protoc Mouse Biol, 2017, 7, 130-143.

252. U. Distler, J. Kuharev, P. Navarro, Y. Levin, H. Schild \& S. Tenzer, Drift time-specific collision energies enable deep-coverage data-independent acquisition proteomics, Nature Methods, 2014, 11, 167 170.

253. A. F. M. Altelaar, S. L. Luxembourg, L. A. McDonnell, S. R. Piersma \& R. M. A. Heeren, Imaging mass spectrometry at cellular length scales, Nature Protocols, 2007, 2, 1185-1196.

254. I. M. Taban, A. F. Altelaar, Y. E. van der Burgt, L. A. McDonnell, R. M. Heeren, J. Fuchser \& G. Baykut, Imaging of peptides in the rat brain using MALDI-FTICR mass spectrometry, Journal of the American Society for Mass Spectrometry, 2007, 18, 145-151.

255. G. Hao \& S. S. Gross, Electrospray tandem mass spectrometry analysis of S- and N-nitrosopeptides: Facile loss of NO and radical-induced fragmentation, Journal of The American Society for Mass Spectrometry, 2006, 17, 1725-1730.

256. E. Ricciotti \& G. A. FitzGerald, Prostaglandins and Inflammation, Arteriosclerosis, Thrombosis, and Vascular Biology, 2011, 31, 986-1000.

257. C. Nathan, Points of control in inflammation, Nature, 2002, 420, 846-852.

258. C. D. Funk, Prostaglandins and leukotrienes: advances in eicosanoid biology, Science (New York, N.Y.), 2001, 294, 1871-1875.

259. E. M. Smyth, T. Grosser, M. Wang, Y. Yu \& G. A. FitzGerald, Prostanoids in health and disease, Journal of Lipid Research, 2009, 50, S423-S428.

260. R. N. Dubois, S. B. Abramson, L. Crofford, R. A. Gupta, L. S. Simon, L. B. Van De Putte \& P. E. Lipsky, Cyclooxygenase in biology and disease, FASEB Journal, 1998, 12, 1063-1073.

261. W. L. Smith, D. L. DeWitt \& R. M. Garavito, Cyclooxygenases: structural, cellular, and molecular biology, Annual Review of Biochemistry, 2000, 69, 145-182.

262. J. Song, X. Liu, J. Wu, M. J. Meehan, J. M. Blevitt, P. C. Dorrestein \& M. E. Milla, A highly efficient, high-throughput lipidomics platform for the quantitative detection of eicosanoids in human whole blood, Analytical biochemistry, 2013, 433, 181-188.

263. K. Strassburg, A. M. Huijbrechts, K. A. Kortekaas, J. H. Lindeman, T. L. Pedersen, A. Dane, R. Berger, A. Brenkman, T. Hankemeier, J. van Duynhoven, E. Kalkhoven, J. W. Newman \& R. J. Vreeken, Quantitative profiling of oxylipins through comprehensive LC-MS/MS analysis: application in cardiac surgery, Analytical and Bioanalytical Chemistry, 2012, 404, 1413-1426.

264. Y. Wang, A. M. Armando, O. Quehenberger, C. Yan \& E. A. Dennis, Comprehensive ultraperformance liquid chromatographic separation and mass spectrometric analysis of eicosanoid metabolites in human samples, Journal of Chromatography A, 2014, 1359, 60-69.

265. A. J. Cartwright, P. Jones, J. C. Wolff \& E. H. Evans, Derivatisation of carboxylic acid groups in pharmaceuticals for enhanced detection using liquid chromatography with electrospray ionisation tandem mass spectrometry, Rapid Communications in Mass Spectrometry, 2005, 19, 1058-1062.

266. A. Kretschmer, M. Giera, M. Wijtmans, L. de Vries, H. Lingeman, H. Irth \& W. M. Niessen, Derivatization of carboxylic acids with 4-APEBA for detection by positive-ion LC-ESI-MS(/MS) applied for the analysis of prostanoids and NSAID in urine, Journal of Chromatography B, 2011, 879, 1393-1401. 
267. D. Kloos, H. Lingeman, O. A. Mayboroda, A. M. Deelder, W. M. A. Niessen \& M. Giera, Analysis of biologically-active, endogenous carboxylic acids based on chromatography-mass spectrometry, TrAC Trends in Analytical Chemistry, 2014, 61, 17-28.

268. R. Yang, N. Chiang, S. F. Oh \& C. N. Serhan, Metabolomics-lipidomics of eicosanoids and docosanoids generated by phagocytes, Current protocols in immunology, 2011, Chapter 14, Unit 1426.

269. Z. Chen, M. S. Glover \& L. Li, Recent advances in ion mobility-mass spectrometry for improved structural characterization of glycans and glycoconjugates, Current opinion in chemical biology, 2017, 42, 1-8.

270. L. S. Fenn, M. Kliman, A. Mahsut, S. R. Zhao \& J. A. McLean, Characterizing ion mobility-mass spectrometry conformation space for the analysis of complex biological samples, Analytical and Bioanalytical Chemistry, 2009, 394, 235-244.

271. J. D. Keelor, P. Dwivedi \& F. M. Fernandez, An effective approach for coupling direct analysis in real time with atmospheric pressure drift tube ion mobility spectrometry, Journal of the American Society for Mass Spectrometry, 2014, 25, 1538-1548.

272. J. C. May, C. R. Goodwin, N. M. Lareau, K. L. Leaptrot, C. B. Morris, R. T. Kurulugama, A. Mordehai, C. Klein, W. Barry, E. Darland, G. Overney, K. Imatani, G. C. Stafford, J. C. Fjeldsted \& J. A. McLean, Conformational ordering of biomolecules in the gas phase: nitrogen collision cross sections measured on a prototype high resolution drift tube ion mobility-mass spectrometer, Analytical Chemistry, 2014, 86, 2107-2116.

273. C. S. Maier, F. Zandkarimi, S. Wickramasekara, J. Morre \& J. F. Stevens, in 50 Years of Phytochemistry Research: Volume 43, ed. D. R. Gang, Springer International Publishing, Cham, 2013, DOI: 10.1007/978-3-319-00581-2 2, pp. 21-41.

274. S. J. Allen, K. Giles, T. Gilbert \& M. F. Bush, lon mobility mass spectrometry of peptide, protein, and protein complex ions using a radio-frequency confining drift cell, The Analyst, 2016, 141, 884891.

275. P. Dwivedi, A. J. Schultz \& H. H. Hill, Metabolic Profiling of Human Blood by High Resolution Ion Mobility Mass Spectrometry (IM-MS), International journal of mass spectrometry, 2010, 298, 7890.

276. P. Dwivedi, P. Wu, S. J. Klopsch, G. J. Puzon, L. Xun \& H. H. Hill, Metabolic profiling by ion mobility mass spectrometry (IMMS), Metabolomics, 2008, 4, 63-80.

277. A. A. Shvartsburg, T. A. Seim, W. F. Danielson, R. Norheim, R. J. Moore, G. A. Anderson \& R. D. Smith, High-Definition Differential Ion Mobility Spectrometry with Resolving Power up to 500, Journal of the American Society for Mass Spectrometry, 2013, 24, 109-114.

278. K. Giles, J. Ujma, J. Wildgoose, S. Pringle, K. Richardson, D. Langridge \& M. Green, A Cyclic Ion Mobility-Mass Spectrometry System, Analytical Chemistry, 2019, 91, 8564-8573.

279. E. Riches \& M. E. Palmer, Application of a novel cyclic ion mobility-mass spectrometer to the analysis of synthetic polymers: A preliminary evaluation, Rapid Communications in Mass Spectrometry, n/a.

280. T. Kenderdine, R. Nemati, A. Baker, M. Palmer, J. Ujma, M. FitzGibbon, L. Deng, M. Royzen, J. Langridge \& D. Fabris, High-resolution ion mobility spectrometry-mass spectrometry of isomeric/isobaric ribonucleotide variants, Journal of Mass Spectrometry, 2020, 55, e4465.

281. D. Ropartz, M. Fanuel, J. Ujma, M. Palmer, K. Giles \& H. Rogniaux, Structure Determination of Large Isomeric Oligosaccharides of Natural Origin through Multipass and Multistage Cyclic Traveling-Wave Ion Mobility Mass Spectrometry, Analytical Chemistry, 2019, 91, 12030-12037.

282. M. E. Ridgeway, M. Lubeck, J. Jordens, M. Mann \& M. A. Park, Trapped ion mobility spectrometry: A short review, International journal of mass spectrometry, 2018, 425, 22-35.

283. F. Meier, A.-D. Brunner, S. Koch, H. Koch, M. Lubeck, M. Krause, N. Goedecke, J. Decker, T. Kosinski, M. A. Park, N. Bache, O. Hoerning, J. Cox, O. Räther \& M. Mann, Online Parallel Accumulation-Serial Fragmentation (PASEF) with a Novel Trapped lon Mobility Mass Spectrometer, Molecular \& Cellular Proteomics, 2018, 17, 2534-2545.

284. E. R. Schenk, M. E. Ridgeway, M. A. Park, F. Leng \& F. Fernandez-Lima, Isomerization kinetics of AT hook decapeptide solution structures, Analytical Chemistry, 2014, 86, 1210-1214.

285. B. M. Zietek, Y. Mengerink, J. Jordens, G. W. Somsen, J. Kool \& M. Honing, Adduct-ion formation in trapped ion mobility spectrometry as a potential tool for studying molecular structures and conformations, International Journal for Ion Mobility Spectrometry, 2018, 21, 19-32.

286. P. Benigni, C. J. Thompson, M. E. Ridgeway, M. A. Park \& F. Fernandez-Lima, Targeted highresolution ion mobility separation coupled to ultrahigh-resolution mass spectrometry of endocrine disruptors in complex mixtures, Analytical Chemistry, 2015, 87, 4321-4325. 
A. McKenzie-Coe, J. D. DeBord, M. Ridgeway, M. Park, G. Eiceman \& F. Fernandez-Lima, Lifetimes and stabilities of familiar explosive molecular adduct complexes during ion mobility measurements, The Analyst, 2015, 140, 5692-5699.

288. A. S. A, A. A. G \& D. S. R, Pushing the Frontier of High-Definition lon Mobility Spectrometry Using FAIMS, Mass Spectrometry (Tokyo), 2013, 2, S0011.

289. A. J. Creese \& H. J. Cooper, Separation of cis and trans Isomers of Polyproline by FAIMS Mass Spectrometry, Journal of the American Society for Mass Spectrometry, 2016, 27, 2071-2074.

290. A. A. Shvartsburg \& R. D. Smith, Protein analyses using differential ion mobility microchips with mass spectrometry, Analytical Chemistry, 2012, 84, 7297-7300.

291. A. A. Shvartsburg, Y. Zheng, R. D. Smith \& N. L. Kelleher, Separation of variant methylated histone tails by differential ion mobility, Analytical Chemistry, 2012, 84, 6317-6320.

292. K. E. Swearingen \& R. L. Moritz, High-field asymmetric waveform ion mobility spectrometry for mass spectrometry-based proteomics, Expert review of proteomics, 2012, 9, 505-517.

293. Y. Xuan, A. J. Creese, J. A. Horner \& H. J. Cooper, High-field asymmetric waveform ion mobility spectrometry (FAIMS) coupled with high-resolution electron transfer dissociation mass spectrometry for the analysis of isobaric phosphopeptides, Rapid Communications in Mass Spectrometry, 2009, 23, 1963-1969.

294. M. J. Kailemia, M. Park, D. A. Kaplan, A. Venot, G. J. Boons, L. Li, R. J. Linhardt \& I. J. Amster, High-field asymmetric-waveform ion mobility spectrometry and electron detachment dissociation of isobaric mixtures of glycosaminoglycans, Journal of the American Society for Mass Spectrometry, 2014, 25, 258-268.

295. R. L. Griffiths, A. Dexter, A. J. Creese \& H. J. Cooper, Liquid extraction surface analysis field asymmetric waveform ion mobility spectrometry mass spectrometry for the analysis of dried blood spots, The Analyst, 2015, 140, 6879-6885.

296. A. P. Bowman, R. R. Abzalimov \& A. A. Shvartsburg, Broad Separation of Isomeric Lipids by HighResolution Differential Ion Mobility Spectrometry with Tandem Mass Spectrometry, Journal of the American Society for Mass Spectrometry, 2017, 28, 1552-1561.

297. J. Kapron, J. Wu, T. Mauriala, P. Clark, R. W. Purves \& K. P. Bateman, Simultaneous analysis of prostanoids using liquid chromatography/high-field asymmetric waveform ion mobility spectrometry/tandem mass spectrometry, Rapid Communications in Mass Spectrometry, 2006, 20, 1504-1510.

298. M. Girod, E. Moyano, D. I. Campbell \& R. G. Cooks, Accelerated bimolecular reactions in microdroplets studied by desorption electrospray ionization mass spectrometry, Chemical Science, 2011, 2, 501-510.

299. D. F. Cobice, C. L. Mackay, R. J. A. Goodwin, A. McBride, P. R. Langridge-Smith, S. P. Webster, B. R. Walker \& R. Andrew, Mass Spectrometry Imaging for Dissecting Steroid Intracrinology within Target Tissues, Analytical Chemistry, 2013, 85, 11576-11584.

300. W. J. Griffiths, J. Abdel-Khalik, P. J. Crick, E. Yutuc \& Y. Wang, New methods for analysis of oxysterols and related compounds by LC-MS, The Journal of Steroid Biochemistry and Molecular Biology, 2016, 162, 4-26.

301. S. A. Shaffer, D. C. Prior, G. A. Anderson, H. R. Udseth \& R. D. Smith, An Ion Funnel Interface for Improved lon Focusing and Sensitivity Using Electrospray lonization Mass Spectrometry, Analytical Chemistry, 1998, 70, 4111-4119.

302. B.-L. Qi, P. Liu, Q.-Y. Wang, W.-J. Cai, B.-F. Yuan \& Y.-Q. Feng, Derivatization for liquid chromatography-mass spectrometry, TrAC Trends in Analytical Chemistry, 2014, 59, 121-132.

303. A. M. Gaddis, R. E. X. Ellis \& G. T. Currie, Girard T Reagent for Carbonyls, Nature, 1961, 191, 1391-1392.

304. A. S. Stachissini \& L. Do Amaral, Kinetics and mechanism of benzaldehyde Girard T hydrazone formation, Journal of Organic Chemistry, 1991, 56, 1419-1424.

305. O. H. Wheeler, The Girard reagents, Journal of Chemical Education, 1968, 45, 435.

306. M. J. Kimzey, H. N. Yassine, B. M. Riepel, G. Tsaprailis, T. J. Monks \& S. S. Lau, New Site(s) of Methylglyoxal-Modified Human Serum Albumin, Identified by Multiple Reaction Monitoring, Alter Warfarin Binding and Prostaglandin Metabolism, Chemico-biological interactions, 2011, 192, 122128.

307. J. Yang, C. E. Petersen, C. E. Ha \& N. V. Bhagavan, Structural insights into human serum albuminmediated prostaglandin catalysis, Protein Science, 2002, 11, 538-545.

308. S. Brombacher, S. J. Owen \& D. A. Volmer, Automated coupling of capillary-HPLC to matrixassisted laser desorption/ionization mass spectrometry for the analysis of small molecules utilizing a reactive matrix, Analytical and Bioanalytical Chemistry, 2003, 376, 773-779. 
309. D. F. Cobice, D. E. W. Livingstone, C. L. Mackay, R. J. A. Goodwin, L. B. Smith, B. R. Walker \& R. Andrew, Spatial Localization and Quantitation of Androgens in Mouse Testis by Mass Spectrometry Imaging, Analytical Chemistry, 2016, 88, 10362-10367.

310. W. J. Griffiths, S. Liu, G. Alvelius \& J. Sjövall, Derivatisation for the characterisation of neutral oxosteroids by electrospray and matrix-assisted laser desorption/ionisation tandem mass spectrometry: the Girard P derivative, Rapid Communications in Mass Spectrometry, 2003, 17, 924-935.

311. C. Eldrid, J. Ujma, S. Kalfas, N. Tomczyk, K. Giles, M. Morris \& K. Thalassinos, Gas Phase Stability of Protein lons in a Cyclic lon Mobility Spectrometry Traveling Wave Device, Analytical Chemistry, 2019, 91, 7554-7561.

312. K. Jeanne Dit Fouque, A. Garabedian, J. Porter, M. Baird, X. Pang, T. D. Williams, L. Li, A Shvartsburg \& F. Fernandez-Lima, Fast and Effective Ion Mobility-Mass Spectrometry Separation of d-Amino-Acid-Containing Peptides, Analytical Chemistry, 2017, 89, 11787-11794.

313. J. M. Wiseman, D. R. Ifa, Y. Zhu, C. B. Kissinger, N. E. Manicke, P. T. Kissinger \& R. G. Cooks, Desorption electrospray ionization mass spectrometry: Imaging drugs and metabolites in tissues, Proceedings of the National Academy of Sciences, 2008, 105, 18120-18125.

314. T. Vallianatou, N. Strittmatter, A. Nilsson, M. Shariatgorji, G. Hamm, M. Pereira, P. Kallback, P. Svenningsson, M. Karlgren, R. J. A. Goodwin \& P. E. Andren, A mass spectrometry imaging approach for investigating how drug-drug interactions influence drug blood-brain barrier permeability, Neurolmage, 2018, 172, 808-816.

315. M. Sans, C. L. Feider \& L. S. Eberlin, Advances in mass spectrometry imaging coupled to ion mobility spectrometry for enhanced imaging of biological tissues, Current opinion in chemical biology, 2018, 42, 138-146.

316. D. M. Anderson, Z. Ablonczy, Y. Koutalos, J. Spraggins, R. K. Crouch, R. M. Caprioli \& K. L. Schey, High resolution MALDI imaging mass spectrometry of retinal tissue lipids, Journal of the American Society for Mass Spectrometry, 2014, 25, 1394-1403.

317. A. Kiss, D. F. Smith, B. R. Reschke, M. J. Powell \& R. M. Heeren, Top-down mass spectrometry imaging of intact proteins by laser ablation ESI FT-ICR MS, Proteomics, 2014, 14, 1283-1289.

318. H. Takeda, Y. Izumi, M. Takahashi, T. Paxton, S. Tamura, T. Koike, Y. Yu, N. Kato, K. Nagase, M. Shiomi \& T. Bamba, Widely-targeted quantitative lipidomics methodology by supercritical fluid chromatography coupled with fast-scanning triple quadrupole mass spectrometry, Journal of Lipid Research, 2018, DOI: 10.1194/jlr.D083014.

319. D. Schwudke, J. Oegema, L. Burton, E. Entchev, J. T. Hannich, C. S. Ejsing, T. Kurzchalia \& A. Shevchenko, Lipid Profiling by Multiple Precursor and Neutral Loss Scanning Driven by the DataDependent Acquisition, Analytical Chemistry, 2006, 78, 585-595.

320. G. Hopfgartner, E. Varesio \& M. Stoeckli, Matrix-assisted laser desorption/ionization mass spectrometric imaging of complete rat sections using a triple quadrupole linear ion trap, Rapid Communications in Mass Spectrometry, 2009, 23, 733-736.

321. S. M. Boudon, G. Morandi, B. Prideaux, D. Staab, U. Junker, A. Odermatt, M. Stoeckli \& D. Bauer, Evaluation of Sparfloxacin Distribution by Mass Spectrometry Imaging in a Phototoxicity Model, Journal of the American Society for Mass Spectrometry, 2014, 25, 1803-1809.

322. P. Michael, B. M. R., S. A. E. \& K. Thomas, Segmental hair analysis for differentiation of tilidine intake from external contamination using LC-ESI-MS/MS and MALDI-MS/MS imaging, Drug testing and analysis, 2015, 7, 143-149.

323. T. Porta, C. Grivet, T. Kraemer, E. Varesio \& G. Hopfgartner, Single Hair Cocaine Consumption Monitoring by Mass Spectrometric Imaging, Analytical Chemistry, 2011, 83, 4266-4272.

324. F. F. Hsu \& J. Turk, Electrospray ionization with low-energy collisionally activated dissociation tandem mass spectrometry of glycerophospholipids: mechanisms of fragmentation and structural characterization, Journal of Chromatography B, 2009, 877, 2673-2695.

325. F. F. Hsu \& J. Turk, Studies on phosphatidylserine by tandem quadrupole and multiple stage quadrupole ion-trap mass spectrometry with electrospray ionization: structural characterization and the fragmentation processes, Journal of the American Society for Mass Spectrometry, 2005, $16,1510-1522$

326. J. Vessman, Selectivity or specificity? Validation of analytical methods from the perspective of an analytical chemist in the pharmaceutical industry, Journal of pharmaceutical and biomedical analysis, 1996, 14, 867-869.

327. A. R. Korte \& Y. J. Lee, Multiplex Mass Spectrometric Imaging with Polarity Switching for Concurrent Acquisition of Positive and Negative Ion Images, Journal of the American Society for Mass Spectrometry, 2013, 24, 949-955. 
M. Nazari \& D. C. Muddiman, Polarity switching mass spectrometry imaging of healthy and cancerous hen ovarian tissue sections by infrared matrix-assisted laser desorption electrospray ionization (IR-MALDESI), The Analyst, 2016, 141, 595-605.

329. M. S. Benedetti, R. Whomsley, I. Poggesi, W. Cawello, F. X. Mathy, M. L. Delporte, P. Papeleu \& J. B. Watelet, Drug metabolism and pharmacokinetics, Drug metabolism reviews, 2009, 41, 344390.

330. I. Rzagalinski \& D. A. Volmer, Quantification of low molecular weight compounds by MALDI imaging mass spectrometry - A tutorial review, Biochimica et Biophysica Acta - Proteins and Proteomics, 2017, 1865, 726-739.

331. K. Fuchs, A. Kiss, P. E. Bize, R. Duran, A. Denys, G. Hopfgartner, G. Borchard \& O. Jordan, Mapping of drug distribution in the rabbit liver tumor model by complementary fluorescence and mass spectrometry imaging, Journal of Controlled Release, 2018, 269, 128-135.

332. J. G. Swales, A. Dexter, G. Hamm, A. Nilsson, N. Strittmatter, F. Michopoulos, C. Hardy, P. Morentin-Gutierrez, M. Mellor, P. E. Andren, M. R. Clench, J. Bunch, S. E. Critchlow \& R. J. A. Goodwin, Quantitation of Endogenous Metabolites in Mouse Tumors Using Mass-Spectrometry Imaging, Analytical Chemistry, 2018, 90, 6051-6058.

333. L. Jadoul, N. Smargiasso, F. Pamelard, D. Alberts, A. Noel, E. De Pauw \& R. Longuespee, An Improved Molecular Histology Method for Ion Suppression Monitoring and Quantification of Phosphatidyl Cholines During MALDI MSI Lipidomics Analyses, Omics : a journal of integrative biology, 2016, 20, 110-121.

334. H. T. Hansen \& C. Janfelt, Aspects of Quantitation in Mass Spectrometry Imaging Investigated on Cryo-Sections of Spiked Tissue Homogenates, Analytical Chemistry, 2016, 88, 11513-11520.

335. J. A. Barry, R. Ait-Belkacem, W. M. Hardesty, L. Benakli, C. Andonian, H. Licea-Perez, J. Stauber \& S. Castellino, Multicenter Validation Study of Quantitative Imaging Mass Spectrometry, Analytical Chemistry, 2019, DOI: 10.1021/acs.analchem.9b01016.

336. J. A. Barry, M. R. Groseclose \& S. Castellino, Quantification and assessment of detection capability in imaging mass spectrometry using a revised mimetic tissue model, Bioanalysis, 2019, 11, 10991116.

337. R. J. A. Goodwin, Sample preparation for mass spectrometry imaging: Small mistakes can lead to big consequences, Journal of Proteomics, 2012, 75, 4893-4911.

338. R. J. Goodwin, P. Scullion, L. Macintyre, D. G. Watson \& A. R. Pitt, Use of a solvent-free dry matrix coating for quantitative matrix-assisted laser desorption ionization imaging of 4-bromophenyl-1,4diazabicyclo(3.2.2)nonane-4-carboxylate in rat brain and quantitative analysis of the drug from laser microdissected tissue regions, Analytical Chemistry, 2010, 82, 3868-3873.

339. P. Kovarik, C. Grivet, E. Bourgogne \& G. Hopfgartner, Method development aspects for the quantitation of pharmaceutical compounds in human plasma with a matrix-assisted laser desorption/ionization source in the multiple reaction monitoring mode, Rapid Communications in Mass Spectrometry, 2007, 21, 911-919.

340. L. Lamont, G. B. Eijkel, E. A. Jones, B. Flinders, S. R. Ellis, T. Porta, R. M. A. Heeren \& R. J. Vreeken, Targeted Drug and Metabolite Imaging: Desorption Electrospray lonization combined with Triple Quadrupole Mass Spectrometry, Analytical Chemistry, 2018, DOI: 10.1021/acs.analchem.8b03857.

341. B. Magnusson \& U. Ornemark, Eurachem Guide: The Fitness for Purpose of Analytical Methods A Laboratory Guide to Method Validation and Related Topics, (2nd ed. 2014), ISBN 978-9187461-59-0, Available from http://www.eurachem.org.

342. X. Liang, S. Ubhayakar, B. M. Liederer, B. Dean, A. Ran-Ran Qin, S. Shahidi-Latham \& Y. Deng, Evaluation of homogenization techniques for the preparation of mouse tissue samples to support drug discovery, Bioanalysis, 2011, 3, 1923-1933.

343. E. L. Gill, R. A. Yost, V. Vedam-Mai \& T. J. Garrett, Precast Gelatin-Based Molds for Tissue Embedding Compatible with Mass Spectrometry Imaging, Analytical Chemistry, 2017, 89, 576580.

344. A. F. Altelaar, J. van Minnen, C. R. Jimenez, R. M. Heeren \& S. R. Piersma, Direct molecular imaging of Lymnaea stagnalis nervous tissue at subcellular spatial resolution by mass spectrometry, Analytical Chemistry, 2005, 77, 735-741.

345. L. L. Jessome \& D. A. Volmer, Ion Suppression: A Major Concern in Mass Spectrometry, LCGC North America, 2006, 24, 498-510.

346. European, Medicine \& Agency, Guideline on Bioanalytical Method Validation, 2009.

347. A. Shrivastava \& V. Gupta, Methods for the determination of limit of detection and limit of quantitation of the analytical methods, Chronicles of Young Scientists, 2011, 2, 21-25. 
348. Y. Ibrahim, K. Tang, A. V. Tolmachev, A. A. Shvartsburg \& R. D. Smith, Improving mass spectrometer sensitivity using a high-pressure electrodynamic ion funnel interface, Journal of the American Society for Mass Spectrometry, 2006, 17, 1299-1305.

349. B. Flinders, L. R. S. Huizing, M. van Heerden, F. Cuyckens, U. P. Neumann, L. J. W. van der Laan, S. W. M. Olde Damink, R. M. A. Heeren, F. G. Schaap \& R. J. Vreeken, Cross-Species Molecular Imaging of Bile Salts and Lipids in Liver: Identification of Molecular Structural Markers in Health and Disease, Analytical Chemistry, 2018, DOI: 10.1021/acs.analchem.8b01378.

350. J. F. Cahill, V. Kertesz, T. M. Weiskittel, M. Vavrek, C. Freddo \& G. J. Van Berkel, Online, Absolute Quantitation of Propranolol from Spatially Distinct 20- and 40-mum Dissections of Brain, Liver, and Kidney Thin Tissue Sections by Laser Microdissection-Liquid Vortex Capture-Mass Spectrometry, Analytical Chemistry, 2016, 88, 6026-6034.

351. M. Oya, H. Suzuki, A. R. J. Anas, K. Oishi, K. Ono, S. Yamaguchi, M. Eguchi \& M. Sawada, LCMS/MS imaging with thermal film-based laser microdissection, Analytical and Bioanalytical Chemistry, 2017, DOI: 10.1007/s00216-017-0739-2.

352. R. L. Griffiths, K. I. Kocurek \& H. J. Cooper, Ambient surface mass spectrometry-ion mobility spectrometry of intact proteins, Current opinion in chemical biology, 2017, 42, 67-75. 


\subsection{List of abbreviations}

\begin{tabular}{|c|c|}
\hline$A C$ & Alternating current \\
\hline ACN & Acetonitrile \\
\hline$A D C$ & Analog-to-digital converters \\
\hline ADME & Absorption, distribution, metabolism, and excretion \\
\hline CE & Collision energy \\
\hline CID & Collision-induced dissociation \\
\hline cIMS & Cyclic ion mobility spectrometry \\
\hline $\operatorname{cox}$ & Cyclooxygenase \\
\hline CRM & Charge residue model \\
\hline $\mathrm{CV}$ & Compensation voltage \\
\hline CZE & Capillary zone electrophoresis \\
\hline $\mathrm{Da}$ & Dalton \\
\hline DC & Direct current \\
\hline DDA & Data-dependent acquisition \\
\hline DESI & Desorption electrospray ionization \\
\hline DTIMS & Drift-time ion mobility spectrometry \\
\hline EMA & European Medicine Agency \\
\hline ESI & Electrospray ionization \\
\hline $\mathrm{EtOH}$ & Ethanol \\
\hline FA & Formic acid \\
\hline FAIMS & Field asymmetric ion mobility spectrometry \\
\hline FT-ICR & Fourier Transform Ion Cyclotron Resonance mass analyzer \\
\hline GC & Gas chromatography \\
\hline GT & Girard's reagent T \\
\hline$H \& E$ & Hematoxylin and eosin \\
\hline $\mathrm{H} 2 \mathrm{O}$ & Water \\
\hline HPLC & High performance liquid chromatrography \\
\hline HR & High resolution \\
\hline IEM & Ion evaporation model \\
\hline IMS & Ion mobility spectrometry \\
\hline IS & Internal standard \\
\hline IV & Intravenous \\
\hline LA/LCSA & Laser ablation liquid capture surface analysis \\
\hline LAESI & Laser ablation electrospray ionization \\
\hline LC & Liquid chromatography \\
\hline LCM & Laser capture microdissection \\
\hline LESA & Liquid extraction surface analysis \\
\hline LeuEnk & Leucine enkephalin \\
\hline LIT & Linear ion trap mass analyzer \\
\hline LLOQ & Lower limit of quantification \\
\hline LMJ/SSP & Liquid microjunction surface sampling probe \\
\hline LOD & Limit of detection \\
\hline$m / z$ & mass-to-charge ratio \\
\hline MALDI & Matrix-assisted laser desorption ionization \\
\hline $\mathrm{MeOH}$ & Methanol \\
\hline MRM & Multiple reaction monitoring \\
\hline
\end{tabular}




\begin{tabular}{|c|c|}
\hline MS & Mass spectrometry \\
\hline MS/MS & Tandem mass spectrometry \\
\hline MSE/DIA & Data-independent acquisition \\
\hline MSI & Mass spectrometry imaging \\
\hline nano-DESI & Nanospray desorption electrospray ionization \\
\hline NL & Neutral loss scan \\
\hline NMR & Nuclear magnetic resonance \\
\hline PC & Phosphatidylcholine \\
\hline PD & Pharmacodynamics \\
\hline PE & Phosphatidylethanolamine \\
\hline $\mathrm{PGD}_{2}$ & Prostaglandin $\mathrm{D}_{2}$ \\
\hline $\mathrm{PGE}_{2}$ & Prostaglandin $\mathrm{E}_{2}$ \\
\hline $\mathrm{PGF}_{2 a}$ & Prostaglandin $\mathrm{F}_{2 a}$ \\
\hline PGHS & Prostaglandin $\mathrm{G} / \mathrm{H}$ synthases \\
\hline $\mathrm{PGI}_{2}$ & Prostaglandin $I_{2}$ \\
\hline PK & Pharmacokinetics \\
\hline POMC & Pro-opiomelanocortin \\
\hline PREC & Precursor ion scan \\
\hline PROD & Product ion scan \\
\hline PS & Phosphatidylserine \\
\hline PTM & Post-translational modifications \\
\hline QC & Quality control \\
\hline Q-MSI & Quantitative mass spectrometry imaging \\
\hline QqQ & Triple quadrupole mass spectrometer \\
\hline Q-TOF & Quadrupole time-of-flight mass spectrometer \\
\hline Q-WBA & Quantitative whole body autoradiography \\
\hline RF & Radio frequency \\
\hline $\mathrm{ROI}$ & Region of interest \\
\hline RSD & Relative standard deviation \\
\hline SD & Standard deviation \\
\hline SIM & Selected ion monitoring \\
\hline SIMS & Secondary ion mass spectrometry \\
\hline SM & Sphingomyelin \\
\hline SRM & Selected reaction monitoring \\
\hline SWATH & Sequential windowed acquisition of all theoretical fragment ion mass spectra \\
\hline TEC & Tissue extinction coefficient \\
\hline TIMS & Trapped ion mobility spectrometry \\
\hline TOF & Time-of-flight mass analyzer \\
\hline $\mathrm{TQ}$ & Tandem quadrupole mass spectrometer \\
\hline TWIMS & Travelling wave ion mobility spectrometry \\
\hline $\mathrm{TxA}_{2}$ & Thromboxane $A_{2}$ \\
\hline$U(H) P L C$ & Ultra (high) performance liquid chromatography \\
\hline ULOQ & Upper limit of quantification \\
\hline WBA & Whole body autoradiography \\
\hline $\mathrm{XIC}$ & Extracted ion chromatogram \\
\hline
\end{tabular}




\subsection{List of publications}

\section{This thesis is based on the following publications}

Integration of Ion Mobility MS ${ }^{\mathrm{E}}$ after Fully Automated, Online, High-Resolution Liquid Extraction Surface Analysis Micro-Liquid Chromatography

Lieke Lamont, Mark Baumert, Nina Ogrinc, Mark Allen, Rob J. Vreeken, Ron M.A. Heeren and Tiffany Porta

Analytical Chemistry 201789 (20) 11143-11150

High Resolution Ion Mobility Separation Mass Spectrometry for Isomeric Separation of Prostanoids after Derivatization

Lieke Lamont, Andrew P. Bowman, Darya Hadavi, Dale Cooper-Shepherd, Martin Palmer, Bryn Flinders, Jan Jordens, Ynze Mengerink, Maarten Honing, James Langridge, Tiffany Porta Siegel, Rob J. Vreeken and Ron M. A. Heeren

Manuscript in preparation

Targeted Drug and metabolite imaging: Desorption Electrospray Ionization Combined with Triple Quadrupole Mass Spectrometry

Lieke Lamont, Gert B. Eijkel, Emrys A. Jones, Bryn Flinders, Shane R. Ellis, Tiffany Porta Siegel, Ron M.A. Heeren and Rob J. Vreeken

Analytical Chemistry 201890 (22), 13235-1323

Quantitative Mass Spectrometry Imaging of Drugs and Metabolites: a Multiplatform Comparison

Lieke Lamont, Darya Hadavi, Brent Viehmann, Bryn Flinders, Ron M. A. Heeren, Rob J. Vreeken, and Tiffany Porta Siegel

Manuscript submitted for publication

\section{Other publications}

Derivatization of Carboxylic Acids with 4-APEBA for Detection by Positive-ion LCESI-MS (/MS) Applied for the Analysis of Prostanoids and NSAID in Urine Ansgar Kretschmer, Martin Giera, Maikel Wijtmans, Lieke de Vries, Henk Lingeman, Hubertus Irth and Wilfried M.A. Niessen

Journal of Chromatography B 2011879 (17-18) 1393-1401 
Identification of inorganic pigments used in porcelain cards based on fusing Raman and X-ray fluorescence (XRF) data

Annelien Deneckere, Lieke de Vries, Bart Vekemans, Lien Van de Voorde, Freek Ariese, Laszlo Vincze, Luc Moens and Peter Vandenabeele

Applied Spectroscopy 201165 (11) 1281-1290

Ion Mobility Spectrometry Combined with Multivariate Statistical Analysis: Revealing the Effects of a Drug Candidate for Alzheimer's Disease on A $\boldsymbol{\beta} 1-40$ Peptide Early Assembly

Serena Lazzaro, Nina Ogrinc, Lieke Lamont, Graziella Vecchio, Giuseppe Pappalardo and Ron M.A. Heeren

Analytical and Bioanalytical Chemistry 2019411 (24) 6353-6363 


\subsection{Acknowledgements}

After 5 years, this PhD is coming to an end and I would like to express my sincere gratitude to everyone who contributed to the completion of this thesis.

Prof. dr. Ron M.A. Heeren, Beste Ron, ontzettend bedankt dat ik dit promotieonderzoek bij jou, bij M4I, mocht uitvoeren en dat ik kennis heb mogen maken met MSI. Wat een fantastisch werkveld! Bedankt voor alles dat ik van jou heb mogen leren en bedankt voor jouw aanstekelijke enthousiasme. Het is een onvergetelijke tijd geweest!

Dr. Rob J. Vreeken, Beste Rob, wat een kleine wereld. Nog geen 2 jaar nadat we elkaar leerden kennen in Leiden, samen aan mijn promotietraject beginnen. Ontzettend bedankt voor al je kennis, je steun en support tijdens mijn promotietraject. Bedankt voor alle leerzame vrijdagochtenden!

Dr. Tiffany Porta Siegel, Dear Tiff, I am so grateful to have had you on my team of supervisors. Thank you so much for your support and always being available for my questions. Thank you for your knowledge and the fun time we had in and outside work. It has been an amazing journey!

I would like to thank everyone I met during my time at M4I: Benjamin, Berta, Shane, Maarten, Nina, Keely, Marty, Joel, Marta, Bea, Peter, Pmax, Fred, Florian, Maxime, Michele, Naomi, Klara, Andrew, Britt, Stephanie, Sylvia, Jianhua, Martha, Fanny, Arnoud, Christel, Daan, Brent, Jonah, Ronny, Livia, and Helen. A special thank you to Bryn and Lennart, my Janssen brothers, for all the discussions on Friday morning during the Janssen meetings. Frans, thank you for your support in and outside the lab. Gert, thank you for your amazing data analysis support. Hang, Jo, Nino, and Abril for the nice dinners and support. Kees, Jos, and Darya for all the fun in the office. My running, cycling, and bootcamp buddies Pieter, Anne, and Brenda, thank you for the unforgettable time in Maastricht! Anne and Brenda, thank you for being my paranymphs during the defense. I would also like to thank my colleagues at the Nanoscopy department, the Surgery department, and IDEE.

I am very grateful to have had the opportunity to collaborate with researchers from all over the world who contributed greatly to this thesis throughout the years: Filip Cuyckens (Thank you for approaching me for this PhD position!), Ronald de Vries, Marjolein van Heerden, and Farid Jahouh from Janssen R\&D; Emmanuelle Claude, Emrys Jones, Mark Towers, Martin Palmer, Dale Cooper-Shepherd, James Langridge, and Aurelien Boland from Waters Corporation; Daniel Eikel, Mark Allen and Mark Baumert 
from Advion; Jan Jordens and Ynze Mengerink from DSM; and Alexandre Shvartsburg from Wichita State University.

Analytical chemistry in the Netherlands is a very small world and I am very grateful to those that have educated me throughout the years prior to my PhD: Ab Buijtenhuijs (Akzo Nobel), Dr. Henk Lingeman (VU University), Dr. Freek Ariese (VU University), Prof. dr. Peter Vandenabeele (UGent), Dr. Martijn Schenning (Crucell), Dr. Ewoud van Tricht (Crucell), Prof. dr. Thomas Hankemeier (Leiden University), and Dr. Amy Harms (Leiden University).

I would like to thank Prof. dr. Thomas Hankemeier, Dr. Amy Harms, and my new colleagues at the LACDR for the warm welcome (back) in Leiden. In particular, Alida Kindt for your help with the introduction of this thesis.

Ik wil mijn familie en vrienden bedanken. Mijn ouders Bé \& Janny, Femke \& Koen, Stijn, Thom \& Christel, bedankt voor jullie onvoorwaardelijke steun! My family-in-law in Scotland for their support. Lisa, Romina en Isabelle, bedankt voor het luisterende oor tijdens onze etentjes en festivals. Romina voor de fantastische cover! Marian, bedankt dat je Maastricht mijn tweede thuis hebt gemaakt.

My dearest Chris, love of my life, words cannot express my gratitude to you but I am still going to try. From day one, you understood what it takes to complete a PhD. Thank you so much for all your support and for all the Friday nights when I came home and needed to vent my workweek. I am so incredibly proud of you and grateful to have you by my side. Liefste Aria, het laatste jaar van mijn promotietraject was het allermooiste jaar met jou bij ons. Ik hoop dat ook jij in de toekomst je dromen zult nastreven. Bedankt dat ik jouw mama mag zijn! 


\subsection{Curriculum Vitae}

Lieke Lamont-de Vries was born on September 28 ${ }^{\text {th }}$, 1988 in Doetinchem, The Netherlands. After completing secondary school at the St-Ludgercollege in 2005, she started her Analytical Chemistry education at the Hogeschool van Arnhem en Nijmegen (HAN) in Nijmegen. This education has brought her internships at Akzo Nobel (Arnhem) and at the VU University (Amsterdam). Throughout these internships, she gained experience with liquid chromatography, capillary electrophoresis, fluorescence spectroscopy, and mass spectrometry. With increased interest in Analytical Chemistry, she continued her Master's degree at VU University Amsterdam in 2009. Her final internship was executed at the Ghent University using Raman spectroscopy and X-ray fluorescence to identify pigments on $19^{\text {th }}$ century porcelain cards.

Lieke began her professional career in $\mathbf{2 0 1 2}$ working as technician at Crucell (now Janssen Pharmaceutica) and at the Biomedical Metabolomics Facility Leiden, Leiden University as a metabolomics research technician. In both positions, she extended her experience in liquid chromatography and mass spectrometry for protein and metabolite analysis. In December 2015, she started her PhD research described in this thesis under the supervision of Prof. dr. Ron M. A. Heeren, Dr. Rob J. Vreeken, and Dr. Tiffany Porta Siegel at the Maastricht MultiModal Molecular Imaging (M4I) institute in Maastricht. This research was performed in collaboration with Janssen Pharmaceutica and investigated enhanced separation in pharmaceutical MSI. Parts of this research were presented at national (NVMS) and international conferences (OurCon V, MSACL US, and IMSC). Lieke occupied the role as PhD representative for M4I in the Faculty PhD Committee and was involved in the organization of OurCon V in September 2017. In 2020, she started a new position as postdoctoral researcher at the LACDR in the group of Prof. dr. Thomas Hankemeier in Leiden. 






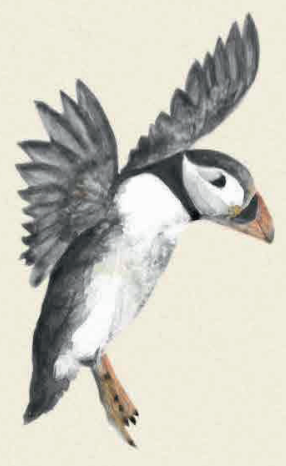

$?$

9
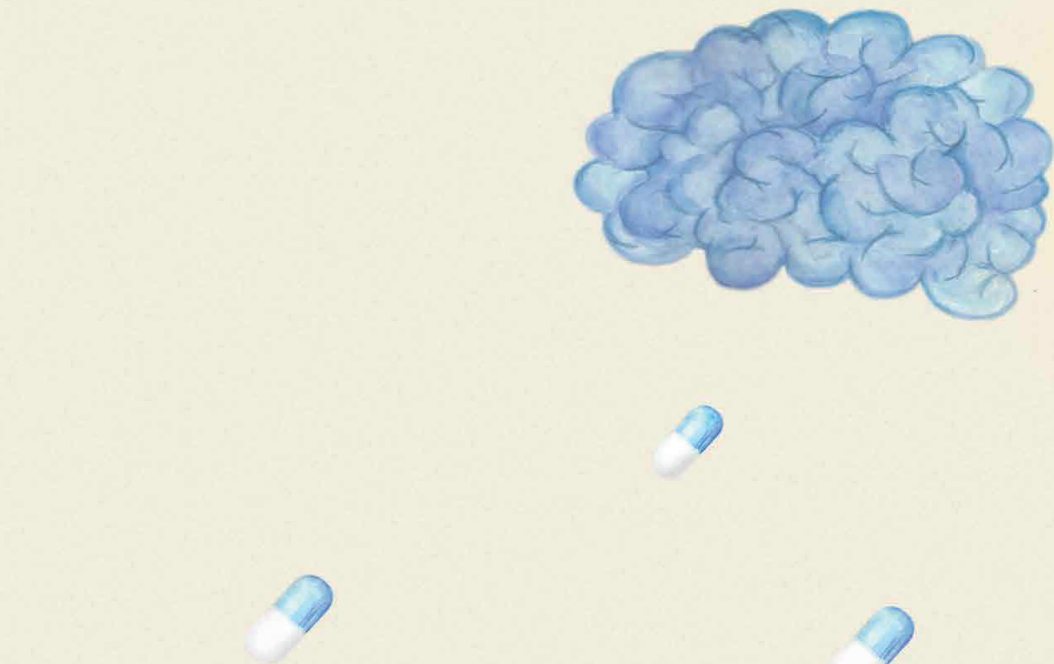

?

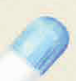

9

93

3
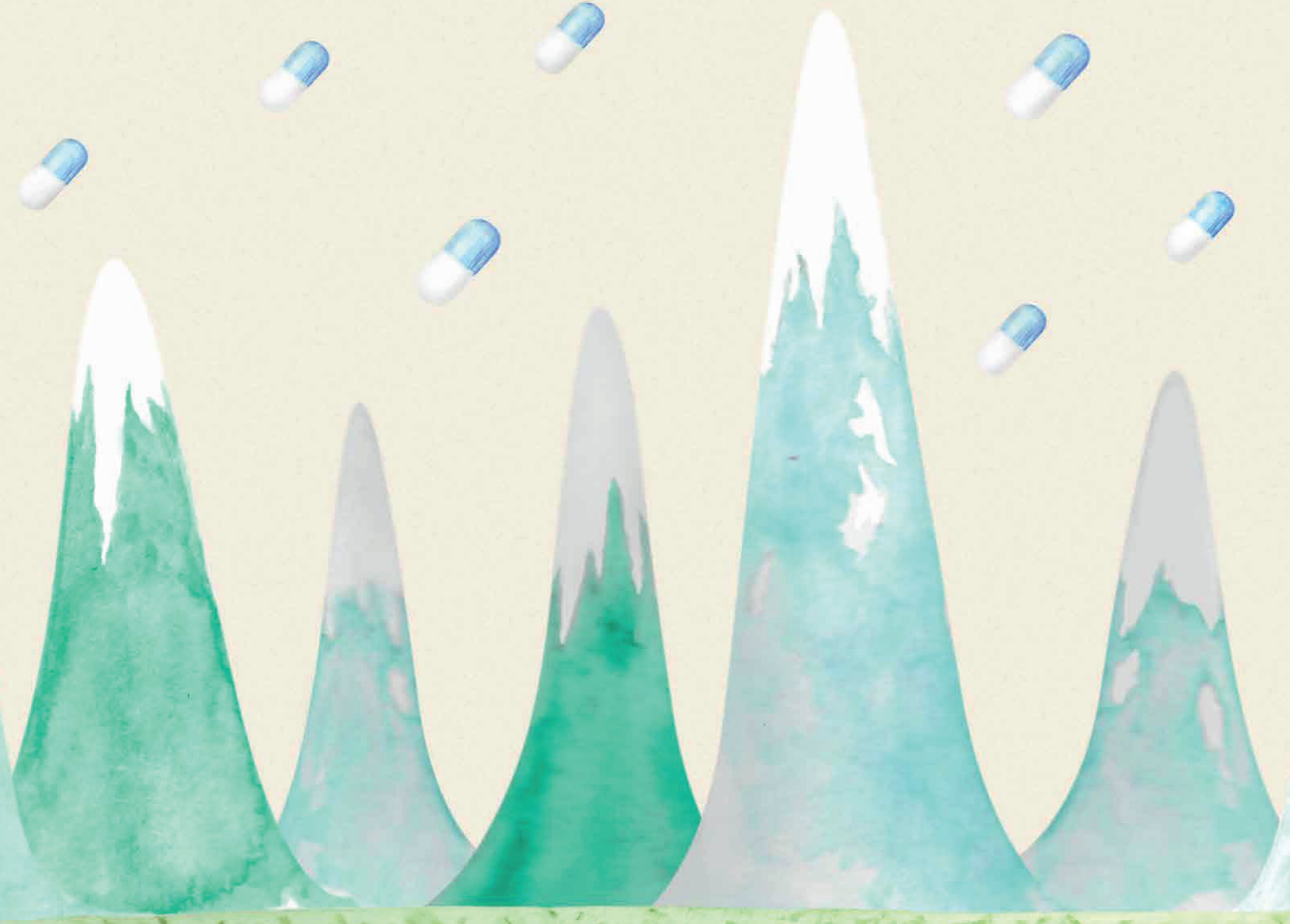

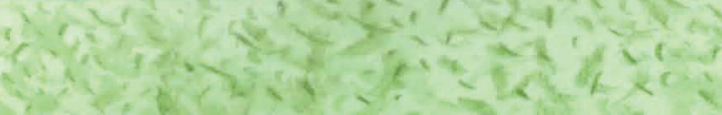

